\title{
Tactical plan recognition
}

Citation for published version (APA):

Mulder, F. (2005). Tactical plan recognition. [Doctoral Thesis, Maastricht University]. Universiteit Maastricht. https://doi.org/10.26481/dis.20050512fm

Document status and date:

Published: 01/01/2005

DOI:

10.26481/dis.20050512fm

Document Version:

Publisher's PDF, also known as Version of record

\section{Please check the document version of this publication:}

- A submitted manuscript is the version of the article upon submission and before peer-review. There can be important differences between the submitted version and the official published version of record.

People interested in the research are advised to contact the author for the final version of the publication, or visit the DOI to the publisher's website.

- The final author version and the galley proof are versions of the publication after peer review.

- The final published version features the final layout of the paper including the volume, issue and page numbers.

Link to publication

\footnotetext{
General rights rights.

- You may freely distribute the URL identifying the publication in the public portal. please follow below link for the End User Agreement:

www.umlib.nl/taverne-license

Take down policy

If you believe that this document breaches copyright please contact us at:

repository@maastrichtuniversity.nl

providing details and we will investigate your claim.
}

Copyright and moral rights for the publications made accessible in the public portal are retained by the authors and/or other copyright owners and it is a condition of accessing publications that users recognise and abide by the legal requirements associated with these

- Users may download and print one copy of any publication from the public portal for the purpose of private study or research.

- You may not further distribute the material or use it for any profit-making activity or commercial gain

If the publication is distributed under the terms of Article $25 \mathrm{fa}$ of the Dutch Copyright Act, indicated by the "Taverne" license above, 


\section{Tactical Plan Recognition}

\section{PROEFSCHRIFT}

ter verkrijging van de graad van doctor aan de Universiteit Maastricht, op gezag van de Rector Magnificus, Prof. mr. G. P. M. F. Mols volgens het besluit van het College van Decanen, in het openbaar te verdedigen op donderdag 12 mei 2005 om 12.00 uur

door 
Promotor:

Prof. dr. ir. K.L. Boon

Co-promotor:

Dr. P.J. Braspenning

Beoordelingscommissie:

Prof. dr. M.P.F. Berger

Prof. dr. D. Hammer (Universiteit Groningen, Technische Universiteit Eindhoven)

Dr. ir. M.A.W. Houtsma (Thales Nederland B.V.)

Prof. dr. A. Kolen

Prof. dr. ir. drs. O.J. Vrieze (voorzitter)

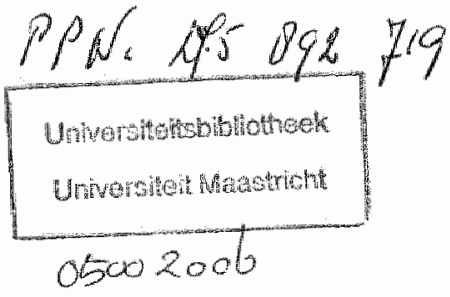




\title{
Tactical Plan Recognition
}

\author{
Frank Mulder
}

February 25, 2005 



\section{Preface}

In one of Arthur Conan Doyle's short stories, Sherlock Holmes remarkes: 'Once explained, everything becomes commonplace', when inspector Lestrade uttered 'I also knew that' after Holmes explained some observations of a murder scene. Of course, Lestrade conld not explain the observations himself and thought Holmes' explanation to be obvious, but this only after he heard it. Only when Holmes had given Lestrade an explanation of observations, Lestrade could understand the observations.

This short text intends to illustrate that observations of an unknown situation do not lead to understanding the situation, but rather hypotheses or an explanation does. The hypotheses can 'explain' the observations and, thus, lead to an understanding of the unknown situation.

The topic of this thesis is about observations of a particular type of worlds, that is: 'worlds' with unidentified objects and observations about these unidentified objects. The goal here, is to explain those uniclentified observations automatically by proposing one or more hypotheses about the world.

This thesis describes my research in the area of Command and Control systems. Command and Control systems assist in the management of operations of civil or military agencies, but they are mostly developed and applied in military applications (naval, army, air force applications). An important task of such a C\&C system is Tactical Plan Recognition.

My research was done under supervision of Peter Braspenning. The work on plan recognition logically followed my previous research in Generic Task Models of Peter Braspenning and myself in the period 1988-1993. It's subject was the design and implementation of reactive planning problems and its application to an Air Traffic Control problem: approach control. This research resulted in several publications at the Dutch National Artificial Intelligence Conferences, see Mulder and Braspenning [59, 60, 64]. For this a fairly large computer program was devised, consisting of various expert systems that co- 
operated through a distributed blackboard system.

Here the SPLICE distributed blackboard was used. SPLICE is a very elegant implementation of a distributed database using a Consumer-Producer paradigm for inter process communication. By means of SPLICE a transparent distributed database is created, which can be used as a distributed blackboard, see Mulder and Boasson [57, 58]. SPLICE has been developed by Maarten Boasson.

Also in the period 1990-1993, I was a member of the ARTIST-MUSICIAN project at Thales Nederland B.V., that focussed on new techniques for multiple target multiple sensor tracking. 'Tracking' is a type of computerized data processing that has as input position measurements of unidentified objects in the outside world. The output of the tracking function are tracks. Tracks give a compilation of the behavior of the measured object (an airplane, ship or vehicle). The measured objects are also called targets and the measurements are also called plots or observations. Two tasks of tracking are: first, to decide which plots originate from the same object and, second to estimate the trajectory of the target. The trajectory is defined by the target's of kinematic states as it varies in time.

In MUSICIAN we aimed at developing a completely new tracking system, malking a leap in technology from the more heuristic tracking algorithms used at that time. In the MUSICIAN project, the Multiple Hypothesis Tracking (MHT) algorithm from Donald Reid [74] was applied very successfully. During the MUSICIAN project, I also worked on the track correlation problem, see Mulder $[54,55]$. The goal here was: to decide, which newly initiated tracks could be a continuation of previously lost tracks. To solve this problem, it was necessary to consider the medium or longer term plan of a target being tracked. This contrary to the tracking problem itself, which was only concerned with short term kinematic states of an object being tracked. However, as the initiated-lost track application only considered kinematic data from the tracks, it is only suitable for a very limited set of scenarios. That is, the set of scenarios where no major change in direction and speed was executed by the target. For reasoning about long term plans of the target, it was necessary to apply more information about the target: its doctrines and its environment, than is provicled by the tracks.

After this project I visited a lecture about plan recognition in 1993 at the University of Brabant, describing recognition by an automated car driver of the driving plans of other drivers by observing them. A year later some 
literature on Tactical Plan Recognition of Azarewicz $[7,8]$ resulted in my first document on the subject and a second report on plan templates. The term tactical refers to medium term plans which will change the planning world medium term.

These documents introduced the research topic of Tactical Plan Recognition. They presented some insight in the structure of Tactical Plan Recognition by defining a Generic Task Model for it and here previous results from my reactive planning research could be applied very well. However, the documents failed to describe a correct definition of the hypotheses, and their uncertainty, of a Tactical Plan Recognition system. The main problem here is that unidentified observations are to be handled by the plan recognition system. Here, one does not know which input observations represent the same object in the outside world. Here, observations cannot be related, e.g. by their identification number, to previous observations.

Therefore, I proposed the following: do not state plan hypotheses that are directly supported by (necessarily identified) observations, but rather hypotheses about assignments of unidentified observations to hypothetical plans. An assignment relates each unidentified observation uniquely to particular hypothetical plans.

It is in some sense similar to the situation of multiple target, multiple sensor tracking in which the observations are also unidentified. When one states assignment hypotheses, Reid's Multiple Hypothesis Tracking (MHT) method can be adapted to our problem of Tactical Plan Recognition. Reid's formula for the likelihood of an assignment can be used to rank the assignment hypotheses. Not only assignments to previous plans are considered, but also the hypotheses that newly started plans are observed or a false observation is entered in the plan recognition system.

This proposal was first mentioned first in a paper for the IFIPS conference of 1998 [56]. The new MHT approach to Tactical Plan Recognition was first presented to the data and information fusion community in a paper for the Information Fusion Journal, co-authored with Frans Voorbraak [65, 66].

This MHT approach differs significantly from the mainstream of enemy plan recognition research in which plan recognizers only handle identified observations. In mainstream research on Tactical Plan Recognition, the plan recognizer cannot process unidentified observations directly. In mainstream research, first a sequence of unidentified observations are correlated with each other before doing plan recognition. The output of the correlator are then 
sets of observations that are expected to originate from the same object in the outside world. These identified observations are then input to the plan recognizer.

In this period, Edwin de Jong implemented a Multi Target Multi Tracking application in the purely functional language Haskell [43]. It was a full fledged Multiple Hypothesis Tracking application with Kalman filtering. It exemplified the great possibilities of Haskell. His program was my first introduction to Haskell. His work on this subject was applied in an international research project: the "TA10 data fusion project. Also, his organization of the Multiple Hypothesis Tracking was the basis of the MSDFLib sensor data fusion library, that will be discussed in the next paragraph. The plan recognizers, shown in this thesis, are also implemented in Haskell.

For some years after 1998 it was not possible to continue my research, due to my involvement data fusion project, that aimed at the development of a generic data fusion library: the MSDFLib, see Mulder et.al. and Driessen et.al. $[62,29]$. But in 2002 some work on the user interface and a Haskell implementation of plan recognition with identified observations could be done. This was done in the context of the Digital Battle Space research program of Thales Nederland B.V. In 2003 the research was continued, resulting in this thesis.

\section{Acknowledgements}

In an early stage, this research was a part of the MOSAIC project of Prof. Maarten Boasson of the University of Amsterdam. The follow up research was done in the context of the Digital Battle Space project at Thales Nederland B.V. of Harry Menninga of Thales Nederland B.V.

During all the years of worlk on this subject, I was encouraged and supported by Dr. Peter Braspenning of the group Communications Research and Semiotics of the University of Maastricht. As I have been working for a commercial firm, I you are forced to write rather short technical reports or, even worse, power point presentations. Writing a book is quite a different craft, and the experience and critical eye of Peter Braspenning made this possible. Also his assistance on Artificial Intelligence matters in this thesis is greatly appreciated. Harry Menninga and Maurice Houtsma are gratefully acknowledged for their careful reading of this thesis. Valuable assistance on the logical formalisms used in this work was given by Dr. Frans Voorbraak of the University 
of Amsterdam.

I am also very grateful for the personal help in difficult times, provided by Dr. Robert Visée and Peter Braspenning. 


\section{Directions for reading}

The summary on page 257 describes the main topics of the thesis. The summary is provided in the English language and the Dutch language. The outline of section 3.4, page 55 describes the contents of the chapters. Basic terms are given in section $\mathrm{A}$, page 237.

The work is divided into four parts: I) an introductory part, II) an analysis part that describes the formal properties and structure of Tactical Plan Recognition, III) a part that describes the design of two new Tactical Plan Recogmition algorithms and the last part (IV) gives a description of a demonstrator and the final conclusions. Each part ends with a chapter with a summary of that part.

In the following table the sections concerning the some important topics of the thesis are given.

\begin{tabular}{ll} 
If you are interested in: & read section or see page \\
\hline the most important conclusions & page 3.4 ,page 117,page 167, \\
& chapter 1.3 \\
planning & sections $6.1,6.2,7.3$ \\
generic task models & sections $2.1,2.2 .5$ \\
command and control systems & sections $1.1,2$ \\
TPR algorithms & sections $8.2,9.2$ \\
Haskell & section 8.1 \\
\hline
\end{tabular}




\section{Contents}

I Introduction to Tactical Plan Recognition

1 Introduction $\mathbf{5}$

1.1 Military operations . . . . . . . . . . . . . . 5

1.2 History . . . . . . . . . . . . . . . . . . . . . . . 9

1.3 The planning world . . . . . . . . . . . . . . . 11

1.4 The military world . . . . . . . . . . . . . . . . 14

1.5 Plan Recognition research . . . . . . . . . . . . . . . 16

1.6 Applications of plan recognition . . . . . . . . . 18

2 Tactical Plan Recognition (TPR) research 21

2.1 Generic Task Models . . . . . . . . . . . . . . . . . . . 21

2.2 Command and Control models . . . . . . . . . . . . 22

2.2 .1 The JDL model . . . . . . . . . . . . . . . . . . 22

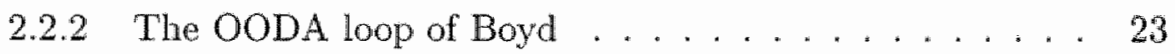

2.2 .3 Boasson's model based model ........... . . 25

2.2 .4 The MATIS architecture . . . . . . . . . . . . 25

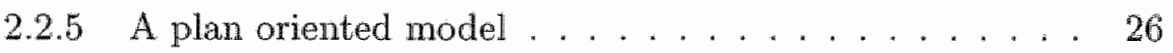

2.3 Examples of Tactical Plan Recognition . . . . . . . . . 28

2.3 .1 Civil aviation example . . . . . . . . . . . . . . 29

2.3.2 The air force example . . . . . . . . . . . . 30

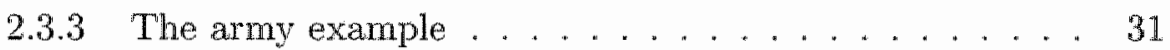

2.4 Tactical Plan Recognition research . . . . . . . . . . . 35

2.4.1 The first automated Tactical Plan Recognition system . 35

2.4.2 Systems for identified observations . . . . . . . 38

2.4.3 Systems for unidentified observations. . . . . . . . . . . 39

2.4 .4 Systems for learning doctrines . . . . . . . . . . . . 42

2.5 Possible new applications . . . . . . . . . . . . 43 
3.1 Discussion of Tactical Plan Recognition research . . . . . . 49

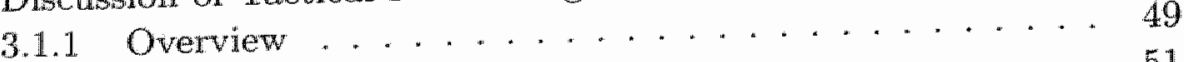

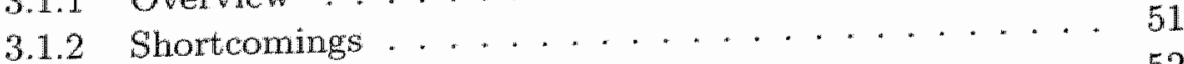

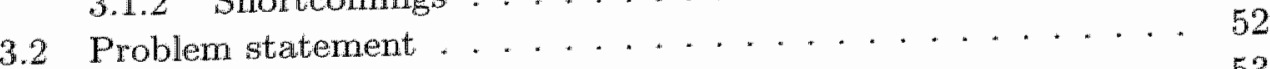

3.3 Addressing the problem statement ........... 53

3.3.1 Research approach .......

3.3.2 Multiple Hypothesis Tracking for TPR . . . . . . . 54

3.3.3 Application of abduction to TPR .......... 54

3.4 Outline of this thesis ............... 55

II Analysis of Tactical Plan Recognition 61

4 Introduction to Plan recognition

4.1 Classifications of Plan Recognition problems......... 63

4.1.1 Characteristics of plans ............ 63

4.1 .2 Classifications of Plan Recognition ......... 65

4.1 .3 Overview of Plan Recognizers . . . . . . . . . 66

4.2 A formal introduction to Plan Recognition . . . . . . . . . 68

4.2 .1 Formal definition . . . . . . . . . . . . 68

4.2 .2 Closed World Assumptions . . . . . . . . . 70

4.2 .3 Plan hypotheses . . . . . . . . . . . 70

4.3 Uncertainties of observations and plan execution . . . . . . 72

4.3 .1 Plans, states and actions.............. 73

4.3 .2 The observations . . . . . . . . . . . . 74

4.3 .3 The plan execution . . . . . . . . . . . 77

5 Deterministic and probabilistic Plan Recognition $\quad \mathbf{7 9}$

5.1 Deterministic Plan Recognition . . . . . . . . . . . . 79

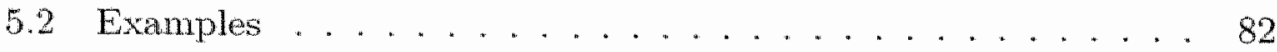

5.2 .1 Plan hypotheses . . . . . . . . . . . . . . 82

5.2 .2 Temporal considerations ................... 85

5.2 .3 Assignment hypotheses . . . . . . . . . . . . . . 88

5.3 Probabilistic Plan Recognition ... . . . . . . . . . . . . 94

5.3 .1 Reid's formula . . . . . . . . . . . . . . 95

5.3.2 Reid's formula for Tactical Plan Recognition ... . . . 98

5.3.3 A numerical example of Reid's formula . . . . . . . . . 98 
5.4 Propositions about hypotheses . . . . . . . . . . . . 101

5.5 Soundness and completeness . . . . . . . . . . . 105

5.6 Optimal Tactical Plan Recognition . . . . . . . . . . 105

5.6 .1 The optimization problem . . . . . . . . . 106

5.6 .2 Optimal kinematic estimation . . . . . . . . . 107

5.6 .3 Optimality over several scans . . . . . . . . . . 108

6 Generic Task Models for Plan Recognition 109

6.1 Reactive planning. . . . . . . . . . . . . . . . . . 109

6.2 Distributed planning and plan recognition . . . . . . . . 110

6.3 Analysis aspects . . . . . . . . . . . . . . . . . . 111

6.4 Generic Task Models . . . . . . . . . . . . . . . . . . . . . 111

6.4.1 Plan Recognition with identified observations: the domain problem ................... 111

6.4.2 Plan Recognition with unidentified observations: the domain problem . . . . . . . . . . . 113

\section{Design of Tactical Plan Recognition algorithms 119}

7 Design of the tasks 121

7.1 Examples recalled . . . . . . . . . . . . . . . . 121

7.2 The design of the Problem Definition task . . . . . . . . . 123

7.2.1 A Goal recognition knowledge base . . . . . . . . . . 124

7.2.2 Soundness and completeness of a forward-chainer . . . 126

7.2.3 Computational complexity of the forward-chainer . . . . 127

7.3 The design of the Planning task . . . . . . . . . . 127

7.3.1 Different granularities . . . . . . . . . . . . . 128

7.3.2 Planning algorithms . . . . . . . . . . . . . . 129

7.3.3 Soundness and completeness of Planners . . . . . . . 132

7.3.4 The complexity of Planners . . . . . . . . . . 132

7.3.5 Evaluation of planners .............. 133

7.4 Kinematic estimation . . . . . . . . . . . . . 133

7.4 .1 Purposes of kinematic estimation . . . . . . . . 134

7.4 .2 Options for filtering leg variables . . . . . . . 135

7.4 .3 Mean reverting process ............ 137

7.4 .4 Constant velocity along planned leg . . . . . . . . 139

7.4.5 Analysis of the two estimators ........... 140 
observations algorithm

8.1 The Haskell programming language . . . . . . 143

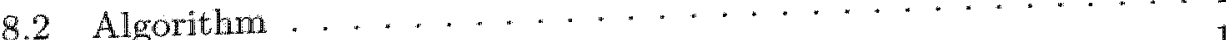

8.2.1 Consistency check 147

8.2 Propositions $\ldots \ldots \ldots . \ldots 147$

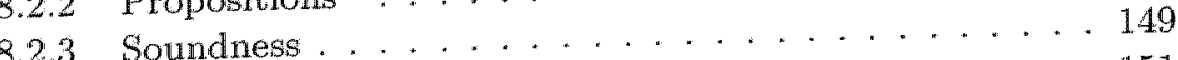

8.2 .4 Completeness . . . . . . . . . . . . . . . 151

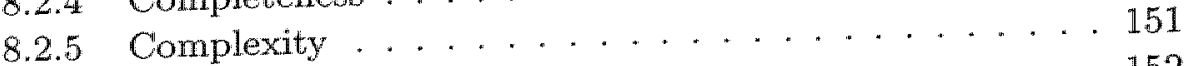

8.2.6 Hypotheses assessment . . . . . . . . . . 152

9 Unidentified observations algorithm 153

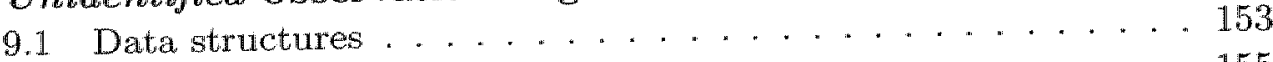

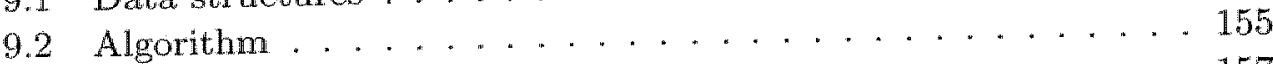

9.2 .1 Functions . . . . . . . . . . . . . 157

9.2 .2 Soundness and completeness ............ 160

9.2 .3 Complexity ..................... 163

IV The Demonstrator and conclusions 169

10 The Demonstrator 171

10.1. The architecture of the Demonstrator . . . . . . . . . 172

10.2 The design of the Simulator . . . . . . . . . . . 175

10.2 .1 Class diagram of the Simulator's Basic Database . . . 170

10.2.2 The class diagram of the Simulator's Track Database . . 177

10.2.3 The numerical mathematics module . . . . . . . . 179

10.3 The design of the Human Computer Interface . . . . . . . . 179

10.3.1 The operational display . . . . . . . . . . . 179

10.3.2 The operational control . . . . . . . . . . 181

10.3.3 The plan information display . . . . . . . . . 182

10.4 The design of the Tactical Plan Recognizer . . . . . . . . . . 184

11 Experiments 195

11.1 The army example . . . . . . . . . . . . . . 196

11.1.1 Examination of assignments . . . . . . . . . . 199

11.1.2 Examination of probabilities . . . . . . . . . 210

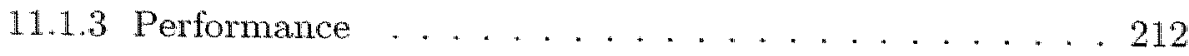

11.1 .4 Tuning. . . . . . . . . . . . . . . . 213 
11.2 The littoral scenario . . . . . . . . . . . 214

11.2.1 The best assignment hypotheses before tuning . . . 217

11.2 .2 Tuning . . . . . . . . . . . . . . 220

12 Evaluation $\quad 225$

12.1 Interpretation of the experiments . . . . . . . . . 225

12.2 Tuning process . . . . . . . . . . . . . . . . 226

12.3 Software engineering approaches . . . . . . . . . 227

12.3.1 Haskell and the Tactical Plan Recognizer . . . . . . 228

12.3.2 Simulator, Human Computer Interface and Java/Swing 229

13 Conclusions

13.1 The problem statement revisited ... . . . . . . . 231

13.1.1 A direct and optimal method . . . . . . . . . 232

13.1.2 A feasible method: the algorithm . . . . . . . 232

13.1.3 A sound and complete algorithm . . . . . . . 232

13.1.4 Conclusion on the algorithm development . . . . . 233

13.2 Future research . . . . . . . . . . . . . . . . 233

$\begin{array}{ll}\text { A Basic terminology } & \mathbf{2 3 7}\end{array}$

B Requirements on a TPR product $\quad 239$

C XML schema for assignment hypotheses $\quad 241$ 
CONTENTS 


\section{Part I}

\section{Introduction to Tactical Plan Recognition}




\section{Chapter 1}

\section{Introduction}

Human planning and human plan recognition constitute a human's reasoning about actions. This is a major mode of human reasoning. Plan recognition generally concerns recognition of other humans' plans from observations of the properties and actions of that person or group of persons and is a kind of inverse planning problem.

Recognizing the plan(s) underlying observed human behavior is an important problem in many contexts. Traditionally, automatic plan recognition has been applied in areas like story understanding and user modelling. In this thesis formal definitions and algorithms for the recognition of enemy plans are proposed. We will focus on military applications, where Tactical Plan Recognition plays a crucial role, but obviously this task is relevant whenever one has to deal with adversarial agents ${ }^{1}$.

\subsection{Military operations}

The topic of this thesis is Tactical Plan Recognition. It is a sub task of a Command and Control system. Command and Control systems manage the operations of civil or military agencies. However, they are mostly developed and applied for military applications. That is, naval, army, air force applications. In some civil agencies, such as the police and the fire-department, Command and Control systems are also found.

\footnotetext{
${ }^{1}$ In our military context, an agent can be a human (and its equipment) or a group of humans that operate as an entity. But it can also be a unmanned vehicle. An agent is often thought to act somewhat 'intelligent'.
} 
A well known example of an early Command and Control system is a "operations room' of the Royal Air Force during the Battle of Britain. In this room one found a large geographic map of a section of England. On the map, all squadrons of the British air force, as well as detected German flights, were depicted by scale model airplanes. These models were placed upon the map at the currently known positions of their squadrons. The actual positions of these flights were communicated regularly by means of telephone messages. These messages were based up on human observations and radar detections of the aircrafts. Whenever a new position was communicated, the scale models would be moved to that new position on the map. Standing on a balustrade, above and around the map, commanding officers examined the map. The map provided an overview of the movement of enemy flights and the current position of British scuadrons. Based on this overview, the actions of the British squadrons were planned by these officers. To execute the plan, the Royal Air Force fighters were directed by radio to their successive targets.

After this introductory example, some general statements about military operations will be given. In a military world we find two types of organizations: the own forces and the ackersarial enemy forces. These forces are military organizations that are organized in units. The military units of the own forces cooperate to achieve some shared goals. They are opposed in their efforts by enemy forces. The goals and also the means and restrictions on the amount of force that may be applied during the operation are stated in a document called the mission of the own forces. Generally, the mission is stated by the political rulers of the country of the own forces. To achieve the goals of the mission (with the assigned means) an operational plan is made by the military. Such a plan is also called a course of action (COA). The execution of such a tactical plan is called a military operation. The operation can vary significantly in size. It can be a part of a larger operation or be an isolated operation. An example of a large operation is the conquest of Iraq by coalition forces. Parts of the Irac campaign were the battle of Bagdad by American forces and the battle of Um Qasr by British forces. In figure 1.1 a class diagram in the Unified Modelling Language (UML) relates the different concepts. Here the concepts are stated by boxes, their relations by arrows between the boxes.

Of course, the operation faces some difficulties. There are only limited resources available to execute the operation, i.e. the resources are constrained. Also, the opposing Iraqi forces, such as the republican guard, tried to frustrate the efforts of the coalition forces. Their reactions could not be fully antici- 


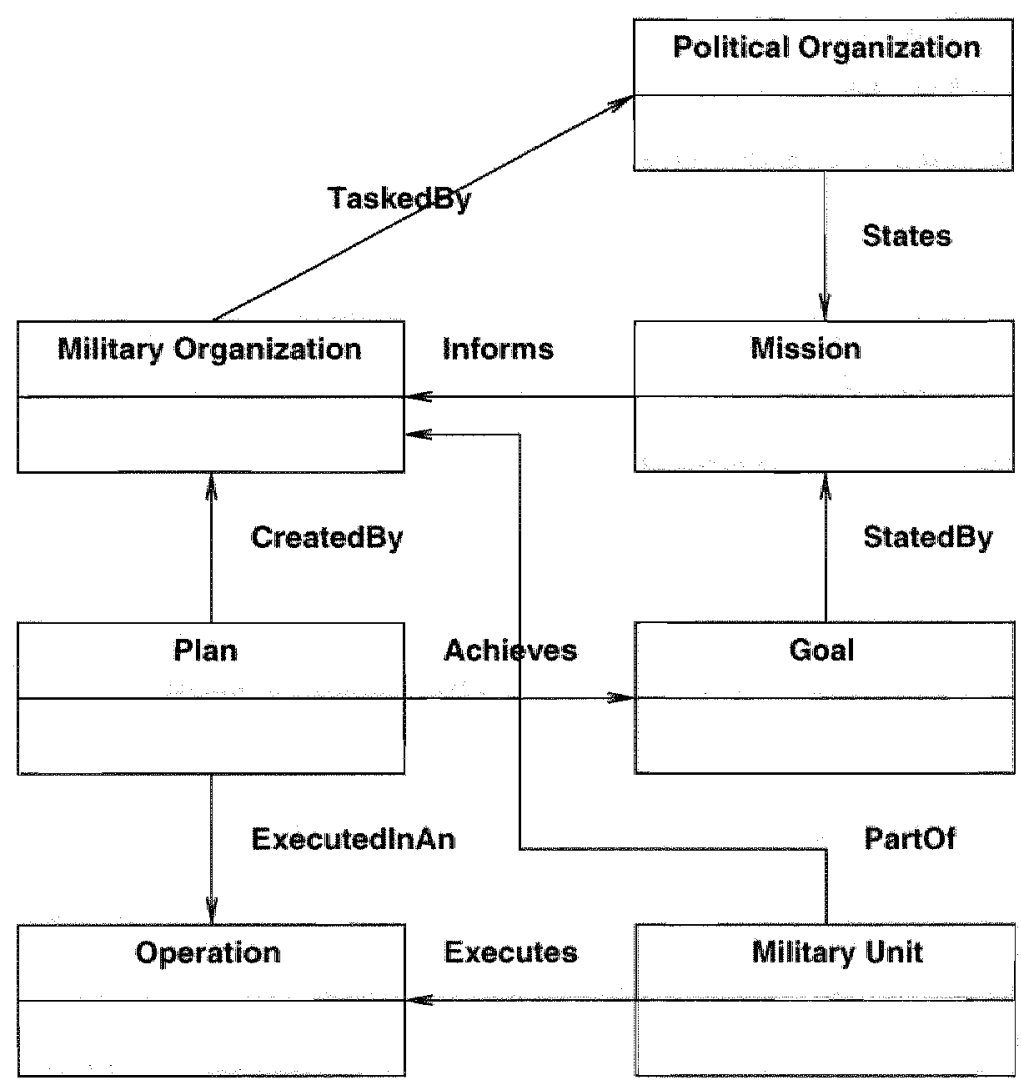

Figure 1.1: UML class diagram of military plans and their execution. 
pated during the planning of the operation. The weather conditions also can influence the operation. During the march towards Bagdad, the American Forces were delayed by a sand storm.

To landle the unexpected events, the operation is controlled by the military command. The most important tasks of the command are to do own forces' planning and replanning in case of unexpected events. Having established the need of command, we can now introduce a description of a Command and Control system (from Harris [34]):

Command and Control systems are complex organizations of people and equipments which have to function in a coordinated way to achieve their objectives.

One can also take the view that only the equipment constitutes the Command and Control system, as is done in the following quote (also from Harris [34]):

Command an Control $\left(C^{2}\right)$ Systems are the management infrastructure for defence and war or for any other large scale complex dynamic information/resource management system

Both definitions do not take in account, the large body of procedures, that is compiled for operating such a Command and Control system.

During the planning and during the operation, the military command needs to have an assessment of the military situation: the tactical situation. Situation assessment implies knowledge of the composition of the units, of enemy forces' location and their possible plans. The assessment task is performed by intelligence units.

In the military, tactical plans are distinguished from strategic plans and operations. Whereas strategic plans are long term plans and influence the actual battle indirectly, tactical plans are rather short term plans and are directly related to enemy units. Strategic plans may have as a goal to destroy the enemy's military industry, communications network and logistic capabilities. Tactical plans may concern an attack on a particular enemy unit to take their position.

So a military Command and Control system is faced with two main tasks: enemy plan recognition and own forces'planning. The first task is enemy plan recognition based on the assessed military situation. The military situation is also called the tactical situation. The tactical situation can only be assessed partially and has many uncertainties. It concerns knowledge of: 
- enemy objects and their operations,

- the enviromment, being the geography and weather, and

- descriptions of the enemy's and own forces' actions in pre-defined military situations. These actions are called doctrines in military parlance or plan templates in the Artificial Intelligence world.

- hypothetical missions of the enemy.

This partial knowledge is obtained from intelligence information, sensors and link information, reconnaissance, etc.

The second task is, based upon the recognized enemy's plans and your mission, to do own forces planning. In order to perform these tasks, models of the environment and own forces and enemy's units are available. The world of military combat systems is dynamic, and often only uncertain and incomplete information is available. Here planning must be conditional and reactive. In conditional planning (also called contingent planning) the world is monitored during plan execution and conditional plans are executed when their condition, i.e. a particular tactical situation, is satisfied. Reactive planning means that the plans are adapted to unexpected events and actions during plan execution when these plans are not apt anymore. The complexity of the real world and its distributed resources and objects may promote distributed problem solving methods.

Currently, Command and Control systems are large computer networks that run computer applications. The applications often have been developed with scientific methods. Application of the scientific method for Command and Control systems started in the first world war. The history of the application of scientific methods to Command and Control systems is elucidated in the next section.

\subsection{History}

Before the first world war, scientific methods were only applied to the constructions of weapons, but not to the actual warfare. The first effort to model a military conflict mathematically was made during the first world war by F.W. Lanchester [46]. He defined a set of differential equations, called the Lanchester models of warfare, with the number of combatants as a function of 
the duration of a battle. This model predicted the duration and the outcome of a battle. Since Lanchester's model a lot of research on warfare models has spawned off, but was always based on his differential equations.

Another important branch of military research concerns specific management problems of a military operation. For isolated and well defined parts of the planning of military operations, scientific methods were developed during the second world war. In Winston [97] the origin of this branch is described as follows.

During the second world war British military leaders asked scientists and engineers to analyze several military problems: the deployment of radar, the management of a convoy, bombing, logistics and antisubmarine and mining operations. The application of the scientific method to military operations was called operations research in which (often optimal) solutions for all kinds of management problems were sought. After the second world war computers became available and the first algorithms for operations research were developed.

A prominent example of operations research is linear programming. In 1947, George Dantzig developed an efficient method, the simplex algorithm, for solving linear programming problems. It was applied during the Berlin airlift shortly after the second world war. Soon after the war, operations research addressed all linds of management problems; often commercial ones.

As far as plan recognition (the topic of this thesis) is concerned: in historic times the tactical situation was only perceived through intelligence messages of human observers. Thus, the information was often very limited. During the end of the $19^{\text {th }}$ century, human airborne reconnaissance became possible by putting observers in balloons and in the first world war airplanes were employed for this purpose. With the advent of modern sensors, such as radar, these sensors were rapidly applied to generate information for situation assessment. During the second world war situation assessment was done manually in the control rooms. After the second world war computers became available and since the sixties and seventies, situation assessment was supported by computerized data processing systems.

So, with regard to the planning task, there was some progress in its definition and the application of scientific methods. But the situation assessment task was not well defined. Only supporting systems, such as large graphical 
computer displays and databases were developed. The intelligence task itself could not be automated. In 1987 it was observed by Harris and White [35] that:

There is no adequate theory for Command and Control (i.e. the situation assessment) nor a fundamental methodology for its design.

The situation assessment task's complexity defied computer automation and relied on human effort until recently. The term situation assessment denotes having some 'understanding' of the 'tactical situation' and is a rather vague term. Azarewicz [7] was the first author who recognized that plan recognition is an important part of situation assessment. Plan recognition was already a well established branch of research of the Artificial Intelligence community at the time. The history and body of research in the field of Tactical Plan Recognition is further described in section 2.4.

This concludes the brief history of science and warfare. In the following sections first the planning world and then more specifically the military world including its Command and Control systems will be further introduced.

\subsection{The planning world}

Planning is done in a world with related planning objects. A planning object can be anything, it can be one human, a group of humans and their equipment or concern automated machinery. So the objects may be human and intelligent but also inanimate objects. If they act 'intelligent" these objects are also called agents. Another important agent is the one that devises a plan, this agent is called the planner. The planner desires that the state of the planning world is different from what it is at the time of planning.

At any point of time the planning world is in a particular state, that changes by actions. Actions are intentional, because they are invoked by a planning object and serve some purpose. The world is also changed by events that are non-intentional and are not planned. When a plan, that consists of several actions, is executed, then the world changes from its initial state to the desired state of the planner. The desired state is called the goal or goal state. These concepts are related to each other by the UML class diagram of figure 1.2. A new feature in this figure are the 'IsA' relations which denote a generalization/specialization of a concept. 


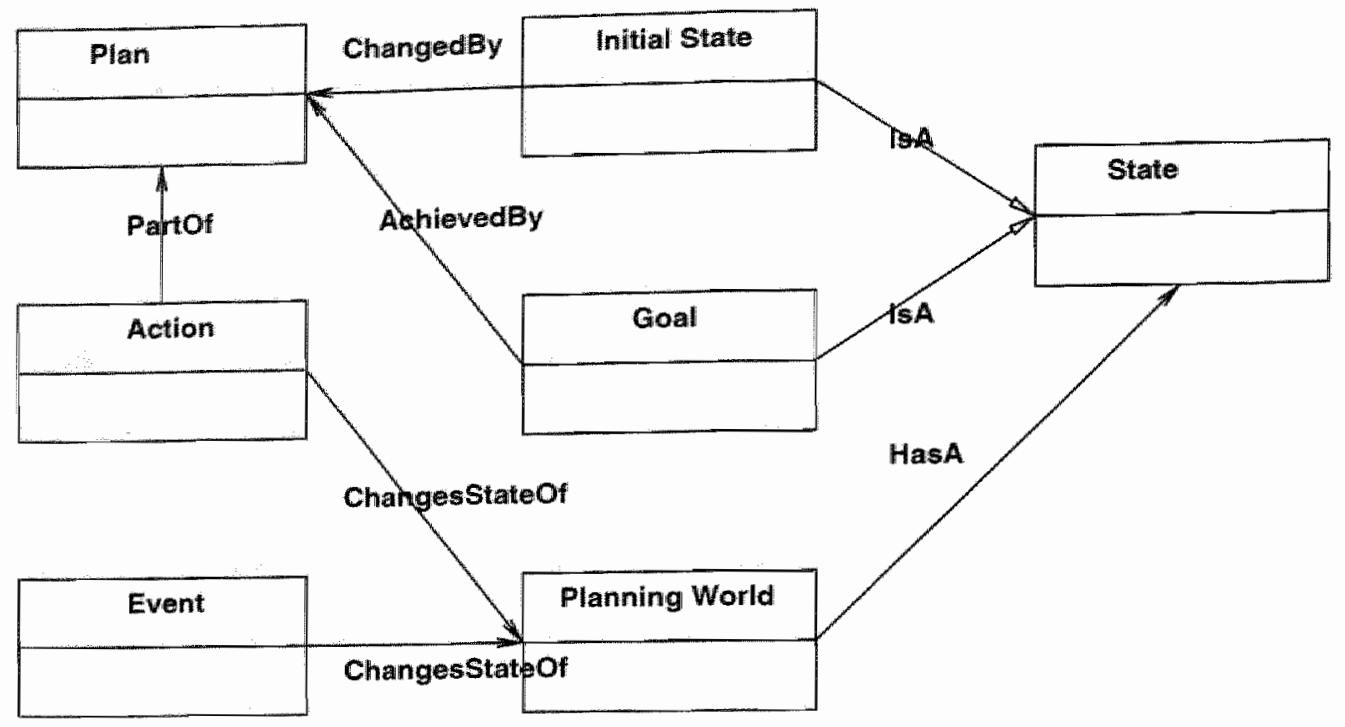

Figure 1.2: UML class diagram of the plamning world.

The planner is restricted in the number of degrees of freedom in the planning world. The number of degrees of freedom is the number of plan parameters that can be varied. The range of the parameters is limited by constraints. Constraints exist because there are limited resources that can be applied for achieving particular goals. Conflicts accur during planning or during plan execution and can prevent reaching particular goals, e.g. if a constraint is violated.

A more elaborate overview of planning is found in, for example, the textbook of Russel and Norvig [76]. From this book comes the following shopping example:

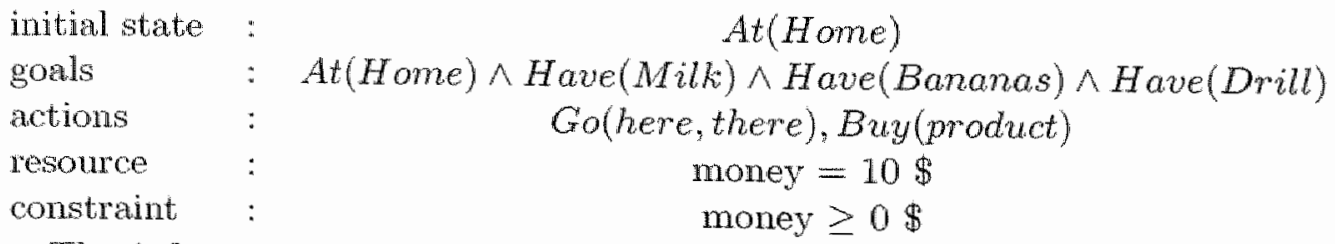

The information above consists of logical statements like At(Home) or compound logical statements like: $A t($ Home $) \wedge$ Have $($ Milk $) \cdots$ and numerical equations. Symbolic or numerical variables are printed in lower case: here, 
there are symbolic variables that can have as value Home and Shop and other places, money is a numerical variable. The initial state describes that a person is initially at home. The person has some goals: to have milk and to have some bananas and to have a drill. Generally relevant actions are described as the person going from one place (here) to an other place (there) and by stating that the person can buy some product. Of course, shopping is constrained by the amount of money a person has. In this case the person has initially 10 dollar and requires to stay out of debt: his amount of money must be greater than zero dollars.

Real world planning problems and, therefore, also real world plan recognition problems differ significantly from toy worlds planning and toy world plan recognition problems. Examples of toy worlds are the blocks world in planning and even the above shopping example can be classified as a toy world, even though it seems realistic at first. To clarify this, we first describe two significant characteristics of the real world.

First, the real world is inherently distributed. Some planning problems concern distributed resources and agents and/or are so complex (intricate, large scaled), that the planning problems have to be solved by a number of distributed, cooperating agents together. Here coordination of the activities of the agents is required, but still allowing for sufficient autonomy for the individual agents. Only when the agents have sufficient autonomy, they can operate efficiently and are not blocked by communication or waiting for synchronization.

Secondly, the real world is dymamic; during plan execution unexpected events can change the world such that a plan may no longer be applicable or sufficiently optimal. It contains conflicts (i.e. actions that prevent goal achievement) or it should be improved. Here reactive planning (which is in fact rather acute re-planning) is required. In reactive planning, the world is monitored for unexpected and expected events or states during the plan execution. If a plan is not applicable to the new situation of the world any more or might be improved significantly, it is adjusted to the new situation by means of reactive planning.

Though the shopping example concerns a distributed world, the amount of objects is very limited. Moreover, the 'outside' world's dynamics are not taken in account, therefore it can be argued to be classified as a toy problem.

Now the planning world has been introduced, we will examine a specific type of planning world that is relevant for our Tactical Plan Recognition study: 
the military world.

\subsection{The military world}

The military world addressed by a Command and Control system is a professional world. A professionall world is related with a specific activity with well defined concepts. It is abstracted from a real 'outside' world. Note that a professional world differs from a real world. A real world is fairly complex and may entail commonsense reasoning. Commonsense reasoning is not well defined and rather difficult to model by a computer program. In the case of a professional world, the objects and agents and their relations and states and actions are well defined. So the professional knowledge can be made explicit with knowledge acquisition techniques. This is the first prerequisite for developing computer applications to for problems in this field.

Of course the military world is classified as a real world, that is fairly complex in itself. However, here also some additional problems occur. These are listed below:

1. Multiple agents and objects, independent or interacting, and a large number of agents and objects involved in the military world. In the naval domain the number of objects and agents may be in the range of $\mathbb{1}$ till 100. In modern larger scaled army conflicts, the number of observed objects and agents to be considered may be several hundreds.

2. Multiple independent plans or related plans. At the same time several plans may be executed by the combatants of the military world. The executed plans may be part, of a larger plan, but can also be independent of each other.

3. Covertness. The enemy forces will try to cover their operations as much as possible. But also the sensors and observers only have a limited view of the objects and agents in the military world. This results in various forms of uncertainty about the observations:

(a) An uncertain identification of the observation. In the military world, the observed objects that are involved in the observations are not identified. One does not know if two objects of different observations are the same. 
(b) Uncertainty about the truth of an observation. The observation itself may only have a limited degree of belief. This happens when one does not trust the source or the communication channel completely. In the military world the belief in an observation can be qualified as either Confirmed, Positive, Probable or Not Probable 2 .

(c) uncertainty in the parameter values of the observation. The parameters concern attributes of the object(s): its radar cross section, shape, etc. and the kinematic state vector (position, speed) of the object or parameters of actions that are performed by the object.

4. The observations originate from various observers and sensors. So the plan recognizer has to fuse observations from all these sensors and sensor data fusion techniques have to be applied. Note that a human observer differs significantly from automated sensors, such as radar. They differ in terms of their datia rate as well as in their observation characteristics.

5. Feints executed by enemy forces. the enemy forces will perform feints in order to mislead the own forces' plan recognizers.

6. Plans may be executed erroneously. Errors are often made on the battlefield and even fratricide occurs here. This has to be accounted for during plan recognition.

7. Partial or no achievement of goals. The goals of a plan may not be achieved at all or only partially.

In the subsequent parts of the thesis, we will show that many of these uncertainties can be taken into account in doing Tactical Plan Recognition. A model can be devised that integrates many of the above mentioned characteristics. However, many of the parameters of the model are not known exactly and tuning is required to find good values for unlnown parameters.

Continues now by having a first look at the field of plan recognition.

\footnotetext{
${ }^{2}$ These measures are often based on the reliability and number of sources from which the information originated. A Confirmed piece of information has been received from two or more reliable and independent sources. A Positive message has only been received by one reliable source, etc.
} 


\subsection{Plan Recognition research}

Plan recognition was first recognized as a problem as such, by Schmidt et. al. [78] in their work on the BELIEVER system. In their paper they state: "The BELIEVER system is a psychological theory of how human observers understand the actions of others". The understanding is described as: "attributing intentions, beliefs and goals to an (other) actor". But apart from goals, Schmidt also wanted to deduce a complete plan. The BELIEVER system was implemented by a number of cooperating expert systems ${ }^{3}$ that captured heuristic goal and planning rules used by humans. The expert systems first state likely goals, where after a number of possible plans (also called plan hypotheses) are generated. These plans cars achieve the recognized goals. This approach of generating plan hypotheses is also used in this research.

The first attempt to define a logical framework for Plan Recognition, was in Kautz's and Allan's generalized plan recognition paper in 1986 and his chapter in Allan's book "reasoning about actions" of 1991, [44, 4]. They start with a taxonomy of actions that are related to each other through generalization relations ('IsA') and composite relations ('PartOf'). To ensure that the action taxonomy is an exhaustive and exclusive description, they specify two closed world assumptions ${ }^{4}$ :

Assumption 1.1 Kautz's and Allan's first closed world assumption: the (by the plan recognizer) known ways of performing an action are the only ways to perform the action.

So only the doctrines that are modelled in the background knowledge of the plan recognizer are assumed to comprise the complete knowledge necessary to do plan recognition.

Assumption 1.2 Kautz's and Allan's second closed world assumption: all actions are purposeful and all possible reasons for performing an action are known.

\footnotetext{
An expert system is a computer program that models hurnan reasoning knowledge in some limited and often professional field. Expert systems are a spin-off of the field of research of Artificial Intelligence.

A Knowledge Base (KB) of logical rules and statements is complete if every atomic statement that can be made in the logical language and its negation can be derived from the $K B$. Often the $K B$ is not complete and needs to be augmented with some assumptions that 'close' it. A well known assumption is the Closed-World Assumption (CWA): if an atomic statement is not avallablle or comprised in the $\mathrm{KB}$, then its negation is true.
} 
These closed world assumptions can be stated in a logical reasoning scheme for closing a set of statements. By performing this closure, plan hypotheses are created by Kautz's algorithm. These two assumptions have also been applied. by later authors.

An important link with the classical abduction way of reasoning (as defined. by C.S. Pierce) was recognized by Lin and Goebel [50]. Obtaining a solution to a plan recognition problem is similar to drawing inferences using classical abduction, which can be described as the process of conjecturing explanatory hypotheses. An example of abductive reasoning is: if you are told that all ravens are black $(\forall x(R(x) \Rightarrow B(x)))$, and you observe that a bird $b$ is black $(B(b))$, then you can conjecture that $b$ is a raven $(R(b))$.

Consequently, as abduction and induction are both concern hypotheses, techniques from machine learning were applied by Bauer [11] in 1996. In inductive reasoning one finds a set of examples, specified as the pair $(x, f(x))$, where $x$ is the input and $f(x)$ is the output of a function that takes $x$ as input. From the set of examples one returns a function $h$ that approximates $f$. For example: from a set of observations of birds $b 1$ and bird $b 2$ $:(R(b 1), B(b 1)),(R(b 2), B(b 2)$, one can infer that $\forall x(R(x) \Rightarrow B(x))$. In our plan recognition case, the observations are the $x$ 's and the actual plan results in the observations. $h$ is a plan hypothesis, an approximation of the actual plan $f$. Particularly techniques from inductive concept learning were applied by Lesh and Edzioni in 1995 [48].

A view that recognizing plans is much like parsing a text was first given by Sidner (as early as 1985) [80]. It is quite obvious that the non-terminals of a grammar correspond with atomic actions that are observed. Legal sequences of these actions are stated by grammar rules for non-terminals. This approach was further explored by Villain [92] in 1990. Villain established a correspondence between a restricted form of Kautz's framework and parsing of a computer language. He could convert Kautz's action taxonomy by first encoding the composition part (Partof related actions) and then adding the abstraction part of the taxonomy.

But a purely logical framework does not model sufficiently uncertainties in the observations. Moreover, it gives a large number of plan hypotheses that cannot be ranked. Once hypotheses are ranked one can select the kbest hypotheses and as such one has a more manageable set of hypothesis. Since Kautz, several other authors have applied general techniques for reasoning about uncertainty (Bayesian nets, Dempster-Shafer belief functions and 
combination rule) in other frameworks for plan recognition. Bayesian nets were first applied to plan recognition by Calistri-Yeh [19] and Charniak and Goldman in 1991, [25]. Their work was succeeded by the work Pynadath and Wellman [71] and several other authors. Dempster-Shafer theory was applied to plan recognition in the area of natural language understanding by Carberry [22] in 1990 and by Bauer [10] in 1994 in the area of intelligent user interfaces.

\subsection{Applications of plan recognition}

Plan recognition has been around for some time now and a wealth of research is available; posing solutions for a number of applications. The most important applications of plan recognition are:

- Psychological modelling: see the publication of Schmidt, et.al. [78] that explores plan recognition as an intersection between psychology and artificial intelligence. Schmidt worked on the BELIEVER system that was a computerized psychological model of plan recognition. The system maintained several databases with information about the environment, the observed person and the perceived plans. Plan templates consisted of operators that were somewhat similar to STRIPS (for STRIPS see [30] or [76]) operators, and Schmidt devised a generate and test algorithm to pose plan hypotheses.

- Story understanding: in a story, written descriptions of the state of the world and of the story's agents' actions are described. In story understanding a program attempts to infer the plans that, when executed, may cause the action descriptions. Once the plan is recognized, the story understanding program can produce a paraphrase or a summary of a story and answer questions about the story. The first outline of a computational theory of story understanding was Charniak's thesis of 1972 [24]. The first story understanding implementation was Cullingford's SAM (Script Analyser Mechanism) of 1978, [27, 28], applying Schank's and Abelson's [77] scripts. Scripts are (memory) structures that organize knowledge about stereotypical situations, such as dining at a restaurant, by stereotypical sequences of actions.

- Natural language understanding: here verbal descriptions of actions and the world are available. Note that the verbal language differs significantly from written language, such as found in the story understanding 
applications. The plan recognizer compiles a plan of a human that explains the utterances of the person. Dempster-Shafer theory was applied in plan recognition in the area of natural language understanding by Carberry in 1990 [22]. Another author in this field, Ingrid Zuckerman [103], gives a logical framework, that applies STRIPS like operators to infer "interpretations" of utterances, augmented by a likelihood calculation of the interpretations, based on Bayes' formula.

- Automated driving: in a car driving environment, the drivers have to coordinate their driving plans with each other, in order to prevent collisions. The coordination relies on visual observations and audio signals of other drivers actions. So the communication is restricted in this area. In this it differs from the applications for agent coordination. Based on these observations a driver recognizes other driver's plans and modifies its own driving plans. For an example see the article of Pynadath and Wellman in 1995 [71].

- Intelligent computer user interfaces: here the plan recognizer attempts to infer the user's plan(s) by his/her interactions with the computer. When the interface program knows the user's plan(s), it can give advice to the user on how to attain the goals of the plan. A pioneering work here is that of Candace Sidner in 1985 [80]. Mathias Bauer applies DempsterShafer theory in his 1994 paper [10]. Modelling the knowledge of a user to support user plan recognition has been done by Liliana Ardissono in $1996[5,6]$.

- Program understanding: here the plan behind a piece of computer language code is recognized and thus its goals are understood. Program understanding is reverse engineering of legacy code. An early publication is that of Wills in 1990 [96], which was followed by several publications from Alex Quilici, Steven Woods and Qiang Yang, [72, 73, 101, 100].

- Multi-agent coordination: Huber and Durfee [40], first applied plan recognition for collaborative planning in 1993. The idea is that the agents recognize each others plans by observing them. Thus, the amount of communication of plans between each other is reduced. The work of Huber and Durfee relies mainly on heuristics. VanWie also used plan recognition for coordination in his paper of 1998 and thesis of 2001 $[89,90]$. He proposes to augment an agent with a Bayesian net plan 
recognition system to infer an other agent's plan. Based on the other agent's plan, the agent can adjust its coordination mechamism to it. He also models the probable beliefs of other agents. He applies this agent in the Multi User Dungeon (MUD) game.

- Tactical Plan Recognition: the body of research of this field will be elucidated in section 2.4 about research in Tactical Plan Recognition.

Currently there is a fainly large body of research concerning plan recognition and plan recognition applications. However, there is not yet any known application that has found some widespread use, commercially or otherwise.

Now we have looked at the plan recognition research and its applications in general, we will focus at the military application at hand in the next chapter. Here, first Command and Control systems, in which a Tactical Plan Recognition application will have to function, are examined. 


\section{Chapter 2}

\section{Tactical Plan Recognition research}

This chapter introduces the Tactical Plan Recognition problem in more detail. It starts with an examination of some of the characteristics of Command and Control systems in order to define a context of the Tactical Plan Recognition task. Then some simple but illustrative examples of tactical situations are given and the process of plan recognition is described for them. The purpose is to get a first insight in Tactical Plan Recognition. Finally, a summary of past and current Tactical Plan Recognition research is given.

To somewhat formalize the previous statements about the Command and Control systems domain, a Command and Control system is described in this chapter by several Generic Task Models and architectures. First, a description of the method of the development of a Generic Task Model is given. Secondly, a number of general descriptions of a Command and Control system from literature are presented. Because these systems do not describe the interaction between planning and plan recognition, a new Generic Task Model is proposed. It encompasses both the planning and plan recognition tasks.

\subsection{Generic Task Models}

Generic Task Models were originally proposed. by Chandrasekaran in 1984 [23] and describe the sub tasks and the generic information flow between them for a class of problems. Some well known examples of problem classes are classification, heuristic classification, planning, reactive planning and diagnosis. 
The Generic Task Model provides insight in the structure of a problem class and can be instantiated to specific problems that are instances of the problem class. The problem classes can be organized hierarchically. For example, naval Tactical Plan Recognition and driver's plan recognition are instances of the problem class plan recognition.

Designing a Generic Task Model is not straightforward. Of paramount importance in developing a Generic Task Model is the level of abstraction at which to design the task model. A good rule for choosing the level of abstraction is to choose that level at which generic tasks relate with a distinctive body of literature and research, e.g. planning. In that case, for a generic (sub)task, algorithms and/or modelling tools are available from literature and can readily be applied to the instances of the generic task.

A Generic Task Model is not a formal description of a problem class. So its correctness can only be informally proved by a sufficient number of successful instantiations or by the insight it gives. Generic Task Models originate from the design of expert systems and are very well described in Tansley \& Hayball [87]: a book about the 'KADS' expert system development system. They also give a multitude of Generic Task Models.

After this introduction to Generic Task Models, we now can examine some generic descriptions of Command and Control systems.

\subsection{Command and Control models}

Because military Command and Control systems are the topic of numerous studies and system proposals, some Generic Task Models (though not always called ass such) for Command and Control systems are defined in the literature. Some interesting ones are elucidated below.

\subsubsection{The JDL model}

The most widely used model in the technical designer world of sensor data fusion systiems is that for data fusion and information fusion of the Joint Directors of Labs (JDL). One finds it in almost every course and textbook about sensor data fusion system design. JDL is an US organization of several military research laboratories. A draft revised JDL model was given in 1998 (see Bernstein [13]) in which hypothetical reasoning was stressed. The revised JDL model is the most interesting one for us, because hypothetical reasoning is 
the basis of the work of this thesis. The basic item to reason about in the JDL model are objects. An object can be a moving object, such as a vehicle or a static object such as a building. Also the level of detail, i.e. the granularity, of the object can vary. It can be a complete unit or an individual tank. The JDL model proposes the following successive levels of processing and reasoning:

- Level 0: Preprocessing/predetection: inputs are physical measurements of objects and the output is a measurements location and its attributes.

- Level 1: Single Object Refinement: the input are the measurement's location and attributes and the output are objects' behaviors, friend foe identification and the object's class. The output is based on sets of measurements that are thought to originate from the same object. Such a set is called a track. The track gives the behavior of the object in time. The process at level 1 is identical to tracking of objects.

- Level 2: Situation refinement: here the tracked objects are aggregated and refined. The output is a situation interpretation, that is the intentions and vulnerability of objects.

- Level 3: Implications/Threat refinement: the output here are enemy plans, also referred to as Courses of Action (COA).

- Level 4: Process refinement: this entails control of all the previous processes.

The JDL model is displayed in figure 2.1. The JDL model describes the structure of a Command and Control systems and our plan recognition task can be positioned at level 3. The model describes a Command and Control system at a high level and the tasks are not elaborated. The situation refinement function is described rather vague and no direct link with the field of research of plan recognition is made here. Neither is the interaction between planning and plan recognition recognized. Though this is understandable, as the model mainly concerns the sensor data processing part of a Command and Control system.

\subsubsection{The OODA loop of Boyd}

The OODA loop of Boyd, $[17,26]$, is very popular in the military world. It aims at all tasks in a Command and Control System. OODA is an abbreviation 
24 CHAPTER 2. TACTICAL PLAN RECOGNITION (TPR) RESEARCH

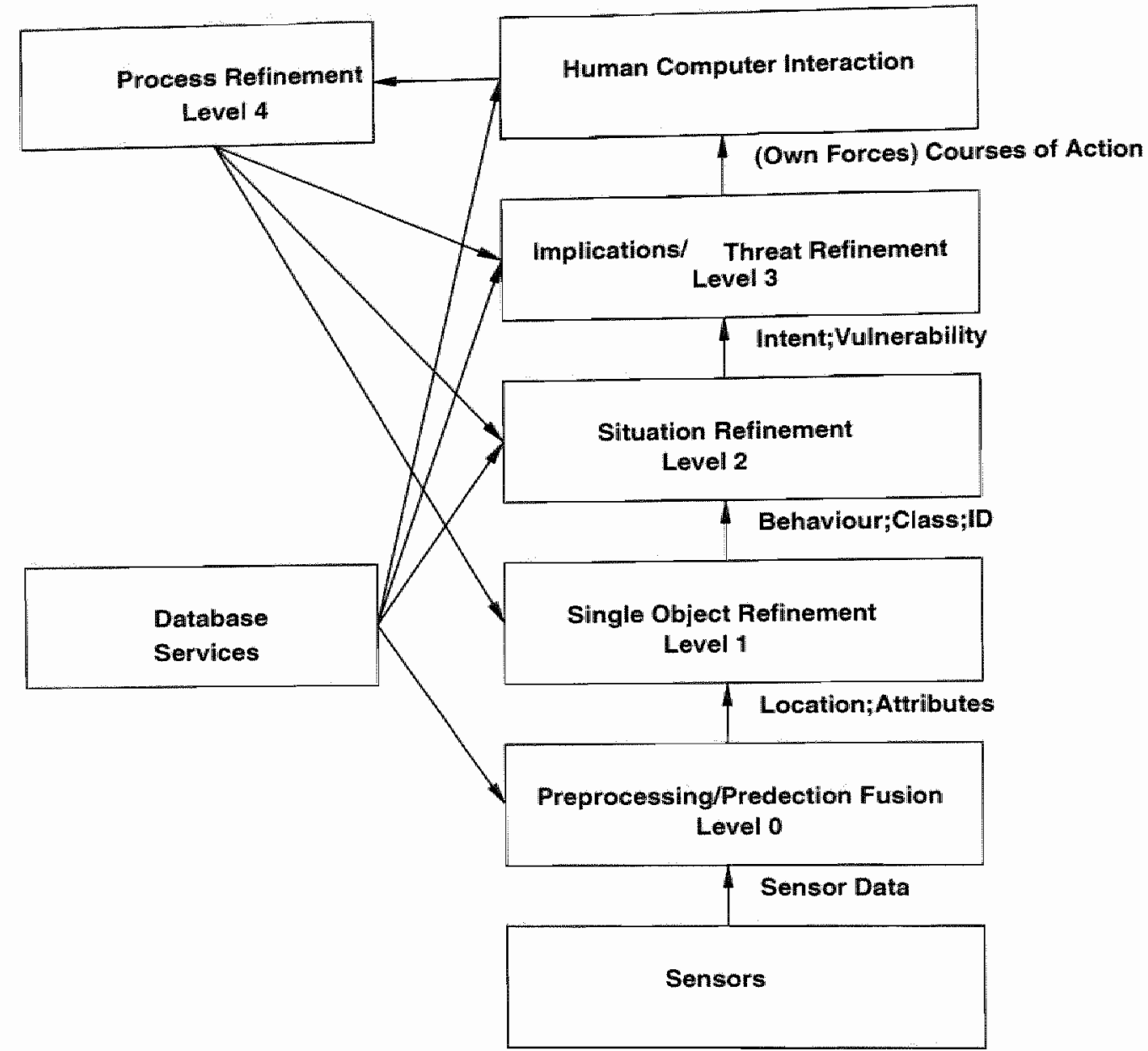

Figure 2.1: JDL Data fusion functional model. Adapted from Bernstein [13]. 
of Observe, Orient, Decide and Act. In the OODA Loop the Decision maker is the crucial element of command and control process. Boyd discerns the following main activities of the Command and Control, from [26]:

- Observe environment - to collect data about the surroundings and the status of enemy/friendly forces fused. The data is displayed in a common tactical picture.

- Orient - forms mental picture of situation by converting data into judgments about whats happening. The intelligence process plays a key role here. Derives basic situational awareness of battlespace.

- Decide - comes up with plan for course of action.

- Action - sets forth intent and issues orders to put the plan into action. Continually monitors execution of operations and gauge results.

The OODA loop gives a very general description of the processes and is used mainly for training officers. Though the OODA loop was designed for the military, it is now also applied in civil decision processes and psychological modelling. The model does not relate the main tasks with a specific body of scientific research and has been designed originally for practical use "in the field'.

\subsubsection{Boasson's model based model}

Boasson [15] defines a 'model based' model that describes the assessment and reactions in a Command and Control system. It is interesting because here the assessment task is explicitly mentioned. The model consists of the following parts: 1) assess, 2) decision, 3) support and 4) control. The function assess refers to situation assessment. The function decision refers to planning of the own forces'. The function control refers to the execution of the operational plans. All these functions are supported by human machine interfaces and models. This model has a broader scope than the JDL model but it lacks any formalization and detailed specification of functions and models.

\subsubsection{The MATIS architecture}

An other interesting Command and Control model is the French Matis architecture (Modèle d'Aide à la décision par le Traitement d'Information Symbolique) developed at the DRED (Direction de la Récherche et des Etudes 


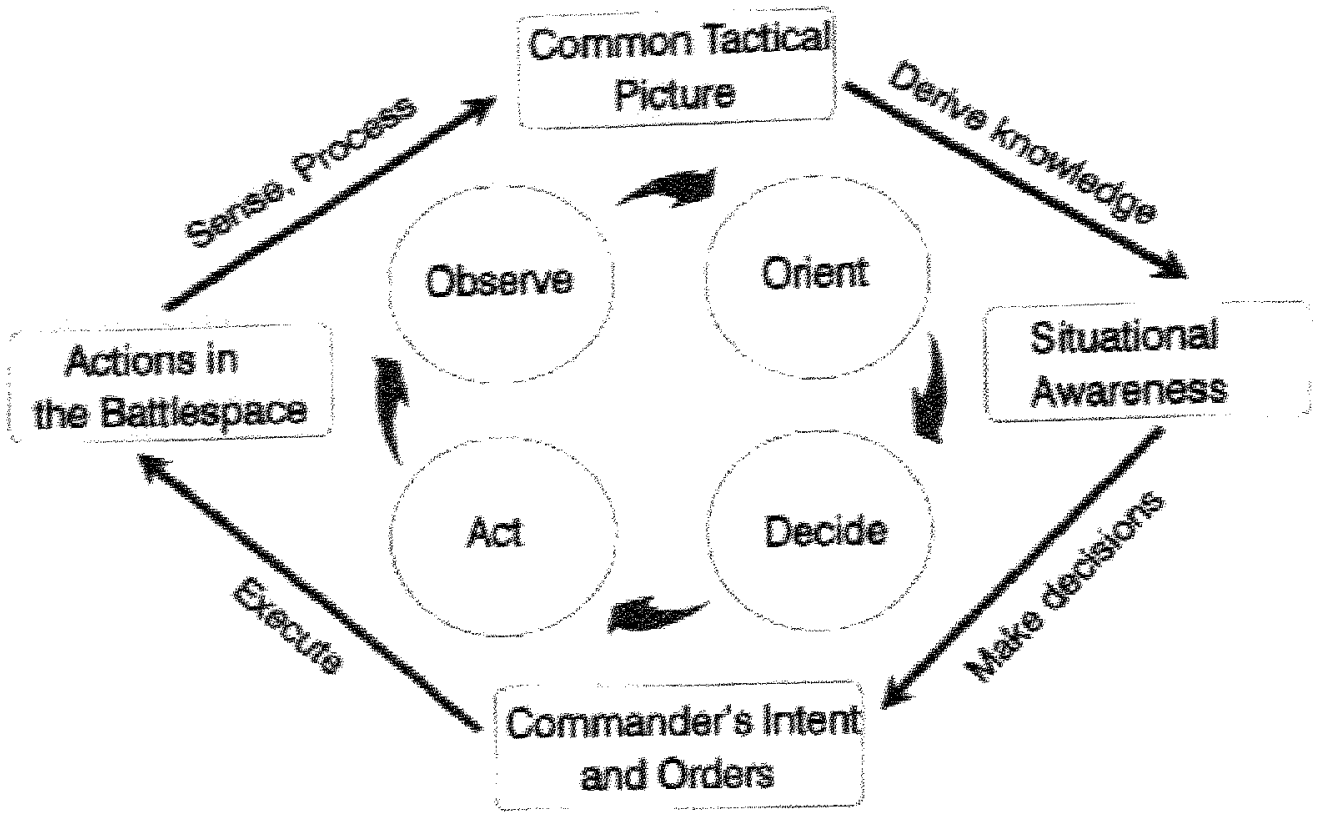

Figure 2.2: Boyl's OODA loop, from 'Process of Naval Command and Control 26 .

Techniques). It is described by Barès in his 1996 book [9] and it is mentioned in Cañamero [20, 21]. Matis poses a Generic Task Model (which is similar to that of Boasson), but goes further by attempting to find a unified data and knowledge representation for the military world. However, it does not define a unified theory for inferences with the military data entered into the system. In the book of Barès, detailed models of Matis are presented and also attempts at formal descriptions of the situation assessment's tasks.

\subsubsection{A plan oriented model}

The Generic Task Model of Mulder, $[56,65,66]$, focusses on planning and plan recognition in a Command and Control system and it is given in figure 2.4. It has two main components: enemy plan recognition and own force (reactive) planning. The Command and Control system interfaces 


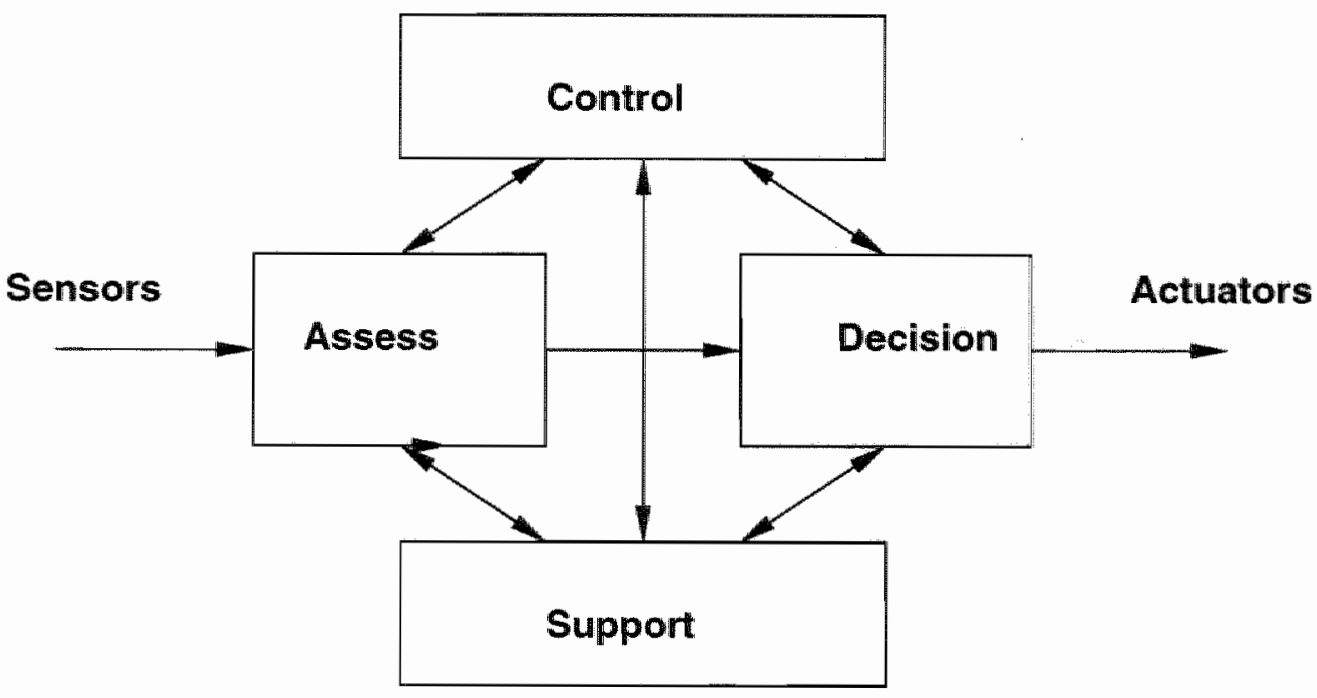

Figure 2.3: Boasson's model of a Command and Control system from [15]

with its boundary, the military world. The system attempts to understand a tactical situation, which it perceives from its sensors and from intelligence messages about enemy forces' objects. It understands the tactical situation by deriving hypothetical enemy forces' plans that explain the enemy forces' observed actions and observed states. Then, based on these hypothetical enemy forces' plans, own forces' plans are devised in- or pre-operation. So during the execution of the operational plan or before the execution.

Both tasks are supported by two generic tasks: model maintenance and determine mission. Model maintenance keeps the models of the outside world up-to date. Determine mission discovers hypothetical enemy missions (goals and constraints) during plan recognition and aids in developing own forces' missions during own forces' planning. For each hypothetical plan one can, pre-operation (before executing the own forces' plan), compose own forces' plans which counter the expected enemy forces' plans and also achieve the own forces' goals. Own forces' planning can also be done in-operation, so during the execution of an own forces' plan this plan is adapted to the actual situation. Thus, own forces' planning is an instance of the problem class reactive planning.

Both tasks plan recognition and model maintenance constitute the higher 


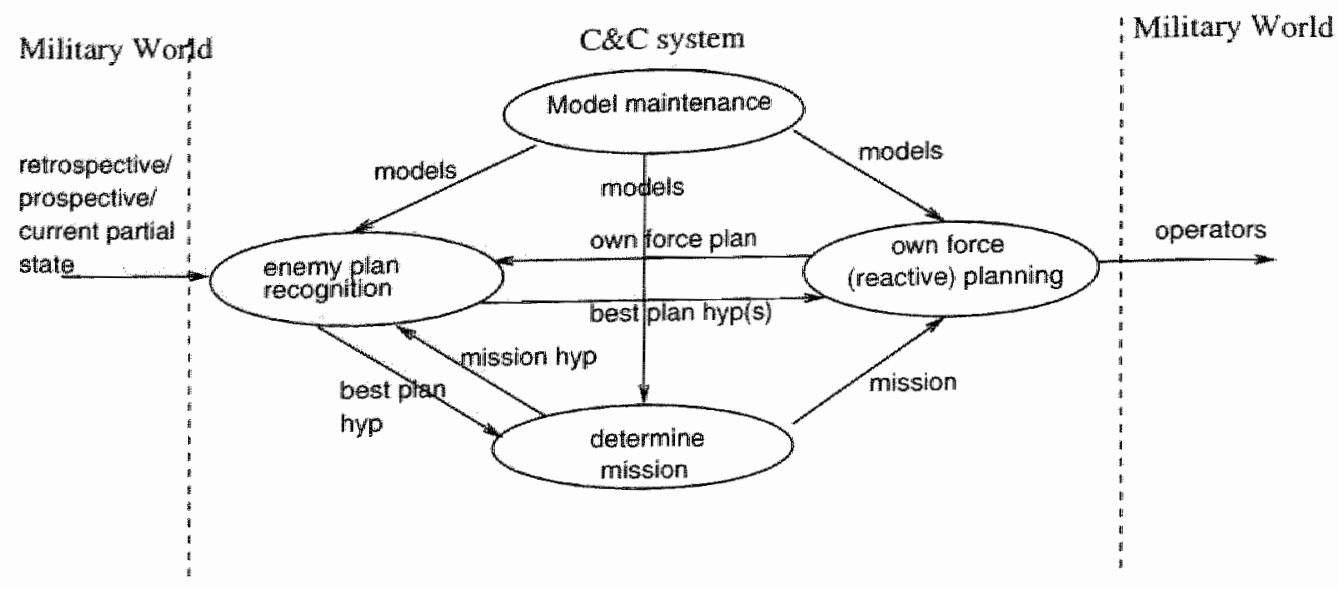

Figure 2.4: Planning and plan recognition in a military Command \& Control System (from Mulder $[56,65,66]$ ).

level situation assessment task. The tasks determine mission and own forces' (reactive) planning constitute the higher level control task. A modern view on obtaining situation awareness, that is aided by the situation assessment function, is described in Houtsma [39]. Here detailed descriptions of tracking, identification and plan recognition functions are given and the functions are related to each other in the paper. Indeed this is the first Command and Control model in which plan recognition is identified as a separate function, atter the paper of Mulder [56].

By means of the Generic Task Model, presented in this chapter, we have some insight in the workings of Command and Control systems. In the remainder of the thesis, we will focus on the Tactical Plan Recognition task. In the next chapter, three examples of Tactical Plan Recognition problems will be given. The examples will clearly show the characteristics of Tactical Plan Recognition.

\subsection{Examples of Tactical Plan Recognition}

In this section two example situations from Mulder and Voorbraak [65], and an additional air force example will be presented. The examples are rather simple, but allow examination of the Tactical Plan Recognition process as far as the scope of this thesis. Regardless of their simplicity, they have some 
'educational' value and have a paradigmatic character. They clearly show the uncertainty about the observations' object's identities and how to handle this uncertainty during plan recognition.

The first example is positioned in the world of civil aviation and can be classified as a 'toy' problem. The second example still is very simply, but shows how a plan recognizer can fail by making wrong identifications. Here we introduce the notion of assignment hypothesis to address the problem of making wrong identifications of observed objects. The last example is from the army military world and is more realistic. Here a well known graphical representation of assignment hypotheses is introduced: the hypothesis tree.

\subsubsection{Civil aviation example}

Though Tactical Plan Recognition is a real problem in the military world, here a simpler 'toy' problem from the world of civil aviation is presented. It will be applied to the examination of formal properties of plan recognizers in this thesis (this work is found in part II). The formal properties are then used for checking algorithms for Tactical Plan Recognition presented in this report. The civil aviation example concerns flights between London and Frankfurt and visa versa.

Example 2.1 The civil aviation example. Consider two airplanes S("Swaen") and Z ("Zwaluw") that can be used for two routes: KL174 from H (Heathrow) to $F$ (Frankfurt), and KL175 from Frankfurt to Heathrow. Both flights stop in A (Amsterdam). This results in four plans: Flight(KL174, S), Flight(KL174, Z), Flight(KL175, S), and Flight(KL175, Z). The background knowledge $\Gamma$ contains the following first order logical formulas: $\forall x$ (Flight $(K \operatorname{Li74}, x) \Leftrightarrow$ $\operatorname{Fly}(\mathrm{H}, \mathrm{A}, x) \wedge \operatorname{Stop}(\mathrm{A}, x) \wedge \mathrm{Fly}(\mathrm{A}, \mathrm{F}, x))$ and $\forall x(\mathrm{Flight}(\mathrm{KL} 175, x) \Leftrightarrow \mathrm{Fly}(\mathrm{F}$, $A, x) \wedge \operatorname{Stop}(A, x) \wedge F l y(A, H, x))$. The logical equivalences link the plan, Fight, with a number of plan parts: Fly, Stop, Fly. For simplicity, there are no temporal considerations. The flights are represented as conjunctions of actions.

In a more advanced plan template, the sequence of actions is also modelled. By example, $[F l y(H, A, S), S t o p(H, A, S)]$. Now the sequence of the observations (defined by the observations' time) must match the specified sequence of the total order, e.g. $[S t o p(A, S), F l y(H, A, S)]$ will not fit any flightplan. 


\subsubsection{The air force example}

In this section a tactical situation will be introduced and its development in a number of stages will be shown. The geography of is given in figure 2.5 .

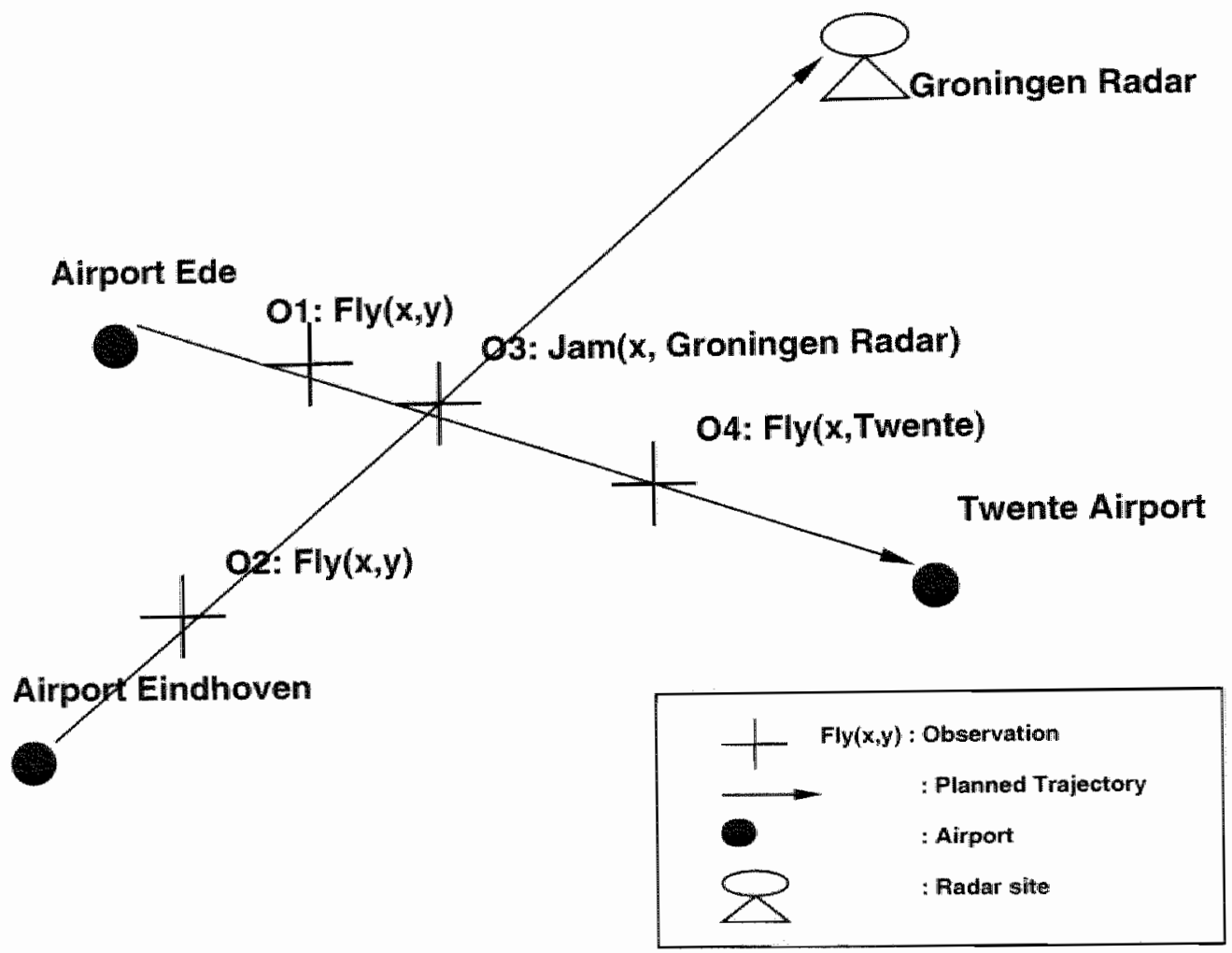

Figure 2.5: A geographical map of the example air force tactical situation.

Example 2.2 The air force example. It concerns three airports and one radar site. In the example, two plans are being executed simultaneonsly by two aircrafts: 1) one aircraft flies from airport Ede to Twente airport to photograph the installations at Twente airport (logically stated as: Photo(A1, Twente), here $A 1$ is an aircraft), and 2) another aircraft flies from Eindhoven airport in the direction of a radar at Groningen, with the intention of jamming ${ }^{1}$ the functioning of the radar ( $\operatorname{Jam}(A 2$, Groningen $)$ ). There are two observations

\footnotetext{
"Jamming is to electronically disturb the correct function of a sensor
} 
of the Ede aircraft fyying to Twente, and two observations of the Eindhoven aircraft. The latter aircraft is first observed flying and is later observed jamming the Groningen radar. The observations and the observed positions of the targets (indicated by crosses) are displayed in figure 2.5. The sequence: $[01,02,03,04]$ is the input of a plan recognizer.

Now let us consider the hypothetical plans that can be deduced from the observations of figure 2.5. From this simple example it is quite clear that the jamming observation is easily, but wrongly, attributed to the Ede aircraft by a plan recognizer. This because the position of the observation is near the first observation of that aircraft. Consequently one assumes that the Ede aircraft is jamming the radar and will fly towards Groningen. Worst case, the second Fly ( $x$, Twente) observation will then be attributed to the Eindhoven aircraft which is hypothesized willing to photograph Twente airport. So now the plan recognizer has two wrong plan hypotheses and has not functioned correctly!

The fault originates in the wrong and pre-mature correlation of observations that are thought to originate from the same object. Some systems therefore consider the correlations to be hypothetical and retract a correlation if a better one becomes available. Yet this is not yet a complete description. In this research we aim a a complete description of these correlation hypotheses by stating observation-plan hypothesis assignment hypotheses. An assignment is a binary relation between the set of observations and the set of plan hypotheses. The assignment hypotheses are stated in table 2.1.

Clearly the number of hypotheses increases significantly as more observations are entered into the plan recognizer. Yet the set of assignment hypotheses always contains the two correct plan hypotheses:

$$
\operatorname{Jam}(A 2, \text { Groningen }), \text { Photo(A1, Twente). }
$$

\subsubsection{The army example}

In this section an army tactical situation will be introduced and its development in a number of stages will be shown. A sequence of observations of enemy helicopters is entered into the plan recognizer. The plan recognizer tries to state a number of assignment hypotheses rather than plan hypotheses. The assignment hypothesis is a unique relation between the set of the observations and the set of plan hypotheses. An assignment hypothesis is based upon previous assignment hypotheses and a new observation. The as- 
32 CHAPTER 2. TACTICAL PLAN RECOGNITION (TPR) RESEARCH

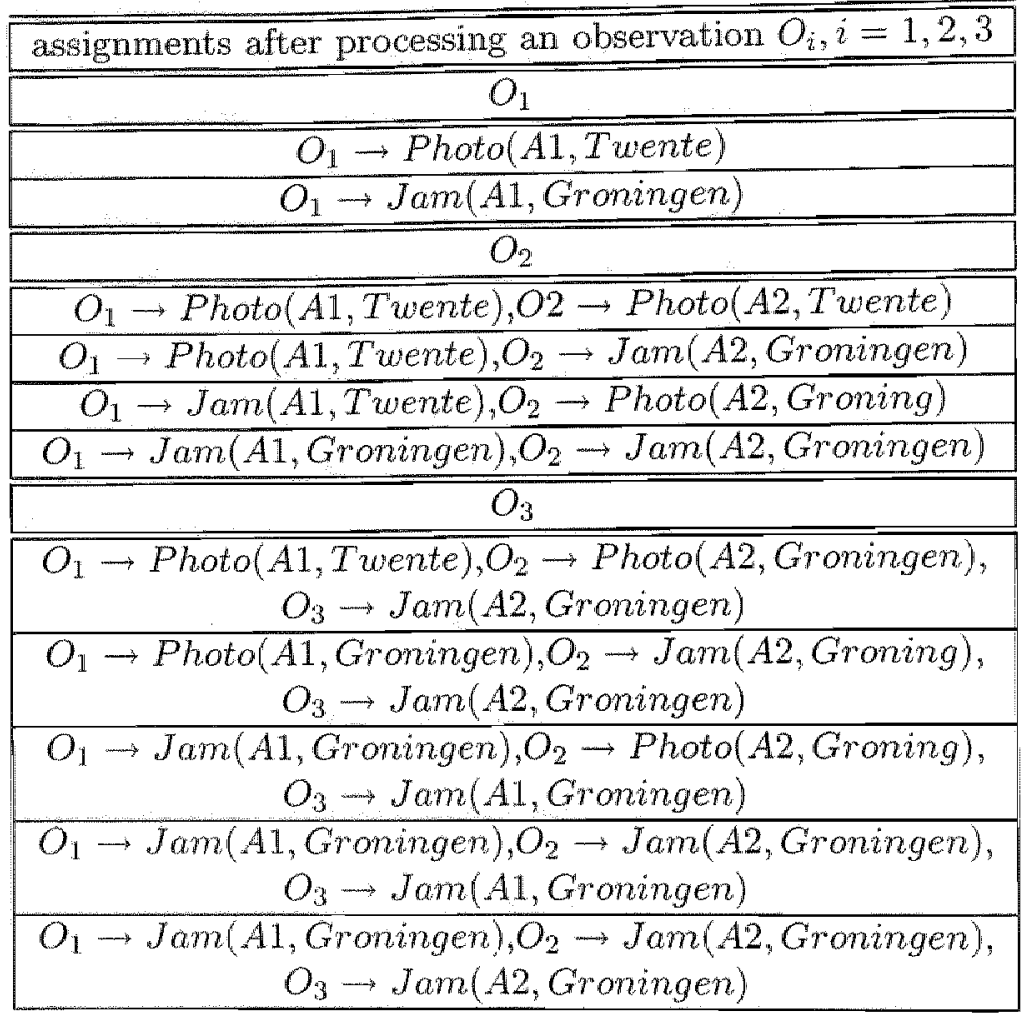

Table 2.1: Assignment hypotheses of the air force example. 
signment hypotheses evolve through time and, after the last observations, the plan recognizer maybe quite certain about the enemy plan.

Example 2.3 The army example. Consider the following example of Tactical Plan Recognition coming from tactical support of ground troops by armed helicopters.

1. In figure 2.6 (page 35) a tactical situation is displayed. The symbols of this figure are given in the legenda, at the bottom of figure 2.6. In the middle of figure 2.6 there is the line representing the front of the battle field, a line dividing the combatants: the red enemy forces and the blue own forces. To the left of the front are the red force units with an air base (indicated by the rectangular icon) as unit $A B 1$, a forward air refuelling point (FARP) below the air base as unit FARP2 (indicated by the circular icon), and close to the front a communication unit, unit COMMS3, (indicated by the triangular icon). To the right of the front are the blue forces consisting of three tank units (units TK4,TK5, TK6) and a munitions depot (indicated by the circular icon) as unit MUN7. Ground support entails support of battle helicopters and aircraft for own forces' ground troops. It concerns attacks on enemy ground troops near or at the front. A ground support plan consists of the following actions: 1) Hy from an air base or a forward refuelling point towards a communication unit, 2) receive target information from the communication unit and 3) ingress, attack and egress to a forward refuelling point or an air base. The task now is to recognize red ground support operations.

2. In the following figure 2.7 (page 36) there is a first observation (O1) of an red armed helicopter. The arrow near the observation indicates the direction of flight. In figure 2.12 (page 44) the possible plan hypotheses are presented in a tree. For the first observation there are two plan hypotheses: 1) the helicopter of $\mathrm{O} 1, \mathrm{H1}$, attacks the munitions depot: A(H1,MUN), the plan's trajectory is indicated by the solid arrow, 2) H1 attacks the tank unit TK5: $\mathrm{A}(\mathrm{H} 1, \mathrm{TK} 5)$, this trajectory is indicated by the dashed arrow. Other possible targets than TK5 and MUN are not considered for simplicity of the example. The plan hypotheses are displayed in the assignment hypothesis tree of figure 2.12 (page 44) above O1. The assignment hypotheses correspond with the branches of the tree. 
3. In figure 2.8 (page 37) a second observation (O2) enters the plan recognition system. Now there are various hypotheses with regard to the origin of the observation. It might be $\mathrm{H} 1$ of the first observation $\mathrm{O} 1$ or it might be a second helicopter $\mathrm{H}_{2}$. The helicopter of the second observation will only attack the tank unit 5: A(H2, TK5). Three assignment hypotheses can occur: 1) in figure 2.8 , page 37 , the first assignment hypothesis is presented: $A(H 1, M U N), A(H 2, T K 5), 2)$ in figure 2.9 (page 38) the arrows represent the plan hypotheses of the second assignment hypothesis: $\mathrm{A}(\mathrm{H} 1, \mathrm{TK} 5), \mathrm{A}(\mathrm{H} 2, \mathrm{TK} 5), 3)$ finally when the observations both originate from $\mathrm{H} 1$ : $\mathrm{A}(\mathrm{H} 1, \mathrm{TK} 5)$, the figure 2.10 (page 39). These hypotheses can also be found in the tree of figure 2.12 (page 44) above $\mathrm{O} 2$.

4. In figure 2.11 (page 40) a final third observation is entered. The observation matches very well with the plan of $\mathrm{H} 1$ attacking tank unit 5 . At this stage there is very little support for the other hypotheses that are possible.

This concludes the example.

Again, consider the hypothesis tree of figure 2.12 (page 44). Above each observation there are branches that are labelled with the hypothetical plan for the objects of the oloservation. The leafs of the tree state the assignment hypothesis after three observations. An assignment hypothesis combines the individual plan hypotheses at the branches. However, from the assignment hypotheses, only the plans are listed in this figure, e.g. $\mathrm{A}(\mathrm{H} 1, \mathrm{MUN}), \mathrm{A}(\mathrm{H} 2, \mathrm{TK} 5)$. A more elaborate specification of the assignment hypotheses is found in table 2.2 .

In table 2.2 the assignment hypotheses, that can occur when observations enter into the system, are given. The table consists of three sets of rows, one set for each observation, i.e. O1 till O3. For each set, all assignment hypotheses are displayed. For example for $\mathrm{O} 2$, the assignment hypotheses are $\Psi_{2,1}, \Psi_{2,2}$ and $\Psi_{2,3}$. In the right column all the elements of the assignment hypotheses are displayed. For example $\Psi_{2,1}=\{\mathrm{O} 1 \rightarrow \mathrm{A}(\mathrm{H} 1, \mathrm{MUN}), \mathrm{O} 2 \rightarrow$ A $(\mathbb{H} 2$, TK5 5$)\}$.

Now the example is finished, we will continue with the examination of related work in the next chapter. 


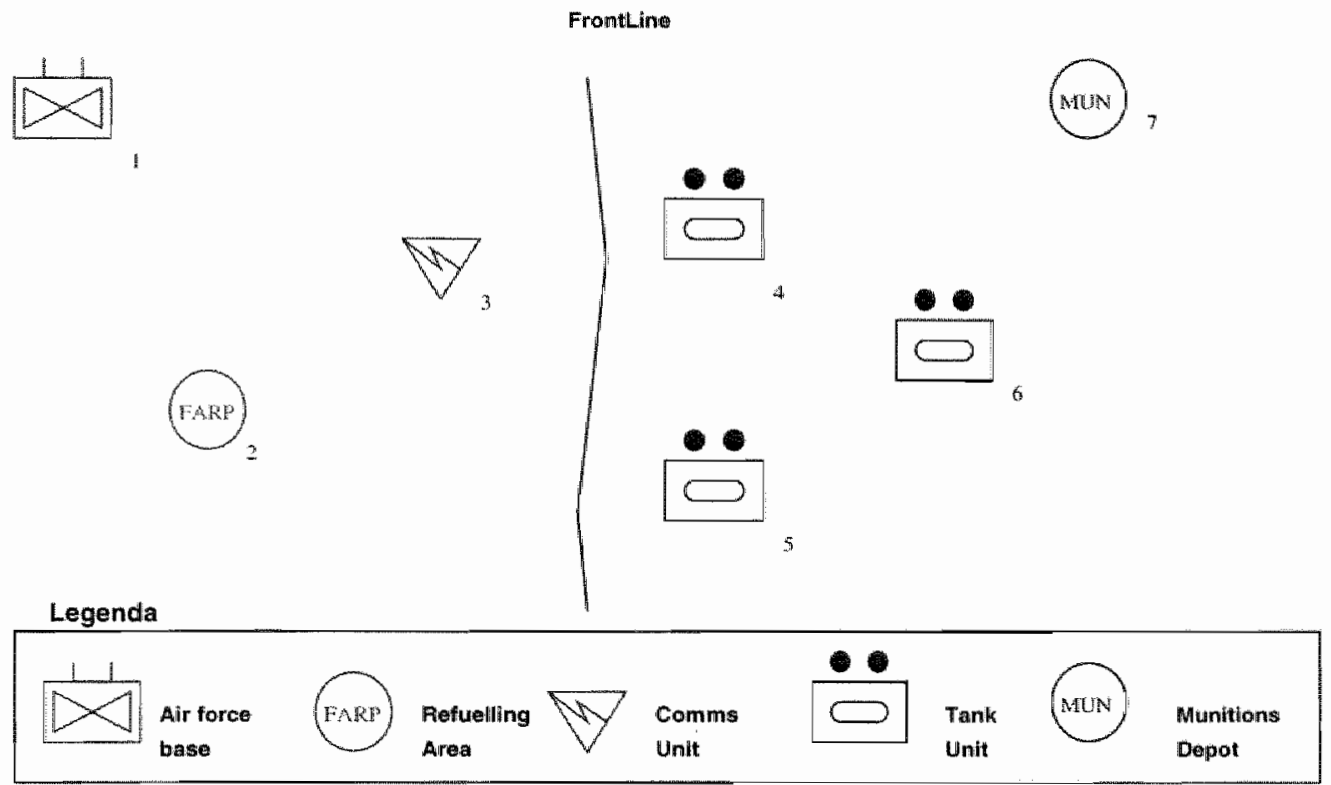

Figure 2.6: A geographical map of the tactical situation of the army example. The position of the friendly units (on the right of the front line) and the enemy units (on the right side of the front line). in the army example The legenda of the military style icons is given in the rectangle at the bottom of the figure.

\subsection{Tactical Plan Recognition research}

In this section research work in the area of Tactical Plan Recognition will be discussed. The first work on automating plan recognition was done by Azarewicz et. al. His work and his successors are first mentioned. Then we will focus on other systems that only handle identified observations. In the next section, we will focus on plan recognizers that are more realistic and can handle unidentified observations. The final section concerns systems that focus on learning doctrines, rather than the plan recognition process itself.

\subsubsection{The first automated Tactical Plan Recognition system}

As mentioned before, the first work on automating plan recognition was done by Azarewicz et. al. In his publications in 1986 and 1988, [7, 8], Azarewicz developed, a rather complex, generic goal/plan descriptions that describe enemy 

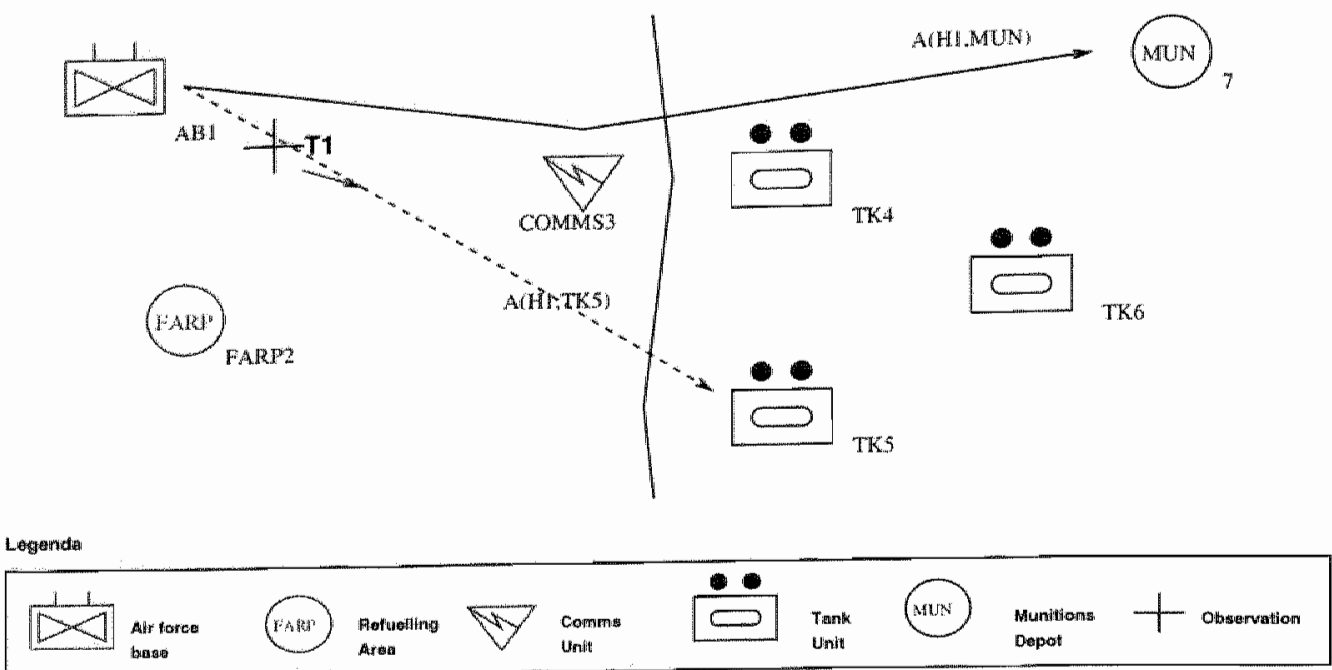

Figure 2.7: A geographical map of the tactical situation of the army example. Plan hypotheses after the first observation of Helicopter H1. The two plan hypotheses are: 1) the Attack(H1,TK5) (solid arrow, it corresponds with assignment hypothesis $\Psi_{1,1}$ of table 2.2) and 2) Attack(H1,MUN) hypotheses (dashed arrow, it corresponds with $\Psi_{1,2}$ ).

doctrines. This approach of development of complex data structures being erected and modified by expert systems was often found in Artificial Intelligence research at that time. As an illustration, examples of Azerewicz's data structures are given in figures 2.13 (page 45), 2.14 (page 46) and 2.15 (page 47). When receiving the first observations, the generic descriptions are instantiated to a set of active goal/plan hierarchies. His hierarchy consists of top goals and plans at the highest hierarchical level. An example of this is given in figure 2.13. At the top one finds possible goals of the observed object. It concerns three goals of a military 'attack target' mission: reconnaissance of enemy targets, the destruction of a target and the defence against the target's counter measures. The top goals are linked to multi-agent plan templates and, at the bottom, one finds single-agent plan templates. Single-agent plans are a part of the multi-agent plans. The multi-agent plans describe roles of agents (e.g. tanking, coordination, counter measures). Azarewicz then compiles a set of hypothetical agent-role matrices in which the level of matching of observed agents to roles of the instantiated plan is described. An example of a multi- 


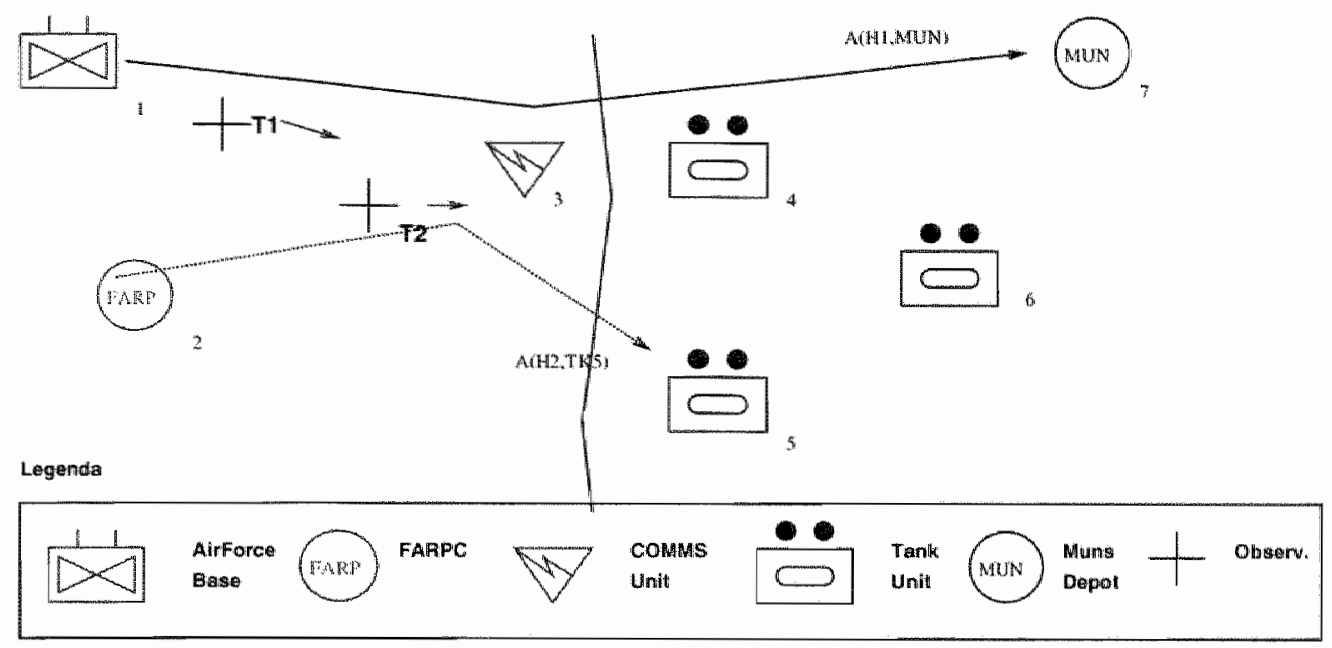

Figure 2.8: A geographical map of the tactical situation of the army example. An assignment hypothesis ( $\Psi_{2,1}$ of table 2.2$)$ after two observations: one observation of Helicopter H1 and one of helicopter H2. The depicted assignment hypothesis consists of the following two plan hypotheses: 1) one plan hypotheses (upper solid arrow) concerns the attack of $\mathrm{Hl}$ on the munitions depot MUN, 2) the other plan hypotheses concerns the attack of another helicopter $\mathrm{H} 2$ on the tank unit TK5 (lower dashed arrow).

agent plan is given in figure 2.14. The multi-agent plan consists of a temporal template and a spatial template. Each multi-agent plan has a pre condition, that must be true before plan execution and a post condition that is true after execution of the multi-agent plan (pre- and post conditions are also found in STRIPS operators). At the lowest level in the hierarchy are single-agent plans positioned. This is modelled by a Deterministic Finite Automaton. An example of a single-agent plan is given in figure 2.15. Here the Ingress concerns fying towards the target, On-station concerns the attack of the target and, finally, the Egress concerns flying to the home base. The following observations are used bottom up to determine the support for observable conditions for various agent roles in the agent-role matrix.

Like much work of that time, it is based on psychological models and applies heuristics to define a measure of belief (MB) for its plan hypotheses. Azarewicz' work focusses on the operations on its goal/plan descriptions. The input are identified observations. 


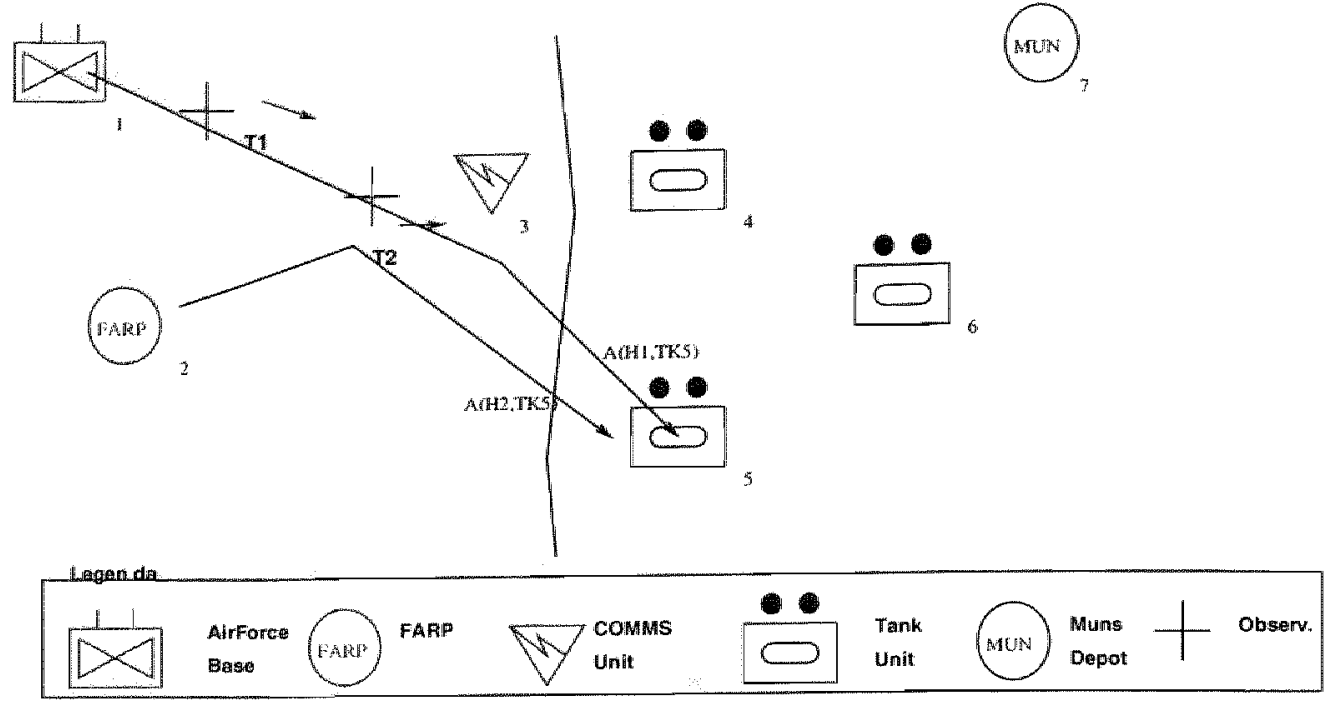

Figure 2.9: A geographical map of the tactical situation of the army example. The second assignment hypotheses ( $\Psi_{2,2}$ of table 2.2$)$ after two observations. The depicted assignment hypothesis consists of the following two plan hypotheses: 1) one plan hypotheses (upper solid arrow) concerns the attack of H1 on the tank unit TK5, 2) the other plan hypotheses concerns the attack of another helicopter $\mathrm{H} 2$, also on the tank unit TK5 (lower solid arrow).

In the US some research followed that of Azarewitz, e.g. [69] in 1987 and in 1998. However, this research was only presented in US restricted conferences, such as the Tri-Service Data Fusion Symposium.

\subsubsection{Systems for identified observations}

Canamero, [21], proposed a knowledge-level model for Tactical Plan Recognition. In edge from military intelligence officers on the human plan recognition process. Canamero used this to generate a Generic Task Model. It differs on the work of Azarewicz in that this work is based on expert system development methods and results in a Generic Task Model. The input of this system are identified observations.

In work on military distributed simulation (DIS) such as that of Tambe and Rosenbloom, $[85,86]$ applies plan recognition for a software agent. Here 


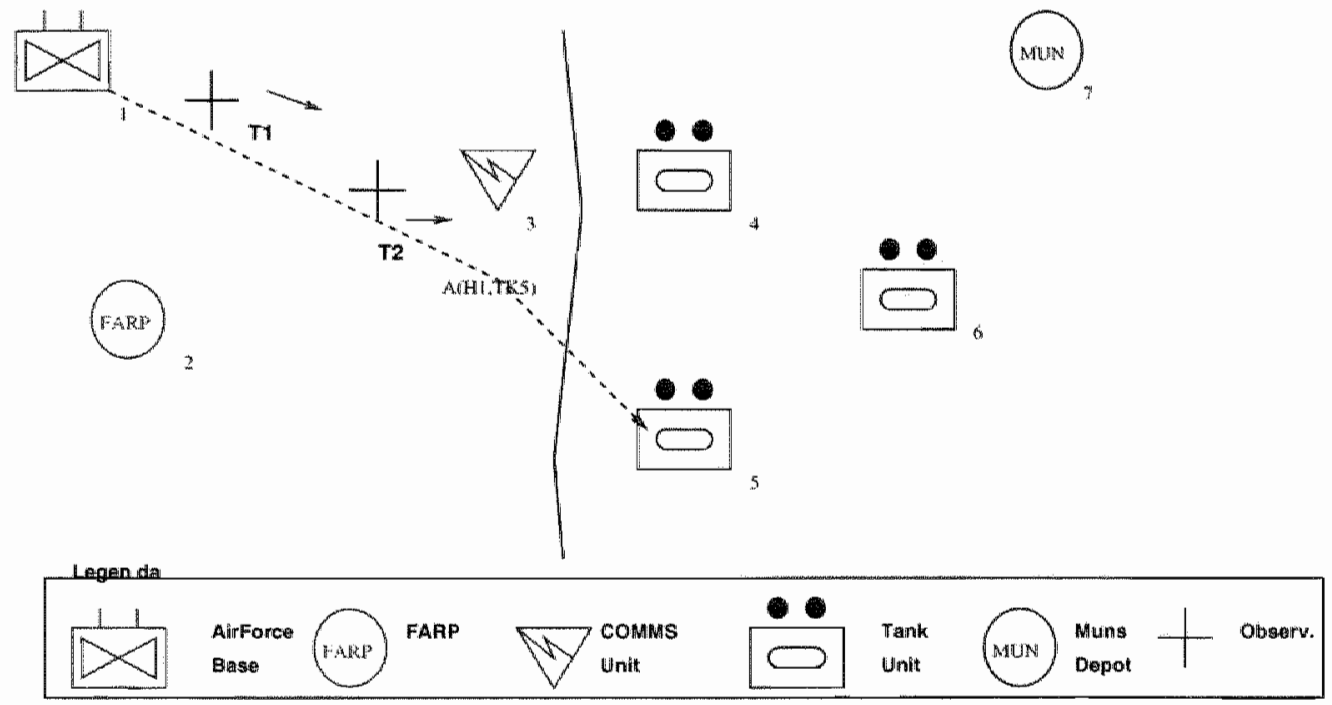

Figure 2.10: A geographical map of the tactical situation of the army example. The third assignment hypotheses ( $\Psi_{2,3}$ of table 2.2 ) after two observations. The depicted assignment hypothesis consists of only one plan hypotheses: the attack of $\mathrm{H} 1$ on the tank unit TK5. This because both observations are attributed to helicopter H1.

actions as well as events (non- intentional) of other agents are tracked. They imput identified observations and match the observation"s actions to a generic sequence of events, i.e. the doctrine. However, Azarewicz already used Deterministic Finite Automata for single-agent doctrines, that are more realistic.

The bayesian net plan recognition method of Charniak and Goldman is also applied to enemy plan recognition in the US [13], but (again) no unrestricted publications of this work is available. However, in a later publication of Suzic $[84,83]$ in 2003 , he also applied this technique for reasoning under uncertainty to Tactical Plan Recognition.

\subsubsection{Systems for unidentified observations}

The first system in which unidentified observations where entered into the plan recognizer was the NATO Data Fusion Demonstrator project (DFD). In DFD , from 1995 on, it was attempted to do army Tactical Plan Recognition, though this was not realized by its designers. The most interesting result 


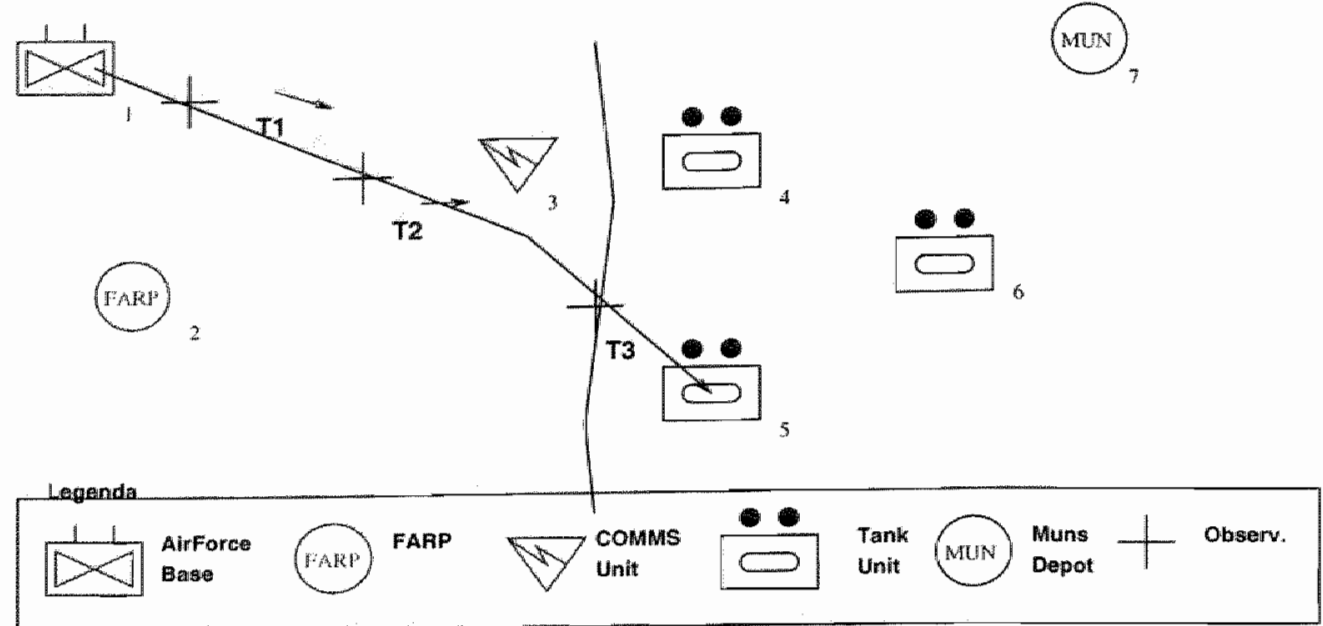

Figure 2.11: A geographical map of the tactical situation of the army example. The processing of the last observation, all three observations are assumed to originate from one helicopter: $\mathrm{H} 1$, The plan hypothesis is found in assignment hypothesis $\Psi_{3,3}$ of table 2.2 .

from DFD is a concise set of models of army doctrines, that can be found in Wols, $[99,98]$. The DFD software, inputs unidentified observations and first attempts to correlate observations in order to recognize subsets of these observations that are thought to originate from the same object. For this a data structure is constructed in a blackboard system ${ }^{2}$. An example of the erected data structure is given in figure 2.16. Initially there are three levels of objects: the observations at the lowest level, the hypothetical targets based on the observations at the middle level and finally the plan hypothesis of a hypothetical target. In the figure there is an ambiguity about where to assign observation $\mathrm{O} 2$.

Thus, the observations are identified and, only then, are input to a traditional identified observation plan recognizer. Then it calculates the support of such a subset for the (by the plan recognizer) generated plan hypotheses for the set's object. The system allows for correlations to be tentative and to be retracted if they lacked support by new observations.

\footnotetext{
${ }^{2}$ A blackboard system consists of a (possibly distributed) global database on which severa] expert systems operate. The expert systems communicate with each other by means of the global blackboard
} 


\begin{tabular}{|c|c|}
\hline assignment & elements of assignment \\
\hline \multicolumn{2}{|r|}{$O_{1}$} \\
\hline$\Psi_{1,1}$ & $\mathrm{OI} \rightarrow \mathrm{A}(\mathrm{H} 1, \mathrm{MUN})$ \\
\hline$\Psi_{1,2}$ & $\mathrm{O} 1 \rightarrow \mathrm{A}(\mathrm{H} 1, \mathrm{TK} 5)$ \\
\hline \multicolumn{2}{|r|}{$\mathrm{O}_{2}$} \\
\hline$\Psi_{2,1}$ & $\mathrm{O} 1 \rightarrow \mathrm{A}(\mathrm{H} 1, \mathrm{MUN}), \mathrm{O} 2 \rightarrow \mathrm{A}(\mathrm{H} 2, \mathrm{TK} 5)$ \\
\hline$\Psi_{2,2}$ & $\mathrm{O} 1 \rightarrow \mathrm{A}(\mathrm{H} 1, \mathrm{TK} 5), \mathrm{O} 2 \rightarrow \mathrm{A}(\mathrm{H} 2, \mathrm{TK} 5)$ \\
\hline$\Psi_{2,3}$ & $\mathrm{O} 1 \rightarrow \mathrm{A}(\mathrm{H} 1, \mathrm{TK} 5)$ \\
\hline \multicolumn{2}{|r|}{$O_{3}$} \\
\hline$\Psi_{3,1}$ & $\mathrm{O} 1 \rightarrow \mathrm{A}(\mathrm{H} 1, \mathrm{MUN}), \mathrm{O} 2 \rightarrow \mathrm{A}(\mathrm{H} 2, \mathrm{TK} 5), \mathrm{O} 3 \rightarrow \mathrm{A}(\mathrm{H} 3, \mathrm{TK} 5)$ \\
\hline$\Psi_{3,2}$ & $\mathrm{O} 1 \rightarrow \mathrm{A}(\mathrm{H} 1, \mathrm{MUN}), \mathrm{O} 2 \rightarrow \mathrm{A}(\mathrm{H} 2, \mathrm{TK} 5)$ \\
\hline$\Psi_{3,3}$ & $\mathrm{O} 1 \rightarrow \mathrm{A}(\mathrm{H} 1, \mathrm{TK} 5), \mathrm{O} 2 \rightarrow \mathrm{A}(\mathrm{H} 2, \mathrm{TK} 5), \mathrm{O} 3 \rightarrow \mathrm{A}(\mathrm{H} 3, \mathrm{TK} 5)$ \\
\hline$\Psi_{3,4}$ & $\mathrm{O} 1 \rightarrow \mathrm{A}(\mathrm{H} 1, \mathrm{TK} 5), \mathrm{O} 2 \rightarrow \mathrm{A}(\mathrm{H} 2, \mathrm{TK} 5)$ \\
\hline$\Psi_{3,5}$ & $\mathrm{O} 1 \rightarrow \mathrm{A}(\mathrm{H} 1, \mathrm{TK} 5), \mathrm{O} 2 \rightarrow \mathrm{A}(\mathrm{H} 2, \mathrm{TK} 5)$ \\
\hline$\Psi_{3,6}$ & $\mathrm{O} 1 \rightarrow \mathrm{A}(\mathrm{H} 1, \mathrm{TK} 5), \mathrm{O} 3 \rightarrow \mathrm{A}(\mathrm{H} 3, \mathrm{TK} 5)$ \\
\hline$\Psi_{3,7}$ & $\mathrm{O} 1 \rightarrow \mathrm{A}(\mathrm{H} 1, \mathrm{TK} 5)$ \\
\hline
\end{tabular}

Table 2.2: Assignments in the army example.

Given a set of observations, all its combinations of subsets are found in the power set. However this has an exponential complexity. A practical approach for this correlation problem is the 'pair-wise' combination technique that was actually used. It is a heuristic technique for sensor data fusion introduced by Miles [34] in 1988.

However, the observation correlator has no knowledge of plan hypotheses nor does it account for a new plan hypothesis for each new observation. The correlator cannot use a hypothetical plan for prediction of a future state or future action of an object or a number of objects. Thus, it has insufficient information to do medium term predictions (e.g. several minutes to several hours). Also a mayor problem with the DFD project was the inadeguate software design and, in fact, the system never worked properly!

In 1998 Mulder [56] recognized the similarity of Tactical Plan Recognition to Multiple Hypothesis Tracking from Reid [74] and proposed to consider observation-plan hypothesis assignment hypotheses. As shown in the examples, these assignment hypotheses generate all possible observation-plan correlations that are possible. Reid's formula then gives a likelihood estimation of the probability of each assignment hypothesis. By this probability, the hy. 
potheses can be ranked. The correlation probability, that is an element of Reid's formula, is estimated by the likelihood of a statistical distance between the observation and the plan hypothesis. The estimation applies a stochastic model of kinematic variables of the observed target.

Our work is similar to that of Mevassvik [52] . Here the input are unidentified observations (intelligence reports), tracks and an actual set of flight/sail plans in an area. The plan recognizer then associates these observations to the set of flight and sail plans. The likelihood of an association is defined by a statistical distance measure between observations and plans. In the association they also considers various constraints. Though the authors do input unidentified observations in their system, the plans of the observed objects are known on forehand. In the case of Tactical Plan Recognition the enemy plans are unknown and plan hypotheses have to be created and maintained for each observation.

Interesting is that they apply the mean reverting process from financial modelling as the stochastic model for the kinematic state. In this thesis will be shown that the speed estimation with Mevassvik's model is inferior to a linear constant motion model.

\subsubsection{Systems for learning doctrines}

Some authors focus on learning generic plan descriptions. The focus of this work is entirely different from the other systems, because here the generic plan descriptions are hand-crafted.

Ross in 2000 [75] used Hidden Markov Models of transitions in a DFA plan template, to extract the course of action of a number of units from their spatial order. Ross only attempts to match an observed sequence of actions with possible sequences, according to the Hidden Markov Models. Again the input of this system are identified observations.

Clinton Heinze et.al. [38] focus on learning generic descriptions of spatiotemporal relationships (these are, of course, also present in our military domain) from a set of observations. The relationships define their description of enemy doctrines. They developed the CLARET algorithm that is based on statistical pattern matching learning techniques, that are currently used for on-line handwriting and gesture recognition. 


\subsection{Possible new applications of Tactical Plan Recog- nition}

Tactical Plan Recognition is a problem that finds its origin in the military world. It is a part of a Command and Control System and these are found in the military, the police and fire department. One might envisage that the methods developed for Tactical Plan Recognition are useful in all areas where one finds multiple phenomena in one world, where the phenomena do not reveal themselves and where they change in time. In the medical world, an application might be diagnosis of multiple diseases in a patient.

Now the research on Tactical Plan Recognition research has been introduced, some shortcomings of related work will be discussed in the next chapter. These shortcomings are addressed in a problem statement that summarizes the goals of this research. 


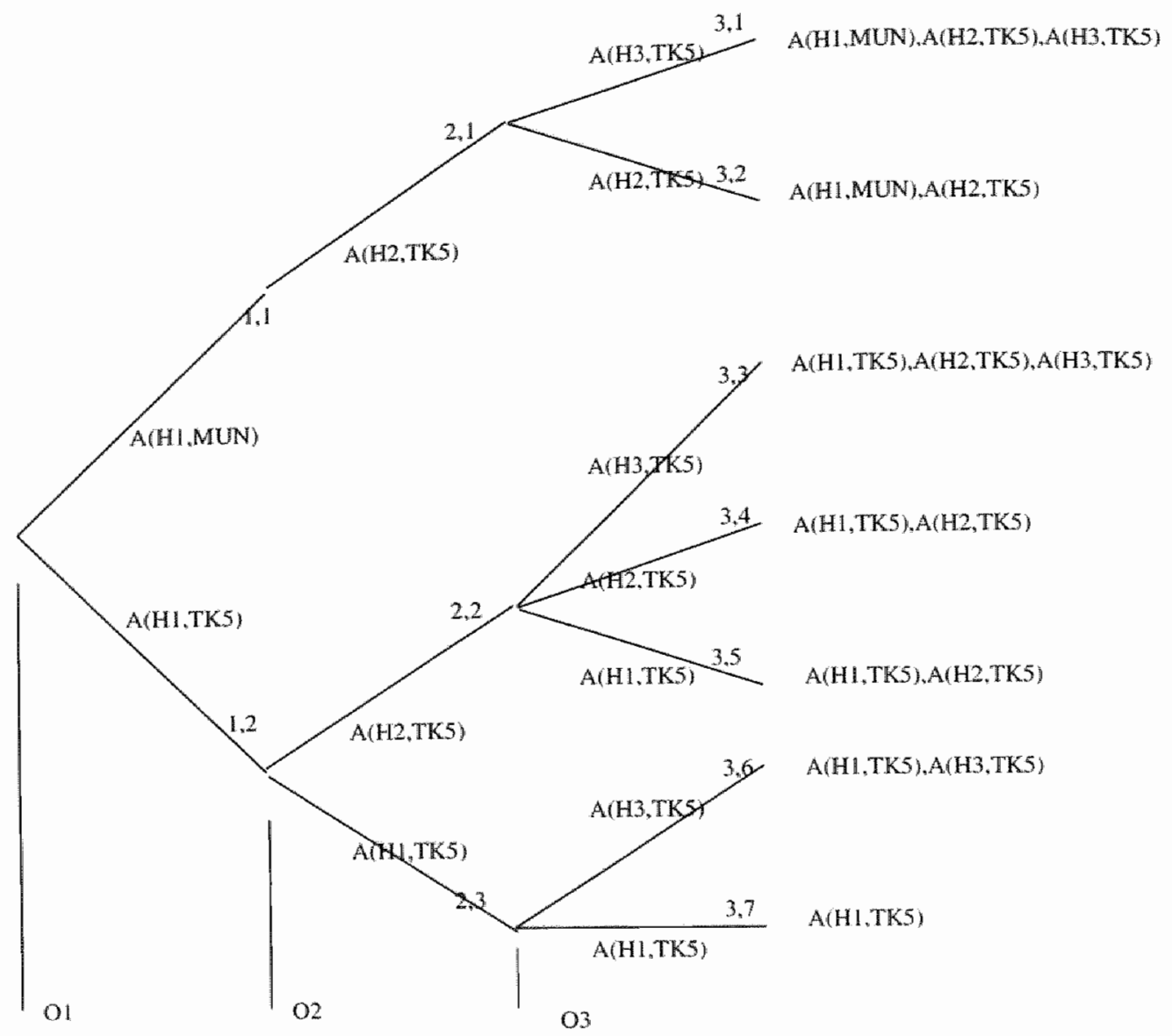

Figure 2.12: The army example: the hypothesis tree. At the bottom of the picture one finds the sequence of observations: $[O 1, O 2, O 3]$. The first observation $O \perp$ forms the root of the tree, the two branches of the root are the two plan hypotheses assigned to $O 1$. Each root branch is further split by plan hypotheses assigned to 02 . But they must exist given the plan hypotheses of the root branch they extend. $O 3$ results in the final branches. 


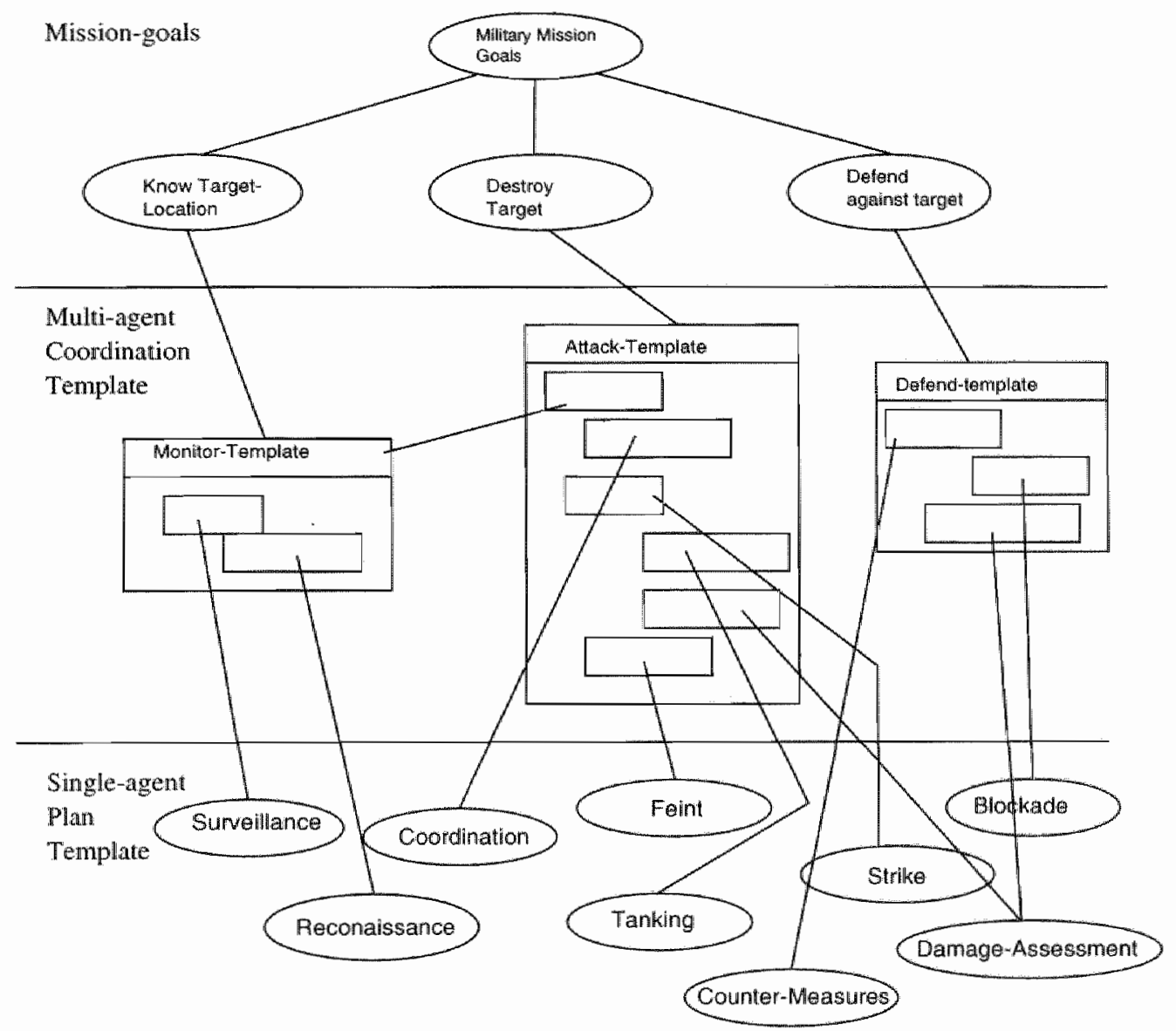

Figure 2.13: An example of Azarewicz's plan hierarchies. 
CHAPTER 2. TACTICAL PLAN RECOGNITION (TPR) RESEARCH

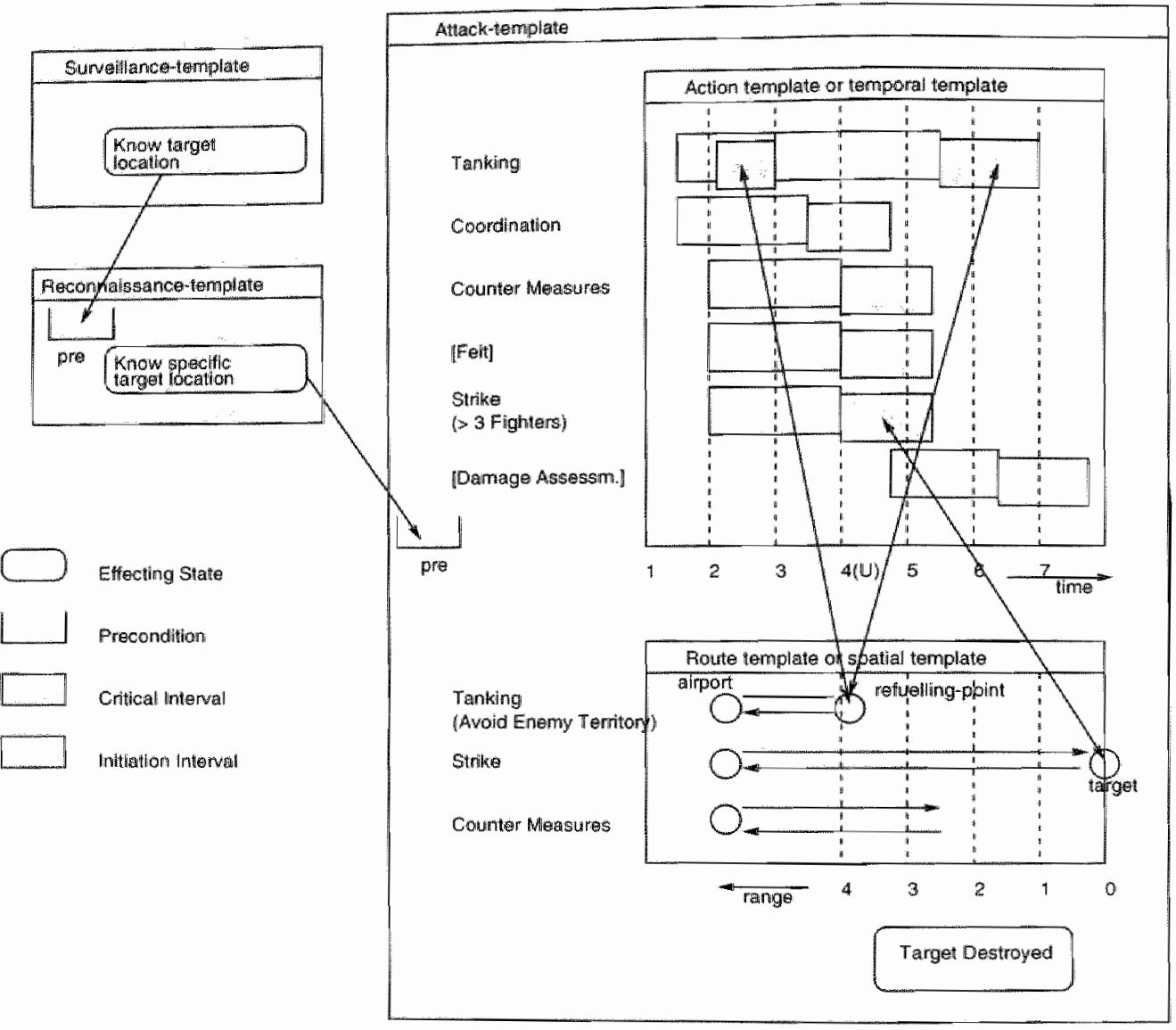

Figure 2.14: An example of Azarewicz's multi-agent plan. 


\section{Ingress sequence On-station Egress}

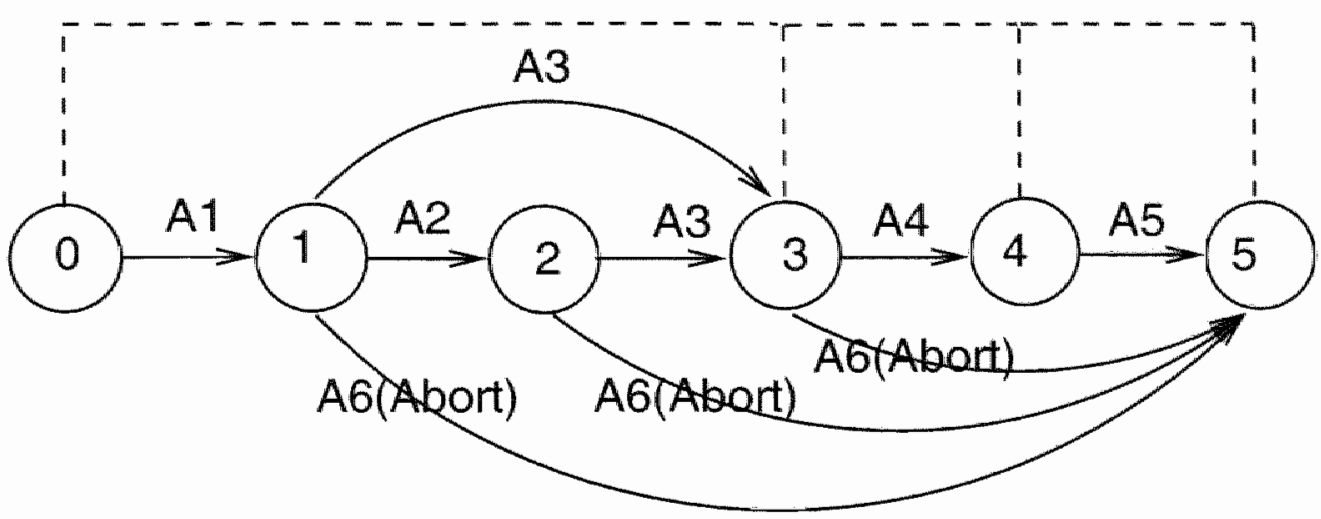

Figure 2.15: An example of Azarewicz's single-agent plan.

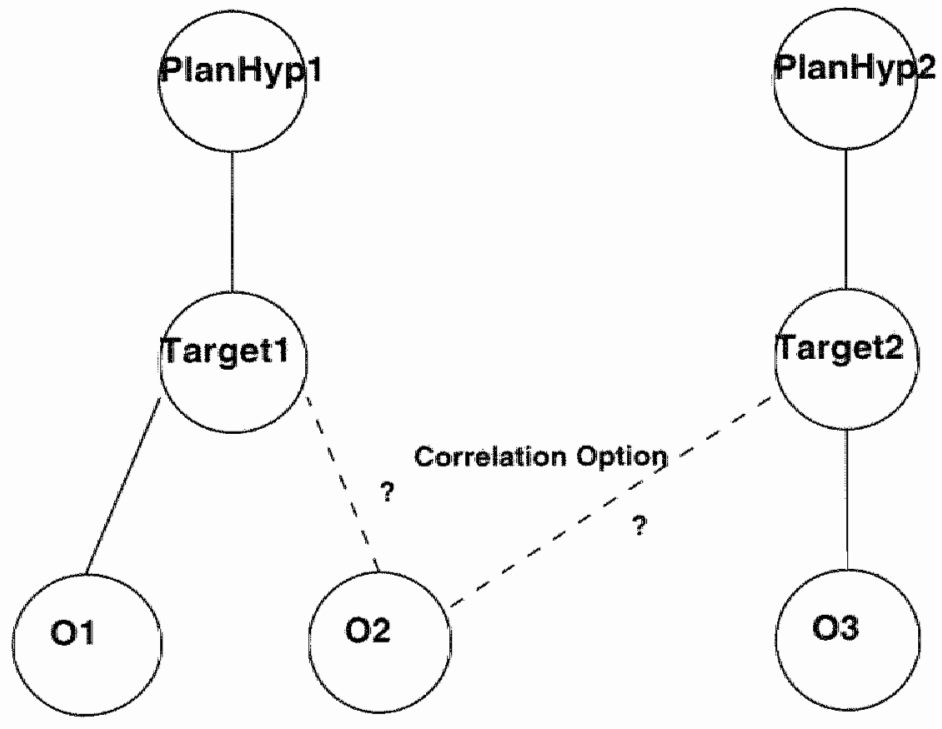

Plan Hypotheses

Hypothethical

Targets

Observations

Figure 2.16: An example of the data structures erected by the DFD system. 
48 CHAPTER 2. TACTICAL PLAN RECOGNITION (TPR) RESEARCH 


\section{Chapter 3}

\section{Problem statement}

In the previous section, the work on Tactical Plan Recognition has been introduced. In this chapter, first the related work on the Tactical Plan Recognition problem is discussed. Some areas for improvement are mentioned here. Then the approach to the Tactical Plan Recognition problem of this work is presented. Our approach consists of two contributions: 1) the application of the Multiple Hypothesis Tracking method to Tactical Plan Recognition and 2) the application of classical abduction for a formal description of Tactical Plan Recognition. The applicability of the contributions of this research is the research question that is found in the problem statement.

\subsection{Discussion of Tactical Plan Recognition research}

In this section first an overview characterizes the most relevant research. Then shortcomings of the current research are summarized as an introduction to the problem statement of this thesis.

\subsubsection{Overview}

First let us give an overview of the work of Azarewicz, the DFD project, Canamero, Mulder and Suzic. In tables 3.1 and 3.2 some characteristics of these systems are given. In table 3.1 the following characteristics are considered: 1) the input observation type, identified or unidentified, 2) the type of the correlator; does the correlator consider correlation hypotheses or not (in the case of a identified observations input; the correlator is not present in the 


\begin{tabular}{|c|c|c|c|c|c|}
\hline system & obs type & con type & hyp type & proc type & $\begin{array}{c}\text { formal } \\
\text { model }\end{array}$ \\
\hline Azerwicz & identified & no & plan & logic, heuristic & no \\
\hline DFD & unidentified & corr hyps & plan & heuristic & no \\
\hline Canamero & identified & no & plan & heuristic & no \\
\hline Mulder & unidentified & corr hyps & ass hyps & statistical & yes \\
\hline Mewassvik & unidentified & corr hyps & ass hyps & statistical & yes \\
\hline Suzic & identified & no & plan hyps & $\begin{array}{c}\text { bayesian } \\
\text { network }\end{array}$ & bayesian \\
\hline
\end{tabular}

Table 3.1: Overview of Tactical Plan Recognition systems.

system), 3) the hypothesis type that is outputted; these are either plan hypotheses or assignment hypotheses, 4) the type of reasoning under uncertainty of the plan recognizer; this can be heuristic, statistical or utilize bayesian nets and the last two characteristic indicate whether some formal method for reasoning under uncertainty has been applied. If the system applies heuristics here, this is not considered a formal method, whereas the statistical methods and bayesian nets are considered to be formall methods.

In table 3.2 the following characteristics are given: 1) whether the method has been shown to be feasible by providing a demonstrator, 2) whether the system's algorithm is sound, 3) whether the system's algorithm is complete, 4) whether there is some comprehensive function of decision variables that is optimized and, finally, 4) whether the system may be useable at all.

The system is useable when a demonstrator is axailable and unidentified observations are the input of the system. The soundness and completeness of an algorithm are described in detail in definitions 5.6 (page 5.6) resp. 5.7 (page 105), but an informal introduction will be given here. The soundness concerns the correctness of the algorithms, the completeness guarantees that a solution will be found if one exists. Clearly these are desirable properties for an algorithm and algorithm developers always will try to proof these qualities for their algorithms.

The system of Azerewicz has a demonstrator so it is certainly feasible. However, as heuristics are applied by him, one cannot ascertain whether the processing is sound or complete. In his paper Azerewicz does not discuss these formal properties. In the function 'task - agent assignment' of the system, an assignment functions is defined and an optimal assignment found. 


\begin{tabular}{|c|c|c|c|c|c|}
\hline system & demonstrator & sound & complete & optimal & feasible \\
\hline \hline Azerwicz & yes & no & no & yes & no \\
\hline DFD & no & no & no & no & no \\
\hline Canamero & yes & no & no & no & no \\
\hline Mulder & no & no & no & yes & no \\
\hline Mevassvik & yes & no & no & yes & yes \\
\hline Suzic & yes & no & no & yes & no \\
\hline
\end{tabular}

Table 3.2: Overview of Tactical Plan Recognition systems.

The DFD system utilizes heuristics and therefore, again, no formal properties can be attributed to it. No form of optimization is applied in the DFD project. As the DFD project's system never functioned properly no demonstrator is available. Because there is no demonstrator the system is classified as not feasible.

The input of Canamero's system are identified observations, so the system is also classified as not feasible. Also Canamero does not discuss any formal properties of her system. She proposes a Generic Task Model, but does not provide for a demonstrator.

Also Mevassvik does not discuss the soundness or completeness of their system, but they state and solve a constrained optimization problem. They have implemented a demonstrator, so their system is certainly feasible. Also Suzic reports of a demonstrator, so also his system is feasible.

The system of Mulder, based upon Multiple Hypotheses Tracking, was not implemented yet at the time of the 1998 publication. However, the 'correct' use of assignment hypotheses promises a sound and complete system. This contrary to systems that do not produce assignment hypotheses. In the system one finds the k-best assignment hypotheses and the correlation likelihood can be calculated by using optimal kinematic state estimation method.s. Therefore the system does perform optimization. Because there is no demonstrator, the system was not, shown to be feasible in 1998 .

\subsubsection{Shortcomings}

The related work only handles identified observations or handles unidentified observations in a sequential manner that is not integrated in the plan recognition process. The only exception is the work of Mevassvik, but his system 
is only suitable for cooperating targets. All the other plan recognizers apply a correlator and then do plan recognition. Disadvantages of this approach are that:

- the correlator cannot apply knowledge that is entailed in the plan hypotheses for correlation.

- Often the plan recognizer does not re-evaluate correlations in later stages.

In the example 2.2 it was shown that sequential Tactical Plan Recognition systems (in which first unidentified observations are correlated to be identified and then are input to a classical identified observation plan recognizer) cannot utilize information available in plan hypotheses in the correlation process. It simple correlates observations only based on the position and other information of the observations themselves. Thus it is not possible to correlate correctly observations that are separated by a long period of time. What is preferred that the observation is directly correlated to the plan hypotheses, and thus all information available is used in the correlation.

Another shortcoming of some other work, is the lack of a comprehensive formal (that is logical and mathematical) model that can be used in their plan recognition algorithms and/or can be used to describe their plan recognizers. Lack of a formal description causes an inability to asses the soundness,

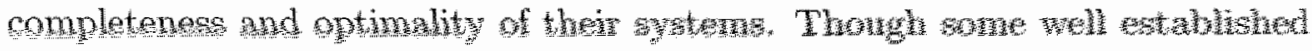

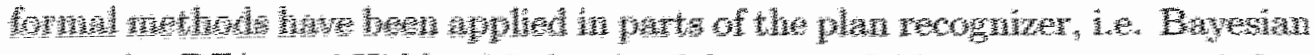

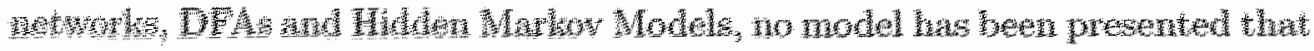

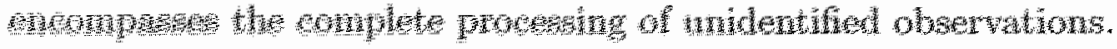

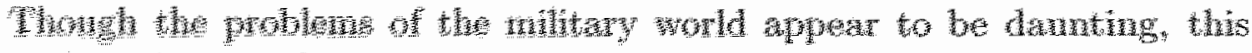

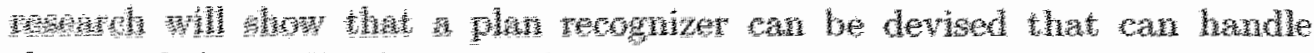

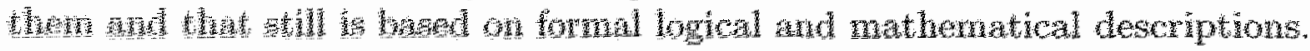

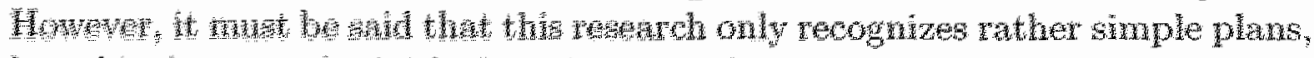

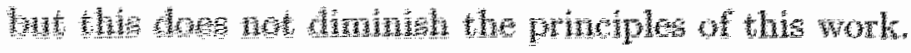

\subsection{Problen statement}

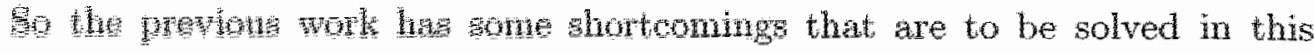

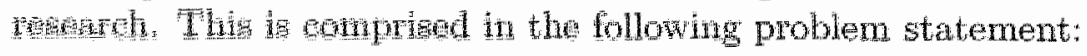


Can a sound, complete, optimal and feasible software system be devised for the Tactical Plan Recogrition problem, that can process unidentified abservations directly?

Our concept sounclness and completeness of an algorithm are described in detail in definitions 5.6 (page 105) resp. 5.7 (page 105). The optimality requirement states that for the sub-tasks of the system, there is a definition of optimality. Our definition of optimality is found in section 5.6 (page 105). The system will try to find the most optimal solutions for the sub-tasks. In this report, we consider an algorithm to be feasible if a simple program, a demonstrator, can be programmed that implements the algorithm. So that a plan recognizer can actually be build with the proposed methods ${ }^{1}$. The last statement about direct processing of unidentified observations refers to an approach in which observations are correlated directly with hypothetical plans rather than to other observations.

\subsection{Addressing the problem statement}

The solution to the problem statement consists of the application of two methods to Tactical Plan Recognition. They will be discussed in the following subsections.

\subsubsection{Research approach}

Research into Tactical Plan Recognition should be viewed as systems research: developing a system composed out of a number of subsystems. Rather than focussing an individual subsystem (e.g. a planning algorithm), the research aims at the development of a comprehensive plan recognition system. The 'tools of the trade', that are used here, are applied logic and applied probability theory.

\footnotetext{
${ }^{1}$ In operations research the term feasible solution means a solution that satisfies constraints, but in this report feasible refers to an avalable demonstrator to can show the workings of the required processing.
} 


\subsubsection{The Multiple Hypothesis Tracking approach for Tactical Plan Recognition}

In this section, the first contribution of this research is presented. Our plan recognition system follows traditional tracking naturally. The input of our system are tracks and intelligence from human observers. The tracking information is augmented with an agent's hypothetical plans and intentions. Longer term prediction of the agent's state and actions is now possible. A successful and optimal tracking algorithm is the Multiple Hypothesis Tracking (MHT) algorithm, that will be introduced below.

The MHT approach to Tactical Plan Recognition entails the adaptation of Reid's MHT, published in 1979 [74], for this plan recognition problem. For this adaptation, it is recognized that:

1. tracking algorithms do a simple form of plan recognition, and

2. it is recognized that some problems, that are associated with Tactical Plan Recognition systems, have already been addressed and solved optimally in a maximum likelihood sense by the MHT.

The work of Reid is mainly based upon that of Sittler (published in 1964!) [81]. Sittler devised a maximum likelihood estimator for the measurementtrack association part of the tracking problem. Reid was the first to devise an algorithm for it. Reid's original MHT algorithm was devised for handling observations from only one sensor, but it is easily adapted to a multi target multi sensor tracking problem in which information of various sensors is fused to form one set of tracks. The case of multiple sources of information to be fused also occurs in Tactical Plan Recognition. From a plan recognition point. of view such a tracking system can be viewed as a (rather simple) very short term movement-plan recognizer. Then it is attractive to examine whether this movement-plan recognizer can be adapted to recognize the medium term (or tactical) enemy plan.

\subsubsection{Application of classical abduction to Tactical Plan Recog- nition}

The second contribution is based on classical abduction as defined by C. S. Pierce. The concepts of classical abduction can readily be applied to plan recognizers for identified observations, as has been done by Lin and Goebel [50] 
and Bauer and Paul [12]. In this work, an adaptation of Lin and Goebel's approach to the case of plan recognition with unidentified observations is given. Plan recognition will be formally defined in the more general context of Tactical Plan Recognition. In addition to plan hypotheses, one also has to generate assigament hypotheses here. The assignment hypotheses link agents' hypothetical plans to observations.

The proposal will be illustrated by working out some simple examples that are still tractable for the reaclers. Although the examples are simple, they clarify how having knowledge about possible plans of objects allows for much longer predictions of the (likely) future behavior of the objects than can be obtained by tracking approaches which only take the kinematic state of the objects into account.

Now the problem statement has been given, we finish the first part with the outline of the thesis, followed by a summary of the first part. In the subsequent parts II until IV, the system for Tactical Plan Recognition is presented in detail.

\subsection{Outline of this thesis}

This document is organized in a manner that is generally found in phased software development. The phases of software development are: requirements, analysis, design, implementation and testing. All the phases produce a description of its results, except for the implementation phase, that results in the program code.

The thesis consists of four main parts. The first part introduces the Tactical Plan Recognition problem, other solutions to this problem and discusses shortcomings of other systems. Then the system 'requirements' are stated in the problem statement. Then it introduces the approach taken in this thesis that will address the problem statement. The remainder of the thesis describes this approach to Tactical Plan Recognition. The software phases are allocated to the parts of this document as follows:

- part I gives an introduction to the Tactical Plan Recognition world, gives a discussion of related work and then gives the problem statement.

- Part II contains the formal description of Tactical Plan Recognition and the task analysis. The task analysis is done for the complete range 
of Tactical Plan Recognition problems, so it results in a Generic Task Model.

- Part III contains the design of the algorithms and of the information model and discusses the following properties of these algorithms: soundness, completeness and complexity.

- Part IV describes the evaluation test-bed, then some experiments that are done with the algorithms and, based on the results, gives an evaluation of the algorithms' performance. Finally conclusions of this research are drawn.

Now the contents of the four parts will be summarized:

- In the first part, Introduction, the problem area, will be introduced. The introduction first describes the military world and Command and Control systems for the military world. Then an overview of plan recognition research in general is given.

Then, planning and plan recognition tasks of the military Command and Control system are mentioned and related to each other in a proposed Generic Task Model of a Command and Control system. Also, four other models and architectures for military Command and Control systems from the literature will be mentioned.

Then we will focus on the problem at hand: Tactical Plan Recognition. We start with some examples that will be used throughout the book. These examples clearly show that a Tactical Plan Recognizer is faced with assignment hypotheses rather than plan hypotheses.

Then other work on the Tactical Plan Recognition problem is discussed. In the following section, some shortcomings of other work are mentioned. Then the problem statement states my goals for this research: to develop a sound, complete, optimal and feasible Tactical Plan Recognizer. Finally, the contributions, of this work, that address the shortcomings of other work are listed.

The first part finishes with a, summary of it.

- Analysis of Tactical Plan Recognition. This part consists itself of two sub parts. In the first part, a formal description of Tactical Plan 
Recognition is given. The relevant objects, involved in a plan recognition process, are defined and related to each other. Then various types of plan recognition and their characteristics are described in terms of classical abduction. This sub part ends with a description of some general properties of a Tactical Plan Recognizer: some properties of the hypotheses, a formal definition of the soundness and completeness and two definitions for its optimality.

The second sub part focusses on the analysis. The Tactical Plan Recognition problem is further analyzed. Descriptions of the processing and information flows of tactical plan recognizers are given by means of Generic Task Models. These models give some insight in the processing and are the basis for the development of the algorithms for plan recognizers.

- The third part is called: Design of Tactical Plan Recognition Algorithms. In this part, algorithms are proposed and analyzed for an identified observation plan recognizer and an unidentified plan recognizer.

First, algorithms for some important sub tasks are examined. Then an algorithm for the simpler identified observation plan recognition problem is presented in chapter 8 . Then its formal properties are examined. The soundness and completeness criteria for the algorithms are stated in the formal descriptions of the previous part. In chapter 9 a plan recognition algorithm for unidentified observations is presented.

- The fourth part of the thesis, Evaluation of Tactical Plan Recognizers the implementation of the Tactical Plan Recognizer (for mnidentified observations) is tested on two scenarios: one army and one naval scenarios. This is presented in chapter 10. Finally chapter 13, (Conclusion) gives the conclusions and further work for this research on Tactical Plan. Recognition. 


\section{Summary of part I}

Part I starts with a general introduction to the world of planning and to the military world with which Command and Control Systems are faced. After the introduction, an overview of the plan recognition field of research is given. The different applications of plan recognition are listed.

After this, the focus is on Command and Control systems, of which the Tactical Plan Recognition task is a part. The role of planning and plan recognition in the Command and Control world, is elucidated by means of a Generic Task Model. In similar models from literature, plan recognition and planning are not explicitly mentioned. So no link to the body of research of plan recognition is recognized. Thus, these models do not guide the model's user to scientific work on parts of the Command and Control system. The Generic Task Model for Command and Control systems, presented here, clearly displays the interactions between the tasks of reactive planning of the own forces and the plan recognition of the enemy's forces.

Then we look at Tactical Plan Recognition in detail, by means of two examples. One example from the world of civil aviation and one example from the army world. The army example clearly shows that, for unidentified observations, a hypothesis tree that represents all possible assignments of observations to hypothetical plans must be erected. So one has to define assignment hypotheses rather than only plan hypotheses, to tackle the Tactical Plan Recognition problem.

After the examples, the approaches of other authors to Tactical Plan Recognition are discussed in the chapter about related work. The related work showed approaches that have a different focus as the work presented here. In the related work there is little attention for the problem of handling unidentified observations. Only a sequential approach for this is presented or the system only takes identified observations as input. Also many of the systems are rather heuristic and do not apply formal logical and mathematical 
models throughout the system. The discussion leads to a problem statement for the research presented in this thesis.

Then the methods for addressing the issues of the problem statement of this research are stated. The methods that are chosen to address the problem statement are given below.

The first method concerns the application of the Multiple Hypothesis Tracking approach to Tactical Plan Recognition. This approach differs significantly from previous work. In the MHT approach, two functions that are nomally separated: correlation and plan recognition, are integrated. Thus, the information of plan hypothesis can be fully exploited in the plan recognition process. Another advantage of this approach is that it calculates the probability of assignment hypotheses and a ranking of assignment hypotheses is possible now and the optimal (that is best) assignment hypothesis can be found.

The second method concerns the application of classical abduction for the formal definition of Tactical Plan Recognition. In the formal definition the input and output information of a Tactical Plan Recognizer is related and described by means of logical formulas of classical Piercian abduction. The formal definitions can be used to examine the soundness of Tactical Plan Recognition algorithms and this research aims at finding sound and complete algorithms for Tactical Plan Recognition. 


\section{Part II}

\section{Analysis of Tactical Plan Recognition}




\section{Chapter 4}

\section{Introduction to Plan recognition}

In the previous part a first description of plan recognition was provided. In this chapter, a detailed and more formal description of the input, the processing and the output of a plan recognizer will be given. First the different plan recognition problems are organized in classes. Then a formal introduction to plan recognition is presented. In the following section two main classes of plan recognition are discerned: the deterministic (logical) one and a probabilistic type of plan recognition that applies statistical models to handle uncertainties.

\subsection{Classifications of Plan Recognition problems}

In this section different classes of plan recognition, from literature and by means of the characteristics of the plan hypotheses, are given. First the characteristics of plans that one finds in the real world are discussed. This mainly focusses on the level of completeness of the plans. Then the various classes of Plan Recognition are presented and discussed. Finally the Plan Recognition classes and plan characteristics of this research are mentioned.

\subsubsection{Characteristics of plans}

A plan is composed out of actions. Planning is done before acting because one expects that the actions may not be generated adequately at plan execttion time. Nevertheless the time-span between planning and action can vary 
between years or weeks down till seconds. For each planning problem there is a solution with a specific granularity. Granularity denotes the 'grain-size' of the plan parts. A plan that counts as a solution is a complete plan. Its granularity is determined by what appears as an entity or an atomic action. An incomplete plan is normally called a partial plan.

Partial plans are coarse-grained and can be represented by the following two basic schemes:

- Skeletal plan : in a skeletal plan, the structure of the plan is considered, but not its contents. A skeletal plan is a template for fitting parts of plans. It has a coarse granularity because it only accounts for (completely specified) crucial aspects (e.g. attributes). If the crucial aspects apply to all potential parts of a (partial) plan, then the skeletal plan defines a context (legal substitutions) for elaborating a skeletal plan part. E.g. in figure 4.1, page 65 the route Amsterdam-Rome should go through Munich.

Two implementation methods for skeletal plans are:

- Partial Order Plan: the sequence of actions of the plan. In the case of incomplete sequence of actions of a plan it is called a Partial Order Plan. If the sequence of actions is completely known it is called a Total Order Plan or linear plan ${ }^{1}$. In fact, in a partial order plan, the plan's actions are related to each other by a partial order relation, $\preceq$, whereas the the actions of a linear plan are related by a total order relation $<$.

- Partially instantiated plan: the number of logical variables that are instantiated. In the case of partial instantiation of a plan it can be called a Partial Instantiated Plan. An example of a partially instantiated plan is the following sequence of actions: $F F y(x$, Frankfurt, Amsterdam), Stop( $x$, Amsterdam)/, where $x$ is a variable that is not instantiated.

- Hierarchical plan: a hierarchical plan consists of parts (called sub plans) which are defined up to some level of composition, but generally must be further refined (by adding more detailed sub plans) to become a

"There has been some terminological confusion about the term linear plan, but Genesereth and Nilsson [31] denote a Total Order Plan as a linear plan. 
complete plan. In this case the coarse granularity takes the form of not allowing details that do not matter. E.g. in figure 4.1 (page 65) the route Amsterdam-Rome is defined by two less abstract parts. A formal representation of this plan is: Go(Amsterdam, Rome $) \Leftrightarrow G o($ Amsterdam, Munich) $\wedge$ Go(Munich,Rome). ${ }^{2}$

Amsterdam

Munich

Rome

Skeletal plan

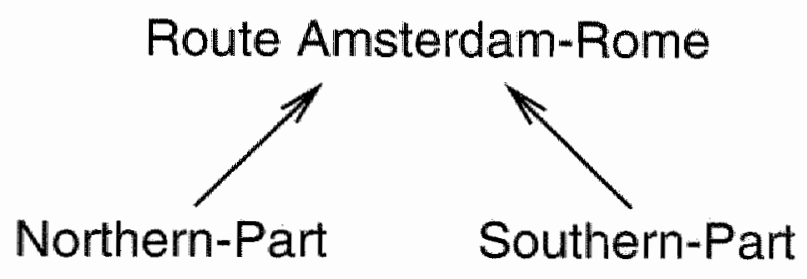

Hierarchical plan

Figure 4.1: Types of planning templates.

Plans can also be discerned as to whether they concern: 1) a single agent executing a plan or 2) multiple agents executing a plan. This distinction is used in the work of Azarewicz.

A military doctrine is always partial: it represents a wide range of complete plans. It also encompasses reasonable variations in the execution of a plan and reasonable variations in the observation of actions resulting in probabilistic, uncertain or unidentified observations.

\subsubsection{Classifications of Plan Recognition}

Two classes of plan recognition problems that are generally distinguished are: intended plan recognition and keyhole plan recognition. The definition of Kautz is as follows $[4]$ :

\section{Intended plan recognition / keyhole plan recognition,} in intended plan recognition, but not keyhole, the recognizer can assume that the observed agent is deliberately structuring its activities in order to make its intentions clear.

\footnotetext{
${ }^{2}$ Note that the hierarchical plan has a composition-decomposition relation (part-of) with its parts and not an generalization-specialization relation (is-a).
} 
So in intended plan recognition the observed agents may even cooperate with the plan recognizer to make their plans clear. This case is always found in cases of teamwork.

An example of intended plan recognition one finds in driving cars. Here the drivers make their intentions clear to other drivers in order to prevent accidents. They communicate their intentions to other drivers by means of signals and signs.

Also in the civil aviation example the flights do not attempt, in any way, to hide their plans. They communicate their flight plans on forehand to air traffic control and are in constant touch with air traffic control during the execution of the flight plan.

In the case of keyhole plan recognition the observed agents do not assist the plan recognizer in understanding their plans. It is quite clear that in our military case we find keyhole plan recognition. Remember the covertness of enemy operations and, of course, the fact that missions and operational plans are kept secret from the enemy.

Recognition problems are also classified as to whether the observer has a complete knowledge of the planning world and whether the agent may perform erroneous plans. Again it is clear that in our case the observer only has incomplete knowledge of the world and that an enemy agent may perform erroneous plans.

\subsubsection{Overview of Plan Recognizers of this research}

In the table 4.1 the classification characteristics, the range of the characteristics and whether the characteristic applies to the Tactical Plan Recognition case are displayed. In the 'TPR' column, the cases that apply Tactical Plan Recognition are indicated by an ' $\mathrm{X}$ '. 'The applicability of these cases are dictated by the military environment.

As we have seen before, the plans have some characteristics. These are summarized in table 4.2. Again the applicable types of recognized plans of this research is indicated by the 'TPR' column. The previous classes of Plan Recognition were dictated by the military environment. The selections made for the types of plans is more a matter of convenience. The most simple types are chosen. Nevertheless, their simplicity will not invalidate the results of this work. Tackling more complex cases can be seen as an extension of the current work. 


\begin{tabular}{|l|c|c|}
\hline characteristic & range & TPR \\
\hline \hline covertness & keyhole plan recognition & $\mathrm{X}$ \\
\hline & intended plan recognition & \\
\hline domain knowledge & complete knowledge & \\
\hline & partial knowledge & $\mathrm{X}$ \\
\hline erroneous plans & possibly & $\mathrm{X}$ \\
\hline & not possible & \\
\hline
\end{tabular}

Table 4.1: Plan recognition classes and characteristics.

\begin{tabular}{|l|c|c|}
\hline characteristic & range & $\begin{array}{c}\text { plans } \\
\text { in this } \\
\text { research }\end{array}$ \\
\hline \hline level of abstraction & non-hierarchical plans & $\mathrm{X}$ \\
\hline & hierarchical plans & \\
\hline sequence of actions & partial order & \\
\hline & total order & $\mathrm{X}$ \\
\hline logical variable instantiation & literals & $\mathrm{X}$ \\
\hline & partial instantiation & \\
\hline stochastic variables & yes & $\mathrm{X}$ \\
\hline & no & \\
\hline number of agents & single-agent & $\mathrm{X}$ \\
\hline & multi-agent & \\
\hline
\end{tabular}

Table 4.2: Plan hypothesis characteristics and the selection of this research. 


\subsection{A formal introduction to Plan Recognition}

In this chapter, first formal definitions for plan recognition are introduced. For this, the traditional traditional plan recognition problem is first defined by means of classical abduction. Then the characteristics of the information used by the plan recognizer: plans, plan templates and plan hypotheses are described in detail.

\subsubsection{Formal definition}

In this section we start with the formal definition of the classical plan recognition problem. It is given below.

Definition 4.1 A plan recognition problem is a pair $(\mathcal{O}, \Gamma)$, where $\mathcal{O}$ is a set logical formulas representing observations and $\Gamma$ is a set of formulas representing the background knowledge. It is assumed that the observations are not entailed by the background knowledge: for all $o \in \mathcal{O}, \Gamma \neq 0^{3}$.

A solution of the plan recognition problem $(\mathcal{O}, \Gamma)$ is a plan hypothesis $\phi$ which satisfies the following two conditions.

1. $\phi$ is consistent ${ }^{4}$ with the observations and the background knowledge. Formally: $\Gamma \cup \mathcal{O} \not \neg \phi^{5}$.

2. $\phi$ explains the observations given the background knowledge. Formally: for all $o \in \mathcal{O}, \Gamma \cup\{\phi\} \vDash o$.

Obtaining a solution to a plan recognition problem is similar to drawing inferences using classical abduction, which can be described as the process of conjecturing explanatory hypotheses. For example, if you are told that all ravens are black $(\forall x(R(x) \Rightarrow B(x)))$, and you observe that a bird $b$ is black $(B(b))$, then you can conjecture that $b$ is a raven $(R(b))$. In this case, the

\footnotetext{
'The 'logical entaiment' relation $F$, like in $A \models B$, means that if the logical formula. $A$ is true, then also $B$ is true. Logical entailment is equivalent to the "provable" relation as in $A \vdash B: A \models B \equiv A \vdash B$.

4 A knowledge base (KB) of logical formulas is inconsistent when it contains both a logical sentence and its negation: $K B=\{\cdots, s, \neg s, \cdots\}$.

"A popular misconception here is that a double negation will result in a positive version of the formula, but this is not the case: $\Gamma \cup \mathcal{D} \neq-\phi \neq \Gamma \cup \models \phi$.
} 
background knowledge $\Gamma$ is the set $\{\forall x(R(x) \Rightarrow B(x))\}, \mathcal{O}$ is the set $\{B(b)\}$, and the formula $R(b)$ is consistent with the observations together with the background knowledge, and it explains the observation given the background knowledge.

A more realistic example is found in diagnosis of a disease by a physician. For each patient, the physician is faced with the diagnosis of the patient's disease. This is done on the basis of symptoms that are mentioned by the patient. The task of the physician to find the disease that causes the symptoms. For this the physician proposes various hypothetical diseases that can cause the symptoms and so can explain the symptoms. In the case that there are multiple hypotheses, the physician has to rank them and decide on the best possible hypothesis. Only when the diagnosis is done and a disease to explain the symptoms is available can the physician propose a cure.

In general, there exist of course many explanatory hypotheses. In the above raven example, the formula $B(b) \wedge F(b)$, representing " $b$ is black and can fly", could in principle also be conjectured. Which candidate explanatory hypotheses are appropriate depends on the application area of abductive reasoning. This is one of the main reasons why in the literature many different formalizations of abduction can be found. See e.g. Aliseda-LLera [3] for a discussion of the many interpretations of abduction.

A popular method for reducing the size of the set of possible explanatory hypotheses is to restrict these possible hypotheses to instantiations of a limited set generic hypothesis descriptions. In the case of plan recognition, the considered explanatory hypotheses can be restricted to plan hypotheses that are instantiations from a limited set of generic plan descriptions (also called plan templates). Therefore, the background knowledge has to contain a set of plan templates in addition to formulas describing knowledge of the planuing world.

It should be remarked that when we allow a plan recognizer to generate partial plans, so we cannot assume that the generated plan hypotheses are mutually exclusive in their parts. Also, in general, we will not assume that the plan hypothesis generation algorithm generates all (in the real world) possible complete plan hypotheses. In the following section we will address these problems by means of Kautz's and Allan's Closed World Assumptions.

In the following sections, partial plans are used as plan templates or, in military terms, model doctrines. 


\subsubsection{Closed World Assumptions}

The background knowledge can be considered to be exhaustive. If a model of the actions is an exhaustive description of the ways actions can be executed, then the two closed world assumptions of Kautz and Allan can be applied. These are recalled below:

Assumption 4.1 Kautz's and Allan's first closed world assumption: the (by the plan recognizer) known ways of performing an action are the only ways to perform the action.

So only the doctrines that are modelled in the background kmowledge of the plan recognizer account for a definition of completeness of the plan recognizer.

Assumption 4.2 Kautz's second closed world assumption: all actions are purposeful and actions can be distinguished from events and that all possible reasons (plans) for performing an action are known.

A consequence of the last assumption is that the plan recognizer is not required to recognize erroneous execution of plans. After all an erroneously executed action serves no purpose. The closed world assumptions impose a bias on the reasoning of the Tactical Plan Recognizer. But the CWAs are very handy in the application of probability to our problem. If the set of plan hypotheses is not complete, it is rather difficult to assign probabilities to hypotheses. Here knowledge of all events is necessary.

\subsubsection{Plan hypotheses}

\subsubsection{Partial or complete plan hypotheses}

After the discussion about plan templates, one has to decide about using partial or complete plans in the plan hypotheses. If a plan hypothesis is partial one may have to interleave planning and plan recognition. The sequence of actions of a partial plan hypothesis are be incomplete and its variables may be partially instantiated. As new observations enter the plan recognition system, the operators are unified with the observations and thus substitutions for the variables become available. When a new observation is entered into the system and unification with a plan hypothesis is not possible or it does not fit a partial order of actions, it is not correlated to a plan hypothesis. 
Whether a partial plan hypothesis suffices also depends on the user's requirements on the plan recognizer. Sometimes the user will require an output of complete plans, but it is also possible that a higher level of abstraction is preferred by the user.

There are some advantages to supply completely instantiated plans as plan hypotheses:

- Correlation with new observations is only useful if the plan hypotheses are sufficiently refined.

- If the plan is complete, constraints on the plan can be examined. For example: if one states the geographic start point of an object's trajectory and, given the current position of the object, one can check whether the endurance of the object suffices to be able to execute the plan hypothesis.

- by supplying complete plans all the planning is done before correlation and it is not necessary to inter leave planning and correlation of observations with plans. The interleaving of planning and correlation is rather complex.

Disadvantages of complete plan hypotheses are:

- the complete set of plan hypotheses may become large in complex cases.

- sometimes it is not really possible to generate complete plans, because all objects that are involved in the plan are not yet observed.

\subsubsection{The plan hypothesis' life cycle}

When the first observations enter the plan recognition system, a new set of plan hypotheses is created for each of them. These plan hypothesis are of type New Plan (NP). An important question, in designing a plan recognizer, is how to incorporate new information of the observed world in the system's plan hypotheses. This new information is obtained from successive observations. One can either choose to adapt a current set of plan hypotheses to new observations, but also choose to start up new plan hypotheses for these new observations.

In the case of identified and certain (deterministic) observations, many of these new plan hypotheses will already exist in the initial set of plan hypotheses. This property results from Azarewicz's first closed world assumption, assumption 4.1 , that states that all doctrines are known by the plan recognizer. 
So the initial set of plan hypotheses explains, not only the first observation, but a subset of the initial plan hypotheses is guaranteed also to explain and be consistent with any successive observations. If, at some time, the complete initial set of plan hypotheses becomes inconsistent with some new observation, the knowledge of enemy doctrines is incomplete. This violates Azarewicz's first closed world assumption.

In the case of some uncertainty in the parameter values of identified observations, some parts of a plan hypothesis may be adapted to new observations. By example, updating of the estimated kinematic variables of the plan. Such an updated plan hypothesis is always based upon a previous plan hypothesis and a new observation. These updated plan hypotheses are of type Correlated Plan (CP). This adaptation does not affect the 'logical' explanation and consistency properties. The plan hypotheses still are closely linked to the only target of the observations.

When a plan hypothesis has not been updated for a while and when the plan's targets have been detected to execute the final parts of the plan, one may assume that the plan's targets have left the coverage of the sensors or the plan has been executed completely. In this case the plan hypothesis can be removed from the system and is a Lost Plan (LP).

In the case of unidentified observations, the situation concerning plan hypotheses seems to be different. Here, for each new measurement, a new set of plan hypotheses have to be created, because each new observation may originate from a new object. The new object is not yet entailed in the set of plan hypotheses.

Now the general description of the plan hypotheses is finished. In the next chapter we will focus on the uncertainties in both observations and plan hypotheses.

\subsection{Uncertainties of observations and plan execu- tion}

In the previous chapter, certain and deterministic observations were assumed. In this chapter, uncertainties of the observations and of the plan execution are discussed. First four different types of uncertainty in the observations are presented. Then the uncertainties of plan execution itself are given. 


\subsection{UNCERTAINTIES OF OBSERVATIONS AND PLAN EXECUTION73}

\subsubsection{Plans, states and actions}

Planning is changing a planning world from an initial state to a required state. The required state is described by way of a number of goals. The planner executes actions that transform the planning world from the initial state to the goal state. An observation concerns the actual state of the world or actual changes of the world state by actions. A state is represented by a conjunction of grounded function free literals. An observation can refer to an observed action, e.g. $G o(K L 174$, Heathrow), or an observed state, e.g. At(KL174, Heathrow). States as well as actions will be observed, therefore define:

Definition 4.2 An action observation refers to an observed step in some plan to be recognized. A state observation refers to some observed state of agents and objects during the execution of some plan to be recognized.

A plan consists of actions, so subsequent state observations will be translated into action observations. The action is that action that that will cause the state changes. Examples of action hypotheses are: Buy(Frank, Drill) and Fly(Frank, Amsterdam,Heathrow). Examples of state observations are: At(Frank, Amsterdam), Has(Frank, Drill).

Given the previous states: $\neg$ Has $($ Frank, Drill) and At(Frank, Frankfurt) one can easily determine the action that caused the state change: Buy(Frank, Drill) respectively Fly(Frank, Frankfurt, Amsterdam).

State observations are certainly useful for assessing the probability of hypotheses, if the uncertainty in the state of an object can be modelled by stochastic variables.

A plan is completely observable when all its actions can be observed by a sensor or human observer. Mostly one is interested in more significant actions that are often observable. By example, one is only interested in the observation that an airplane is flying from London to Amsterdam, but not in the individual steering actions of the pilot or auto-pilot during this flight.

In control theory two types of uncertainty are taken into account when observing a physical process: measurement noise and process noise. When applied to plan recognition these concepts are defined as:

- measurement noise: uncertainties in the observations, introduced by the limitations of the observer. This is the subject of section 4.3 .2 . 
- Process norse: uncertainties in the execution of a plan. This is the topic of section 4.3 .3

\subsubsection{The observations}

Let's elaborate on the measurement noise. One can distinguish four types of observations, one certain observation, called an identified observation, and three types with some form of uncertainty introduced by the observer. The different types of observations are elaborated below:

- Identified observations: here the object or objects involved in an action are identified. Each object is uniquely identified and the observer can tell what objects from different observations are identical. An example is: the airplane KL174 is flying to Heathrow. Here the observer has read the name of the airplane and knows that it executes the scheduled flight KL174 to Heathrow. All the relevant facts of the observation are known in the identified observation. It is formally a literal (function free, grounded). Formally the observation tells us: Fly (KL174, Heathrow)

- Unidentified observations: here the observer cannot read the name of the airplane, he only sees one aircraft flying to one destination. If the observer is near Heathrow, he might infer that the destination of the aircraft is Heathrow. Of course a classified type of an object: Heli, Prop,Jet,... can be positioned between an identified and an unidentified object. In first order logic this is modelled by an extra predicate like Heli( $x)$. The observations of the example of a flight to Heathrow then have the form: there is an airplane flying to Heathrow (1). When also the destination is unknown: there is an airplane flying to a destination (2). So in the case of an unidentified observation some objects appearing in the observation are unknown. So formally the observations of the examples are:

(1) $\exists x\{F l y(x$, Heathrow $)\}$

(2) $\exists x, y\{F l y(x, y)\}$

Another interesting formalization uses, not the existential quantifier, but the 'there is only one' quantifier: $\exists$ !. We then get a proposition like $\exists ! x\{F l y(x$, Heathrow $)\}$ However, there are no simple reasoning systems that can handle this quantifier. As the existential quantifier suffices to illustrate the reasoning about unidentified observations, it will be used in this document. 


\subsection{UNCERTAINTIES OF OBSERVATIONS AND PLAN EXECUTION75}

- Probabilistic observations: now the observer can estimate some variables of the observations. E.g. the observer can estimate the speed of flight KL174 to be approximately $200[\mathrm{~km} / \mathrm{h}]$. So the observation looks like: there is one aircraft flying to Heathrow with an approximate speed of $200[\mathrm{~km} / \mathrm{h}]$. Formally we can model the uncertainty of the speed probabilistically, e.g. with a Gaussian probability density function: $v \sim \mathcal{N}(200[\mathrm{~km} / \mathrm{h}], 30[\mathrm{~km} / \mathrm{h}])$. Formally the observation then is:

$$
F l y(K L 174, \text { Heathrow, } v), v \sim \mathcal{N}(200[\mathrm{~km} / \mathrm{h}], 30[\mathrm{~km} / \mathrm{h}]))
$$

- Uncertain observations: in this case one does not trust the observer to make a correct observation. Only a percentage of these observations are actually true. A degree of belief, in our case a probability, can be defined for such an observation: e.g. Confirmed (95\%), Probable (70\%) and NotProbable (25\%). An uncertain observation is sometimes represented by adding a prime to the symbol of the observation: if the certain observation is o then its uncertain observation is denoted as $o^{\prime}$. Formally the following propositions apply for an example of an uncertain observation:

$$
\begin{gathered}
P(\text { Fly }(K L 174, \text { Heathrow }) \mid O b s(F l y(K L 174, \text { Heathrow })))= \\
\text { Confirmed }=95 \% \\
P\left(o \mid o^{\prime}\right)=\text { Confirmed }
\end{gathered}
$$

These types of observations, that a Tactical Plan Recognition system has to be able to handle, are summarized in table 4.3 .

The type of observation also determines the type of generated bypotheses and the amount of detail that a hypothesis can have. The observations and their hypotheses are listed below.

- Identified abservations: the hypotheses can only be logical propositions about plan hypotheses.

- Unidentified abservations: one must generate logical hypotheses about plans and logical assignment hypotheses. 


\begin{tabular}{|c|c|}
\hline Observation type & example \\
\hline Identified observations & the airplane KL174 is going to Heathrow \\
\hline Unidentified observations & $\begin{array}{l}\text { - there is one airplane going to Heathrow } \\
\text { - there is one airplane going to one destination }\end{array}$ \\
\hline Probabilistic observations & $\begin{array}{l}\text { the airplane } \mathrm{KL} 174 \text { is going to Heathrow } \\
\text { with an approximate speed of } 200[\mathrm{~km} / \mathrm{u}]\end{array}$ \\
\hline Uncertain abservations & $\begin{array}{l}\text { There is a Confirmed observation of } \\
\text { airplane KL } 174 \text { going to Heathrow }\end{array}$ \\
\hline Observation type & Formal representation of example \\
\hline Identified observations & $\overline{\text { Fly(KL174, Heathrow })}$ \\
\hline Unidentified observations & $\begin{array}{l}\exists x\{F l y(x, \text { Heathrow })\} \\
\exists x, y\{F l y(x, y)\}\end{array}$ \\
\hline Probabilistic observations & $\begin{array}{l}\text { Fly }(\text { KL } 174, \text { Heathrow, v), } \\
v \sim \mathcal{N}(200[\mathrm{~km} / \mathrm{h}], 30[\mathrm{~km} / \mathrm{h}])\end{array}$ \\
\hline Uncertain observations & $\begin{array}{l}O_{1}=O b s(F l y(K L 174, \text { Heathrow })) \\
P\left(\text { Fly }(K L 174, \text { Heathrow }) \mid O_{1}\right)= \\
\text { Confirmed }=95 \%\end{array}$ \\
\hline
\end{tabular}

Table 4.3: Different types of observations 


\subsection{UNCERTAINTIES OF OBSERVATIONS AND PLAN EXECUTION77}

- Observations with measurement time: now the plan hypotheses also have the current time of execution.

- Observations with measurement time and measured position: now the plan hypotheses have current time and the plan recognizer can estimate the kinematic state of the targets. Having the estimated kinematic state and the measurement time, one can estimate the begin time of the plan execution and the begin time of the execution of particular legs of the plan hypotheses. But now, no two plan hypotheses for one object are equal, as they contain estimated times and kinematic state.

- Uncertain observations: one way to handle the account for this is to adapt the detection probability and false alarm rate of the observer's model. But one might also consider applying a bayesian network here. In this report, the correct processing of this uncertainty is not examined.

The type of observations that are entered in the plan recognition system, of course, have great impact on its processing. In this thesis the uncertain observations are not handled yet. This because no theory for handling uncertain observations is available yet. No theory for this case of observations is developed in this thesis because it has no bearing on the principles of Tactical Plan Recognition that are presented here.

\subsubsection{The plan execution}

With regard to the execution of a tactical plan one is uncertain about:

1. erroneous execution: this problem is not considered in this thesis, it conflicts with Azarewicz's assumption that all actions performed are purposeful (assumption 4.2),

2. localization uncertainty: this can be modelled by a probability density function along the planned trajectory.

3. partial achievement of goals: abort actions are explicitly modelled in the plan template.

So this chapter mentioned various uncertainties in observations and plan execution. It shows how one can model and handle these most of these uncertainties. 
Now we have studied in the plan recognition process, we can define formal properties of the plan recognition process, given the character of the uncertainties. This will be the topic of the next chapter. 


\section{Chapter 5}

\section{Deterministic and probabilistic Plan Recognition}

In this chapter, the definition of plan recognition will be refined. Two classes of plan recognizers will be distinguished. The first are deterministic plan recognizers. These have identified or unidentified observations as input. As the background knowledge is also assumed to be certain, such a recognizer will output a set of plan hypotheses. Note that these hypotheses are not ranked and it is not possible to select the $k$-best hypotheses. The second class are probabilistic plan recognizers. These have as input: identified, unidentified and probabilistic observations. Their output is a number of assignment hypotheses, ranked by the probability of an assignment hypothesis.

\subsection{Deterministic Plan Recognition}

The simplest type of deterministic plan recognition concerns the recognition of only one plan, executed by one agent. This coincides with the earlier given definition of "traditional" plan recognition. The formal definition of traditional plan recognition is repeated below:

Definition 5.1 A (single) plan recognition problem is a pair $(0, \Gamma)$, where $\mathcal{O}$ is a set of formulas representing observations and $\Gamma$ is a set of formulas representing the background knowledge. It is assumed that the observations are not entailed by the background knowledge: for all $o \in \mathcal{O}, \Gamma \not \models o$. A solution of the plan recognition problem $(\mathcal{O}, \Gamma)$ is a plan hypothesis $\phi$ 
which is consistent with the observations and the background knowledge, and explains the observations given the background knowledge.

Of course, typically, one is not satisfied with finding a single solution to a plan recognition problem, but prefers to generate several solutions, from which the most interesting hypotheses can be selected.

The observations can be either identified or unidentified, but in the above definition it is assumed that they all concern the same plan. Also, any plan hypothesis of is required to explain all observations. This is adequate in the case of a single-agent plan. However, when there are multiple agents executing multiple independent plans, a plan hypothesis should only be required to explain the observations originating from the same plan.

For the case of multiple plans, we need to propose several hypothetical plans at once and add information about the subset of the observations that is supposed to be explained by each hypothetical plan. For this purpose, we define the following notion of an assignment of observations to hypothetical plans.

Definition 5.2 Given a set of observations $\mathcal{O}$ and a set of generated plan hypotheses, $\Phi$, an assignment $\Psi_{h}$ is a surjective function $\Psi_{h}: \mathcal{O} \longrightarrow \Phi$, with as domain the set of observations and as codomain ${ }^{1}$ the set of generated hypotheticall plans.

An assignment incorporates hypotheses about which observations originate from the same object. The codomain of $\Psi_{h}$ only consists of the plan hypotheses generated for the observations, and not of all possible plan hypotheses. Therefore, we can assume $\Psi_{h}$ to be surjective. Moreover, we can use the notation $\Psi_{h}(\mathcal{O})$ for the set of plan hypotheses generated for the observations $\mathcal{O}$, i.e. the codomain.

Given an assignment $\Psi_{h}$, the set of observations $\mathcal{O}$ is partitioned into equivalence classes of observations that originate from the same plan. We write $\|o\|_{h}$ for the equivalence class $\left\{o^{\prime} \in \mathcal{O} \mid \Psi_{h}\left(o^{\prime}\right)=\Psi_{k}(o)\right\}$ of observation $o$.

Recognition of multiple independent plans can now essentially be defined as several single plan recognitions, where each single plan recognition is limited

\footnotetext{
${ }^{1}$ The term codomain and image are often confused. In Stanati [82] a function is defined as: $f: A \rightarrow B$, then $A$ is the domain, $B$ is the codomain. Regarding the image: it is related to a subset of the domain. Define $f\left(A^{\prime}\right)=f(a) \mid a \in A^{\prime}, A^{\prime} \subset A$. Then $f\left(A^{\prime}\right)$ is the image of $A^{\prime}$ under $f$. The image of the entire domain, $f(A)$, is called the image of the function $f$. and apparently is identical to the codomain.
} 
to an equivalence class of observations.

Definition 5.3 A multiple independent plan recognition problem is a pair $(\mathcal{O}, \Gamma)$, where $\mathcal{O}$ is a set formulas representing observations (not necessarily concerning a single plan) and $\Gamma$ is a set of formulas representing the background knowledge. It is assumed that the observations are not entailed by the background knowledge: for all $o \in \mathcal{O}, \Gamma \neq 0$, and that the background knowledge does not contain constraints between possible plans. A solution of the plan recognition problem $(\mathcal{O}, \Gamma)$ is a set of plan hypotheses $\Phi$ and an assignment $\Psi_{h}: \mathcal{O} \longrightarrow \Phi$ such that, for each $o \in \mathcal{O}$, the following two conditions are satisfied.

1. $\Psi_{h}(0)$ is consistent with the observations assigned to $\Psi_{h}(0)$ and the background knowledge. Formally: $\Gamma U\|o\|_{h} \not \neq \rightarrow \Psi_{h}(o)$.

2. $\Psi_{h}(o)$ explains the observations assigned to $\Psi_{h}(o)$ given the background knowledge. Formally: for all $o \in\|o\|_{h}, \Gamma \cup\left\{\Psi_{h}(o)\right\} \models o$.

If the plans are dependent, then the plans have to be consistent with the background knowledge and all the observations rather than the equivalence class of an observation. So, as all the plans are related, so are the observations. Each plan hypothesis only has to explain the observations assigned to it. The complete definition is found in Mulder and Voorbraak [66]. In the following text, we only will consider the independent case that suffices for this introductory text.

In many sensor data fusion systems, the measurements or observations are organized in distinctive sets called scans. The measurements of such a scan, observe the target at about the same time. The scans form a partition of the complete set of observations. Moreover one can rely on the fact that in a scan, there is only one observation per target per sensor or observer. So define a scan:

Definition 5.4 The scans of a set of abservations is a partition of this set. During a scan each object in the coverage of the sensors and observers is observed only ance by each sensor or observer.

For a rotating sensor, such as a search radar, a scan corresponds with one rotation of the sensor. So define the assignment of the current scan as follows: 
Definition 5.5 An assignment of the current scan $k$, is a surjective function $\Psi_{k, k_{k}}: \mathcal{O}_{k} \longrightarrow \Phi_{k-1, h} \cup\left\{N P_{h}\right\}$, with as domain the set of observations of the current scan $\mathcal{O}_{k}$ and as codomain the set of hypothetical plans of the previous scan augmented with the set $N P_{i}$, that indicates the new plan set of scan $i$. The set of hypothetical plans of the previous scan is given by:

$$
\Phi_{k-1, h}=\bigcup_{i=0}^{k-1}\left(N P_{i}-D P_{i}\right)
$$

Here and $D P_{i}$ represents the terminated plan set of scan $i$. A plan hypotheses is terminated, when no observation for it has been received during a timeout period. After the time-out, the plan hypotheses is deemed not to exist anymore and is removed from the plan recognition system. $\square$

The total assignment hypothesis consists of that of the current scan, $\Psi_{h}$, concatenated with those of the previous scans, $\Omega_{g}^{k}:\left(\Psi_{h}: \Omega_{g}^{k-1}\right)$. The set of hypothetical plans is split up in plans that already existed in the previous scans minus the terminated plans and new plans that are created in the current scan by observations of the current scan. Each scanning sensor only gives one observation for each observed object per scan. In this case it is a bijective assignment function for the current scan for each sensor.

\subsection{Examples}

In this section the formal definitions will be applied to the examples of chapter 2.3. The examples start rather simple, but are gradually complicated until in the last example we get a realistic Tactical Plan Recognition problem. An overview of the characteristics of the examples is given in table 5.1 .

\subsubsection{Plan hypotheses}

Now consider the example 2.1 from chapter 2.3 about flight plans in civil aviation.

Example 5.1 Flight KL174 and KL175: In a first example consider two aeroplanes S ("Swean") and Z ("Zwaluw") which can be used for two routes: KL174 from $H$ (Heathrow) to F (Frankfurt) and KL175 from Frankfurt to Heathrow. Both flights stop in A (Amsterdam). This results in four possible 


\begin{tabular}{|l||c|c|c|c|}
\hline example & id & time & agents & hypothesis \\
\hline \hline 5.2 .1 & id & no & single & plan \\
\hline 5.2 .2 & id & no & single & plan \\
\hline 5.2 .4 & id & yes & single & plan \\
\hline 5.2 .6 & id & no & single & plan \\
\hline 5.2 .7 & id & no & multi & assignment \\
\hline 5.2 .8 & id & no & multi & assignment \\
\hline 5.2 .9 & unid & no & multi & assignment \\
\hline 5.2 .10 & unid & no & multi & assignment \\
\hline 5.2 .11 & unid & no & multi & assigmment \\
\hline 5.2 .12 & unid & no & multi & assignment \\
\hline
\end{tabular}

Table 5.1: Characteristics of the examples.

plans: Flight(KL174,S), Flight(KL174,Z), Flight(KL175,S), Flight(KL175,Z). The background knowledge $\Gamma$ contains the following formulas:

$$
\begin{aligned}
& \forall x F l i g h t(K L 174, x) \Leftrightarrow F l y(H, A, x) \wedge \operatorname{Stop}(A, x) \wedge F l y(A, F, x) \text { and } \\
& \forall x F l i g h t(K L 175, x) \Leftrightarrow F l y(F, A, x) \wedge \operatorname{Stop}(A, x) \wedge F l y(A, H, x) .
\end{aligned}
$$

These formulas are 'plan templates' from which actual plan hypotheses can be derived. Note that the background knowledge does not contain any information about the aeroplanes $\mathrm{S}$ or $\mathrm{Z}$. For simplicity we abstract from temporal considerations, and represent flights as conjunctions of their steps. So there is no ordening that applies to the actions.

Suppose we have the observation: $o=\operatorname{Stop}(A, Z)$, then two viable hypotheses are: Flight $(K L 174, Z)$ and Flight $(K L 175, Z)$.

Observations. Note that the observation cannot be derived from the background knowledge, because the background knowledge does not contain specific information about the aeroplanes Swean or $Z$ waluw, so: $\Gamma \not 0$. Also note that the background knowledge, when combined with the observation does not contradict the plan hypothesis: $\Gamma \cup\{o\} \neq \neg \phi$.

Consistency. Substitution of $Z$ for the variable $x$ results in a set of plan hypotheses 
$\{$ Flight $(K L 174, Z)$, Flight $(K L 175, Z)\}$. As these are only hypothetical, there is no logical sentence about mutual exclusion of these hypotheses in the background knowledge. In fact there is no negative proposition in all in the background knowledge. So not any proposition about inconsistency can be inferred.

Explanation. Finally, given the plan hypotheses we can explain the observations: $\Gamma \cup\{\phi\} \models o$. Substitution of $\mathrm{Z}$ for $\mathrm{x}$ infers $\operatorname{Stop}(A, Z)$ from both sentences of the background knowledge.

Next consider a somewhat more difficult example. The situation is graphically displayed in figure 5.1. The icons of the units are accompanied by their unit number, for example, the enemy air force base in the upper left corner is unit $\mathrm{U} 1 \mathrm{in}$ the examples.

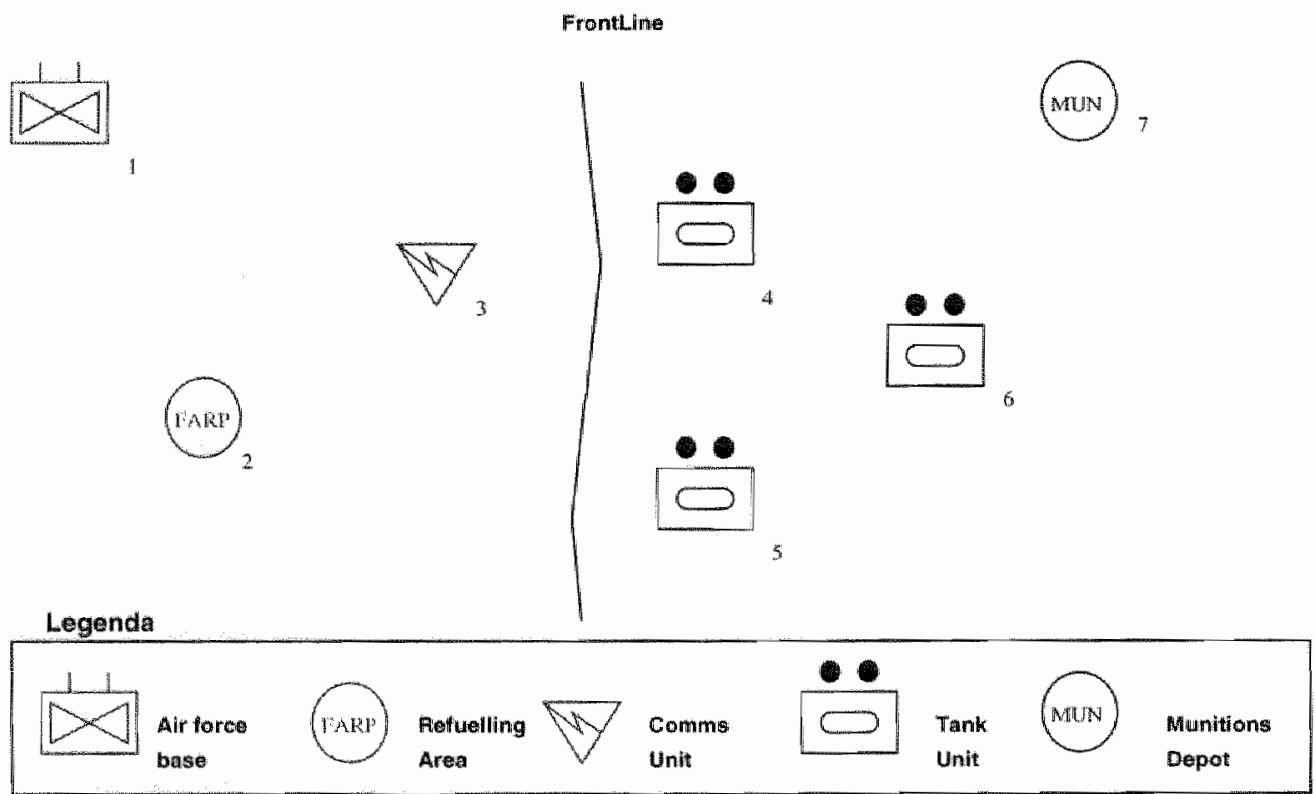

Figure 5.1: The example tactical situation.

Example 5.2 Army Tactical Plan Recognition example: Consider an attack helicopter $\mathrm{H} 1$ and a start base U1, a target U5 and a return base FARP. The background knowledge now is: $\{F o e(U 1)$, Foe $(F A R P)$, Friend(U4), 
Friend(U5), Friend(U6)\}, combined with rules about possible plans: $\forall x, a, b, c$ $\operatorname{Attack}(x, a, b, c) \Leftrightarrow F l y(x, a, b) \wedge \operatorname{Attack}(x, b) \wedge F l y(x, b, c)$.

Suppose we have the observation: $o=F l y(H 1, U 1, U 5)$, then a viable hypothesis is: Attack $(H 1, U 1, U 5, F A R P)$.

Observations. Note that the observation cannot be derived from the background knowledge, again because the background knowledge does not contain any information of a particular observed agent (in this example $H 1$ ), so: $\Gamma \not 0$.

Consistency. Substitution of $\mathrm{H} 1$ as $\mathrm{x}, \mathrm{U} 1$ as a, U5 as b and FARP as c, does not result in a contradiction with the observation. From the substitution we can only derive Attack $(H 1, U 1, U 5, x)$ that does not contradict the input hypothesis Attack ( $H 1, U 1, U 5, F A R P)$. We certainly cannot derive a proposition contradicting the attack of unit $U 5$. The actions involved in the substitution become:

$F l y(H 1, U 1, U 5) \wedge A \operatorname{tack}(H 1, U 5) \wedge F l y(H 1, U 5, x) . F l y(H 1, U 5, x)$ does not contradict $F l y(H 1, U 1, U 5)$, so the consistency check goes: $\Gamma \cup\{o\} \notin \neg \phi$.

Explanation. Finally examine the explanation property: $\Gamma \cup\{\phi\} \models o_{*}$ Again the substitutions $\{x / H 1, a / U 1, b / U 5, c / F A R P\}$ combine the plan hypothesis with the background knowledge and the Fly $(H 1, U 1, U 5)$ observation can be inferred. So also the explanation property holds.

\subsubsection{Temporal considerations}

In the examples above, no temporal considerations were modelled. As a consequence the order of the steps of the plan does not matter. This is a far cry from the actual situation, so it is desirable to model order of steps of the plans in some manner. One can impose this order of the steps of a plan hypothesis on the sequence of observations. If the sequence does not fit the order, the plan hypothesis is considered to be inconsistent with the observationss.

To model an ordering in the plan steps and thus also the observations, we add a total order relation, also called linear order, to the background knowledge. For the first example (example 5.1) this relation is: 
Example 5.3 Imposing a total order relation, $<$, on the steps of a plan:

\begin{tabular}{ll}
$\begin{array}{ll}\text { plan } \\
\text { template }\end{array}$ & $\forall x F l i g h t(K L 174, x) \Leftrightarrow F l y(H, A, x) \wedge \operatorname{Stop}(A, x) \wedge F l y(A, F, x)$ \\
\hline$<\quad$ & $\{F l y(H, A, x)<\operatorname{Stop}(A, x), F l y(H, A, x)<F l y(A, F, x)$, \\
& $\operatorname{Stop}(A, x)<F l y(A, F, x)\}$ \\
\hline
\end{tabular}

The transitive elements of the total order relation are not mentioned in example 5.3. The logical representation of the total order, $<$, is:

\begin{tabular}{ll}
\hline$<:$ & $\{F \operatorname{ly}(H, A, x)<\operatorname{Stop}(A, x), F \operatorname{Fly}(H, A, x)<F l y(A, F, x)$, \\
& $\operatorname{Stop}(A, S)<F l y(A, F, S)\} \equiv$ \\
\hline logical: & $(F \operatorname{ly}(H, A, x)<\operatorname{Stop}(A, x)) \wedge$ \\
& $(F l y(H, A, x)<F l y(A, F, x)) \wedge$ \\
& $(\operatorname{Stop}(A, x)<F l y(A, F, x))$, \\
\hline anti symmetric: & $\forall x, y, x \neq y,(x<y) \Rightarrow \neg(y<x)$ \\
\hline
\end{tabular}

Of course, the total order set translates logically to a conjunction of its elements. Next consider the logical implication that specifies the anti symmetric property. In a partial order relation, $\preceq$, the anti symmetric property is normally stated as: $\forall x, y,(x \leq y) \wedge(y \leq x) \Rightarrow x=y$. This cannot be applied for the total order relation, $<$, as this is irreflexive: one cannot make a statement $x<x$ in a total order relation. Therefore the anti symmetric property is stated as above with a negation.

How is the total order relation integrated in the logical framework? The observations are considered in isolation, and the observer itself has no knowledge of the relation of its observations to other observations. The plan recognition system itself must register the sequence in which it receives the observations and imput this in the reasoning system.

Example 5.4 Suppose we have the situation of example 5.1. Now suppose we have received the following sequence of observations: $[\operatorname{Stop}(A, S)$, $F l y(H, A, S)]$.

When this is combined with the plan hypothesis of Flight $(K L 174, S)$, that flies from Heathrow to Franlsfurt via Amsterdam we get the following 
sentences:

\begin{tabular}{|c|c|}
\hline$\Gamma:$ & $\begin{array}{l}\{F \operatorname{light}(K L 174, x) \Leftrightarrow F \operatorname{Fly}(H, A, x) \wedge \operatorname{Stop}(A, x) \wedge F \operatorname{ly}(A, F, x)\} \cup \\
\{F l y(H, A, x)<\operatorname{Stop}(A, x), \\
F \lg (H, A, x)<F l y(A, F, x), \\
\operatorname{Stop}(A, S)<F \lg (A, F, S)\}\end{array}$ \\
\hline$\phi:$ & Flight $(\bar{K} L 174, S)$ \\
\hline & $\{$ Stop $(A, S), F l y(H, A, S)$, Stop $(A, S)<F l y(H, A, S)\}$ \\
\hline
\end{tabular}

Observations. From the background knowledge $\Gamma$ there is no way in which we can derive the sequence $[S \operatorname{Stop}(A, S), F l y(H, A, S)]$ or its representation as a logical conjunction: $\operatorname{Stop}(A, S) \wedge F l y(H, A, S) \wedge S t o p(A, S) ; F l y(H, A, S)$. Simply because the symbol ' $S$ ' (Swaen) is not present in the background knowledge.

Consistency. When the background knowledge is combined with the observations, substitute $S$ for $x$ and infer: $F$ light $(K L 174, S) \Leftrightarrow F l y(H, A, S) \wedge$ $\operatorname{Stop}(A, S) \wedge F l y(A, F, S) \wedge(F l y(H, A, S)<\operatorname{Stop}(A, x)) \wedge(F l y(H, A, S)<$ $F l y(A, F, S)) \wedge(S t o p(A, S)<F l y(A, F, S))$. The antisymmetric property, applied on the $F l y(H, A, S)<S \operatorname{top}(A, S)$ element, states that $\neg(S \operatorname{top}(A, S)<$ $F l y(H, A, S))$. However, the observation contained the literal: $(\operatorname{Stop}(A, S)<$ $F l y(H, A, S)$ ), so we have in the knowledge base (i.e. current set of sentences) a sentence and its negation: $\{s, \neg S\}$, hence an inconsistency (here $s$ is $(\operatorname{Stop}(A, S)<F l y(H, A, S)))$.

Explanation. When the plan hypothesis is combined with the background knowledge, it unifies with the left hand side of the equivalence about Flight $(K L 1 / 4, x)$. From this we can derive the complete instantiated plan for 'Swean'. So also the observed prefix of the complete sequence of plans can be derived.

The example above showed that adding an order relation in the background knowledge, allows checks on the order in which the observations are received. If the observation's sequence is inconsistent with the partial order that is defined for a plan template, the formal properties do not apply to the sequence and the plan hypothesis is rejected. 


\subsubsection{Assignment hypotheses}

Now again apply the civil aviation example 2.1 of chapter 2.3 to our formal definitions. The example concerns aircrafts and their flight plans.

Example 5.5 We consider two airplanes S("Swaen") and Z("Zwaluw") which can be used for two routes: KL174 from H (Heathrow) to F (Frankfurt), and KL175 from Frankfurt to Heathrow. Both flights stop in A (Amsterdam). This results in four plans: Flight(KL174, S), Flight(KL174, Z), Flight(KL175, S) and Flight $(K L 175, Z)$. The background knowledge $\Gamma$ contains the following formulas: $\forall x$ (Flight $(K L 174, x) \Leftrightarrow F l y(H, A, x) \wedge \operatorname{Stop}(A, x) \wedge F l y(A, F$, $x))$ and $\forall x(F \operatorname{Fight}(K L 175, x) \Leftrightarrow F l y(F, A, x) \wedge \operatorname{Stop}(A, x) \wedge F l y(A, H, x)) \square$

For simplicity, we first abstract from temporal considerations, and represent the flights as conjunctions of parts. Now we start with one observation concerning the 'Swean' flight.

Example 5.6 Suppose we have the observation $o_{1}=\operatorname{Stop}(A, S)$. This observation $o_{1}$ can be assigned to either Flight $(K L 174, S)$ or Flight(KL175, S). Both plan hypotheses are consistent with $\Gamma \cup\left\{o_{1}\right\}$, and explain $o_{1}$ given the background knowledge. In this case, the assignments are not really necessary, since it concerns a single plan. The formal requirements are discussed below in detail.

Observations. The background knowledge cannot infer the observations in any way, as it does not contain information about the objects Swean or Zwaluw, i.e. $\Gamma \not \equiv a$.

Consistency. The background knowledge when combined with the observation, is consistent with the plan hypothesis. The logical equivalences of the background knowledge, combined with the observation, can only infer positive propositions about Flight $(K L 174, S)$ and $F$ light $(K L 175, S)$. There is no way to derive either $\neg$ Flight $(K L 174, S)$ or $\neg$ Flight $(K L 175, S)$, as the background knowledge does not have any negations. Neither some closed world assumption goes. So $\Gamma \cup \circ \not \models \neg \phi$.

Explanation. Finally both plan hypotheses Flight $(K L 174, S)$, Flight $(K L 175, S)$ can derive $S t o p(A, S)$ by generalized modus ponens when 
combined with the logical equivalence of the plan template in the background knowledge, hence they both can explain the observation.

Example 5.7 Suppose the observations are $o_{1}=S \operatorname{top}(A, S)$, and $o_{2}=S \operatorname{Stop}(A, Z)$. Now both observations $o_{1}$ and $o_{2}$ can be assigned to either flight KL174 or flight KL175. This results in four plans:

1. Flight(KL174, S), Flight(KL175, Z).

2. Flight(KL175, S), Flight(KL174, Z).

3. Flight(KL174, S), Flight(KL174, Z).

4. Flight(KL175, S), Flight(KL175, Z).

The last two plans can be excluded by adding some constraints between the possible plans. For example, $\forall x \forall y$ (Flight $(x, y) \Rightarrow \forall z($ Flight $(x, z) \Rightarrow y=z))$ represents the constraint that each flight is performed by at most one airplane.

The formal requirements are discussed in detail now. The background knowledge, again, cannot derive any propositions about objects, such as Swean or Zwaluw. They are not part of the knowledge base of the background knowledge.

Assignment hypothesis. The assignment hypothesis is in this case:

$$
\begin{aligned}
\Psi_{h}= & \left\{o_{1} \rightarrow \text { Flight }(K L 174, S), o_{1} \rightarrow \text { Flight }(K L 175, S)\right. \\
& \left.o_{2} \rightarrow \text { Flight }(K L 174, Z), o_{2} \rightarrow \text { Flight }(K L 175, Z)\right\}
\end{aligned}
$$

The equivalence classes are $\left\|o_{1}\right\|_{h}=\left\{o_{1}\right\},\left\|o_{2}\right\|_{h}=\left\{o_{2}\right\}$.

Consistency. Now the consistency requirements are in this example:

rU $\left\|o_{1}\right\|_{h} \not \neg \neg$ Flight $(K L 174, S)$, Flight $\left.(K L 175, S)\right\}$ for the first equivalence class and

$\Gamma \cup\left\|o_{2}\right\|_{h} \not \neg\{F l i g h t(K L 174, Z)$, Flight $(K L 175, Z)\}$ for the second equivalence class. 
Explanation. The hypotheses are also required to be able to explain the observations of an equivalence class. In our example this becomes:

$\forall 0 \in\left\|o_{1}\right\|_{h}, \Gamma \cup\{F l i g h t(K L 174, S)$, Flight $(K L 175, S)\} \vDash o$, that is:

$\Gamma \cup\{$ Flight $(K L 174, S)$, Flight $(K L 175, S)\} \models o_{1}$.

Similarly for the second equivalence class:

$\forall o \in\left\|o_{2}\right\|_{h}, \Gamma \cup\{$ Flight $(K L 174, Z)$, Flight $(K L 175, Z)\} \vDash o$, and that is:

$\Gamma \cup\{$ Flight $(K L 174, Z)$, Flight $(K L 175, Z)\} \models o_{2}$.

In the following example, the two airplanes are observed.

Example 5.8 Suppose the observations are $o_{1}=S \operatorname{top}(A, S), o_{2}=S \operatorname{top}(A, Z)$, and $o_{3}=F l y(A, F, S)$. Now the only possible assignment for $o_{1}$ and $o_{3}$ is Flight $(K L 174, S)$. The remaining observation $o_{2}$ can be assigned to either Flight $(K L 174, Z)$ or Flight $(K L 175, Z)$ if no constraints between the flights are present. With the constraints mentioned above only Flight $(K L 175, Z)$ remains possible.

Assignment hypothesis. The assignment hypothesis is in this case:

$$
\begin{aligned}
\Psi_{h}= & \left\{o_{1} \rightarrow \text { Flight }(K L 174, S), o_{1} \rightarrow \text { Flight }(K L 175, S),\right. \\
& \left.o_{2} \rightarrow \text { Flight }(K L 174, Z), o_{3} \rightarrow \text { Flight }(K L 174, Z)\right\}
\end{aligned}
$$

The equivalence classes are $\left\|o_{2} \mid=\left\{o_{2}\right\},\right\| o_{1}\left\|_{h}=\right\| o_{3} \|_{h}=\left\{o_{1}, o_{3}\right\}$.

Consistency. Now the consistency requirements are in this example: $\Gamma \cup\left\|_{1}\right\|_{h} \not \models \neg\{F \operatorname{light}(K L 174, S)\}$ for the first equivalence class and $\Gamma \cup\left\|o_{2}\right\|_{h} \not \neq \neg\{F l i g h t(K L 174, Z)$, Flight $(K L 175, Z)\}$ for the second equivalence class.

Explanation. The hypotheses are also required to explain the observations of an equivalence class. In our example this becomes:

$\forall o \in\left\|o_{1}\right\|_{h}, \Gamma \cup\{$ Flight $(K L 174, S)\} \models o$, that is:

$\Gamma \cup\{F$ light $(K L 174, S)\} \models o_{1}$ and $\Gamma \cup\{F$ light $(K L 174, S)\} \models o_{3}$ Similarly for the second equivalence class:

$\forall o \in\left\|o_{2}\right\|_{\hbar}, \Gamma \cup,\{F l i g h t(K L 174, Z)$, Flight $(K L 175, Z)\} \models o$, that is:

$\Gamma \cup\{$ Flight $(K L 175, Z)$, Flight $(K L 175, Z)\} \vDash=o_{2}$. 
In the following example an unidentified observations is input to the plan recognizer.

Example 5.9 Now suppose we have an unidentified observation $o_{1}=\exists x \operatorname{Stop}(A, x)$. This observation $o_{1}$ can be assigned to all four plans Flight $(K L 174, S)$, Flight $(K L 175, S)$, Flight $(K L 174, Z)$ and Flight $(K L 175, Z)$.

Observations. Consider the requirement, that the observation cannot be deduced from the background knowledge. On a first glance, one would expect to be able to deduce the observation from the logical implications. However, the logical implications simple state that, if and only if an object is executing a fight plan, then it will execute the flight plan's steps. It does not state that actually there is an object that is executing the Hight plan, i.e. $\exists x \operatorname{Stop}(A, x)$.

The existential quantifier also allows multiple objects to execute the same flight plan: $\exists x \operatorname{Stop}(A, x)$ says that one or more objects are executing the flight plan. The 'there is only one' quantifier is also interesting to be used: $\exists ! x \operatorname{Stop}(A, x)$, but (as mentioned before) this will not be pursued in this research.

Consider the following two propositions about red objects: $\forall x A p p l e(x) \Leftrightarrow$ $\operatorname{Red}(x)$, stating that all apples are red and $\exists y R e d(y)$, stating that there is an object that is red. If we can derive $\exists y \operatorname{Red}(y)$ from $\forall x A p p l e(x) \Leftrightarrow \operatorname{Red}(x)$ we have a problem. However, this derivation is not possible because the 'for all' quantifier only links apples with the red color, but does not state that there always are apples in the knowledge base. Only when one is sure that there are apples in the knowledge base, one can state that there always are one or more objects that are Red.

Consistency. The next question is whether the observation is consistent with the hypothetical plans. From the existential observation, combined with the logical implications of the background knowledge, one can only derive positive propositions about plans. It is impossible to derive the negation.

Explanation. The final requirement is that the plan hypotheses can explain the observation. The plans relate to $\operatorname{Stop}(A, S)$ and $S \operatorname{top}(A, Z)$. It states that there are one or more objects that stop in Amsterdam, i.e. $\exists x S t o p(A, x)$.

In the following example, the next observation concerns the observation of a fly action. 
Example 5.10 Suppose we have two unidentified observation $o_{1}=\exists x \operatorname{Stop}(A, x)$ and $o_{2}=\exists x \mathrm{Fly}(A, F, x)$. Let us assume that the background knowledge contains the constraint that each flight is performed by at most one airplane. Then the following four multiple plans remain possible:

1. Flight (KL174, S), Flight (KL175, Z). Here observation $o_{1}$ is assigned to Flight $(\mathrm{KL174}, \mathrm{S})$ and $o_{2}$ to Flight $(\mathrm{KL} 175$, Z).

2. Flight(KL175, S), Flight(KL174, Z).

Here observation $o_{1}$ is assigned to Flight(KL175, S) and $o_{2}$ to Flight(KL174, Z).

3. Flight(KL174, S).

Here observations $o_{1}$ and $o_{2}$ are both assigned to Flight(KL174, S).

4. Flight(KL174, Z).

Here observations $o_{1}$ and $o_{2}$ are both assigned to Flight (KL174, Z).

Next consider the, more difficult, army example 2.3 of section 2.3 . The situation is graphically displayed in figure 2.6.

Consider an attack helicopter $\mathrm{H} 1$ and a start base $\mathrm{U} 1$, a target U5 and a return base FARP. The background knowledge now is:

$\{$ Foe $(U 1)$, Foe $(F A R P)$, Friend(U4), Friend $(U 5)$, Friend $(U 6)\}$, combined with rules about possible plans:

$\forall x, a, b, c$ Attack $(x, a, b, c) \Leftrightarrow F l y(x, a, b) \wedge \operatorname{Attach}(x, b) \wedge F l y(x, b, c) \wedge F o e(x) \wedge$ Foe $(a) \wedge$ Friend $(b) \wedge$ Foe $(c)$.

Example 5.11. Suppose we have the observation: $o=F l y(H 1, U 1, U 5)$, then a viable hypothesis is: $\operatorname{Attack}(H 1, U 1, U 5, F A R P)$.

Observations. Note that the observation cannot be derived from the background knowledge, again because the background knowledge does not contain any information about the agent, denoted by the constant $H 1$, of the observation, so: $\Gamma \neq 0$. Substitution of $H 1$ as $x, U 1$ as $a, U 5$ as $b$ and FARP as $c$, does not result in a contradiction with the observation. From the substitution we can only derive Attack $(H 1, U 1, U 5, x)$ that does not contradict the input hypothesis Attack ( $H 1, U 1, U 5, F A R P)$. 
Consistency. The actions involved in the substitution become: $F l y(H 1, U 1, U 5) \wedge \operatorname{Attack}(H 1, U 5) \wedge F l y(H 1, U 5, x)$. As no temporal considerations are modelled in the background knowledge, $F(y(H 1, U 5, x)$ does not contradict $F l y(H 1, U 1, U 5)$, so the consistency check goes: $\Gamma \cup\{0\} \not \neg \phi$.

Explanation. Finally examine the explanation property: $\Gamma \cup\{\phi\} \vDash 0$. Again the substitutions $\{x / H 1, a / U 1, b / U 5, c / F A R P\}$ combine the plan hypothesis with the background knowledge and so the $F l y(H 1, U 1, U 5)$ observation can be inferred. So also the explanation property goes.

Example 5.12 Suppose we have the observation: $o=\exists x F l y(x, U \mathbb{1}, U 5)$, then a viable hypothesis is: Attack $(x, U 1, U 5, F A R P)$.

Observations. The observation cannot be derived from the background knowledge. The logical implication $\forall x, a, b, c$ Attack $(x, a, b, c) \Leftrightarrow F l y(x, a, b)$ $\wedge \operatorname{Attack}(x, b) \wedge F l y(x, b, c)$ closely links Fly steps with the Attack plan. However, it does not state that there actually is a Fly step being executed by someone, so: $\Gamma \not \neq o$. Substitution of, $U 1$ as $a, U 5$ as $b$ and $F A R P$ as $c$, does not result in a contradiction with the observation. From the substitution we can only derive Attack $(x, U 1, U 5, y)$ that does not contradict the input hypothesis $\operatorname{Attack}(x, U 1, U 5, F A R P)$.

Consistency. The actions involved in the substitution become:

$F l y(x, U 1, U 5) \wedge A t t a c k(x, U 5) \wedge F l y(x, U 5, y)$. As no temporal considerations are modelled in the background knowledge, $F l y(x, U 5, y)$ does not contradict $F l y(x, U 1, U 5)$, so the consistency check goes: $\Gamma \cup\{0\} \not \forall \rightarrow \phi$.

Explanation. Finally examine the explanation property: $\Gamma \cup\{\phi\} \models o$. Again the substitutions $\{a / U 1, b / U 5\}$ combine the plan hypothesis with the background knowledge, and so the $F l y(x, U 1, U 5)$ observation can be inferred. So also the explanation property holds.

Now we have finished the examination of various forms of discrete plan recognition. It has been shown that the abduction properties apply. Next we will look into probabilistic plan recognition. This can be applied if probabilistic observations with stochastic variables or uncertain observations are the input of the plan recognizer. An advantage of probabilistic plan recognition is that 
the plan hypotheses or assignment hypotheses kan be ranked and that the $k$-best of them can be selected.

\subsection{Probabilistic Plan Recognition}

The previous sections concerned deterministic plan recognition with identified or unidentified observations. These are explained by a consistent set of plan hypotheses or assignment hypotheses. These plan hypotheses cannot be ranked directly, as they all explain the observations. In the case of probabilistic or uncertain observations the plan recognizer can calculate the probability: of each plan hypothesis. This can appropriately be called probabilistic plan recognition.

In the case of identified observations, a plan recognizer gives pairs: $(\phi, P(\phi))$, where: $\phi$ is the plan hypothesis and $P(\phi)$ is the probability of the plan hypothesis. However, the set of hypotheses $\Phi$ must be exhaustive and its elements sum of the individual probabilities adds up to one: $\sum P(\phi)=1$.

In the case of unidentified (uncertain or probabilistic) observations the hypothesis becomes the pair: $\left(\Psi_{h}, P\left(\Psi_{h}\right)\right)$ where: $\Psi_{h}$ is the assignment hypothesis and $P\left(\Psi_{h}\right)$ its probability. Here the assignment hypotheses must be exhaustive and mutually exclusive so: $\sum P\left(\Psi_{h}\right)=1$. Note that in the latter case only the probability of the assignment is calculated and not the direct probability of plan hypotheses in the assignment.

In the literature attempts have been made to define some special semantics for abductive inference with evidence augmented with a numerical uncertainty. One has to redefine terms such as consistency and explanation, in a probabilistic setting. For this we refer to the work of Neapolitan in [68] and Nilsson's probabilistic logic in [31].

There is no consensus about probabilistic abduction. But no general method for reasoning about uncertainty has been applied in this research. This because a specific and suitable technique to handle probabilistic, unidentified observations to generate a number of assignment hypotheses and their probabilities, is available from the field of tracking. The adaptation of Reid's method to our problem of Tactical Plan Recognition is described in the following section. 


\subsubsection{Reid's formula}

Tracking algorithms organize unidentified observations of airplanes, ships and vehicles from one or more sensors, called plots, into tracks: sets of plots which are thought to originate from the same object. The, currently, most successful model for tracking is the multiple hypothesis tracking (MHT) algorithm from Reid in [74]. In this algorithm plot - track assignment hypothesis are formulated for each new scan of data, based upon assignment hypotheses from the previous scans. The assignment hypotheses are ranked by Reid's formula (formula 5.3). This formula gives the probability of a set of assignments consisting of $\Omega_{g}^{k-1}$, the assignments of the previous scans and $\Psi_{h}$, the assignment of the current scan $k$, given the set of plots of all the scans, $Z: P\left(\Omega_{g}^{k-1} \mid Z\right)$. An extract of the calculation of the assignment hypothesis of Reid's paper is given below.

By applying Bayes' formula and the chain rule for conditional probabilities we get:

$$
P\left(\Omega_{g}^{k-1}, \Psi_{h} \mid Z\right)=\frac{1}{C} P\left(Z(k) \mid \Omega_{g}^{k-1}, \Psi_{h}\right) P\left(\Psi_{h} \mid \Omega_{g}^{k-1}\right) P\left(\Omega_{g}^{k-1}\right)
$$

The first part of the formula above concerns the probability of a new data set $Z(k)$ of scan $k$, given the assignments of the previous scans. The probability depends on the composition of the set of measurements. Let $M_{k}$ be the number of measurements of $Z(k)$, then:

$$
P\left(Z(k) \mid \Omega_{g}^{k-1}, \Psi_{h}\right)=\prod_{m=1}^{M_{k}} f(m)
$$

where:

- $f(m)=1 / V_{\text {sensor }}$ if the $m^{\text {th }}$ measurement is a false alarm or concerns a new target if it enters the coverage of the sensor. $V_{\text {sensor }}$ is the volume or area covered by the sensor.

- $f(m)=g_{x y}$ when the measurement is from a track whose existence is implied by the prior hypothesis, $\Omega_{g}^{k-1} . g_{x y}$ is the correlation probability between a measurement $x$ and a track $y$ and is approximated by the normal distribution of the residue between the measurement and the track. 
The second part of the right hand side of formula 5.2 concerns the probability of the current assignment hypothesis, given the prior assignment hypothesis, $\Omega_{g}^{k-1}$. The following three steps describe the calctlation:

1. the derivation first considers the probability of a the number of elements of a partitioning of the set of measurements, $M_{K}$, of the current scan in $N_{N T}$ new tracks, $N_{F A}$ false alarms and $N_{D T}$ measurements associated to existing tracks. This probability is calculated by assuming: a binomial distribution for the number of re-detected targets out of $N_{T G T}$ tracks

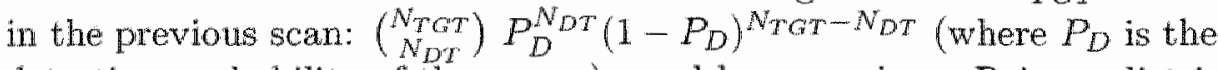
detection probability of the sensor), and by assuming a Poisson distribution for the number of new targets and false alarms: $F_{N_{N T}}\left(\beta_{N T} V\right)$ resp. $F_{N_{F A}}\left(\beta_{F A} V\right)$ (where $\beta_{F A}$ is the false alarm rate of the sensor and $\beta_{N T}$ is the average number of new targets that enters the coverage in each new scan.

2. Secondly consider the probability of a particular configuration of partitioning $M_{K}$ measurements in three components: new tracks, false alarms and correlated measurements.

3. Finally, given the numbers and configuration, determine the probability of a particular assignment of $N_{D T}$ measurements to $N_{T G T}$ existing tracks).

The resulting probability is found by the product of the three probabilities clescribed above and is given by:

$P\left(\Psi_{h} \mid \Omega_{g}^{k-1}\right)=\frac{N_{F A} ! N_{N T} !}{M_{K} !} P_{D}^{N_{D T}}\left(1-P_{D}\right)^{N_{T C T}-N_{D T}} F_{N_{F A}}\left(\beta_{F A} V\right) F_{N_{N T}}\left(\beta_{N T} V\right)$

Substituting the two left parts of the right hand side and simplifying the combined formula, results in the following assignment probability for the single-sensor case:

$$
\begin{aligned}
& P\left(\Omega_{g}^{k-1}, \Psi_{h} \mid Z\right)=\frac{1}{C} P_{D}^{N_{D T}}\left(1-P_{D}\right)^{N_{T G T}-N_{D T}} \\
& \beta_{F A}^{N A} \beta_{N T}^{N_{N T}}\left(\prod g_{x y}\right) P\left(\Omega_{g}^{k-1}\right)
\end{aligned}
$$

where: 
- $C=$ is normalization constant,

- $P_{D}$ is the detection probability of the sensor when the target is in the range of the sensor,

- $N_{T G T}=$ the number of prior targets (tracks) that were recognized by the tracking system in the previous scans,

- $N_{D T}=$ the number of plots of the current scan associated with prior targets of previous scans,

- $N_{F A}=$ the number of plots of the current scan associated with false targets (e.g. birds and sea or land clutter),

- $N_{N T}=$ the number of plots of the current scan associated with new targets in the coverage,

- $\beta_{F A}=N_{F A} / V_{\text {sensor }}$ is the false alarm density per scan of the sensor,

- $V_{\text {sensor }}$ is the volume of a $3 \mathrm{D}$ sensor or the area of a $2 \mathrm{D}$ sensor

- $\beta_{N T}=N_{N T} / V_{\text {sensor }}$ is the new target density per scan of the sensor,

- $\Psi_{h}=$ the assignment of plots of the current scan to prior targets, false targets and new targets,

- $Z=\bigcup_{i=0}^{i=k} Z_{k}$ where $Z_{k}$ the set of $M_{k}=N_{D T}+N_{F A}+N_{N T}$ plots of the current scan,

- $g_{x y}=$ the correlation likelihood between a plot $\mathrm{x}$ and a track $\mathrm{y}, x \in$ $\left[1 . . N_{D T}\right], y \in\left[1 . . N_{T G T}\right]$ This is based on the statistical distance between the observed state of the plot and the predicted estimated state of the track. In the case of gaussian stochastic variables this is the Mahanalobis distance and thus a probability density function. This distance is also applied by a similar application of Mevassvik [52].

The multiple sensor case of tracking with non-overlapping sensors is a simple generalization of the formula above. The observation events of different observers are stochastically indepenclent, so a product of probabilities suffices.

$$
P\left(\Omega_{g}^{k-1}, \Psi_{h} \mid Z\right)=\frac{1}{C} \prod_{s e=1}^{N_{s e n s o r}}\left\{P_{s e, D}^{N_{s e, D T}}\left(1-P_{s e, D}\right)^{N_{T G T}-N_{s e, D T}}\right.
$$




$$
\left.\beta_{s e, F A}^{N_{s e, F A}} \beta_{s e, N T}^{N_{s e, N T}}\left(\prod g_{s e, x y}\right)\right\} P\left(\Omega_{g}^{k-1}\right)
$$

where se $\in\left[1 . . N_{\text {sensor }}\right]$ is a sensor's index.

\subsubsection{Reid's formula for Tactical Plan Recognition}

In the case of plan recognition with probabilistic unidentified observations, parts of Reid's formula are redefined. Recall formula 5.3 for a single observer:

$$
P\left(\Omega_{g}^{k-1}, \Psi_{h} \mid \mathcal{O}\right)=\frac{1}{C} P_{D}^{N_{D T}}\left(1-P_{D}\right)^{N_{T G T}-N_{D T}} \beta_{F A}^{N_{F A}} \beta_{N T}^{N_{N T}}\left(\prod g_{x y}\right) P\left(\Omega_{g}^{k-1}\right)
$$

The main difference with Reid's original formula, is that the correlation likelihood, $g_{x y}$, now is the likelihood of a observation being associated to a prior plan hypothesis of the previous scan, $k-1$,

As mentioned before, we had two types of observations with respect to actions and states: state observations and action observations. Both are used to correlate with plan hypotheses.

1. action observation: The correlation of an action observation with a plan hypothesis consists in part of a check whether the observed actions 'fits' in the sequences of actions generated by the plan hypothesis. If it fits the sequence, the sequence's correlation probability is one, otherwise it is zero. More elaborate schema's utilizing Markov schemes can be devised, but this will not change the workings of the method significantly.

2. state observation: The state observations are used to calculate the correlation likelihood $g_{x y y}$. It gives the statistical distance between the predicted state at the observation time of a plan hypothesis in the assignment and the observed state.

In the following sub-section we utilize the adapted formula of Reid for the running army example of figures 2.6 till 2.12 , page 35 . Here the values of the possible assignment hypotheses are calculated.

\subsubsection{A numerical example of Reid's formula}

If the first observation at time T1, $O_{1}$, enters the system, it is assumed that the base of the observation's helicopter is air base (unit 1). Only two targets are 
considered by the plan recognizer for simplicity: the munitions depot, MUN, and tank unit 5, TK5. And so a plan hypothesis of an attack of a helicopter, $\mathrm{H} 1$, on the munitions depot (MUN): $A(H 1, M U N)$ and a plan hypothesis of an attack of a helicopter, $\mathrm{H} 1$, on unit 5 (TK5): $A(H 1, T K 5)$ are generated. The plans are instantiated from the following plan template: A(heli,target), that consists of the steps:

[Fly(heli, base, comm), CommTarget (heli, comm),

Fly(heli, conm, target), Fly(heli, target, base)]

The three observations tell that a helicopter has been spotted at a certain position. So they concern a position: a state. The observations can match with one of the first two Fly steps: Fly (heli, base, comm), Fly (heli, comm, target).

So after the first observation enters the system, there are two assignment hypotheses, one for each plan hypothesis. Their probabilities are determined by the $\beta_{N T}$.

- $\Psi_{1,1}=\left\{O_{1} \leftrightarrow A(H 1, M U N)\right\}, P\left(\Psi_{1,1}\right)=\frac{1}{C} \beta_{N T}$

- $\Psi_{1,2}=\left\{O_{1} \leftrightarrow A(H 1, T K 5)\right\}, P\left(\Psi_{1,2}\right)=\frac{1}{C} \beta_{N T}$

Next the observation $\mathrm{O}_{2}$ at $\mathrm{T} 2$ generates a new plan hypothesis of helicopter $\mathrm{H} 2$ coming from the FARP and attacking TK5, but it also correlates with the plan of $\mathrm{H} 1$ attacking the TK5 unit. Now there are the following assignment hypotheses:

- $\Psi_{2,1}=\left\{\mathrm{O}_{2} \leftrightarrow A(H 2, T K 5)\right\}$ and the helicopter $\mathrm{H} 1$ is still assumed to attack MUN. The probability of the hypothesis is $P\left(\Psi_{2,1}\right)=\frac{1}{C} \beta_{N T}(1-$ $\left.P_{D}\right) P\left(\Psi_{1,1}\right)$ The plan hypotheses in this assignment are: $A(H 1, M U N)$, A (H2,TK5).

- $\Psi_{2,2}=\left\{\mathrm{O}_{2} \leftrightarrow A(H 2, T K 5)\right\}$ where $\mathrm{H} 1$ is also assumed to attack TK5. So now there are two helicopters, both attacking TK5. The probability here is $P\left(\Psi_{2,2}\right)=\frac{1}{C} \beta_{N T}\left(1-P_{D}\right) P\left(\Psi_{1,2}\right)$ The plan hypotheses in this assignment are again: $A(H 1, T K 5), A(H 2, T K 5)$.

- $\mathbb{I}_{2,3}=\left\{\mathrm{O}_{2} \leftrightarrow A(H 1, T K 5)\right\}$. So now both observations are assigned to the same helicopter $\mathrm{H} 1$ and the probability of the hypothesis is $P\left(\Psi_{2,2}\right)=$ $\frac{1}{C} P_{D g_{x y 21}} P\left(\Psi_{1,2}\right)$. The plan hypothesis in this assignment is: $A(H 1, T K 5)$. 


\begin{tabular}{|c|c|c|c|}
\hline assignment & plan bypotheses & probability & value \\
\hline \multicolumn{4}{|c|}{ ב } \\
\hline$\Psi_{1,1}$ & $\mathrm{~A}(\mathrm{Hl}, \mathrm{MUN})$ & $\frac{1}{c} \beta_{N T}$ & 0.5 \\
\hline$\Psi_{1,2}$ & $\mathrm{~A}(\mathrm{H} 1, \mathrm{TK} 5)$ & $\frac{1}{c} \beta_{N T}$ & 0.5 \\
\hline \multicolumn{4}{|c|}{$\mathrm{O}_{2}$} \\
\hline$\Psi_{2,1}$ & $\mathrm{~A}(\mathrm{H} 1, \mathrm{MUN}), \mathrm{A}(\mathrm{H} 2, \mathrm{TK} 5)$ & $\frac{1}{C} \beta_{N T}\left(1-P_{D}\right) P\left(\Psi_{1,1}\right)$ & 0.24339 \\
\hline$\Psi_{2,2}$ & $\overline{\mathrm{A}}(\mathrm{H} 1, \mathrm{TK} 5), \mathrm{A}(\mathrm{H} 2, \mathrm{TK} 5)$ & $\frac{1}{C} \beta_{N T}\left(1-P_{D}\right) P\left(\Psi_{1,2}\right)$ & 0.24339 \\
\hline$\Psi_{2,3}$ & $\mathrm{~A}(\mathrm{H} 1, \mathrm{TK} 5)$ & $\frac{1}{C} P_{D g_{x y 21}} P\left(\Psi_{1,2}\right)$ & 0.51322 \\
\hline \multicolumn{4}{|c|}{$O_{3}$} \\
\hline$\Psi_{3,1}$ & $\begin{array}{l}\mathrm{A}(\mathrm{H} 1, \mathrm{MUN}), \mathrm{A}(\mathrm{H} 2, \mathrm{TK} 5) \\
\mathrm{A}(\mathrm{H} 3, \mathrm{TK} 5)\end{array}$ & $\frac{1}{C} \beta_{N T}\left(1-P_{D}\right)^{2} P\left(\Psi_{2,1}\right)$ & 0.007380 \\
\hline$\Psi_{3,2}$ & $\mathrm{~A}(\mathrm{H} 1, \mathrm{MUN}), \mathrm{A}(\mathrm{H} 2, \mathrm{TK} 5)$ & $\frac{1}{C} P_{D} g_{x y 31}\left(1-P_{D}\right) P\left(\Psi_{2,1}\right)$ & 0.015562 \\
\hline$\Psi_{3,3}$ & $\begin{array}{l}\mathrm{A}(\mathrm{H} 1, \mathrm{TK} 5), \mathrm{A}(\mathrm{H} 2, \mathrm{TK} 5), \\
\mathrm{A}(\mathrm{H} 3, \mathrm{TK} 5)\end{array}$ & $\frac{1}{C} \beta_{N T}\left(1-P_{D}\right)^{2} P\left(\Psi_{2,2}\right)$ & 0.007380 \\
\hline$\Psi_{3,4}$ & $\mathrm{~A}(\mathrm{H} 1, \mathrm{TK} 5) \mathrm{A}(\mathrm{H} 2, \mathrm{TK} 5)$ & $\frac{1}{C} P_{D} g_{x y 32}\left(1-P_{D}\right) P\left(\Psi_{2,2}\right)$ & 0.015562 \\
\hline$\Psi_{3,5}$ & $\mathrm{~A}(\mathrm{H} 1, \mathrm{TK} 5) \mathrm{A}(\mathrm{H} 2, \mathrm{TK} 5)$ & $\frac{1}{C} P_{D} g_{x y 33}\left(1-P_{D}\right) P\left(\Psi_{2,2}\right)$ & 0.015562 \\
\hline$\Psi, 6$ & $\mathrm{~A}(\mathrm{H} 1, \mathrm{TK} 5) \mathrm{A}(\mathrm{H} 3, \mathrm{TK} 5)$ & $\frac{1}{C} \beta_{N T}\left(1-P_{D}\right) P\left(\Psi_{2,3}\right)$ & 0.155616 \\
\hline$\Psi_{3,7}$ & $\mathrm{~A}(\mathrm{H} 1, \mathrm{TK} 5)$ & $\frac{1}{C} P_{D} g_{x y 34} P\left(\Psi_{2,3}\right)$ & 0.782939 \\
\hline
\end{tabular}

Table 5.2: Assignment hypothesis probabilities in the example. 
The last observation $\mathrm{O}_{3}$ at $\mathrm{T} 3$ gives the best match with the attack of helicopter $\mathrm{Hl}$ of TK5 and the $\Psi_{3,7}$ assignment hypothesis gets the most support:

- $\Psi_{3,7}=\left\{O_{3} \leftrightarrow A(H 1, T K 5)\right\}$, the probability of the hypothesis is $P\left(\Psi_{3,1}\right)=$ $P_{D} g_{x y} P\left(\Psi_{2,3}\right)$. Note that now the relative difference of the probability of this hypothesis and that of the others is larger than in the previons scan.

In table 5.2 all the assignment hypotheses are given. Now assume the following values for the variables of table 5.2:

$$
\begin{aligned}
\beta_{N T}= & N_{N T} / \text { Area }=1 /\left(4\left[\mathrm{~km}^{2}\right]\right)=2.510^{-7} \\
P_{D}= & 0.9 \\
g_{x y}= & \mathcal{N}(v, B) \text { is normally distributed in }(v, B), \text { where } \\
v= & \text { the residue between planned position } \\
& \text { and observed position } v=[d x, d y] \text { and } \\
B= & \text { is the two dimensional cartesian covariance matrix of the residue } \\
(v, B)= & \left([1000,1000],\left[\left[100^{2}, 10^{2}\right]\left[10^{2}, 100^{2}\right]\right]\right) \text { for the following } \\
& \text { correlation probabilities: } g_{x y 21}=g_{x y 31}= \\
& g_{x y 32}=g_{x y 33}=5.8610^{-8} \\
& \left.\left([500,100],\left[100^{2}, 10^{2}\right],\left[10^{2}, 100^{2}\right]\right]\right) \text { for the following } \\
& \text { correlation probability: } g_{x y 34}=1.4010^{-7}
\end{aligned}
$$

After receiving the first observation, $O_{1}$, two assignment hypotheses are created, that cannot be discerned from each other. The second observation, $\mathrm{O}_{2}$, correlates very well with the attack of unit 5 (TK5), and at this stage this hypothesis wins. The third observation, $O_{3}$, also correlates very well with the original attack of wit 5 and so after receiving the final observation still the optimal assignment contains only one plan hypothesis of the attack of unit 5 .

In the previous chapters, plan hypotheses and assignment hypotheses were introduced for describing Tactical Plan Recognition. In the following chapter, some properties of these hypotheses will be stated and proved.

\subsection{Propositions about plan hypotheses and assign- ment hypotheses}

Now we can make some propositions about plan hypotheses that are initiated and updated by an observation. Remember, from section 4.2 .3 , that a plan 
hypothesis is either instantiated from a first plot (NP) or build from a previous plan hypothesis (CP) and a newly correlated plot. So one can define a binary parenthood relation between plan hypotheses. In fact the relation is a quasi order relation, were the parent plan hypothesis is ordered before the child plan hypothesis:

Proposition 5.1 The plan hypotheses in a unidentified observation plan recognizer are related by a quasi order parenthood relation. So define a quasi order set $\langle\Phi, \prec\rangle$ where $\Phi$ is the set of plan hypotheses. The quasi order relation is induced by parenthood of a plan hypothesis of a child plan hypothesis. NP plan hypotheses are not comparable to each other, CP plan hypotheses that descend from the same NP plan hypothesis and the originating NP plam hypothesis are comparable.

Proof. It is not a partial order relation because it is irreflexive: a plan hypothesis is not its own parent. It is not a linear order, because not all plan hypotheses are comparable.

The parenthood relation is of interest in calculating admissible assignments of observations to plan hypotheses.

If we define comparability as an equivalence relation, one can partition the set of plan hypotheses, $\Phi$ in a set of blocks (also called cells or components, i.e. subsets) and each block has one unique least NP element. The partitioning and equivalence relation are also associated to equivalence classes.

The number of blocks of the partition, i.e the rank of the partition, equals the number NP plan hypotheses in the system. It does not equal the number of actual targets that are in the coverage of the observers, times the number of plan hypotheses that is initiated for each target. If there is no pruning of old plan hypotheses, the number of blocks will grow due to new NP plan hypotheses, but also the blocks themselves will grow by new correlations to new measurements. Each such a new correlation is represented by a new CP plan hypothesis.

Proposition 5.2 Let $\langle\Phi, \prec\rangle$ be a quasi order set of the plan hypotheses, than there exists only one partitioning, $\pi$ of $\Phi$ such that each block of $\pi$ has only one least element, called the root plan hypothesis. This least element is always of the new plan hypothesis (NP) type. 
Proof. An NP plan hypothesis has no parents, but is comparable with its descending CP plan bypotheses of its block. So the NP plan hypothesis must be the least element of the block. Two NP plan hypotheses are not comparable, but the elements of a block are comparable, so there only can be one NP hypothesis in each block.

In a tracking system a similar partitioning can be defined. As with the tracking application, the characteristics of the partitioning are applied in the supporting functions like those of the plan hypothesis management in the tracking application, but also the parenthood relation is of importance in the calculation of the $k$-best assignment hypotheses in the case of data fusion.

One can also define a more restricted parenthood relation, the direct parenthood relation, that does not have any transitive elements. In this relation a plan hypothesis is only related to its parent of the previous scan. For this state the following proposition:

Proposition 5.3 The direct parenthood relation forms only one tree for each block of partition $\pi$, with the NP plan hypothesis as root and the CP plan hypotheses as nodes. The edges are determined by the direct parenthood relation.

Proof. A block only has one NP hypothesis, i.e. least element. A plan hypothesis has only one direct parent plan hypothesis. Hence the relation induces a tree.

In plan recognition with deterministic observations, the plan hypotheses remain identical with new observations, so each CP plan hypothesis is identical to its originating NP plan. This is not the case in plan recognition with probabilistic observations and parameter estimation for the plan hypotheses. Indeed, in this case, all plan hypotheses will be different. Remember that each block of the partition contained a tree induced by direct parenthood relations. A path between the basic NP plan hypothesis and any CP hypothesis exists and this path corresponds to a sequence of plan hypotheses. This sequence corresponds uniquely with a sequence of observations on which the plan hypotheses are based. Now introduce a well known theorem for trees:

Theorem 5.1 Let $T$ be a tree with root $r$ and let $a$ be any node of $T$. Then there is a unique path from $r$ to $a$ and visa versa. $\square$ 
And then state the following proposition regarding a sequence's uniqueness.

Proposition 5.4 An observation sequence leading to a plan hypothesis is unique.

Proof. The direct parenthood relation induces a tree with as root the NP hypothesis of the block of $\phi_{k}$. The sequence corresponds with a path from a node to the root of the tree. Theorem 5.1 states that a tree's paths are unique.

Now, if one assumes that a parameter estimator for plan hypotheses gives a unique result for a particular sequence of observations, then state the following proposition.

Proposition 5.5 No plan hypothesis of a probabilistic observation plan recognizer with plan hypothesis parameter estimation equals an other plan hypothesis.

Proof. Each path from the NP plan hypothesis to a CP plan is unique. So also the sequence of observations of the path is unique. The assumption was that the result of a parameter estimator is uniquely determined by its sequence of observations, hence the parameter estimator yields different results for each different plan hypothesis.

The path of plan hypotheses is uniquely identified by it's NP plan hypothesis. Remember that the life of a plan hypothesis is as long as its object is in the coverage. Only the estimated parameters have to be updated, but not the logical expression of the NP plan. So if the NPI plan, that helicopter $H 1$ attacks tank unit $T K 5$, was $N P 1=\operatorname{Attack}(H 1, T K 5, s v 1)$, where $s v 1$ is the initial state vector of $H 1$ and was based on the first observation, ol, then for the $n^{\text {th }}$ observation the plan looks like CP1n $=\operatorname{Atack}(H 1, T K 5$, svn $)$, where sun is the $n^{\text {th }}$ state vector. So the main proposition is still that helicopter $H 1$ attacks tank unit $T K 5$.

This chapter finished the formal description of Tactical Plan Recognition. The next step in the analysis, is to define tasks and their data-flows of a Tactical Plan Recognizer. This is done in the next chapter. It starts with a Generic Task Model for Identified observations. Then it will be extended to our Tactical Plan Recognition problem. 


\subsection{Soundness and completeness}

Given the formal descriptions of the output of a Tactical Plan Recognizer, we can define the soundness and completeness properties for Tactical Plan Recognition algorithms. Define soundness as:

Definition 5.6 A Tactical Plan Recognition algorithm is sound when every solution it produces is indeed a solution of the plan recognition problem posed by its input set of observations. Each solution satisfies definition 5.1 for a single plan recognition problem and definition 5.2 for multiple independent plan recognition problem.

The soundness requirements relate an observed action with the plan hypotheses. The plan hypotheses are sound when they, partially, contain the abserved action. In that case the observed action is entailed by the hypothetical plan and the background knowledge and the explanation property of the plan hypothesis is true. In fact the plan hypothesis must contain also an action of any other observation it is consistent with:

Assumption 5.1 A plan hypothesis based on an observation o or is consistent with an observation $o$ should, at least, contain an action that will unify with the observed action $o$.

For example: if Fly(target, $x, y)$ was observed, a suitable plan would contain an action like Fly (Zwean, Amsterdam, Heathrow).

Define completeness as follows:

Definition 5.7 A Tactical Plan Recognition algorithm is complete, when it generates all possible sound plan hypotheses, given a set of observations and the background knowledge.

The limiting sentence 'given a $\cdots$ and the background knowledge' applies; due to Azarewicz's closed world assumptions 4.1 and 4.2 .

\subsection{Optimal Tactical Plan Recognition}

One of the goals of the problem statement was to develop an optimal Tactical Plan Recognition algorithm. Having looked at the character of the Tactical Plan Recognition problem in the previous sections, we can now elaborate 
the optimality requirement. An algorithm should optimal in the sense that it outputs the k-best assignment hypotheses after processing a batch of observations belonging to one scan. If one adopts Reid's formula (formula 5.3) then the optimal solution is in fact the assignment hypothesis with the highest probability.

The probability is, however, only approximated. This because the correlation probability of an observation with a plan hypothesis is based on an observation that entails a measurement error and because the plan hypothesis' kinematic parameters are only estimated.

\subsubsection{The optimization problem}

The optimization problem is to find $k$ assignments $\Psi_{h}$ that have the highest assignment probability $P\left(\Omega_{g}^{k-1}, \Psi_{h} \mid \mathcal{O}\right)$. All assignments are generated by the hypothesis tree 'HypTree' at hand. More formally stated:

$$
\begin{aligned}
k-\text { best } & P\left(\Omega_{g}^{k-1}, \Psi_{h} \mid \mathcal{O}\right) \\
\text { s.t. } & \Psi_{h} \in \text { HypTree }
\end{aligned}
$$

A more detailed mathematical model is given by an assignment problem definition. This type of formalization was done by Krüger [45] and is given below. Here $i \in[1, m]$ (where $m=M_{K}$ ) is an iterator that runs over the set of observations of a scan and $j \in[1, n]$ (where $n=N_{T C T}+N_{C P}+N_{F A}+$ $N_{N P}, N_{C P}$ is the number of correlated plans that can be continued by a new observation by another observer within the same scan) is an iterator that runs of the set of plan bypotheses in a scan:

$$
\begin{array}{rll}
k \text {-best } & \prod_{i=1}^{m} \prod_{j=1}^{n} c_{i j} x_{i j} & \\
\text { s.t. } & \sum_{j=1}^{n} x_{i j}=1 & \text { for } i=1,2, \cdots, m \\
& \sum_{i=1}^{m} x_{i j}<1 & \text { for } j=1,2, \cdots, n \\
& x_{i_{c} j_{c}}-x_{i_{p} j_{j}} \leq 0 & \text { for all child-parent pairs }\left(x_{i_{c} j_{c}}, x_{i_{p} j_{p}}\right) \\
& x_{i j} \in\{0,1\} & \text { for } i=1, \cdots, m ; j=1, \cdots, n
\end{array}
$$

The first two constraints: $\sum_{j=1}^{n} x_{i j}=1$ and $\sum_{i=1}^{m} x_{i j}<1$, combined with the $x_{i j} \in\{0,1\}$ requirement, are generally found in a rectangular assignment problem. The third constraint: $x_{i_{c} j_{c}}-x_{i_{p} j_{p}} \leq 0$ are additional 'parenthood' 
constraints that occur in a sensor data fusion problem and are introduced in Mulder, et. al. [62]. The constraint ensures that no child plan hypothesis will exist in an assignment when its parent is not a part of the assignment.

The elements $c_{i j}$ in the formulas above are determined by the type of the $j^{\text {th }}$ plan hypothesis. Here there are the following cases:

- if the plan hypothesis is an already existing plan hypothesis, then $c_{1 j}=$ $P_{D} g_{x y i j} /\left(1-P_{D}\right)$, where $P_{D}$ is the observation's sensor detection probability and $g_{i j}$ is given by the correlation likelihood of observation $\dot{i}$ with plan hypothesis $j$.

- if the plan hypothesis is a false alarm: $c_{i j}=\beta_{F A}$

- if the plan hypothesis is a new plan hypothesis: $c_{i j}=\beta_{N T}$.

For the correlation with an already existing plan, $c_{i j}=P_{D} g_{x y} /\left(1-P_{D}\right)$. So it is always divided by $\left(1-P_{D}\right)$. So for $N_{D T}$ detected targets there is a division by $\left(1-P_{D}\right)^{N_{D T}}$. This is done in anticipation of the normalization of the probability as is done in the reduce function. Here the probability is multiplied by $\left(1-P_{D}\right)^{N_{T G T}}$. So after normalization, the part of the probability that is due to the old targets being undetected or detected is: $P_{D}^{N_{D T}}\left(1-P_{D}\right)^{N_{T G T}-N_{D T}}$. This approach is useful in the application of $\mathrm{k}$-best linear assignment problem algorithms as described in Verveld [91]. To take the parenthood constraints into account, the branch and bound technique can be applied efficiently, see Krïger [45] and Sibma [79].

\subsubsection{Optimal kinematic estimation}

As mentioned before, the calculation of the correlation likelihood $g_{x y}$ necessitated an optimal prediction of the planned position. Therefore an optimal estimation of kinematic parameters is required. In Reid's model, a stochastic process model of a dynamic system is devised for the target's kinematics. Going back to the basics of estimation theory, consider the general stochastic process equation:

$$
x_{t}=\mu\left(x_{t}, t\right)+\sigma\left(x_{t}, t\right) w_{t}
$$

where $t \geq 0$ and $x_{t}$ is a state vector and $w_{t}$ represents noise. $\mu$ is a transition functional matrix and $\sigma$ is a disturbance function. The stochastic process is 
observed, resulting into a set of measurements that are related to the state vector by the following measurement equation:

$$
z_{t}=h\left(x_{t}, t\right)+v_{t}
$$

where $z_{t}$ is a measurement vector, $h\left(x_{t}, t\right)$ is a measurement functional matrix and $v_{t}$ represents the measurement noise. $h$ is the measurement functional matrix.

The estimation algorithm will try to estimate the actual values of the state vector by means of the measurement vector. Of course the estimated state vector, $\hat{x}_{t}$, will have an error, $x_{t}-\hat{x}_{t}$. An optimal estimation algorithm will try to minimize the error.

If the dynamic system is linear and the observation and process noise are Gaussian, the optimal estimator is given by the Kalman filter. This filter is optimal in the sense that is minimizes the diagonal elements of the updated state vector"s covariance matrix.

\subsubsection{Optimality over several scans}

The model of equation 5.7 concerns the k-best assignments for one scan, based on the optimal assignment of the previous scan. The hypotheses are reduced each scan to one optimal, so one does not search to an optimal assignment over all scans. What we ideally want is a method that gives the optimal assignment over all scans, the current and the previous ones.

Though the optimality over all scans can be attained by means of adding of parenthood constraints for the detections of tracks that existed in the previous scan, practice has shown that maintaining $k$-best assigmment hypotheses after each scan and searching for the $k$-best assignment hypothesis of the current scan gives good results.

Here also operations research algorithms, based on Murty's k-best algorithm [67], can be used. In Sibma's master's thesis a description of such an application is given for a sensor data fusion problem [79]. 


\section{Chapter 6}

\section{Generic Task Models for Plan Recognition}

In the previous sections, the Tactical Plan Recognition's input and output data were described. Also formal relations between input and output data were established. The next task is to develop computer algorithms that can perform the processing between input and output data. The first step for this development is the task and information analysis. This results in a Generic Task Model.

As planning and plan recognition are closely related, we first analyse the processes of reactive planning and distributed planning. It is useful to analyse these types of planning further, because they are found in the military world. After this we will examine the two plan recognition problems. First the plan recognizer for identified observations and then the plan recognizer for unidentified observations. The final result are two Generic Task Models.

\subsection{Reactive planning}

A real world planner has to cope with the following uncertainties. First, uncertainties about events, the state of any object may differ from expected states (because of unexpected events), and the number of objects and goals may vary unpredictably. Second, uncertainties about actions: this may involve operator failure: activity is wrongly executed, or there is incomplete knowledge about actions, e.g. enemy's actions or because of a wrong and/or incomplete model of actions (frame problem, qualification problem, rannification problem, for a 
engineering oriented discussion about these problems see [47]) or of planning objects. They have to be dealt with when they cause unexpected conflicts in a plan or when a plan has to be improved. To deal with these problems two methods are applied:

1. Reactive planning, first described by Wilkins, $[95,70]$. Monitor relevant aspects of the world during plan execution to recognize unexpected events and conflicts or opportunities for plan improvement. If one of these occurs, adapt the plan(-part) so that it is conflict-free again or improve the plan when the new state promotes it.

2. If the models are not apt any more, improve or make conflict-free, the model of actions, see Georgeff and Lansky, [32, 33], or the model of the planning objects. Generally an instantiated model is updated run-time to specific situations, after which new conflict-free and/or improved plan can be composed, based on the improved models.

\subsection{Distributed planning and plan recognition}

In distributed planning or distributed plan recognition a planning problem is solved by a collection of Joosely coupled cooperating agents. Cooperating implies here that they do not oppose each other. Loosely coupled means that the agents spend more time on their own processing than on communicating with each other.

The planning problem is partitioned in a number of relatively independent sub-problems. A set of these sub-problems is solved by an agent. For partitioning a problem there are two basic methods: functional partitioning and data partitioning. In a functional partitioning some functions are implemented by an agent which handles all plamning objects. In data partitioning the set of planning objects is divided into clusters of objects which are tightly related (with regard to the planning) to other objects in the same cluster, but loosely related to objects of other clusters. For each cluster an agent devises a local plan. The set of local plans constitutes the global plan.

A (rather complex) Generic Task Model for reactive distributed planning is given in Mulder et. al. [64]. 


\subsection{Analysis aspects}

One can discuss a problem by restricting oneself subsequently to different aspects of the problem to be solved. We shall discuss some interesting aspects and associated problems of distributed reactive planning. We subsequently develop a Generic Task Model for each problem. The aspects are 1) domain, 2) control and 3) coordination. The associated problems are:

- the domain problem is the problem for which the system is devised. E.g. operational plamning (an instance of reactive planning) and tactical airborne plan recognition (an instance of plan recognition).

- the control problem, defined by Hayes-Roth [36]: dynamically decide when which (domain/control/coordination) chunk of knowledge (Knowledge Source) of one agent should be activated in a particular problem solving situation. In more complex cases the control problem itself is a reactive planning problem! However, not all knowledge needs to be activated explicitly by the control component and the granularity of the knowledge sources should be apt to a specific problem.

- the coordination problem, first described by Lesser [49]: attuning the planning and plan recognition activities of several agents in order to attain global conflict free and globally (sufficiently) optimal solution. This is done by exchanging partial plans and auxiliary information at a suitable granularity.

\subsection{Generic Task Models}

In this section the Generic Task Models for two cases of plan recognition are presented: plan recognition with identified observations, the simpler case, and then plan recognition with unidentified observations, the more complex case.

\subsubsection{Plan Recognition with identified observations: the do- main problem}

Generic tasks of an identified observation plan recognizer are displayed in figure 6.1, pg. 112 from Mullder [56]. Not all tasks are meaningful for all reactive plan recognition problems, for example, if the number of plan-hypotheses is 


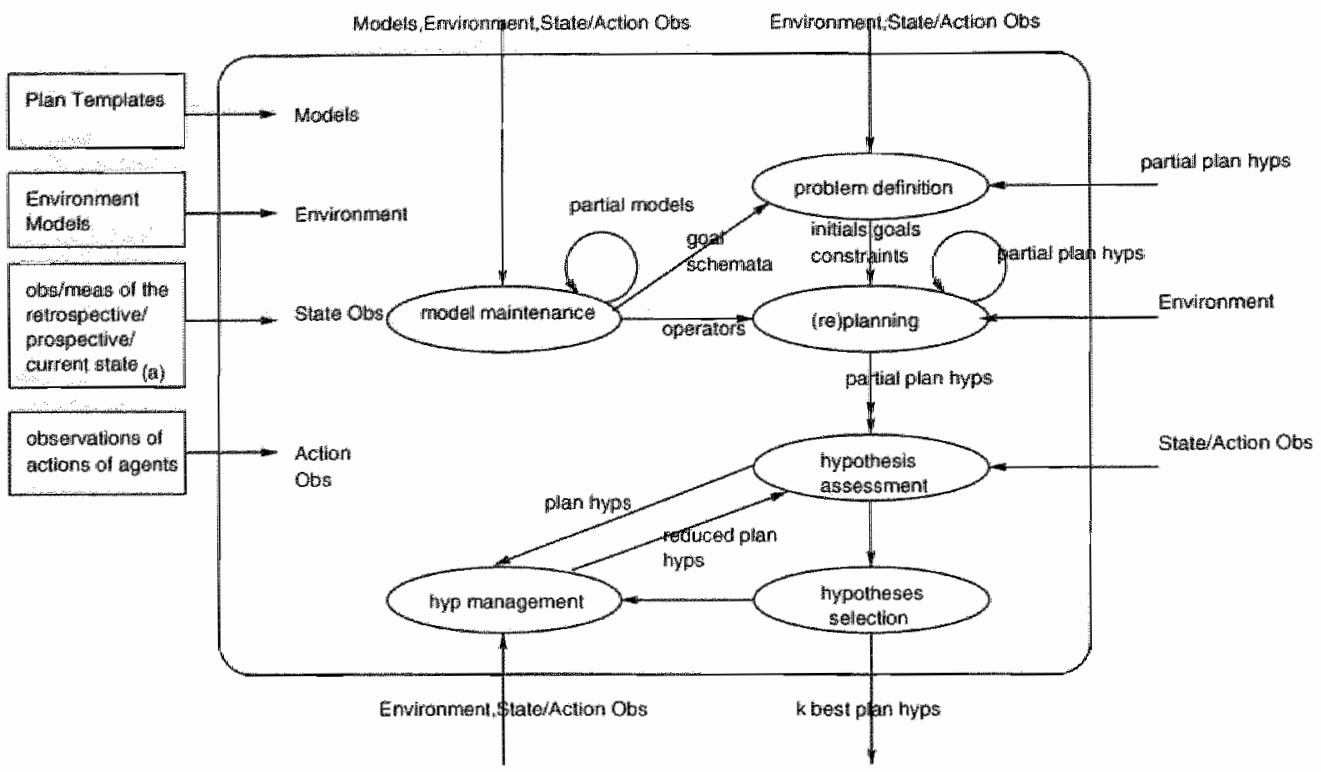

Figure 6.1: A Generic Task Model for non-hierarchal/identified observation plan recognition

limited, then hypotheses management may not be required. The generic tasks are listed below.

- Model maintenance (for a definition of the term maintenance see [87, pg. 327]): ensuring that the models of the world behave (continues to behave) as well as possible according to the reference model: the real world. Dynamic, and clefault or uncertain aspects of the model have to be updated regularly.

- Problem definition ${ }^{1}$ : during problem definition, possible goals of newly observed objects are recognized and all information to generate hypothetical plans for the goals is made explicit. For example, given the objects and the initials, constraints and goals are recognized.

- (Re)planning: a partial plan is an arrangement (for the definition of the term arrangement see [37]) or system synthesis (for the definition

\footnotetext{
${ }^{1}$ This name is not very suited to denote a task, as it does not contain a verb. However, it has already been used in a publications of Mulder and Braspenning $[56,61]$ and, therefore, is retained here.
} 
of the term synthesis see [87, pg. 327]) of activities. Given a certain context and a set of goals, arrangements of activities are developed. These arrangements satisfy constraints and are sufficiently optimal.

- Hypotheses management has two tasks: first, to remove plan hypotheses which are not consistent any more with the current state of the world. And secondly, if plans and models are combined, the number of hypotheses may increase significantly. Then it may be necessary to restrict the number of plan hypotheses considered for plan-state assessment. Methods for restricting the number of hypotheses are given in the table below.

\begin{tabular}{|l|l|}
\hline method & description \\
\hline \hline pruning & cutting branches \\
\hline combining & binding similar branches together \\
\hline wrindowing (gating) & $\begin{array}{l}\text { data validation (prevent generation of hypothe- } \\
\text { ses based upon less likely data) }\end{array}$ \\
\hline clustering & data partitioning \\
\hline
\end{tabular}

- Hypotheses assessment: (assessment is defined in [87, pg. 294]): assess the belief in the plan hypotheses based on the belief of the supporting observations. These can be directly correlated to a hypothetical plan, as they share the same identification. It depends of course on the type of reasoning about uncertainty, how the task is implemented.

- Hypotheses selection: select the $\mathrm{k}$ best hypotheses to be out-putted.

So in the Generic Task Models possible, hypothetical plans which might explain the observations are created. The direct support of a plan hypothesis by a set of observations, belonging to the object(s) of the hypothetical plan, is calculated and the best hypothetical plans are selected and out-putted.

\subsubsection{Plan Recognition with unidentified observations: the domain problem}

For this class of plan recognizers consider that in the case of identified observations, the objects involved in the observations may execute only one single plan or multiple dependent or independent plans. In the case of multiple plans, the assignment of an observation to a subset of the plan hypotheses that originates of one target, is readily done by means of the identity of the objects in the plan hypotheses and the identity of the objects in the observations. 


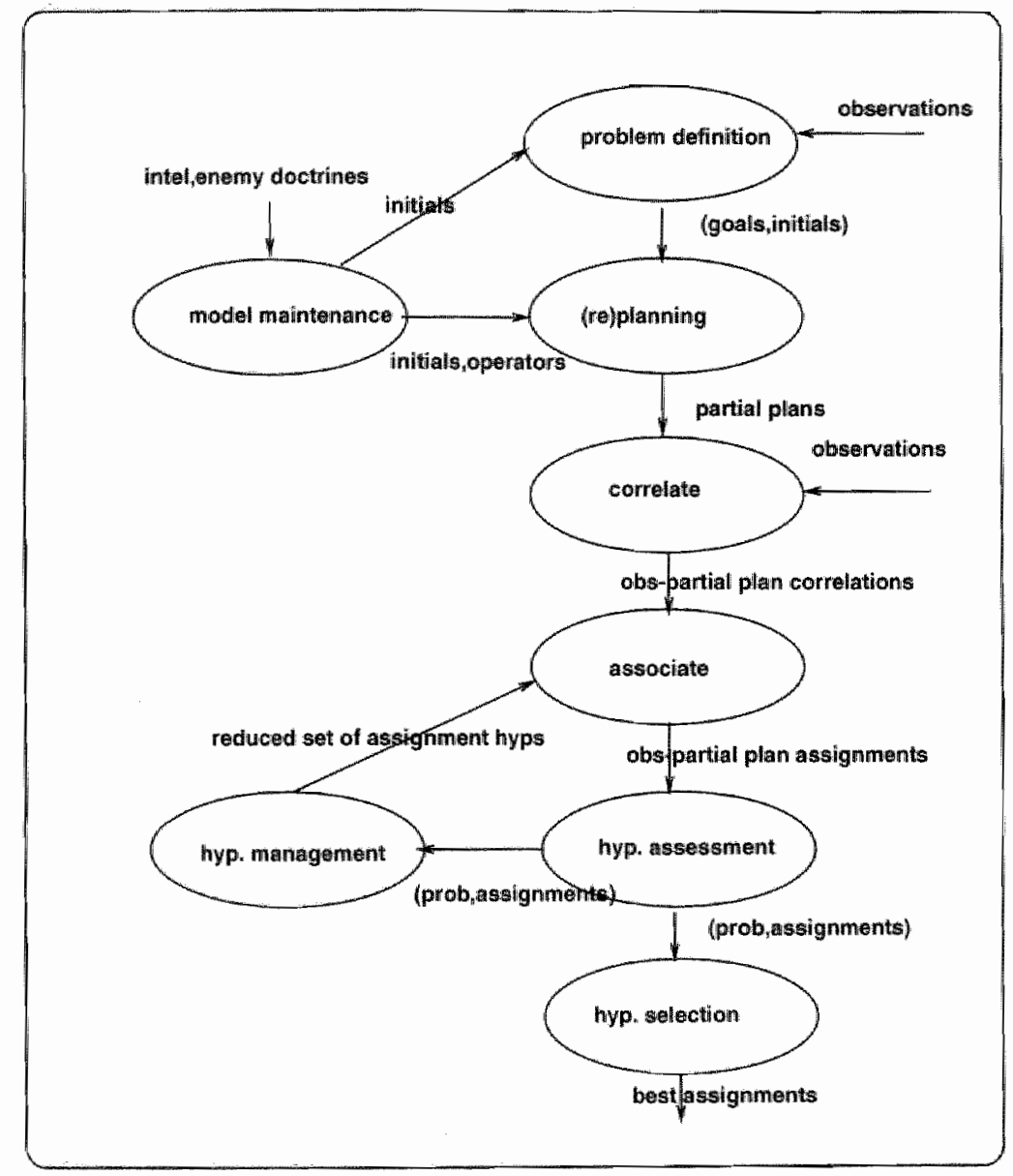

Figure 6.2: A Generic Task Model of non-hierarchical/unidentified observations plan recognition. 
The Generic Task Model of an unidentified observation plan recognizer is displayed in figure 6.2, page 114 and is from Mulder and Braspenning [61]. Some of the generic tasks are identical to the Generic Task Model for identified observations and are already explained there. Also note that the origin of some of the data flows, as shown in figure 6.1 and such as state observations and action observations, are omitted in the Ceneric Task Model of figure 6.2. A description of the tasks in the figure is given below.

- Model maintenance [87, pg. 327]: ensuring that the models of the world behave (continues to behave) as well as possible according to the reference model: the real world. Dynamic, and default or uncertain aspects of the model have to be updated regularly.

- Problem definition: during problem definition, possible goals of newly observed objects are recognized and all information to generate hypothetical plans for the goals is made explicit.

- (Re)planning: a partial plan is an arrangement (for a definition of arrangement see [37]) or system synthesis (for a definition of synthesis see $[87, \mathrm{pg} .327])$ of activities. Given a certain context and a set of goals, arrangements of activities are developed. These arrangements satisfy constraints and are sufficiently optimal.

- Correlate: recognize, possible, hypothetical plan - observation associations, i.e. recognize which observations might originate from the objects that execute a particular hypothetical plan. The observations might refer to an action. In that case they can be correlated directly to a hypothetical plan. Other observations concern a state of the outside world at a particular time. In that case, first the predicted state, in so far it is related to the observation, is calculated. This is done according to the plan hypothesis to be associated with the observation. It simply entails finding the state of the plan at the prediction time.

- Hypotheses association: generate assignment hypotheses of observations to old plan hypotheses. Also generate assignment hypotheses to new hypothetical plans that are generated for the observations.

- Hypothesis assessment: (assessment is defined in [87, pg. 294]): assess the belief of an assignment hypothesis, representing a possible assignment of observations to a set of hypothetical plans. For each observa- 
tion that enters the system it is checked whether it is consistent with the existing hypotheses. If an existing hypothesis is not consistent with the new hypothesis, it is removed from the set of hypotheses. Furthermore a ranking of the hypotheses can be added or modified in view of the new observation.

- Hypotheses management has two tasks: first, to remove plan hypotheses which are not consistent any more with the current state of the world. And secondly, if plans and models are combined, the number of hypotheses may increase significantly. Then it may be necessary to restrict the number of plan hypotheses considered for plan-state assessment. Methods for restricting the number of hypotheses are given in the table below.

\begin{tabular}{|l|l|}
\hline method & description \\
\hline \hline pruning & cutting branches \\
\hline combining & binding similar branches together \\
\hline windowing (gating) & $\begin{array}{l}\text { data validation (prevent generation of hypothe- } \\
\text { ses based upon less likely data) }\end{array}$ \\
\hline clustering & data partitioning \\
\hline
\end{tabular}

This section finishes the analysis of "traditional" plan recognition and Tactical Plan Recognition. Formal requirements were stated and the tasks and sub-tasks of plan recognizers were defined. The following part of this thesis concerns the design of algorithms that implement the Generic Task Models. Here some algorithms for plan recognizers will be presented. Some formal properties, such as soundness and completeness will be examined for each algorithm. 


\section{Summary of part II}

In this part on the analysis of Tactical Plan Recognition we have shown that Tactical Plan Recognition differs significantly from classical plan recognition. In classical plan recognition the plan recognizer has as input certain, identified observations of actions. Whereas, in Tactical Plan Recognition, the observations are uncertain in various ways. The uncertainties of the observations have been listed here and formal representations of the uncertainties have been described. The most important uncertainty is that identity of the observed objects is often unknown.

Then the text focusses on the formal definition of Tactical Plan Recognition. To accommodate for Tactical Plan Recognition, the formal properties for traditional plan recognition have been extended. The formal definition of traditional plan recognition, that was used here, was based on classical abduction.

The abduction-based definition of traditional plan recognition relates the input of the plan recognizer, i.e. background lnowledge and observations, with the output of the recognizer, i.e. the plan hypotheses. They are related by the consistency and explanation property. Due to these formal requirements, the acceptable plan hypotheses are restricted; not any plan hypothesis will do.

In the examples 2.1 and 2.3 of section 2.3 of part I, it was shown that a tactical plan recognizer has to deal with unidentified observations, and therefore shall generate assignment hypotheses. An assignment hypothesis links an unidentified observation with a plan hypothesis. To give a formal definition of tactical plan recognition, the assignment function is introduced and now an equivalence class of observation is required to be consistent with the assigned plan hypotheses of the observation. Similarly, the assigned plan hypotheses of an observation must explain the observation.

When the formal definitions are available, they are then illustrated by a large number of examples. These are based on the civil aviation and the army 
examples 2.1 and 2.3. The examples start with a simple case of identified observations and their complexity is gradually increased to the unidentified observations case. For each example, the formal properties have been checked. However, there is no algorithm presented that can automatically generate the plan hypotheses and assignment hypotheses of the examples.

In the case of deterministic observations and background knowledge, one can only state a number of hypotheses, but there is no formal method to rank them. In the case of probabilistic knowledge one can apply some general method for reasoning under uncertainty, such as Bayesian nets or the Dempster-Shafer combination rule. In the particular case of probabilistic and unidentified observations, one can calculate the probability of the assignment hypotheses, by adapting Reid's MHT formula. Thus the hypotheses can be ranked and the k-best can be selected.

This chapter ends with a description of some general properties of a Tactical Plan Recognizer; some properties of the hypotheses, a formal definition of the soundness and completeness and two definitions for its optimality.

After the formal part, we start with the design of algorithms that can do plan recognition. The first stage of this development consists of the development of Generic Task Models for Tactical Plan Recognition. Again, the text starts with the simpler problem of plan recognition based on identified observations and then handle the case with unidentified observations. In fact many of the functions of the identified observation plan recognizer are found again in the unidentified plan recognizer, but there are some new tasks that generate and asses assignment hypotheses. The Generic Task Model is the basis for the algorithm design in the next part.

So we can conclude that, in part II, it is shown that the definitions of classical abduction can be adapted usefully to the Tactical Plan Recognition problem. The application of the formal definition has been shown in a large number of examples. The first analysis of the problem resulted in a Generic Task Model for Tactical Plan Recognition. 


\section{Part III}

\section{Design of Tactical Plan \\ Recognition algorithms}





\section{Chapter 7}

\section{Design of the tasks of a Tactical Plan Recognizer}

In this chapter, a design of the some of the larger tasks of the Generic Task Model of Tactical Plan Recognition is proposed. We start with the design of the problem definition task, followed by that of the planning task. The chapter ends with some kinematic state estimation algorithms for the model maintenance task.

After designing a task, we would like to make formal statements about the design: its soundness and completeness. To make statements about the completeness of a plan recognizer concerns two parts: 1) the completeness of the knowledge base and 2) the completeness of the algorithms or inference mechanisms. It is not possible to make a definite statement of the completeness of the modelled doctrines and background knowledge in the knowledge base. This fact would halt any attempt to make a formal statement about the plan recognition system. Here the closed world assumptions give a way out. They 'make' the knowledge base complete. So we are only left with the task to examine the inference methods and algorithms applied.

\subsection{Examples recalled}

In section 2.3 , page 28 , a civil aviation example and an army example of plan recognition were given. The examples will be used to show the workings of the goal recognition and planning algorithms, so they are recalled here: 
Example 7.1 Flight KL174 and KL175: In a first example consider two aeroplanes S ("Swean") and Z ("Zwaluw") which can be used for two routes: KL174 from $\mathrm{H}$ (Heathrow) to $F$ (Frankfurt) and KL175 from Frankfurt to Heathrow. Both flights stop in A (Amsterdam). This results in four possible plans:

Flight $(K L 174, S)$, Flight $(K L 174, Z)$, Flight $(K L 175, S)$ and Flight $(K L 175, Z)$. The background knowledge $\Gamma$ contains the following formulas:

$$
\begin{aligned}
& \forall x F l i g h t(K L 174, x) \Leftrightarrow F l y(H, A, x) \wedge S \operatorname{Stop}(A, x) \wedge F l y(A, F, x) \\
& \forall x F \operatorname{light}(K L 175, x) \Leftrightarrow F l y(F, A, x) \wedge \operatorname{Stop}(A, x) \wedge F l y(A, H, x)
\end{aligned}
$$

These formulas are 'plan templates' from which actual plan hypotheses can be derived.

A consistent sequence of observations is: $[F l y(H, A, S), S$ top $(A, S)]$ saying that $\mathrm{S}$ has been observed flying from Heathrow to Amsterdam and after that has been seen at Amsterdam. An inconsistent sequence of observations is: $[S t o p(A, S), F l y(H, A, S)]$.

Next, consider the, somewhat more difficult, army example. The situation is graphically displayed in figure 2.6 (page 35 ).

Example 7.2 Army Tactical Plan Recognition example: consider an attack helicopter $\mathrm{H} 1$ and a start base $\mathrm{U} 1$, a target $\mathrm{U} 5$ and a return base FARP. The background knowledge now is: $\{F$ oe $(A B 1), F$ oe $(F A R P)$, Friend $(T K 4)$, Friend $(T K 5)$, Friend $(T K 6)\}$, combined with rules about possible plans: $\forall x, a, b, c \operatorname{Attack}(x, a, b, c) \Leftrightarrow F l y(x, a, b) \wedge \operatorname{Attack}(x, b) \wedge F l y(x, b, c)$ also it is required for such an attack that the following facts about the allegiance of objects of the variables $x, a, b, c$ are true: $F$ oe $(x) \wedge F$ oe $(a) \wedge F r i e n d(b) \wedge F$ oe $(c)$.

A consistent sequence of observations is: $[F l y(H 1, A B 1, T K 5), F l y(H 1, U 5, F A R P]$ saying that $\mathrm{H} 1$ has been observed flying from unit 1 to unit 5 and after that has been seen at flying from unit 5 to the enemy forward air refuelling point. An inconsistent sequence of observations is: $[$ Attack $(H 1, T K 5), F l y(H 1, A B 1, T K 5)]$.

Another passible plan is that the enemy only wants to reconnoiter an enemy object: $\forall x, a, b, c \Leftrightarrow \operatorname{Recce}(x, a, b, c) \Leftrightarrow F l y(x, a, b) \wedge$ TakePhoto $(x, b) \wedge$
$F l y(x, b, c) \square$ 
For the situations above, the goals or intend of the observed agents are given below.

Example 7.3 Refer to example 7.1, Flight KL174 and KL175: in the civil aviation example the goal of flight KL174 is to transport people from Heatlurow to Frankfurt and of flight KL175 to transport people from Frankfurt to Heathrow by the airplane 'Swean' executing Flight KL174, so At(Swean, Heathrow) $\square$

Example 7.4 Refer to example 7.2, the army example: in the army example the goal for the attack plan is to destroy a friendly unit, e.g. Destroyed(H1, $A B 1, U 5, F A R P)$. The goal for the reconnoiter plan is to know details of the friendly unit.

Now that we have stated the examples to be used in the thesis we can start with examining the goal recognition systems for these examples.

\subsection{The design of the Problem Definition task}

In this section, algorithms for the problem definition task ${ }^{1}$ of the Generic Task Model are discussed. The problem definition and the planning tasks cooperate in generating all possible plans given a sequence of observations. The first step of the problem definition task is to recognize all the goals that the observed agents may have. The second step of this task is to collect all background information that is relevant to the recognized goals. The collected background information and recognized goals are input for the planning function that generates all plans that may satisfy the recognized goals. The interaction between the two tasks is depicted in figure 7.1.

Some formal requirements on the problem definition and planning algorithms have to be satisfied: soundness and completeness. If the algorithms are sound, the output of the functions are solutions of the input problems. If the algorithms are complete, indeed all possible solutions to their input problems are generated. However, the completeness is only with respect to the available background knowledge of the algorithms.

\footnotetext{
${ }^{1}$ Again, it is noted that the term "problem definition" is a rather unfortunate name for the task (it does not contain a verb), but as it has been used in previous publications, it is retained in this document. A better name is "recognize goals and collect background information".
} 


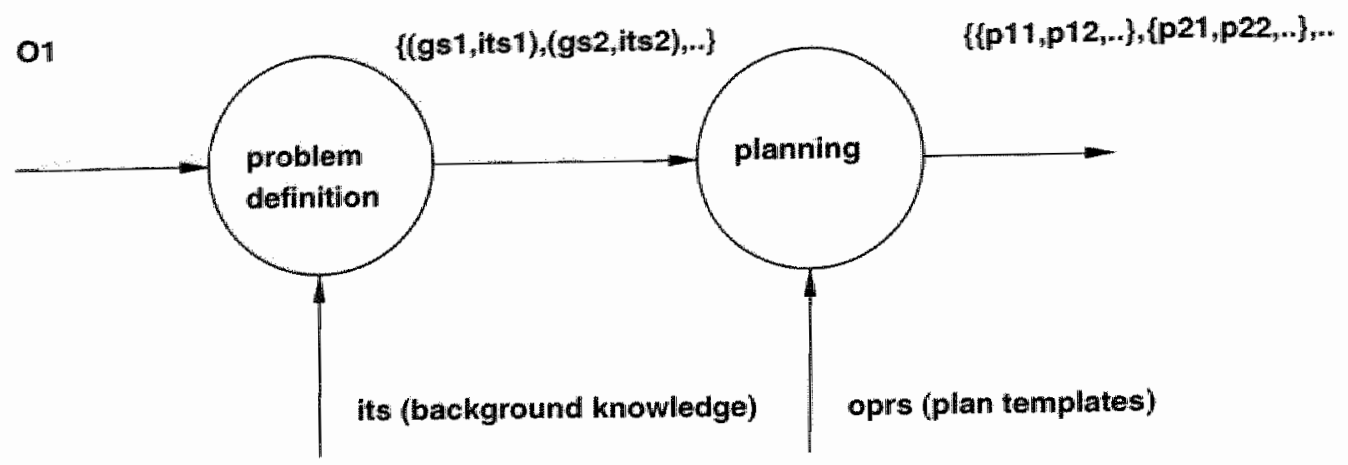

Figure 7.1: The interaction between problem definition and planning. The input is an observation, the problem definition task generates a set of pairs of goals , $g s 1, g s 2, \cdots$, and background information ,its 1 , its $2, \cdots$, for the observation. These are input to the planning, that generates sets hypothetical plans, $\{\{p x 1, p x 2, \cdots\}$ for each pair (gsx, its $x)$.

Now summarize the workings of the problem definition task. We only start with a set of observations and background knowledge and then we have to find hypothetical goals of the observed agents and the relevant background knowledge. The problem definition task has two main functions: 1) goal recognition and 2) compiling relevant background information. We will only discuss the goal recognition task, the second task is a simple query on the background knowledge.

\subsubsection{A Goal recognition knowledge base}

The knowledge base of the goal recognition consists of a number of implications. In our examples the application area involves a geographic situation and movements of agents and the conditions of the implications contain many geographic predicates and functions. For this a geographic information system (GIS) has to be applied. Consider some rules for the first example.

Example 7.5 Flight KL174 and KL175: here the knowledge base of the goal recognizer consists of the following rules: 


\begin{tabular}{lll} 
Rule & observations & goals \\
\hline R1: & $\forall x F l y(H, A, x)$ & $\Rightarrow A t(x, F)$ \\
R2: & $\forall x \operatorname{Stop}(A, x)$ & $\Rightarrow A t(x, F)$ \\
R3: & $\forall x F l y(A, F, x)$ & $\Rightarrow A t(x, F)$ \\
R4: & $\forall x F l y(F, A, x)$ & $\Rightarrow A t(x, H)$ \\
R5: & $\forall x \operatorname{Stop}(A, x)$ & $\Rightarrow A t(x, H)$ \\
R6: & $\forall x F l y(A, H, x)$ & $\Rightarrow A t(x, H)$ \\
\hline
\end{tabular}

Where $A t(x, y)$ indicates that target $x$ is at location $y, A$ is Amsterdam, $F$ is Frankfurt and $H$ is Heathrow. The rules link all possible observations with all possible goals. The recognized goals are only hypothetical. There can be multiple hypothetical goals in the database, even though they concern the same observed object. $\square$

In the example above, the observations directly concern steps of plans. Also one observation leads directly to a goal. In practice, if an aeroplane is observed flying in some direction, one does not know the starting point and the destination of the leg. In the case of human intelligence the observation only states the position and the heading of the plane. From these more uncertain observations the goal recognizer has to do its job. The goal recognizer then has to apply geographic knowledge. This is considered in the army example 7.2 , where the knowledge base of the goal recognizer is:

Example 7.6 Extended army Tactical Plan Recognition example 7.2: the set of rules here are:

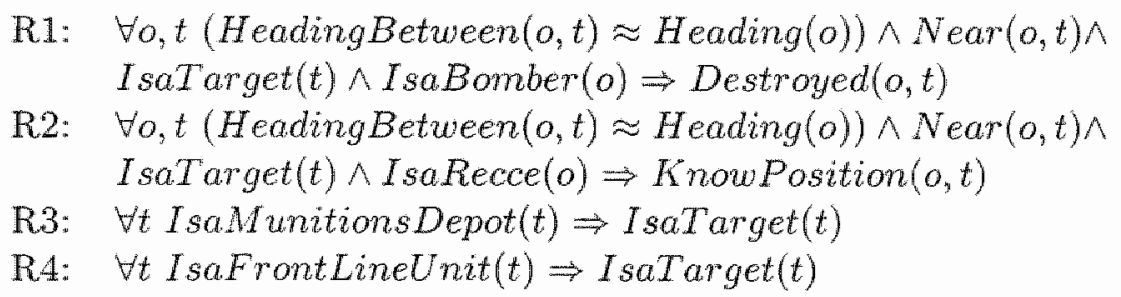

Where $o$ is the object of an observation and $t$ is a target. The possible targets are listed in the initials. Heading indicates a function that returns the direction of the flight, of the object, e.g. north-west, north, etc. HeadingBetween indicates the direction of a straight line from the object of an observation and a target. In the case that the observed object is a bomber (indicated by 
the predicate IsaBomber) the goal is obviously to destroy the target. In the case that the observed object is a reconnaissance aircraft (indicated by the predicate IsaRecce), the goal is to know the position of the target.

The Geographic Information System (GIS) provides a number of the predicates in the conditions of the rules. In the example we find the following GIS functions: HeadingBetween, Heading, IsaFrontLineUnit and Near. Concerning the relation $\approx$ : it models some form of uncertainty, i.e. the difference in heading between the left part and the right part of the relation should be within a certain fixed range.

The rule base above requires an inference system that maintains a search of deductions, as long as any rule can be fired. This is the case in a forward chaining rule based system. In our example first one has to derive whether a friendly object is a target in rules R3 and R4 and then use their conclusions to fire rules $\mathrm{R} 1$ and $\mathrm{R} 2$.

\subsubsection{Soundness and completeness of a forward-chainer}

In line with the problem statement, we to discuss the soundness and completeness of the forward chaining inference method. Informally stated from Russel and Norvig [76]:

Inference is the process of deriving new sentences from old ones. We try to design sound inference processes that derive true conclusions given true premisses. An inference process is complete if it can derive all true conclusions from a set of premisses.

There are several inference methods that can be applied on the goal recognition knowledge bases. We will discuss the forward chaining method that is based on the generalized modus ponens inference rule ${ }^{2}$. Though generalized modus ponens is a sound method, it is incomplete. It is incomplete, because there are sentences, entailed by the knowledge base, that a modus ponens based inference mechanism camnot infer. The following example is taken from
Russel and Norvig [76]:

Example 7.7 Suppose we have the following knowledge base:

\footnotetext{
${ }^{2}$ Moclus ponens is a well known inference rule for propositional logic; the generalized form
such a rule for predicate logic.
} 


$$
\begin{aligned}
& \forall x P(x) \Rightarrow Q(x) \\
& \forall x \neg P(x) \Rightarrow R(x) \\
& \forall x Q(x) \Rightarrow S(x) \\
& \forall x R(x) \Rightarrow S(x)
\end{aligned}
$$

Suppose we want to be able to infer $S(A) . S(A)$ is true if $Q(A)$ or $R(A)$ is true, and one of these must be true, because either $\mathrm{P}(\mathrm{A})$ or $\neg P(A)$ is true. The problem here is that $\forall x \rightarrow P(x) \Rightarrow R(x)$ cannot be converted to a Horn clause: $C_{1} \wedge C_{2} \wedge \cdots \Rightarrow A$, where $C_{1}, C_{2}, \cdots$ and $A$ are non negated atoms. If $A$ is false, the clause is an integrity constraint (mutual exclusion of the atoms); if $A$ is an atom, it is called definite clause. A Horn form of the knowledge base simply is a requirement for applying generalized Modus ponens. $\square$

However, when the implications are Horn sentences, the forward chaining inference mechanism is complete.

Resolution is a more complex inference method, that does not require a. knowledge base of Horn sentences. However, for our purposes for the goal recognition, generalized Modus ponens suffices.

\subsubsection{Computational complexity of the forward-chainer}

The computational complexity is determined by the number of facts, $W$, in the forward-chainer's knowledge base, the number of precondition atoms in the rules, $C$, and the number of rules, $R$. The complexity is, best case, $O\left(\log _{2} R\right)$ and, worst case, $O\left(R W^{C}\right)$. The average case complexity of the most popular forward chaining algorithm, RETE, is given in Albert and Fages, [2, 1]. So the forward chaining inference mechanism is exponential with the complexity of the rules. Nevertheless efficient rule based systems can be devised for problems with a limited number of sentences. When the rules are compiled, even real time characteristics are attainable.

\subsection{The design of the Planning task}

In the examples, some planning functions were implemented by means of logical equivalences as templates and logical inferences as planning function. This approach suffices for simple plans but is able to model complex plans. For the generation of complex plans, specialized planning algorithms are required. Planning algorithms have long been a research topic in the field of computer science and artificial intelligence. 
The input of a traditional planning algorithm are goals and background knowledge. The background knowledge consists of initial facts that are true before executing the plan. The output of the planning function is a sound plan that, when executed, can attain the goals.

Planning in Tactical Plan Recognition is quite different from 'normal' planning. A normal planning algorithm only has to come up with one plan, the best plan. In normal planning all the variables are well known and one can often find the most optimal and detailed plan. In the case of plan recognition, one does not know all the variables that the opponent used in planning. This implies often that the plan recognition system only can come up with less detailed plans (lower gramularity). Also, as it does not know the real goals and initials of the opponent, multiple plans are to be generated, in the hope that one of them is the opponent's actual plan being executed.

\subsubsection{Different granularities}

There are two approaches with regard to the granularity of plans. The first is to generate all complete plans when the first observations enter the system. The second is to start off with partial plans, that are refined by information of new observations.

The advantage of supplying complete plans are:

- Constraints on the plans can be examined. For example: if one states the geographic start point of an object's trajectory and, given the current position of the object, one can check whether the endurance of the object suffices to be able to execute the plan of a plan hypothesis.

- All the planning is done before correllation and it is not necessary to inter-leave planning and correlation.

A disadvantage is that the complete set of plan hypotheses may become large in complex cases. However this is not yet the case here and so we choose to provide completely instantiated plans in the plan hypotheses. However, in the case of the partial order plan, we maintain an incomplete sequence of plan
actions.

The suitability of three planning algorithms is now examined. Moreover the specific characteristics of military plans, such as mission abort, are con-
sidered. 


\subsubsection{Planning algorithms}

In this section three solutions to implement the plan liypotheses generation task, are being examined for their applicability: 1) partial order planning and 2) deterministic finite automaton and 3) fixed sequences of actions. Criteria for the algorithms are: completeness of the set of solutions, handling partial achievement of goals, avoidance of duplicate solutions. Now the algorithms will be discussed in detail.

1. Partial Order Planners (POP) : this is the most efficient planner that applies STRIPS operators. These planners have been around for quite some time and are mentioned in Artificial Intelligence textbooks like [76]. An example of the application of STRIPS operators are given in figure 7.2. Two operators are depicted here: Fly(there), stating the flight of an airplane from here to destination there (here and there are variables) and Photo(target) stating that the airplane takes a photo of the target. The POP algorithm has as input these operators and a goal state, e.g. At(Airbase) $\wedge$ Info(MUN1) and an initial state At(Airbase). The algorithm then searches through sequences and instantiations of the operators from the goal state to the initial state. The solution is a plan with the sequence as displayed in figure 7.2.

Though they have been proven to be very flexible and efficient planners, there is a problem in applying thern to plan recognition. Normally the POP performs a search for one sequence of actions, i.e. a plan, from the goal state to the initial state (specified by the background knowledge $\Gamma$ ). During the search there are some choice points and when any solution is found the algorithm can stop it's search.

In our case, the planning algorithm has to do an exhaustive search for all sequences of actions that lead to the goal state. However, the choice points of the POP algorithm do not exclude all solutions from each other and duplicate solutions may occur. This is troublesome for the probability calculations of the plan recognizer, because the sum of the probabilities cannot be normalized. A solution to this problem is to devise a function that removes duplicates after planning, but this is rather cumbersome.

Furthermore plan failure or only partial goal attainment are not directly considered in the standard POP algorithm. Yet partial achievement of 
STRIPS operators:

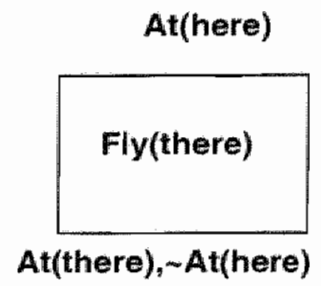

At(target)

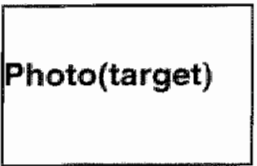

At(target), Info(target)

Plan: Initials: At(Airbase), Goalls: At(Airbase), Info(MUN1)

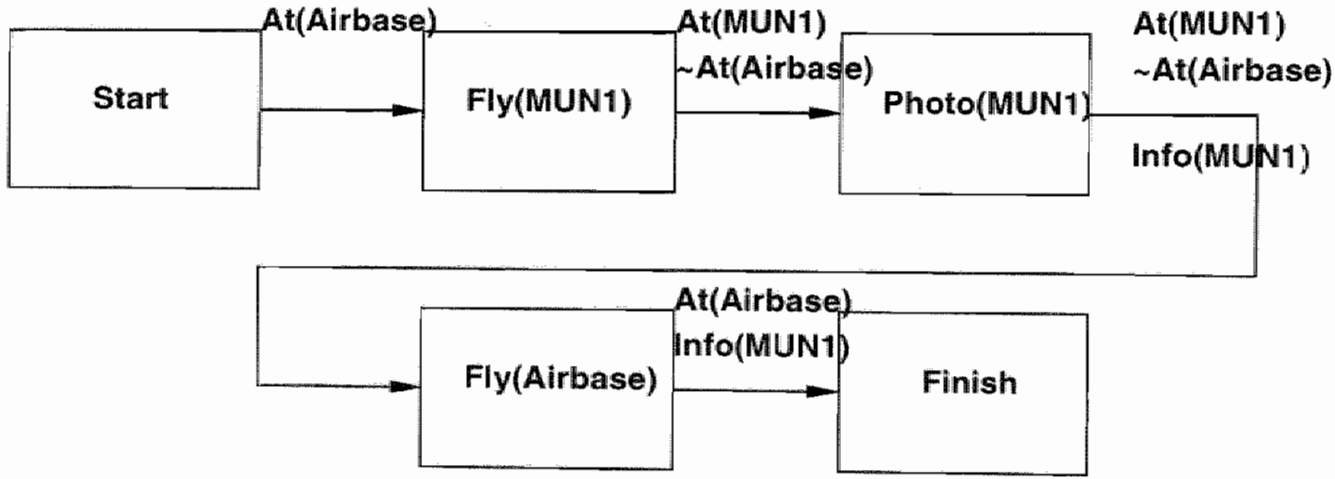

Figure 7.2: An example of STRIPS operators and a plan created by a POP with the STRTPS operators. 
goals occurs often in the military world and indeed in any real world. For example, if the goal is to destroy two targets and only one target can actually be destroyed. Another example is that the goal is to destroy one target, but this cannot be achieved. However, the attacking airplane is able to return safely to the home base. The save return of any friendly airplane to its home base is an always present and high priority goal of military forces. A solution for this problem is to recognize these partial achievement sets of goals and to devise separate plans for them. But this is also rather cumbersome.

Also the complexity of the search for plan hypotheses, as well as the number of plan hypotheses that is generated, can be quite large.

2. Definite Finite Automata (DFA) planners: the DFA specifies legal sequences of actions. It was introduced in the first tactical plan recognizers of Azarewicz $[7,8]$ as a plan template for single-agent plans. It differs from the STRIPS planner that decides on a partial order of the actions during the search for a plan. In the case of the DFA planner, all possible and legal sequences of actions are already entailed in the DFA itself. This planner is very well suited for the procedural way of working of the armed forces. Moreover, plan failure or partial plan attainment is easily incorporated into the DFA. However, only plans of a limited complexity can be generated by the DFA planner. An example is given in figure 7.3, page 132. In this figure, the ingress sequence denotes actions that are to be done when the object is flying toward its target and the egress sequence concerns actions that are to be done when the object is fying to its home base.

3. Fixed sequences: this method is even simpler than a finite automaton. Only one fixed sequence of events is allowed, imposing a linear (i.e. total) order on the actions. We find this planner in many of the examples of this thesis, as it is easily defined by a logical equivalence and a linear order. Remember the civil aviation example: $\forall x F l i g h t(K L 174, x) \Leftrightarrow$ $[F l y(H, A, x), \operatorname{Stop}(A, x), F l y(A, F, x)]$. The flight KL174 entails the sequence fly from Heathrow to Amsterdam, stop in Amsterdam and finally fly from Amsterdam to Frankfurt. These simple planners are very useful in the early stages of plan recognizer system development or in logical descriptions and inferences for formal properties. 


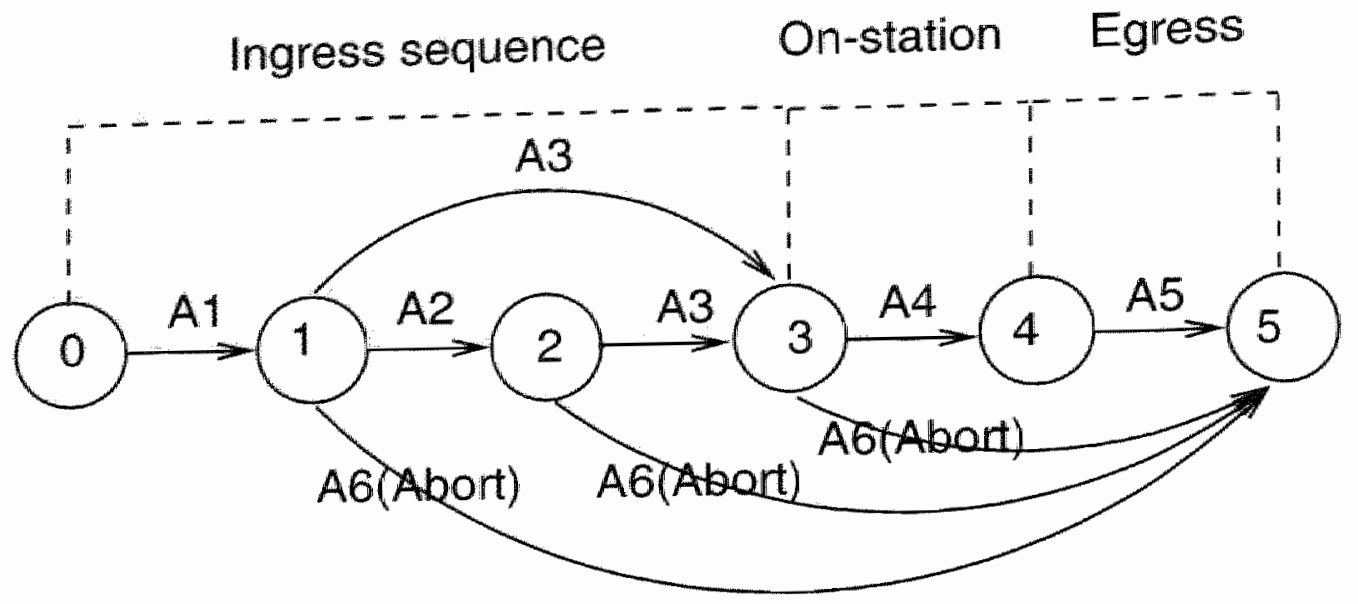

Figure 7.3: An example DFA plan template.

\subsubsection{Soundness and completeness of Planners}

The soundness and completeness of the POP algorithm is described in Russel and Norvig's textbook [76]. In case of the DFA and the fixed sequence templates, these are simply instantiated by means of Universal Instantiation (UI). UI is a sound and complete method.

\subsubsection{The complexity of Planners}

The POP involves significant searching for acceptable sequences of steps. The algorithm is PSPACE-complete [18] and, even for seemingly simple problems, the calculation can take a while. A definition of PSPACE completeness is not readily found in textbooks on discrete mathematics and I must confess that I got the definition from the world wide web. A description of PSPACEcompleteness is quoted from the Wikipedia online encyclopedia [94]:

In complexity theory the class PSPACE is the set of decision problems that can be solved by a Turing machine using a polynomial amount of memory, and unlimited time.

The instantiation of the DFA or fixed sequence template does not a search for possible sequences, but the search of the initials to match the template still has to be done. The complexity is determined by the size of the initial 


\begin{tabular}{|c||c|c|c|c|}
\hline planner & $\begin{array}{c}\text { partial } \\
\text { goals }\end{array}$ & $\begin{array}{c}\text { duplicate } \\
\text { plans }\end{array}$ & complexity & $\begin{array}{c}\text { plan } \\
\text { complexity }\end{array}$ \\
\hline \hline POP & no & yes & PSPACE-complete & high \\
\hline DFA & yes & no & exponential & medium \\
\hline Fixed sequence & no & no & exponential & low \\
\hline
\end{tabular}

Table 7.1: An overview of the characteristics of planning algorithms.

set and the amount of actions in the plan template: $|i t s|^{|s s|}$, where $|i t s|$ is the number of initials and $|s s|$ is the number of actions in the template.

\subsubsection{Evaluation of planners}

In table 7.1 and overview of the characteristics of the three planners is given. The conclusion is that the POP algorithm is not suitable for our Tactical Plan Recognition purposes on account of possible duplicate plans and its inability to handle partial goal achievement. The DFA planner is a good model for the procedural plans one often finds in the military. The Fixed Sequences method is suitable for simple plans.

During plan execution, the observed objects move along legs. For the plan recognizer it is interesting to estimate the parameters of the object's movement; that is to do estimation of the kinematic state. This is the topic of the next chapter.

\subsection{Estimation of the kinematic state in Tactical Plan Recognition}

Observations in the military world always have uncertain measurements of the kinematic state of the observed object. This uncertainty can be modelled by stochastic variables. Given the uncertain measurements, one can estimate the kinematic part of a opponent's planned state. This process of estimation is also called 'filtering'. Here the plan hypotheses have some stochastic variables that represent the kinematic state of the observed object. In tracking, these state variables are used to predict (short term) a new position of the object. In the case of plan recognition, the movement plan concerns longer term motions, 
modelled by a sequence of legs ${ }^{3}$. The planned movement is dictated by the legs, and we only want to estimate the kinematic state of movements along that leg.

The estimation of kinematic stochastic variables of the plan's legs is illustrated by a simple scenario in the first section. Here also the purposes of kinematic estimation are listed. The following sections discuss two stochastic models that can be applied. Finally, an evaluation of the estimation with the two models is given and it is shown that the straight uniform motion gives the best estimation.

\subsubsection{Purposes of kinematic estimation}

In a Tactical Plan Recognition system, one wants to relate the motion of the target along the legs that are a part of the plan and have a fixed heading.

For an example of legs, see figure 7.4 that depicts a plan with two legs, named Leg1 and Leg2. The first leg is the ingress, from air base AB1 to the enemy TK5 unit and the second leg is the egress from unit TK5 to the FARP unit ${ }^{4}$. In the figure, there are two targets: Target 1 flies along the leg and Target 2 crosses the leg. A leg comprises a straight uniform motion between two fixed points. The state vector has to reflect the fixed direction of motion along the leg. ments:

In choosing the kinematic state variables one has to weigh two require-

1. the prediction of the state variables: here we want to relate the prediction closely to the planned leg. The prediction should not be allowed to 'drift' away in position from the leg.

2. The correlation between a planned state and a measurement : the correlation should consider the distance from the measurement to the leg and the heading of the measurement.

For this task a filter has to be designed. This will be covered in the next sections. In the following section, the options for filtering are discussed.

\footnotetext{
${ }^{3}$ A leg describes a linear motion of a target from one begin point to one end point. Often the motion is a linear uniform motion.

\#ngress concerns the flight towards the military front or target and egress concerns the Aight from the front to the home base.
} 


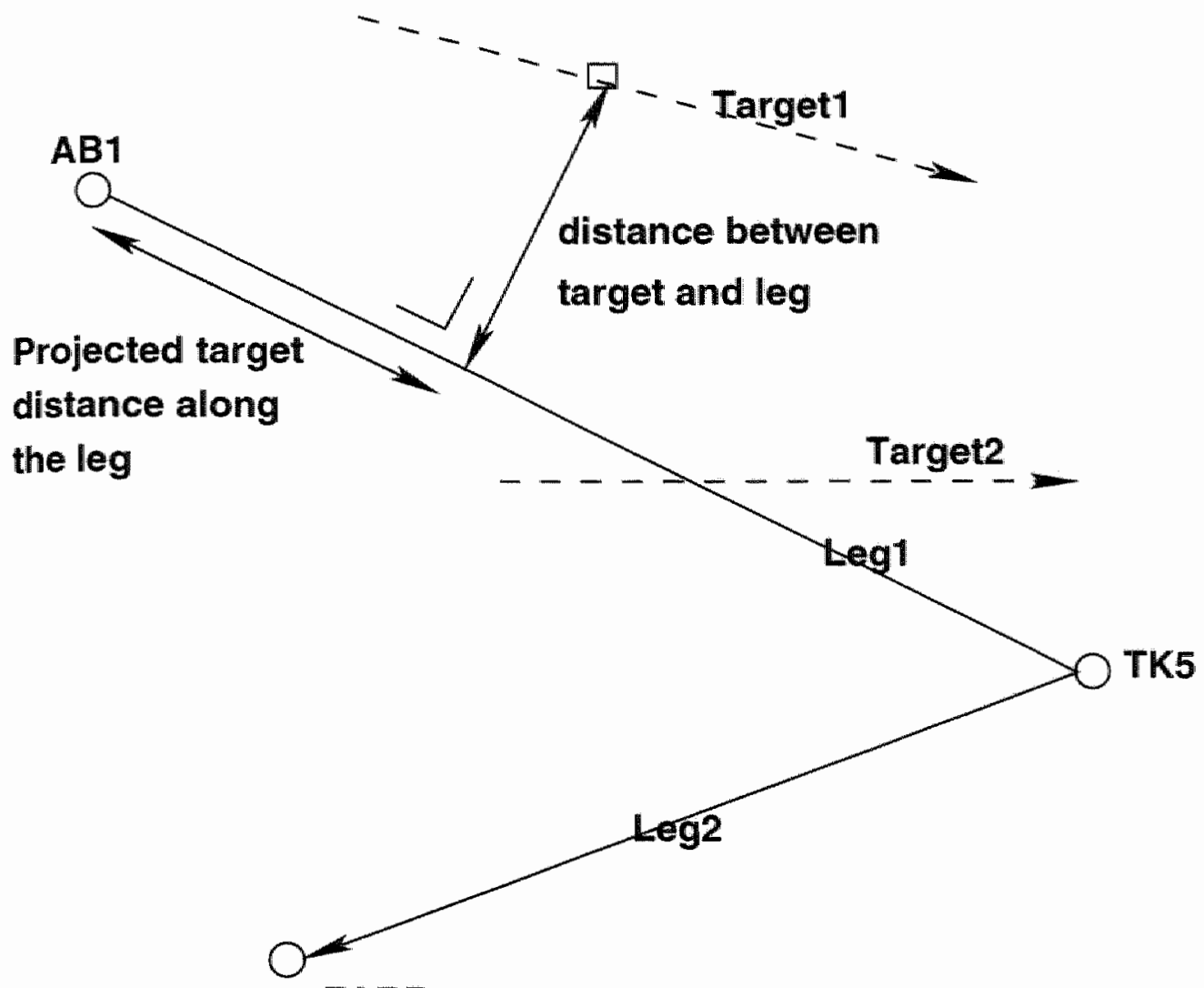

FARP

Figure 7.4: Example of a plan with two legs.

\subsubsection{Options for filtering leg variables}

The position of the target and its direction of flight may differ significantly from the leg's position and direction. So the filter has to entail a projection of the flight's position on the leg. The projection can be accompanied by a filter operation. The following options to estimate the position and speed of an object along a leg are discerned:

- Do not filter: directly project the observation's or position and speed onto the leg.

- Filter and project: first filter the observed positions and then project 


\begin{tabular}{|l||c|c|c|}
\hline & project\&filter & filter\&project & only project \\
\hline \hline observations & OK & OK & OK \\
\hline track updates & NOK & NOK & OK \\
\hline
\end{tabular}

Table 7.2: Filter options for observations and track updates.

the estimated position and speed on the leg.

- Project and filter: first project the measurement or track update and then estimate the position and speed along the leg.

With regard to the input of the filtering, one has to deal track updates and observations. A significant difference between track updates and observations is, that the observations are statistically independent, whereas track updates are dependent. The tracks are dependent because they are the result of combinations of independent measurements in the tracking's filtering. There exist techniques to de-correlate track updates, so the de-correlated track updates can be filtered again. But this is only useful if one wants to fuse the filtering of track upclates with observations or other measurements. Otherwise the track would be simply filtered, de-filtered and filtered again, which is not sensible.

Optimal state estimation algorithms, require their input measurements to be statistically independent ${ }^{5}$. So as input to filtering, independent measurements are preferred. This preference for independent measurements, restricts the options for filtering. An overview of the options is given in table 7.2. Here the 'NOK' elements indicate that the method will work with dependent kinematic vectors, so is 'Not OK'. The 'OK' elements indicate that the kinematic vectors are independent, so the method is 'OK'.

In the numerical example of section 5.3 .3 the input were observations and the 'only project' method was used, so there was no filtering there. In the discussion below the, more advanced, 'project \& filter' method will be applied. This rather than its reverse 'filter \& project" method, because state vectors closely related to the leg system at hand are preferred. Another advantage is that the computational complexity decreases when the number of state variables decreases.

\footnotetext{
"If dependent measurements are entered into the system, the estimation deteriorates and the estimated error is smaller than its actual value.
} 
In the following text, two possible sets of state variables are presented. An analysis of the performance of their estimators is done by an example with the trajectory of target Target2 of figure 7.4.

\subsubsection{Mean reverting process}

A possible set of state variables is given in the mean reverting process model of Mevassvik and Løkka in [52] ${ }^{6}$. Consider the general stochastic differential equation (for a textbook on stochastic processes and filtering see Jazwinski[41]):

$$
d x_{t}=\mu\left(x_{t}, t\right) d t+\sigma\left(x_{t}, t\right) d w_{t}
$$

where $t \geq 0$ and $x_{t}$ is a stochastic variable and $w_{t}$ represents noise. The first term, $\mu\left(x_{t}, t\right) d t$, is the drift term and the second term, $\sigma\left(x_{t}, t\right) d w_{t}$, is called the diffusion term. In a mean reverting process, equation 7.1 becomes:

$$
d x_{t}=\lambda\left(\mu-x_{t}\right) d t+\sigma_{x} x_{t} d w_{t}
$$

This 'mean reversion property' says that the value of the process will always return to $\mu . \mu$ is a centrall location or long term mean of $x_{t}$. The time it takes to revert to the mean depends on the adjustment parameter $\lambda$, the reversion strength.

The model is applicable in our Tactical Plan Recognition case, when one assumes that the speed of a target will not drift away from a long term mean on a leg, but instantaneous diversions of the speed are possible. For this system Mevassvik and Lokka devise the following state vector: $[s, m, v]$. Then use the following discrete system equations:

$$
\begin{aligned}
s(k+1)-s(k) & =T v(k) \\
m(k+1)-m(k) & =0 \\
v(k+1)-v(k) & =\lambda T m(k)-\lambda T v(k)+\sigma_{v} v(k) w
\end{aligned}
$$

where $T$ is the time between two subsequent measurements, e.g. time $k$ and time $k+1, m(k)$ represents the mean velocity, $v(k)$ instantaneous velocity and $s(k)$ represents the distance travelled at time $k$ in the coordinate system. Rewriting of the left side of the equations gives:

\footnotetext{
The mean reverting process is often applied in financial stochastic models.
} 


$$
\begin{aligned}
s(k+1) & =\operatorname{T} v(k)+s(k) \\
m(k+1) & =m(k) \\
v(k+1) & =\lambda T m(k)-\lambda T v(k)+v(k)+\sigma_{v} v(k) w
\end{aligned}
$$

In the Mevassvik's paper, $\lambda=1$. The notation for the system equations for a Linear Time-Invariant (LTI) dynamic system, in this report, are:

$$
\begin{aligned}
s v(k+1) & =\Phi s v(k)+\Gamma w(k) \\
z(k) & =H x(k)+v(k)
\end{aligned}
$$

Where $x(k)$ is the state vector, $\Phi$ is the state transition matrix, $\Gamma$ is the process noise disturbance matrix, $z(k)$ is the measurement vector, $H$ is the measurement matrix, $w(k)$ is the process noise and $v(k)$ is the measurement noise. The state vector is here: $s v(k)=[s(k) m(k) v(k)]^{T}$ and the measurement vector is $z(k)=[x(k) y(k)]^{T}$, where $x(k), y(k)$ are the measured $\mathrm{x}$ and $\mathrm{y}$ position.

The corresponding state transition matrix, $\Phi$, and disturbance matrix, $\Gamma$, and finally the measurement matrix, $H$, follow from the system equations 7.5 :

$$
\begin{gathered}
\Phi=\left[\begin{array}{lll}
1 & 0 & T \\
0 & 1 & 0 \\
0 & \lambda T & (1-\lambda T)
\end{array}\right] \Gamma^{\prime}=\operatorname{Jacobian}\left(\left[\begin{array}{l}
1 \\
1 \\
v
\end{array}\right]\right)=\left[\begin{array}{lll}
0 & 0 & 0 \\
0 & 0 & 0 \\
0 & 0 & 1
\end{array}\right] \\
H=\left[\begin{array}{lll}
\sin h_{l e g} & 0 & 0 \\
\cos h_{l e g} & 0 & 0
\end{array}\right]
\end{gathered}
$$

The system is observable when $\operatorname{rank}\left(H^{T}, \Phi^{T} H^{T}, \Phi^{T} P h i^{T} H^{T}\right)$ equals the number of state variables, i.e. 3 . The matrix is:

$$
\left[\begin{array}{llllll}
s & c & s & c & s & c \\
0 & 0 & 0 & 0 & s \lambda T^{2} & c \lambda T^{2} \\
0 & 0 & s T & c T & (1-\lambda T) s T+s T & c T+c(1-\lambda T) T
\end{array}\right]
$$

where $c$ is $\cos h_{\text {leg }}$ and $s$ is $\sin h_{\text {leg. }}$. If $T \neq 0$ and $\lambda \neq 0$, the rank of the matrix equals three, so the system is fully observable. The optimal estimator for this linear, time-invariant problem, when the noise is Gaussian, is the Kalman filter. 


\subsubsection{Constant velocity along planned leg}

Mulder and Pointeau [63] only use the distance along the planned leg and the speed along the planned leg and discard the mean reversion strength parameter. This gives the following stochastic differential equation:

$$
d x_{t}=x_{t} d t+\sigma_{x} x_{t} d w_{t}
$$

The system equations thus are:

$$
\begin{aligned}
s(k+1) & =s(k)+T v(k)+w \\
v(k+1) & =v(k)+w
\end{aligned}
$$

where: $T$ is again the time between two measurements, $s(k)$ is the distance covered by the target along the trajectory and $v(k)$ is the speed of the target and finally $w$ represents gaussian noise with zero mean and some variance on the state variable.

The state variables are related to the measurement as follows:

$$
x=s \sin h_{\text {leg }}, y=s \cos h_{\text {leg }}
$$

where $x, y$ give a position in cartesian coordinates centered at the start of the planned leg, $h_{\text {leg }}$ denotes the heading of the planned leg. The system matrices in the kinematic system equations can be derived directly from the equations above.

$$
\Phi=\left[\begin{array}{ll}
1 & T \\
0 & 1
\end{array}\right], \Gamma=\left[\begin{array}{ll}
1 & T \\
0 & 1
\end{array}\right], H=\left[\begin{array}{ll}
\sin h_{\text {leg }} & 0 \\
\cos h_{\text {leg }} & 0
\end{array}\right]
$$

Again the Kalman Filter is the optimal estimator for this problem. The second order system is observable when $\operatorname{rank}\left(H^{T}, \Phi^{T} H^{T}\right)=2$. The matrix is:

$$
\left[\begin{array}{llll}
c & s & c & s \\
0 & 0 & c T & s T
\end{array}\right]
$$

where $c$ is $\cos h_{\text {leg }}$ and $s$ is $\sin h_{\text {leg. }}$. If $T \neq 0$, the rank of the matrix equals two, so the system is fully observable. 


\subsubsection{Analysis of the two estimators}

The matrices of the two systems, augmented with the process noise matrix and the measurement noise matrix, are input for the Kalman Filter, that optimally estimates the kinematic state variables for such linear, uncorrelated gaussian systems. As an example the first leg of figure 7.4 and Target2 are used. It concerns a horizontal movement of Target2, that crosses the first leg from point $u 1=[2000.0,7000.0]$ to point $u 2=[8000.0,3000.0]$. The speed of the target is given by $v=100+20 \sin \frac{2 \pi t}{40}$. The observations have a distance accuracy of $400[\mathrm{~m}]$. The reversion strength parameter is set to one: $\lambda=1$. The process noise matrix is complete and has as elements $\sigma_{s}=10[m]$ and $\sigma_{v}=10[\mathrm{~m} / \mathrm{s}]$. The measurement noise matrix is complete and has values: $\sigma_{x}=10[m], \sigma_{y}=10[\mathrm{~m}]$. Now the performance of the filters is tested by means of Monte Carlo simulations. The amount of simulations is given by:

$$
M=\frac{1}{2 \epsilon^{2}} \ln \frac{2}{\delta}
$$

from [16]. Here $M$ is the amount of Monte Carlo simulations to be done, $\epsilon$ is the required accuracy of the estimated mean and $\delta$ is the confidence in the estimated mean. If we choose $\epsilon=10 \%$ and $\delta=99 \%$ we need 16 Monte Carlo simulations.

\begin{tabular}{|c|c|c|}
\hline parameter & mean error & standard deviation error \\
\hline \hline \multicolumn{3}{|c|}{ Mulder and Pointeau } \\
\hline distance $[\mathrm{m}]$ & 0.0363 & 8.1625 \\
\hline speed $[\mathrm{m} / \mathrm{s}]$ & 0.0070 & 3.3743 \\
\hline \multicolumn{3}{|c|}{ Mevassvik and Løkka. } \\
\hline distance $[\mathrm{m}]$ & -0.0500 & 8.0874 \\
\hline speed $[\mathrm{m} / \mathrm{s}]$ & 26.7705 & 39.1329 \\
\hline
\end{tabular}

These figures show that the mean reverting process is less accurate than Mulder and Pointeau's model in estimating the speed of the target. The speed estimation error is significantly larger for the mean reverting process. In the scenario the target's leg and the planned leg have an angle of about 30 degrees. To examine the sensitivity of the filters with regard to this angle, the Monte Carlo simulations were repeated for angles in the range of -30 till 20 degrees. The ranges here are given in the table below: 


\begin{tabular}{|c|c|c|}
\hline parameter & mean & standard deviation \\
\hline \hline \multicolumn{3}{|c|}{ Mulder and Pointeau } \\
\hline distance & {$[-0.5,+0.5]$} & {$[8.0,10]$} \\
\hline speed & {$[0,-0.3]$} & {$[3.4,4.8]$} \\
\hline \multicolumn{3}{|c|}{ Mevassvik and L $6 \mathrm{kka}$} \\
\hline distance & {$[-0.5,+0.5]$} & {$[8.0,11]$} \\
\hline speed & {$[26,31]$} & {$[62,50]$} \\
\hline
\end{tabular}

With a varying interception angle, the performance of the filters does not vary significantly. The order of the error remains the same. 


\section{Chapter 8}

\section{An algorithm for identified observations}

In this chapter, the task of the Generic Task Model for identified observations, 6.1, page 112, are implemented by a generate and test algorithm that generates and maintains a consistent and complete set of plan hypotheses. The hypotheses are based on previous observations and when a new observation is entered into the system, it retains only those hypotheses that are consistent with the new observation.

The algorithms are implemented in the Haskell programming language that will be described in section 8.1. Subsequently the algorithm is presented and its formal properties are examined. It it will be shown, that the algorithm only works for a restricted class of plan hypotheses.

\subsection{The Haskell programming language}

In the functional programming language Haskell, mathematical constructs of data: sequences and tuples, are directly used with pure, strict or non-strict functions. Implementations of algorithms in Haskell can be extremely efficient in the sense of the amount of code required. However, it is efficient only for suitable algorithms, i.e. algorithms that are inherently recursive. Introductions to the Haskell language can be found in [14,88].

Haskell's main data structures are lists and tuples. In the algorithms sets are represented by lists $[x 1, x 2, \cdots, x n]$. Set operations such as element of, union and intersection are applied to the lists. Through a list comprehension: 
$[x \mid x-x s, x<1.0]$ new lists are constructed. The list can also be used for multisets (also called bags) and sequences and their operations, but also for normal sets and finally for sequences. However, their operations will be implemented differently. The concatenation of lists is done by the ++ operator.

A pure functional language consists solely of function calls without any side effects. In Haskell, functions with uncurried parameters are applied. Here function parameters are not specified as a tuple but just separated by spaces: i.e. $f(x, y)$ is written as $f x y$. The functions and variables both start with a lower case letter. The cases of piecewise functions are represented as follows:

- comment is preceded by two dashes -.

- by a number of condition $=$ statements. The otherwise $=$ condition is always true.

$$
\begin{array}{ll}
\text { abs } x & \mid x>0=x \\
& \mid \text { otherwise }=-x
\end{array}
$$

- by specifying a number of patterns for the variables

-afactorialfunction

$$
\begin{aligned}
& \text { fac } 0=1 \\
& \text { fac } n=n * f a c(n-1)
\end{aligned}
$$

Wildcards ' - ' can be used in the patterns. In the following example, the first parameter is of no interest: $f-s=1.0$.

Composition of functions is indicated by a bullet: $f(g x) \equiv f \bullet g x$. Variables used in a function are given after the "where" statement, and are lazy evaluated.

$$
\begin{aligned}
\sin 2 x= & \sin y \\
& \text { where } \quad y=2 x
\end{aligned}
$$

For lists (e:es) denotes the first element $e$ of a list and its other elements are es." [" denotes an empty list or empty set $\emptyset$.

The function partition $p s$ divides the set, sequence or list $s$ into two partitions, according to the function $p$. The example below partitions the set of integer numbers by a lambda function $(\lambda \cdots)$. The output of the partition function is a tuple of two sets, the first set consists of those elements of $s$ that 
satisfy the lambda function, the second set consists of elements that do not satisfy the lambda function:

$$
\left(\mathcal{Z}^{-}, Z^{+}\right)=\text {partition }(\lambda x \rightarrow x<0) \mathcal{Z}
$$

where: $\lambda x \rightarrow x<0$ is a lambda function. There are numerous other standard Haskell functions, mostly operating on lists, but these will be elucidated in the text when they occur.

Finally, the Haskell language has 'lazy evaluation'. An expression is only evaluated when it is actually required. This allows any sequence of expressions (such as functions and data structure definitions) in the program code. The lazy evaluation builds a dependence graph of these expressions and the graph is traversed by the Haskell interpreter.

The Haskell code that is used in the main text still is a somewhat simplified version of the "real' programs.

\subsection{Algorithm}

First, the generate and test algorithm is given below. The main function is plan_recognizer. The generate_hypotheses ${ }^{1}$ function is the generate part of the algorithm and the hypotheses_management is the test part of the algorithm. The functions hypotheses_assessment and hypotheses_selection limit the number of the plan hypotheses.

Algorithm 8.1 Plan recognition algorithm for single plan / identified observations.

1: - the termination function pattern

2: plan_recognizer .... - [J = I]

3: - main function pattern

4: plan_recognizer $k$ its oprs ps $(0: 0 s)=$

4: kps: plan_recogniser $k$ its aprs ps1 os

5: where

6: $\quad p s 1=$ if $p s==\|$

7: $\quad$ then generate_hypotheses its oprs o

8: $\quad$ else hypotheses_management o ps

9: $\quad$ ps2 $=$ hypotheses_assessment o psI

\footnotetext{
The hypotheses are plan hypotheses here, as it concerns identified observations.
} 
10 :

$$
k p s=\text { hypotheses_selection } k \text { psi }
$$

11:

12: hypotheses_management $p s=$

13: $\quad$ I update-hypothesis $p o \mid p \leftarrow p s, p$ consistent with of

14:

15: generate_hypotheses $=$ planner oprs $\bullet$ problem_definition its o 16:

17: hypotheses_assessment ps o $=\int(p$, corr_prob po $\left.o) \mid p \leftarrow p s\right]$

18:

19: hypotheses_selection $k p s=$ take $k \cdot$ sort $p s$

The symbols used in the algorithm are: os equals $\mathcal{O}$ a set of observations and $o$ is one observation, $p s$ is the set of plan hypotheses, i.e. the set of current plan hypotheses, $p$ is one plan hypothesis (in the formal definitions $p$ was called $\phi), P(p)$ is a function that returns the ranking of a hypothesis ${ }^{2}$. Note that $p s 1, p s 2$ are intermediate variables. Furthermore, its are the initial facts and oprs are the plan templates. Examples of plan templates are the Flight $(K L 175, x) \Leftrightarrow \cdots$ and Flight $(K L 175, x) \Leftrightarrow \cdots$ logical equivalences of example 5.1 .

The workings of the algorithm will be explained now. The function is called with the ps parameter being an empty set: plan_recognizer 10 its oprs [] os. This matches with the function pattern at line 4. The lazy evaluation requires that output $k p s$ is generated. To do this, first $p s 1$ is generated by generate hypotheses of line 7 . It is a complete set of plan hypotheses that can explain the first observation. The hypothesis generation function is defined at line $\$ 5$. Here first the goals that the observed target might have, are generated. The goals are input to the planning function.

The likelihood of each plan hypothesis is calculated in the hypothesis assessment function of line 9 and line 17 and its result is available in the variable ps2. Then the l-best hypotheses are selected by the hypothesis_selection. function of line 10. The k-best hypotheses are stored in the $k p s$ variable and constitute the first element of the output list of hypothesis sets. The rest of the output list is generated by a recursive call to the plan_recognizer function at line 4 .

\footnotetext{
${ }^{2}$ By example a probability or a likelihood of the hypothesis $p$.
} 
However, now the ps parameter consists of this first set of plan hypotheses. So the check at line 6 fails and the function hypotheses_management is called. Now, no new hypotheses are created, but the current set of hypothesis is filtered by the consistent_with predicate. So in the $p s 1$ variable at line 6 , one will find only plan hypotheses are are consistent with the second observation.

This sequence of recursive calls is continued, until the observations parameter $o s$ is empty. Then the call matches with the first pattern, at line 2 and the function terminates.

\subsubsection{Consistency check}

The hypothesis management takes care that the set of plan hypotheses is consistent with the observations. Let $\Phi$ be a set of plan hypotheses, then a consistent subset of it is given by:

$$
\{p \mid p \in \Phi, \Gamma \cup \circ \not \neq \neg p\}
$$

In complex logical systems, is not easy to automate the inconsistency reasoning. In our case of simple plan templates, the plan hypotheses are directly related to the observations. They are related by single logical equivalence and simple constraints, e.g. temporal constraints, geographic constraints and con straints on fuel consumption. These constraints concern ' $>$ ', ' $\leq$, relations and their counterparts. These relations are closed: e.g. $\neg(a>1.0) \equiv a \leq 1.0$, and here the consistency can be checked by some predicate. So let us define an infix predicate 'consistent with "and then the set is described as:

$$
\{p \mid p \in \Phi, p \text { consistent with o\} }
$$

In the consistent predicate, constraints that exist between the observation and a plan hypothesis are checked. If the constraints are not satisfied, the plan hypothesis is considered inconsistent with the observations and the predicate returns false. Though not applicable to this algorithm, the consistency check can be expanded to a set of observations. In definition 5.1, page 80, the actual requirement was: $\forall o \in \mathcal{O}, p$ consistent with $o$.

\subsubsection{Propositions}

To expand the above expression to an arbitrary size set of observations, we can specify a recursive function $\mathrm{hm}$ (that resembles the hypothesis_management 
function) for the consistency checks. This is defined in algorithm 8.2. This algorithm is actually the basis of the complete algorithm 8.1.

\section{Algorithm 8.2 Retaining consistency:}

$h m p s[]=p s$

$h m p s(0.0 s)=h m p s^{\prime}$ os

where $p s^{*}=[p \mid p \leftarrow p s, p$ consistent with of

The function is sound, when the complete output set of plan hypotheses is consistent and when the explanation properties of the plan hypotheses is retained by the function.

Concerning the consistency, the following proposition is true:

Proposition 8.1 Retaining consistency: the function 'hm ps os' of algorithm 8.2 generates a set of plan hypotheses, that is consistent with all observations of the set observations, that is: $h m p s$ os $=[p \mid p-p s, p$ consistent with os $]$

Proof Retaining consistency: the proof of a proposition about a recursive function with finite lists is done by structural induction in the realm of purely functional languages. If we malie some assumptions about the consistent_with predicate (abbreviated to $c w): c w \| l=$ true and $p c w(o s 1 \cup$ os 2$)=p$ cw os 1 $\wedge p c w$ os 2 and also the assumption that the result of the application of a reduced set of plan hypotheses, $p s^{\prime} \subset p s$ to $\mathrm{hm}$ results in the following intersection: $h m p s^{\prime} o s=p s^{\prime} \cap h m p s$ os, then we can deduce the following proof:

$$
\begin{aligned}
& \text { base case } \quad: h m p s[l=|p| p+p s, p \text { cw }[] \\
& \text { left hand side : hmps } \|=p s \\
& \text { right hand side }:|p| p \leftarrow p s, \text { true } \mid=p s \\
& \text { induction step : hmps (o:os) }=|p| p-p s, p \text { cw }(0: 0 s)] \\
& \text { left hand side } \quad \text { hmps (o:os) }=h \mathrm{~m}[\mathrm{ps} \mid \mathrm{p} \leftarrow \mathrm{ps}, \mathrm{p} \text { cw [ol] os } \\
& \text { : }[p s \mid p \leftarrow p s, p \text { cw }[0]] \cap h m p s \text { os } \\
& \text { right hand side : }[p \mid p \leftarrow p s, p c w(o: o s)] \\
& :[p \mid p \leftarrow p s, p \text { cw }[o] \wedge p \text { cw os] } \\
& :[p \mid p \leftarrow p s, p c w[o /] \cap[p \mid p \leftarrow p s, p c w o s] \\
& :[p s \mid p \leftarrow p s, p c w[o]] \cap h m p s \text { os }
\end{aligned}
$$


For both the base case and the induction step, the results of the left hand side and the right hand sides are equal, so the proposition 8.1 holds.

Now look at the retainment of the explanation property.

Proposition 8.2 Retaining properties: if all the hypotheses of the parameter $p s$ have property ' $p r$ ", so ' $p r$ ' is true, then also all the hypotheses of ' $h m p s$ os' have property ' $p r$ '.

Proof Retaining properties: the property 8.1 states: $h m$ ps os $=|p| p+$ $p s, p c w$ os $/$. We would like to add an initial property $p r p$ to each plan hypothesis: $\forall p \leftarrow p s$, pr $p$. That the property is retained by the algorithm is shown as follows: $h m[p \mid p \leftarrow p s, p r p]$ os $=[p \mid p \leftarrow p s, p r p] \cap h m p s$ os $=$ $[p \mid p \leftarrow p s, p r p] \cap[p \mid p \leftarrow p s, p$ cw os $]=[p \mid p \leftarrow p s, p$ cw os $\wedge$ pr $p] \cdot \square$

In the case that $p r$ is the explanation property, this will still be true after the $h m$ operation.

\subsubsection{Soundness}

An output hypothesis a plan recognition algorithm for identified observations is sound given the input set of observations, if the output satisfies the requirements of definition 5.6. So the plan hypotheses are expected to be consistent with the observations and the background knowledge and the plan hypotheses can explain the observations.

For the soundness of the tactical plan recognizer, we look at such properties for its subtasks. In figure 8.1 the cooperation between the subtasks is displayed. If we follow the processing as depicted in the figure, we start with the generation of 'planning problems' based on the first observation by the problem_definition function. In section 7.2 it was already ascertained that the problem definition task was sound and complete by identifying these properties for the forward chaining inference mechanism.

The sound and complete set of goals and initials are input for the planning function. This is tasked with finding all possible plans for a set of goals and initials. In section 7.3 some sound and complete planning methods have been discussed.

The combination of the problem definition and planning tasks corresponds with the generate hypotheses its oprs ofunction in algorithm 8.1. 

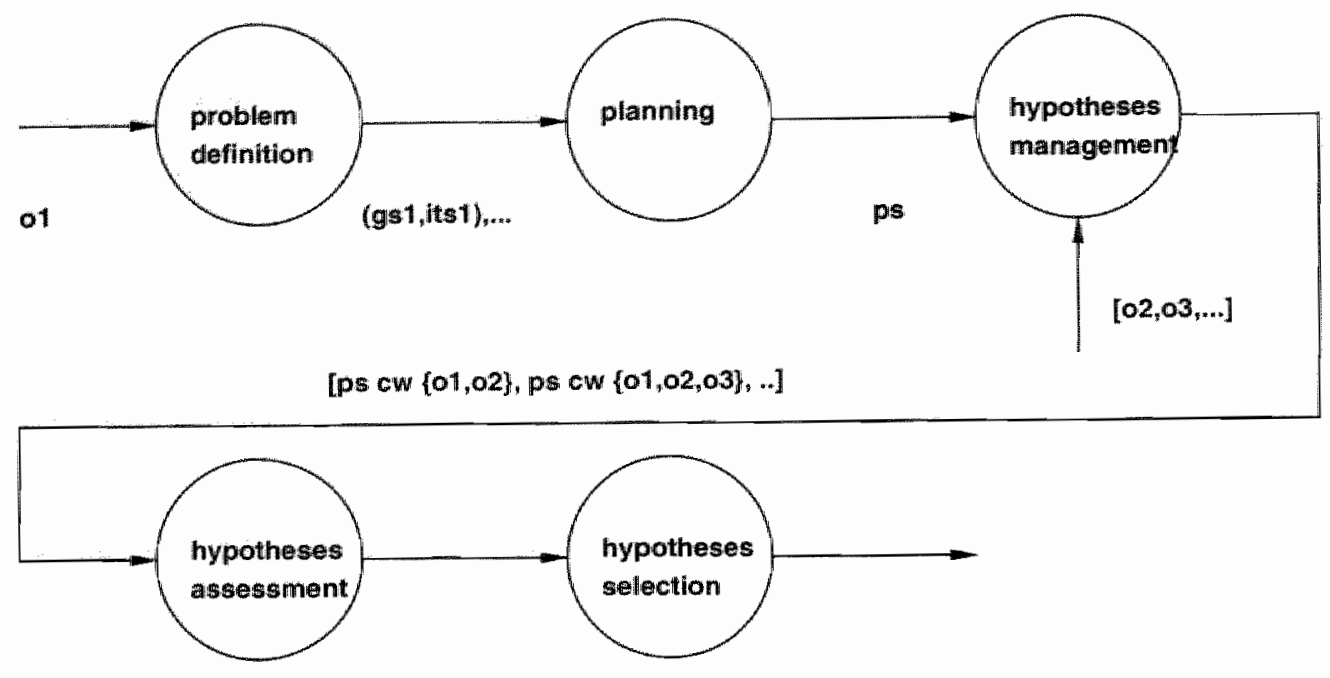

$[\{(p 1, g x y 12),(p 2, g x y 13), \ldots\}, \ldots]$

Figure 8.1: The input and output of the tasks of the Tactical Plan Recognizer.

Now let us consider whether the generated plans are sound and consistent with regard to the first observation ol.

1. The consistency requirement: $\Gamma \cup\{o 1\} \nvdash \neg p \equiv p c w$ ol. As the algorithms of the functions problemdefinition and planning are sound themselves, the resulting plans are true solutions. The planning algorithms will not generate negative actions, so they will never be inconsistent with the first observed action. Even one of the actions will refer to the observation of

2. The explanation property: $\Gamma \cup\{p\} \vDash o \mathbb{l} \equiv p r p$. If assumption 5.1, page 105, holds for the generated plans, the plan, because one of the plan's actions will refer to the observation ol. For the plan to be true, the referring action has to be true.

Now we have established the consistency and explanation property of the set of plan hypotheses with regard to the first observation. The next step in the algorithm is done by the hypothesis_management task, that removes plans that are not consistent with the following observations $02,03, \cdots$. 
When new observations are entered into the plan recognizer, the initial set of plan hypothesis is reduced to a set that is also consistent with the new observations, according to proposition 8.1. So this reduced set is still consistent for all observations entered into the system. Also, according to proposition 8.2 , the explanation property for the original observation of the plan hypothesis is retained in when it is processed.

For the formal properties the hypothesis_management functions and the generate_hypotheses functions are of interest. The hypotheses_assessment function only adds a number to a plan hypothesis and the hypothesis_selection function only restricts the number of hypotheses. Both functions have no influence on the content of the plan hypotheses, but are required for a practical algorithm.

\subsubsection{Completeness}

An algorithm was complete when it satisfies the statement of definition 5.7. It maybe remarkable for the reader, but the algorithm is certainly not complete if we only consider the output of the algorithm.

First, because the hypothesis selection removes valid plan hypotheses before outputting them. However, if this function is removed, the completeness is determined by the generate.hypotheses function. Of course, this function is a composition of the problem definition and planning algorithm and is complete, because its individual functions are.

Second, the algorithm only considers the first observation to generate a complete set of plan hypotheses. As the plans are only based on the first observation, they cannot be complete for all observations. Not all possible plan hypotheses, that can explain all observations, are created by the algorithm, but only all those hypotheses that can explain the first observation. But when one considers the CWAs 4.1 and 4.2 , it can be assumed that the initial set of plan hypotheses, based on the first observation, was already complete and that no new plan hypotheses can be generated by incorporating information of successive observations.

\subsubsection{Complexity}

The memory complexity of the algorithm is determined by the initial set of plan hypotheses for the first observation. During the processing of the next 
observations, the number of plan hypotheses can remain the same or even reduces, when inconsistent plan hypotheses are detected.

The computational complexity of the algorithm is also determined by the number of plan hypotheses, indicated by $N_{p p}$. In the table below an overview of the processing requirements, for processing an observation, is given:

\begin{tabular}{|c|c|}
\hline function & computational complexity \\
\hline \hline generate_hypotheses & $C_{g e n e r a t e}$ \\
\hline hypotheses_management & $N_{p}$ \\
\hline sort & $N_{p} \log N_{p}$ \\
\hline hypotheses_assessment & $N_{p}$ \\
\hline hypotheses_selection & $k$ \\
\hline \hline sum & $f\left(N_{p}\right)=C_{\text {generate }}+2 N_{p}+N_{p} \log N_{p}+k$ \\
\hline
\end{tabular}

Where $C_{\text {generate }}$ is a constant (with regard to $N_{p}$ ) and $k$ is the number of best output hypotheses selected. The sum total of operations is given by $f\left(N_{p}\right)$. In the 'order' notation, the computationall complexity is polynomial with the size of the hypothesis set: $\mathcal{O}\left(f\left(N_{p}\right)\right)=\mathcal{O}\left(C+2 N_{p}+N_{p} \log _{2} N_{p}+k\right)=$ $\mathcal{O}\left(N_{p}^{2}\right)$

\subsubsection{Hypotheses assessment}

This function ranks the plan hypotheses, for example by a probability. Here several methods for modelling uncertainty can be applied. Bayesian nets were first applied by Charniak and Goldman, [25], applying their earlier research in such nets. They where followed by Pynadith and Wellman, [71] and several other authors. The Dempster-Shafer theory for reasoning about uncertainty, was applied in plan recognition in the area of natural language understanding by Carberry, [22], and by Baner, [10] in the area of intelligent user interfaces. For our purposes the assessment of an individual plan hypothesis is not important.

In this research, hypotheses assessment of plan hypotheses is not examined. In this report, the focus is on the more general case of plan recognition by unidentified observations. Here the assessment of assignment hypotheses is done by applying Reid's formula (equation 5.5). 


\section{Chapter 9}

\section{An algorithm for unidentified observations}

In the previous part, an algorithm for the recognition of tactical plans with identified observations was discussed. This algorithm is the basis of a more complex algorithms for Tactical Plan Recognition with unidentified observations. Also the discussion about formal properties of the simpler algorithm are adapted to the unidentified observations algorithm.

We start with a discussion of the data structures of the algorithm, followed by a description of the actual algorithm. In the last part of the chapter, the formal properties of the new algorithm are discussed.

\subsection{Data structures}

For the algorithm development introduce the result of the correlate phase, at correlation.

Definition 9.1 A correlation is a surjective relation between the hypothetical plans and observations: $\mathcal{O} \times \Phi \cdot \square$

So each correlation is a pair $(o, p)$, where $p$ is a bypothetical plan and $o$ is an observation. The correlations divide the set of plan hypotheses also in a number of subsets. In practice the information is translated to the tuple (updote $p o, p, \mathcal{N}(y, B)$ ). The elements of the tuple are: update $p o$ : the correlated plan updated with the new observation (this is a CP plan hypothesis!), $p$ : the 
original parent plan hypothesis (can be of type CP or NP) and the innovation of the correlation: $\mathcal{N}(y, B)$.

The plan hypotheses and assignment hypotheses are organized in clusters. A cluster is a plan recognition problem that is independent of the plan recognition problems of other clusters.

Definition 9.2 A cluster is a tuple $\left(\Phi, \Psi_{h}\right)$, where $\Phi$ is a set of plan hypotheses, called the plan hypothesis list, and $\Psi_{k}$ is a set of assignment hypotheses. $\square$

At some point of time there may be several clusters in a plan recognition system. The performance of a plan recognizer can be boosted if it maintains a set of clusters, because the clusters implement data partitioning. However the plan recognizer can equally maintain only one cluster, containing all the information and derive equal results as the clustered plan recognizer. But here the complexity will be much larger.

An assignment hypothesis consists of a set of references to elements of the plan hypothesis list and an assignment probability.

Definition 9.3 An assignment hypothesis, $h \in \Psi_{h}$ is a tuple: $(p s, P(h))$, where $p s$ is a set of plan hypotheses and $P(h)$ is the probability of the assignment hypothesis. ps is a subset of the plan hypothesis list of the cluster of the assignment hypothesis and is called the plan hypothesis list of the assignment. hypothesis. $\square$

Now some characteristics of the data involved in the plan recognition for unidentified observations are given. They are identical to those tracking. The first property is that the union of the plan hypothesis lists of all assignment hypotheses of a cluster, gives the plan hypothesis list.

Proposition 9.1 Uhehs(c) $p s_{h}(h)=p s_{c}(c)$, where $c$ is a cluster, $h s(c)$ is a function that gives the set of assignment hypotheses of the cluster and $p s_{c}(c)$ is a function that gives the plan hypothesis list of the cluster and $p s_{h}(h)$ is a function that gives the plan hypothesis list of an assignment hypothesis.

The plan hypotheses themselves are quite unique in the case of a probabilistic plan recognizer. Each plan hypothesis represents an unique correlation option between a parent plan hypothesis and a plot. However, one observation can update many plan hypotheses, so the combination of root observation and updating observation does not give a unique index for a plan hypothesis. This property was already summarized in proposition 5.5 . 


\subsection{Algorithm}

The algorithm handles observation by observation; they are handled one at a time in an ordered sequence of increasing time. The assignment hypotheses are devised for a set of observations of multiple observers, such that all observers can be assumed to have scanned over the objects of the plan hypotheses of the assignment hypotheses. The algorithm does not account for the clustering, as this greatly complicates the processing of the algorithm.

Algorithm 9.1 plan recognition algorithm for non hierarchical plan / unidentified observations.

1: plan_recognizer $\ldots .-[]=[]$

2: plan_recognizer $k$ its oprs $(p s, h s)(o: o s)=$

3: $\quad[k h s]++$ plan_recogniser $k$ its oprs $\left(p s^{\prime \prime}, h s^{\prime}\right)$ os

4: where

5: $\quad$ - decide upon best assignment hypotheses

6: $\quad(p s a, h s a)=$ if scanned hs then reduce $l(p s, h s)$ else $(p s, h s)$

$7:$

8: $\quad$ - correlate the new observation with the reduced assignment

9: $\quad$ corrs $=$ correlate o psa

10: $\quad\left(p^{\prime}\right.$, hs' $\left.^{\prime}\right)=$ if corrs $==$ /] then

11:

12:

create its oprs o

13:

else

update its oprs corrs (psa, hsa) o

In the algorithm the following symbols are used: [] equals an empty set $\emptyset, h$ is an assignment hypothesis, $h s$ equals $\left\{h_{1}, h_{2}, \cdots, h_{n}\right\}$ and is a set of: assignment hypotheses. In the terms of the formal definitions about tactical plan recognition, $h$ equalls $\Psi_{h}$. $c$ is a correlation. Corrs stands for the set of correlations and is a binary relation between observations and plan hypotheses: corrs : $\mathcal{O} \times \Phi$. An element is a tuple $(o, p)$ (see definition 9.1), where $p$ is a hypothetical plan and $o$ is an observation. A correlation states a possible assignment between an observation and a plan hypothesis. $\Gamma$ equals the tuple (its, oprs) and is the background knowledge consisting of the set initials and of the set of operators. 
Now the workings of the algorithm will be tracked. The actual call is like plan_recognizer 10 its ops $(d, / D)$ os. We start with the first observation entered into the plan recognizer. The set of assignment hypotheses, $h s$, from the previous scans is empty, so the function scanned on line 6 returns false and no reduction of the assignment hypotheses is done by the function reduce. In line 3 , ohs represents the out-putted plan hypotheses. It is generated by line 7 and is empty. The Haskell language has lazy evaluation, so the correlate function, line 9, is not called for the first observation. On line 10 of the algorithm, the if statement checks whether the set of hypotheses is empty, and then calls the function create. The create function creates the NP and FA assignment hypotheses for the observation.

So after the first observation is processed the cluster of plan hypotheses and assignment hypotheses has the following elements (that are solely due to the create function):

$$
\begin{array}{ll}
\text { plan hypotheses: } & \mathrm{ps}=\left\{n p 1_{1}, n p 1_{2}, \cdots, n p 1_{n}\right\} \\
\text { assignment hypotheses : } & \mathrm{hs}=\left\{\left\{\phi_{1} \leftrightarrow F A\right\},\left\{o_{1} \leftrightarrow n p 1_{1}\right\}\right. \\
& \left.\left\{o_{1} \leftrightarrow n p 1_{2}\right\}, \cdots,\left\{o_{1} \leftrightarrow n p 1_{n}\right\}\right\}
\end{array}
$$

Here npv denotes the $w^{\text {th }}$ new plan hypothesis, created to explain observation $o_{v}$ in this case $o_{1}$. Note that this data is internal and no data has been outputted yet. The assigmment hypotheses all are singletons.

When the second observation is entered into the system, the hs set of assignment hypotheses is not empty anymore. The function scanned on line 6 checks whether the sensors have scanned over the set of plan hypotheses and assignment hypotheses. If so, the function reduce decides on the k-best actual assignments. At line 8 the outputted plan hypotheses are generated. If no reduce has been done, no output plan hypotheses are outputted. The function conrelate on line 9 correlates the second observation to psa, the current set of plan hypotheses. Note that psa differs from the output set khs:psa $\neq k$ hs. If there are correlations, the update function, at line 13, uses the correlations to update the current set of assignment hypotheses. If there are no correlations, only the np and FA assignment hypotheses are added to the set of hypotheses.

So after the second observation is processed the cluster of plan hypotheses and assignment hypotheses has the following elements (that are now due to the create as well as the update function): 


$$
\begin{aligned}
\text { plan hypotheses: }= & \left\{n p 1_{1}, n p 1_{2}, \cdots, n p 1_{n}\right. \\
& c p 12_{1}, c p 12_{2}, \cdots, c p 12_{n}, \\
& \left.n p 2_{1}, n p 2_{2}, \cdots, n p 2_{n}\right\} \\
\text { assignment hypotheses: hs }= & \left\{\left\{o_{1} \leftrightarrow F A, o_{2} \leftrightarrow F A\right\},\right. \\
& \cdots \\
& \left\{o_{1} \leftrightarrow n p 1_{1}, o_{2} \leftrightarrow n p 2_{1}\right\}, \\
& \left\{o_{1} \leftrightarrow n p 1_{1}, o_{2} \leftrightarrow n p 2_{2}\right\}, \\
& \cdots \\
& \left.\left\{o_{1} \leftrightarrow n p 1_{n}, o_{2} \leftrightarrow c p 12_{m}\right\}\right\}
\end{aligned}
$$

Here $n p v_{w}$ denotes the $w^{\text {th }}$ new plan hypothesis, created to explain observation $o_{v}$ and $c p x v_{w}$ denotes an updated plan hypothesis with as parent plan hypothesis $n p x_{w}$ and as its updating observation $o_{v}$. The description above concerns internal data, however now the best assigmment hypotheses are outputted. Also the number of correlated plan hypotheses $c p 12_{1}, \cdots, c p 12_{m}$ is less or equal to the number of plan hypotheses that were generated for the first observation, that is $m \leq n$, so only a part of the original plan hypotheses will correlate with new observations and give rise to correlated plan hypotheses.

The next observations are handled similarly to the second observation. But for these observations, also the correlated plan hypotheses of the previous observations are open to correlation with a new observation and will give rise to new correlated plan hypotheses. To limit the number of hypotheses pruning is done by the reduce function. When reduction is to be done, is determined by the predicate scanned $h s$, that detects when no new observations of previous observers are to be expected.

\subsubsection{Functions}

Now the individual functions, create, update and reduce of algorithm 9.1 will be elucidated. We start with the create function of algorithm 9.2 . 
Algorithm 9.2 create function.

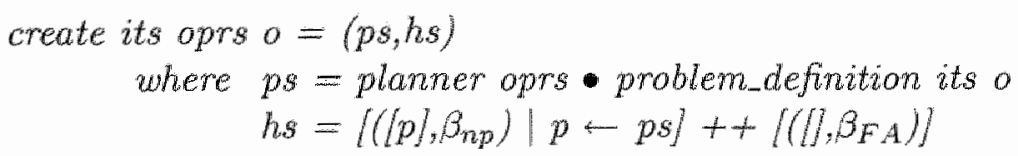

The individual functions: problem_definition and planner are described in sections 7.2 and 7.3. First the new observation is related to possible goals by the problem_definition function. Each goal of the set that is generated by problem_definition can be achieved by a set of plans, generated by the planner function. This function takes as input the background knowledge and a goal, and returns all possible plans, that can achieve the goal. Then, for each goal, all possible plans that can achieve the goal are created by the planner function.

The correlate function, algorithm 9.3, is a simple list comprehension. Here predict $p$ (time o) predicts the plan hypothesis p's state to the time of the observation $o$, the function update' $p$ o updates a plan hypothesis $p$ with an observation, $\mathcal{N}(y, B)$ gives a normal distribution's value for an innovation $(y, B)$ and innovation $p o$ calculates the innovation between an plan's predicted state and an observation:

Algorithm 9.3 correlate function.

$\begin{aligned} \text { correlate }\left(p s,,_{-}\right) a= & ((\text { update' } p o, p, \mathcal{N}(\text { innovation } p o)) \mid \\ & p-p s, \\ & p^{y}:=\text { predict } p \text { (time o), } p^{3} \text { consistent with of }\end{aligned}$

The correlations and the current hypothesis set are input of the update function, that is given below.

Algorithm 9.4 update function.

extend $N P \operatorname{np}(p s, p r)$ oprs its $=\left(n p: p s, p r * \beta_{N P}\right)$

extend $F A(p s, p r)=\left(p s, p r{ }^{*} \beta_{F A}\right)$

extend $C P\left(u p, o p, g_{x y}\right)(p s, p r)=\left(u p:(p s-o p), P_{D} * g_{x y}{ }^{*} p r /\left(1-P_{D}\right)\right)$

update its oprs corrs (ps,hs) $0=(n p s++c p s++p s, n h s)$

where nps = planner oprs $\bullet$ problem_definition its a 


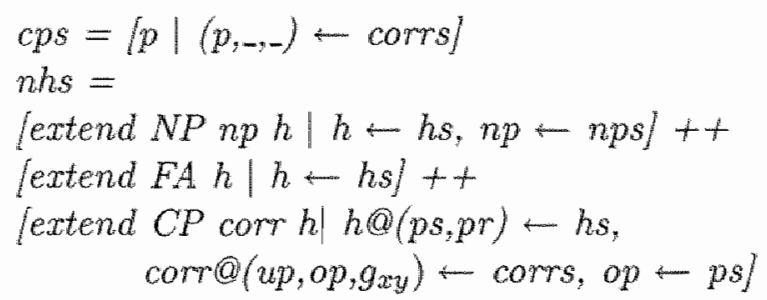

The update function extends the existing hypothesis in various ways. First a set of new plan hypotheses are created for the new observation. Secondly each existing assignment hypothesis is extended with the FA option and finally, the correlations are incorporated in the assignment hypotheses. The input for the latter extension is the set of correlations. In each correlation the original plan, op, that correlated with the new observation are retained. This is used to only update the hypotheses that have one ore more plan lypotheses that correlated with the new observation. The update function simply concatenates the lists of plan hypotheses and of assignment hypotheses in stead of applying a disjunction. This is possible due to proposition 5.5, that states that the newly created plan hypotheses and thus assignment hypotheses differ from the previous ones, that they were based upon.

The probability of the assignment hypothesis of scan $k$, to which an correlated plan is added, is calculated by the function $P_{D} g_{x y} P\left(\Omega_{k-1}\right) /\left(1-P_{D}\right)$. So it is always divided by $\left(1-P_{D}\right)$. So for $N_{D T}$ detected targets there is a division by $\left(1-P_{D}\right)^{N_{D T}}$. This is done in anticipation of the normalization of the probability as is done in the reduce function. Here the probability is multiplied by $\left(1-P_{D}\right)^{N_{T G T}}$. So after normalization, the part of the probability that is due to the old targets being undetected or detected is: $P_{D}^{N_{D T}}\left(1-P_{D}\right)^{\left(N_{T G T}-N_{D T}\right)}$. This point was earlier discussed in section 5.6.1.

The reduce function is given below.

Algorithm 9.5 reduce function.

reduce $(p s, h s)=(p s a, k h s)$

hsa $=$ hypotheses_assessment hs

$k h s=h y p o t h e s e s \_s e l e c t i o n ~ k h s a$

$\left.p s a=p s \cap|p|\left(p s_{,-}\right) \leftarrow h s a, p \leftarrow p s\right]$

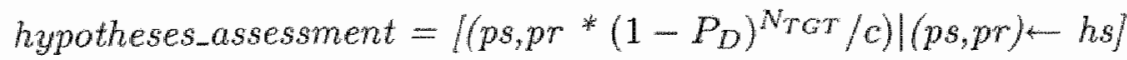


where $c=\operatorname{sum}(p r \mid(-, p r) \leftarrow h s)$

hypotheses_selection $k$ hs $=$ take $k \cdot$ sort hs

In the algorithm, the take $k$ hs function takes the first $k$ elements of the plan hypotheses list, $P_{D}$ gives the detection probability of a target. So the reduce ranks the individual assignment hypotheses of the set hs. This is done by the hypothesis_assessment function, that calculates the assignment probabilities of the hypotheses. The hypotheses_selection function reduces the number of hypotheses to the $k$-best hypotheses.

\subsubsection{Soundness and completeness}

In this sub section, the soundness and completeness of the algorithm are checked. For this the formal description of a non-hierarchical, unidentified observations plan recognizer, definition 5.3, is applied. In the figure 9.1 an attempt is made to display the sequence of processing in the Tactical Plan Recognizer.

For the first observation, 01 , the create function generates the first assignment hypotheses (in the picture indicated by the flow of $\{(o 1, f a),(o 1, p 1), \cdots\})$ also see equation 9.1 , page 156 ). If we look at the create function, it first calls the problem_definition and planning functions. The equivalence set of the set of observations consists of the first observation $\|\sigma 1\|_{h}=\left\{o_{1}\right\}$. The codomain of the assignment function, $\Psi_{h}\left(\|o 1\|_{h}\right)=\{n p 11, n p 12, \cdots\}$. The requirements relate the only observation directly with the complete set of plan hypotheses. Now discuss the soundness of the first assignment hypothesis:

1. consider the consistency condition: $\Gamma \cup \mid \sigma_{1} \|_{h} \neq \rightarrow \Psi_{h}(o 1)$. With the proper substitution, this becomes: $\Gamma \cup\{01\} \not \forall \rightarrow\{n p 11, n p 12, \cdots\}$. This property was already ascertained in sections 7.2 and 7.3 .

2. The explanation property: $\Gamma \cup \Psi_{h}(o 1) \neq o 1$, this can be rewritten as $\Gamma \cup\{n p 11, n p 12, \cdots\}$. Also this property was ascertained in sections 7.2 and 7.3 .

For the completeness it is required that the set of plan hypotheses, $\{p 1, p 2, \cdots\}$ is complete. This property goes for the problem_definition and planner functions, see the discussion in sections 7.2 and 7.3 . 


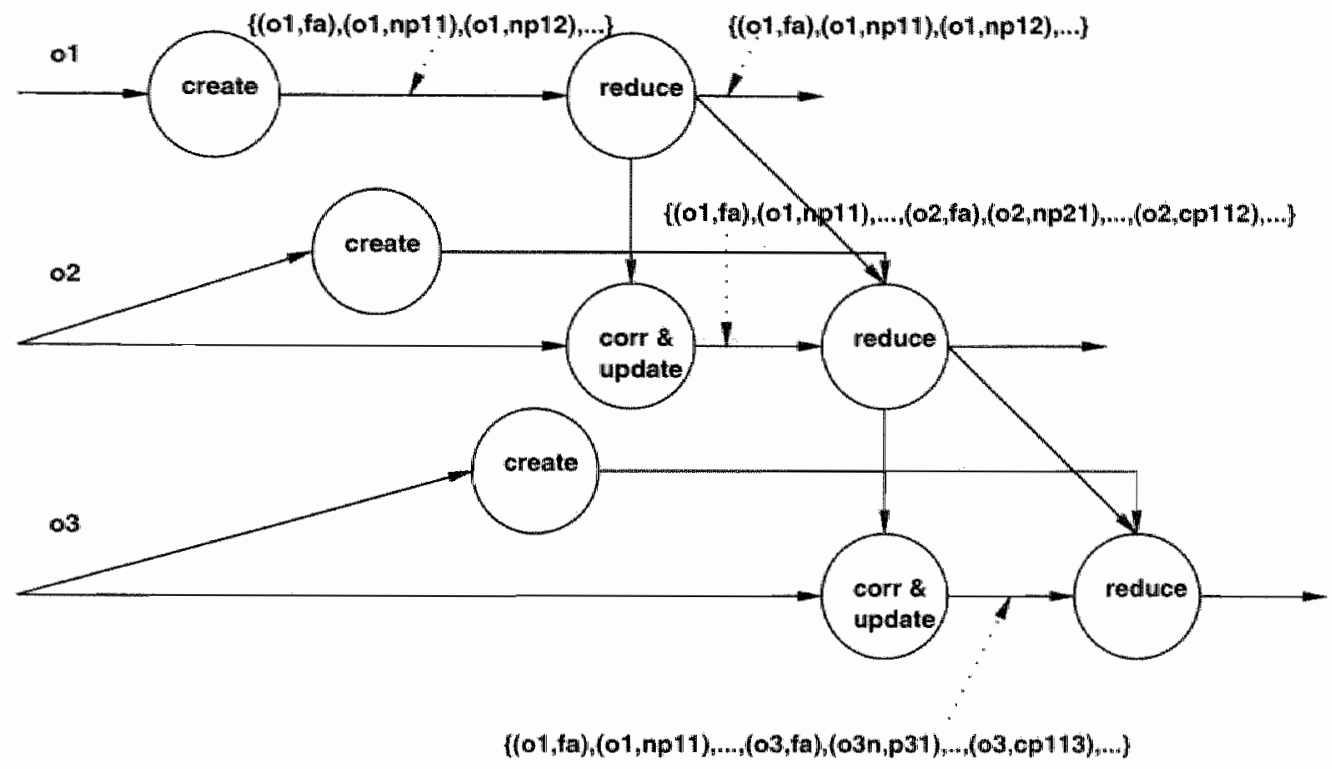

Figure 9.1: An observation-wise view of the processing in the Tactical Plan Recognizer. For the purpose of discussion of the soundness and completeness of the algorithm, in the outputs the assignments from each plot to its plan hypotheses $(o, p)$ are displayed. 
The second observation, 02 , is related to the old plan hypotheses by correlations, but also new plan hypotheses are created for this observation. The new plan hypotheses that are created for it are: $n p 2_{1}, n p 2_{2}, \cdots, n p 2_{n}$. However the set of new plans is unified with a set of updated plan hypotheses: $c p 11_{2}, c p 12_{2}, \cdots, c p 12_{m}$ (see equation 9.2 , page 157 ). The updated plan hypotheses are based on the first observation and are updated for the second observation. The parents of the updated plan hypotheses were selected from. the original set generated for the first observation, by means of the correlate function.

In the update function, the correlated plan hypotheses are created. Now check the soundness of the system sofar.

1. The consistency requirement. First consider the new plan hypotheses that will be created by the same create function that was already found to be sound and complete in the discussion above. Correlate checks whether the new observation is consistent with the parent plan hypothesis: corr $(p s, \ldots)$ o2 $=$ fupdate $^{\prime} p \circ \mid p \leftarrow p s, \cdots, p$ cw o. $)$ and its output is a effectively an assignment $\left\{\left(o 2, c p 11_{1}\right),\left(o 2, c p 12_{2}\right), \cdots\right\}$. The updating function does not affect the logical statement of a plan hypothesis. The cluster update function combines the two sets, which both are consistent with the second observation, so the combined set also has this property. This is summarized in the following equations, let nps be the set of new plan hypotheses for observation 02 and $c p s$ the set of correlated plan hypotheses, then:

update its oprs corrs ( $p s, h s) o=(n p s++c p s++p s, n h s$ )

then $n p s++c p s$ can be substituted with:

$[n p \mid n p \leftarrow n p s, n p c w$ o2 $]++|c p| c p \leftarrow c p s, c p c w o 2]$

$(p \mid p-n p s+c p s, p$ cw 02$)$

In fact $\|o 2\|_{h}=\left\{o_{2}\right\}$ and $\Psi(02)_{h}=n p s++c p s$ and given the reasoning

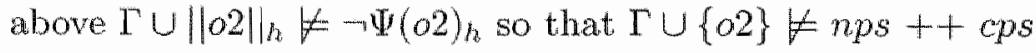

2. The explanation property. Looking at the output set of plan hypotheses, it has been proven that the set nps can explain 02 as it has been generated by the generate_hypotheses function, the plans will contain an action that will unify with $o 2$. The cps set is proven to be consistent with 02 . According to assumption 5.1, 105, the correlated plans will also contain an action that will unify with the observation $o 2$. So now the following goes: $\forall p \in c p s \cup c p s: \Gamma \cup p \models o 2$. 
The third and following observations will be processed like $o 2$ and so the same reasoning about the consistency and explanation properties goes.

With regard to completeness, again the algorithm is not complete when only the output is considered. Again, pruming removes logically sound hypotheses. If we look at the internal plan hypotheses after one scan they are partly generated by the complete generate_hypotheses function and partly consist of newly generated correlated hypotheses.

\subsubsection{Complexity}

The complexity can be calculated as is done by the Waard [93] for the tracking case. A significant difference between the Tactical Plan Recognizer and a normal tracking application is that in tracking for each observation only a false alarm and a new target option is created in the hypothesis tree, while in the Tactical Plan Recognizer $n$ plan hypotheses are created. This is a similar situation as found in the generalizations of MHT, proposed by Mulder in [53]. Here, there is not one new track hypothesis for each new plot, but several new hypotheses about the target of the plot are started and maintained. For example, for each possible class of the target a new tracli hypothesis is started and the assignment probability is augmented by a classification probability.

\subsubsection{Single sensor}

For each observation that is entered into the hypothesis tree, at least a $n$ np branches are added to the tree. But also correlations to plan hypotheses that have been initiated in previous scans in the branch to which the new branch of the new observation is entered. That is, in such a branch a plan hypothesis of previous scans can only be assigned to one observation of the new scan and plan hypotheses that also have been generated by $n p$ in the new scan are not available for assignment to the new observation of the same scan. If we assume that the number of such previous plan hypotheses available for new observation-plan hypothesis assignment, varies between 0 and $N_{T G T}$, then each observation generates between $n$ and $n+N_{T G T}$ new branches. Then the number of hypotheses given the number of observations entered in the system, $N_{O}$ (Note that $N_{O}$ may encompass various scans of $M_{K}$ observations) and the maximal number of plan hypotheses $N_{T G T}$ varies between:

$$
\begin{aligned}
N H\left(N_{O}-1, N_{T G T}\right) \cdot n & \leq N H\left(N_{O}, N_{T G T}\right) \leq N H\left(N_{O}-1, N_{T G T}\right) \cdot\left(n+N_{T G T}\right) \\
n & \leq N H\left(1, N_{T G T}\right) \leq\left(n+N_{T G T}\right)
\end{aligned}
$$


Assume that $N_{T G T}$ remained constant due to deletion of 'old' plan hypotheses that were not updated any more. So the number of hypotheses varies between:

$$
n^{N_{O}} \leq N H\left(N_{O}, N_{T G T}\right) \leq\left(n+N_{T G T}\right)^{N_{O}}
$$

and is clearly exponential with the number of new plan hypotheses that are created for each observation. In the approach above the number of correlations (assignments to previous plan hypotheses) is unconstrained.

A more exact calculation is given below and is based on that of de Waard, $[93]$ of the complexity of a init-plan hypothesis $\rightarrow$ deleted-plan hypothesis correlation system. In the figure below the structure of observation-plan hypothesis assignments and observation-np/FA assignments is given.

\begin{tabular}{|l|l|l|l|l|l|}
\hline$T_{a}$ & $\cdots$ & $T_{m}$ & $n p 1_{1} / n p 1_{2} / \cdots / n p 1_{n}$ & $\cdots$ & $n p 1_{1} / n p 1_{2} / \cdots / n p 1_{n 2}$ \\
\hline$o_{1}$ & $\cdots$ & $o_{k}$ & $o_{k+1}$ & $\cdots$ & $o_{N_{O}}$ \\
\hline
\end{tabular}

Where: $a_{i}$ is the $i^{t h}$ observation, $k \in\left[0, \min \left(N_{O}, N_{T G T}\right)\right]$. For the calculation of the complexity we assume again that there are continuously $N_{T G T}$ plan hypotheses in the system. Then the number of hypotheses with $k$ observations assigned to one of the $N_{T G T}$ plan hypotheses is calculated as follows:

1. The number of subsets of $k$ observations that can be constructed out of $N_{O}$ observations. This is the number of combinations of $N_{O}$ distinct objects taken $k$ at a time:

$$
\left(\begin{array}{c}
N_{0} \\
k
\end{array}\right)=\frac{N_{0} !}{k !\left(N_{O}-k\right) !}
$$

Note: here only one sequence of observations is considered in such a subset: the sequence in which the observations enter the system, therefore the particular sequence of the combination is of no interest.

2. Each of the observations is assigned to a plan hypothesis of $N_{T G T}$ plan hypotheses. To model all different assignments, we need to know all sequences of $k$ plan hypotheses of a set of $N_{T G T}$ plan hypotheses. Each sequence corresponds with a particular assignment. This is the number of permutations of $N_{T G T}$ objects taken $k$ at a time.

$$
N_{\text {ass }}=\frac{N_{T G T} !}{\left(N_{T G T}-k\right) !}
$$


3. The remaining $\left(N_{O}-k\right)$ observations are not assigned to an existing plan hypothesis but to one of the newly created plan hypothesis. So the number of hypotheses to be generated here is:

$$
n^{\left(N_{O}-k\right)}
$$

So if $k$ observations are assigned to a plan hypothesis, the number of hypotheses that can be generated is the product of the three terms above:

$$
\frac{N_{O} !}{k !\left(N_{O}-k\right) !} \frac{N_{T G T} !}{\left(N_{T G T}-k\right) !} n^{\left(N_{O}-k\right)}
$$

$k$ can vary between 0 until $\min \left(N_{O}, N_{T G T}\right)$. So the total number of hypotheses is:

$$
N H\left(N_{O}, N_{T G T}\right)=\sum_{k=0}^{\min \left(N_{O}, N_{T G T}\right)} \frac{N_{O} !}{k !\left(N_{O}-k\right) !} \frac{N_{T G T} !}{\left(N_{T G T}-k\right) !} n^{\left(N_{O}-k\right)}
$$

The closed form of the summation can be derived with computer algebra and is:

$$
\begin{gathered}
N H\left(N_{O}, N_{T G T}\right)=\frac{N_{O} ! N_{T G T} ! n^{N_{O}-1} F\left(\left[1,-N_{T G T}+1,-N_{O}+1\right],[2], n^{-1}\right)}{\left(N_{O}-1\right) !\left(N_{T G T}-1\right) !}- \\
\frac{N_{O} ! N_{T G T} ! n^{N_{O}-1-N} F\left(\left[1,-N_{O}+N+1,-N_{T G T}+N+1\right],[2+N], n^{-1}\right)}{(N+1) !\left(N_{O}-1-N\right) !\left(N_{T G T}-1-N\right) !}
\end{gathered}
$$

Where $N=\min \left(\left(N_{O}, N_{T G T}\right)\right.$ and $F(n, d, z)$ is the generalized hypergeometric function. $F$ is also known as Barnes' extended hypergeometric function, $n$ is a vector of $\mathrm{j}$ coefficients and $d$ is a vector of $\mathrm{k}$ coefficients.

\subsubsection{Multi Sensor}

Of course, the multi sensor case is more complex than the single sensor case. Contrary to the single sensor case, in which the number of plan hypotheses only increases by new plans, while the number of plans that are open for correlation remains fixed on $N_{T G T}$, in the multi sensor case, the number of plans, open for new correlations, increases with each correlation. 
First look at the number of parenthood constraints that occur. In Kruger's master's thesis, [45], the number of parenthood constraints in the hypotheses tree is calculated for $N_{O}$ observations and $N_{T G T}$ plan hypotheses to be:

$$
\left(2 N_{O}-1\right)+\left(N_{T G T}+1\right) 2^{N_{O}-1}
$$

This can be translated to our plan recognition case. Let $n$ be the number of new plan hypotheses that are created for each new observation, then the number of parenthood constraints between plan hypotheses is given by:

$$
\left(n N_{O}-1\right)+\left(N_{T G T}+1\right) n^{N_{O}-1}
$$

Now consider the complexity of the hypothesis tree. In equation 9.6 the factor $n+N_{T G T}$ indicated the maximal number of new branches due to a new observation. In the multi-sensor case, this number increases with the number of correlations with the previous observation: $N_{\text {corr }}$. Assume this is a fixed number. Then the number of new branches becomes: $n+N_{\text {corr }}+N_{T G T}$. The range of the complexity of the hypothesis tree now is:

$$
n^{N_{O}} \leq N H\left(N_{O}, N_{T G T}\right) \leq\left(n+N_{\text {cor } x}+N_{T G T}\right)^{N_{O}}
$$

Also in Kruger, [45], it is shown that a single observation-plan hypothesis assignment problem can be rewritten as a three dimensional assignment problem. Three dimensional assignment problems are NP-complete. 


\section{Summary of part III}

In part II, the formal properties of plan recognition and the task analysis of both traditional and Tactical Plan Recognition were given. In this part, the results of the task analysis, the two Generic Task Models, have been elaborated by the design of two algorithms, one for each problem.

The design starts with an examination of suitable algorithms for the goal recognition sub-task(that is the most important sub-task of the problem definition task) and the (re-)planning task. These tasks occur in both traditional and Tactical Plan Recognition. The knowledge incorporated in the goal recognition task is modelled by a forward-chaining algorithm.

For the planning task, three plan template representations and corresponding algorithms have been discussed. The most powerful representation for plan templates are the STRIPS operators. However, the STRIPS planning algorithm is found to be unsuitable for plan recognition and particular Tactical Plan Recognition. A more suitable representation is, like Azarewicz's singleagent plans, a Deterministic Finite Automaton. The last representation, a sequence of actions, is attractive due to its simplicity. But the sequence of actions can not model all uncertainties that are found in the military world.

After discussing the basic functions, one algorithm for recognition of non hierarchical plans for identified observations is discussed and one for unidentified observations. These algorithms are intended to implement their corresponding Generic Task Models, that have been defined in the previous part. The formal properties: soundness, completeness, complexity and termination are examined for the proposed algorithm.

Again the traditional plan recognizer's algorithm is the bases for the Tactical Plan Recognizer's algorithm. The latter case is far more difficult. 


\section{Part IV}

\section{The Demonstrator and conclusions}




\section{Chapter 10}

\section{The Demonstrator}

In the previous part, algorithms for identified observations and unidentified observations are presented and their properties are discussed. The text of the previous part still is quite 'high level', and only touches the most important parts of the Tactical Plan Recognizers. Also much attention has been paid to the description of properties of these algorithms. However, the described algorithms only concern a few lines of code, the algorithms contain some simplifications.

To construct a 'real' Tactical Plan Recognizer, that actually works, much more software is required. Software for simulating the outside world and generating some of the input of the Tactical Plan Recognizer: observations and a description of the geographic situation. Many supporting functions of a plan recognizer itself are not yet mentioned in part III. Finally, one needs software for displaying the output of the Tactical Plan Recognizer for the human operator of the system. The output data is fairly complex: assignment hypotheses and plan hypotheses. So one has to examine methods of displaying it efficiently by means of a Human Computer Interface. The user interfaces presented in this part give some first ideas for this.

Given the 'real' Tactical Plan Recognition system (for unidentified observations), the performance of the algorithm is of paramount interest. For this, two scenarios are simulated, observations are generated and are input to the Tactical Plan Recognizer. The outputs are evaluated 'manually' by means of the Human Computer Interface of the Simulator. Some performance measures are introduced.

As mentioned before, the models for the military operations introduced 
here are not complete. Some parameters of the model are only guessed. One might attempt to add more details to the model, and this approach is found in the area of tracking. However, an other approach is to consider the guessed parameters as 'tunable' parameters. By an informed (and 'manual') search of the state space of these parameters, the performance can be improved to a sufficient level. In the two examples, the initial performance is sometimes not very good, but by means of tuning the Tactical Plan Recognizer, the performance is quickly improved.

The Simulator and software system presented here, can be qualified as an early Demonstrator. The term Demonstrator is used in the software industry as the outcome of a feasibility study and is a real world software system. If the Demonstrator can perform the required tasks and shows some promise, the feasibility study is considered successful. The Demonstrator precludes the development of an actual new product.

After this description of the Demonstrator and its good functioning, the thesis can be concluded. In the last chapter on conclusions, the problem statement is revisited. The various elements of this statement are recalled and the manner in which they have been addressed and the amount of success of this are discussed.

This research was a first step towards a possible development of a real Tactical Plan Recognition product, that may be a task of future Command and Control systems. But to achieve the goal of a complete 'real world' product some work still remains to be done. Therefore the thesis ends with an overview of future work.

\subsection{The architecture of the Demonstrator}

For the showing the feasibility of the Tactical Plan Recognition algorithm of part III, a Demonstrator has been developed and its organization will be presented in this section. The Demonstrator is a fairly large piece of software. It functions 'off-line', no provisions for real time processing have been made. It consists of the following three parts:

1. Tactical Plan Recognizer: a Haskell program for the tactical plan recognition processing and

2. Environment Simulator: a Java ${ }^{1}$ program for simulating the environ-

\footnotetext{
${ }^{1}$ Java is a trademark of Sun Microsystems.
} 


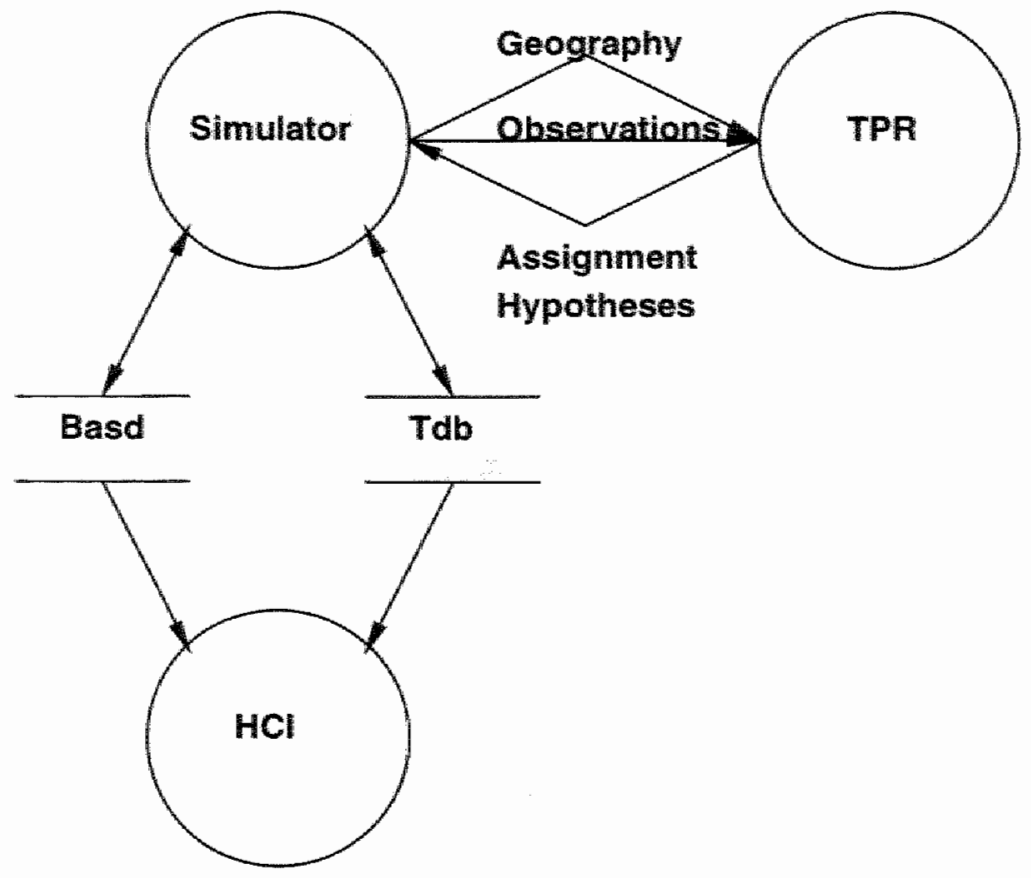

Figure 10.1: The data flow diagram of the Demonstrator environment.

ment and generating observations and, finally,

3. The Human Computer Interface (HCI): a display the geographical situation and the output of the Tactical Plan Recognizer and overall control by the operator.

The Simulator is concerned with generating data for a scenario. A scenario describes a particular military operation. An example of a scenario is found in the army example. Here we have a geographic environment (in this case only the front-line) and various friendly and non-friendly military units and helicopters at their geographic locations. But the scenario also contains a dynamic part. So the Simulator has to 'execute' the movements and actions of the targets. For this, it has know the operational plan that is executed. The plan consists of the object's actions and the object's trajectory. Some of the military units that are modelled by the Simulator are friendly observers and the Simulator has to generate observations of these, when a target enters their coverage. These observations are input the to Tactical Plan Recognizer. 
The Tactical Plan Recognizer, off course, only has knowledge of its friendly units and the generated observations.

A data flow diagram of the Simulator and Tactical Plan Recognizer is given in figure 10.1. The data flows concern 1) geographic information generated by the Simulator and that is send to the Tactical Plan Recognizer, 2) the generated observations of enemy targets that are also send to the Tactical Plan Recognizer and 3) assignment hypotheses that are generated by the Tactical Plan Recognizer and send to the Simulator for display. The Tactical Plan Recognizer itself has knowledge of the enemy doctrines (by means of fixed sequence plan templates) and combines it with the geographic information. Then it outputs the assignment hypotheses that might explain the observations. The assignment hypotheses are communicated to the Simulator for display in the Human Computer Interface. Then the hypotheses can be examined by the operator.

The data flows between the Tactical Plan Recognizer and the simulation program are implemented by the following data files:

1. Geagraphic information: the geographic information is communicated from the Simulator to the Tactical Plan Recognizer by a '*.bsd' file. This is an ASCII text file that lists the characteristics of all the geographic objects and units in the scenario.

2. Observations: the Simulator generates a set of Observations and communicates them to the Tactical Plan Recognizer through a '*.obs' file. This is an ASCII text file that simply lists the observations.

3. Assignment Hypotheses: the Tactical Plan Recognizer reads the geographic information and the observations from the files that were generated by the Simulator. It then, sequentially, enters the observations in the plan recognition algorithm. The output of assignment hypotheses is stored in a '*tpr.xml' file. 'The XML schema is given in appendix C.

In general, Simulators of a military scenarios, have to work with a number of related objects. These objects are best organized in databases and these Simulators always maintain a number of databases. The databases are the backbone of the Simulator and contain all information. The processing of the Simulator reads information from the databases and stores its results there. The advantage of this database approach is that no, globally relevant, information is retained locally in algorithms. All information is globally available 
in the databases and various function can communicate complex information by means of the database content.

Our Simulator operates upon two simple object oriented databases ${ }^{2}:$ 1) the Track Database (Tdb) containing plan hypotheses and assignment hypotheses and 2) the Basic Database ${ }^{3}$ (Basd) containing information about geographic objects that play a role in the tactical world. When the system starts, the Basic Database already contains all scenario information and can generate geographic data and observations for each scenario. The Tactical Plan Recognizer then is invoked manually and generates the XML file. This XML file is input for the Simulator and the Simulator translates the XML data to internal objects and stores them in the Track Database. The Human Computer Interface simply examines the databases and displays the contents of both databases in an appropriate manner.

The software engineering design of the main functions: the Simulator, the Human Computer Interface and the Tactical Plan Recognizer, are further explained in the following sections.

\subsection{The design of the Simulator}

Pivotal to the workings of the Simulator and HCI are the databases. The 'Basic database' stores geographic information and information about the military objects and military units. For each type of object that can occur in the scenario, a Java class is defined. Relations between classes are modelled in an object oriented manner with pointers and lists of objects. The basic database software, that is its sensors and observer objects, can also generate observations given a scenario and target and output them in the 'w'obs' file. The 'track database' reads the XML output file of the Tactical Plan Recognizer and translates it to objects that are stored in the Track Database. The contents of the databases are displayed by the HCI.

These object oriented databases contain, off course, objects that are instances of classes. In our software, we find the following types of classes:

- First, there are 'low level' classes that directly model objects. In the Ba-

\footnotetext{
'These simple databases are can also be called stores, they don't provide persistence of data or sophisticated mutations and queries in a query language. But they do organize the objiects and their extension.

"The name 'Basic Database' was already in use in the DFD project and was retaned in this research. A more appropriate name is Geographic Information System (GIS).
} 
sic Database one finds classes such as lines, targets, but also sensors. In the Track Database, one finds classes like scans, assignment hypotheses, plan hypotheses.

- Secondly, there are database objects. In these the extensions of the previous classes are organized. This concerns the Tdb and Basd classes.

- Finally, there are exceptions, the classes TdbException and BasicException.

The classes of the Track Database and Basic Database are elucidated in detail in the following subsections.

\subsubsection{Class diagram of the Simulator's Basic Database}

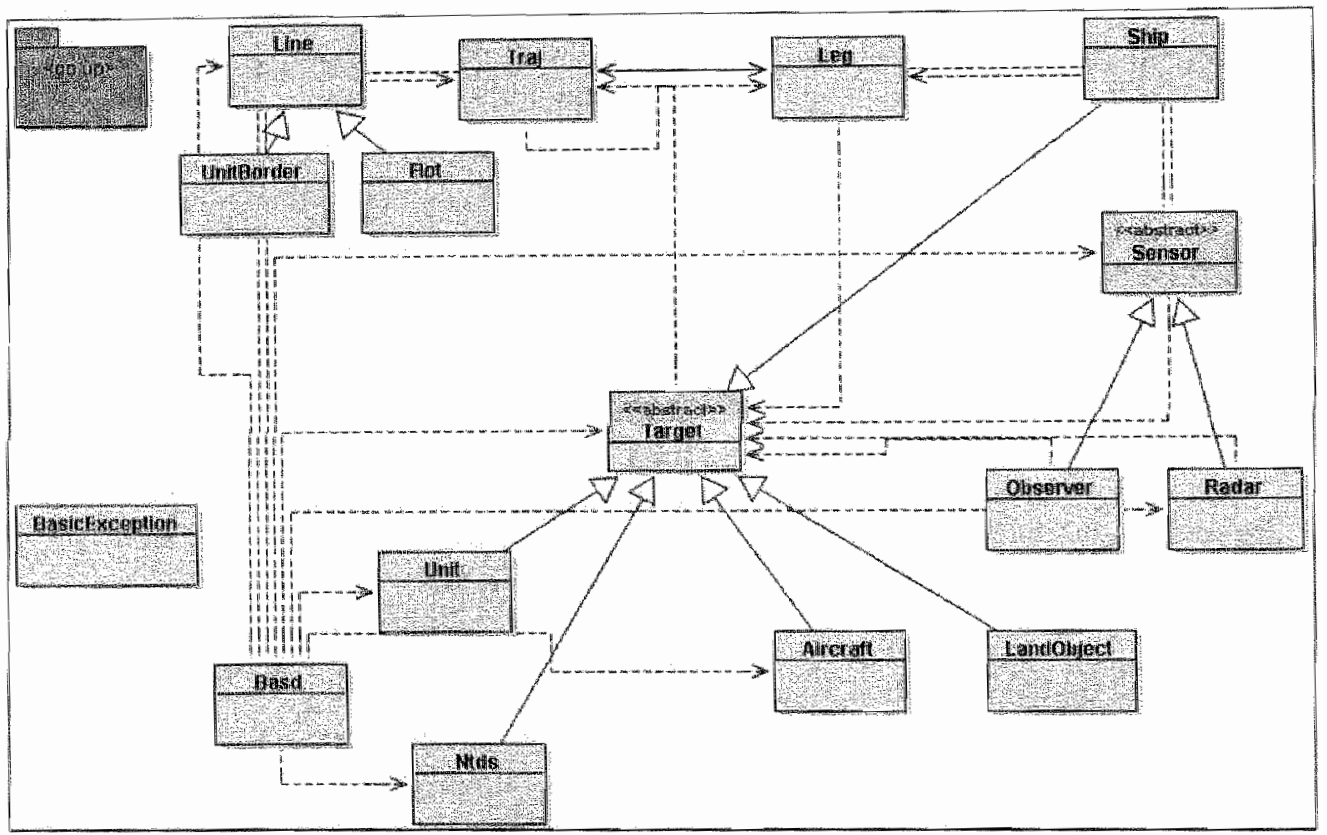

Figure 10.2: The UML class diagram of the Basic database (Basd).

The information model of an object oriented database is generally graphically displayed by means of class diagrams. These depict the classes and their relations in an abstract manner. In this sub section some of the class diagrams 
of the databases are presented in an Unified Modelling Language (UML) type notation. The class diagrams are actually be generated from the Java byte code, by means of its introspection features.

The Basic database (Basd) class models the enviromment. Here one finds 'real' objects such as targets, units, border lines and aircraft. The Basic Database's class diagram is found in figure 10.2. The Basic Database itself also is a Java class. It is the access point for the extensions of the actual objects that are modelled. These objects are themselves also implemented by means of Java classes (even if one wanted to, there is no other option for this in Java). The 'real' object classes are: abstract lines implemented by the Line class. Its sub-classes are linked by a solid arrow in the picture to the UnitBorder and the Flot (Forward Line Own Troops, i.e. the front-line) classes. Another abstract class is a Target. Its sub-classes are Units, Aircraft, LandObjects and Ships. The last abstract class is the Sensor. Its subclasses are the Observer and the Radar. There may seem to be just a few classes, but for our Demonstrator and its scenarios, this description of the environment suffices.

Apart from sub-class abstraction relations, one also finds composite relations: part-of relations. These are indicated by dashed arrows. In the Basic Database, the Target may have a Trajectory that models the movement of the target. The Trajectory class consists of a number of Legs: straight line motions of the target. As all these classes are stored in the Basic Database object, they are also a part of that database.

Finally the Basic Database contains an exception class that may be invoked by the methods of the objects in the Basic Database.

\subsubsection{The class diagram of the Simulator's Track Database}

The Track Database class (Tdb) stores the results of the Tactical Plan Recognizer assignment hypotheses and plan hypotheses and the input of the Tactical Plan Recognizer, that is the observations and the geographical situation. The Track Database also stores the measurements and results of a tracking process, but this functionality was not required for the Demonstrator. The class diagram of the track database is found in figure 10.3.

Whereas the Basic Database is fairly straightforward, the Track database merits some explanation. Again there is a database object that organizes the extension of the other information entities. As we know, assignment hypotheses, $\Psi_{h}$, are the most important information entity. These are modelled by the 


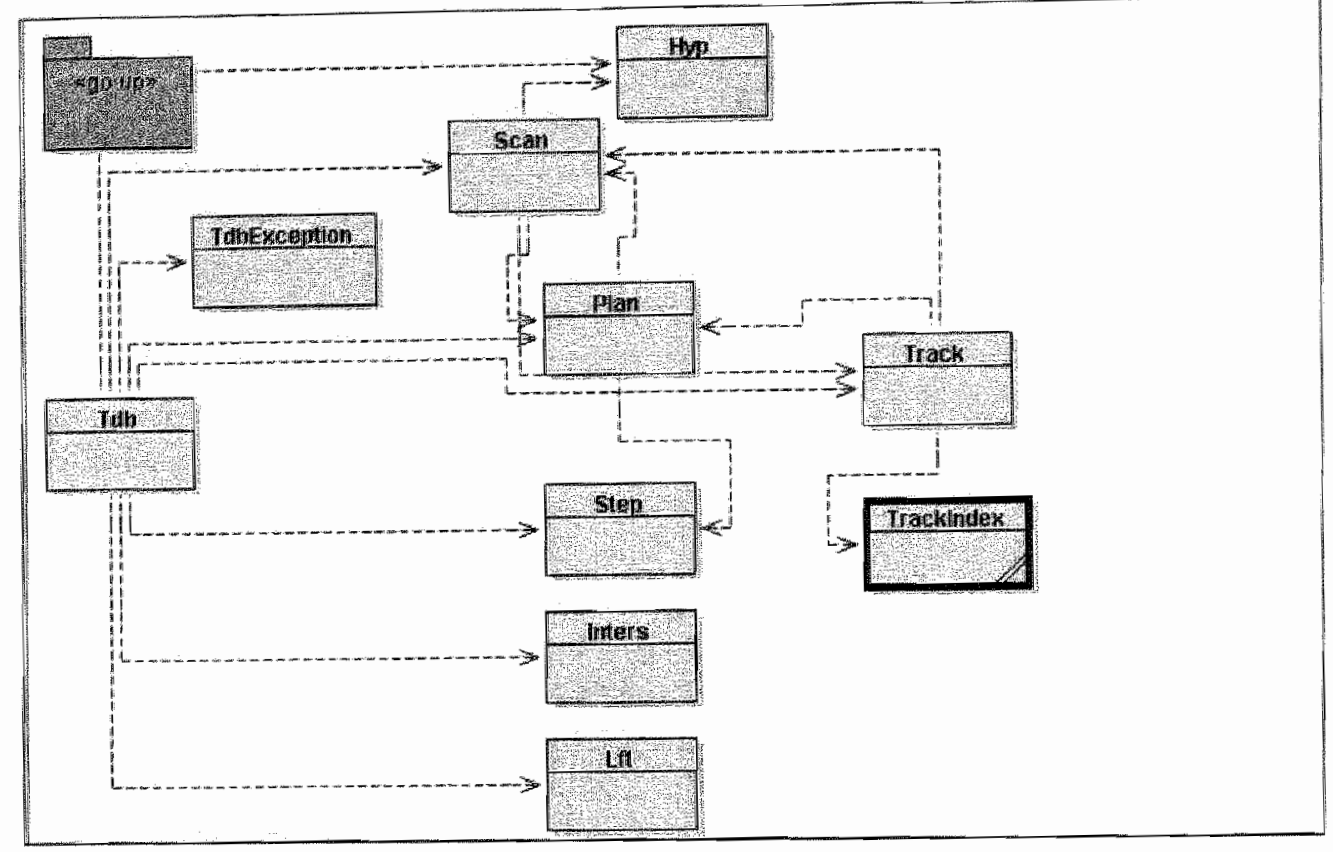

Figure 10.3: The UML class diagram of the Track database (Tdb).

Hyp class, found at the top of figure 10.3. For each scan, we have a number of assignment hypotheses, scan $=\left\{\Psi_{h}\right\}$, that are based on previous assignment hypotheses and are based on new observations that are entered into the plan recognizer in that scan. Therefore the Hyp class is a part of the Scan class. An assignment hypothesis contains a set of plan hypotheses $\Psi_{h}=(\{\phi\}, p)$ (where $\{\phi\}$ is a set of plan hypotheses and $p$ is the hypothesis' probability), that are modelled by the class Plan and a probability. But in order to add identification numbers these plan hypotheses, they are wrapped in a Track class, that also maintains unique identification numbers: keys of the track. These keys are organized in the TrackIndex Object. In fact the main key of a track is the 'root id' that is unique (as was stated by proposition 5.2). By means of the Track objects, we can organize successive plan hypotheses over several scans. Remember that an plan hypothesis object is only a correlation option of a previous plan hypothesis to a new observation, or concerns a new plan or false alarm. The estimation of a particular objects behavior during the observation time, is given by the successive plan hypotheses organized in 
the tracks. Thus the Track class gives an overview of the plan hypotheses of the target as it is estimated from observations over a period of time.

\subsubsection{The numerical mathematics module}

An other interesting ${ }^{4}$ module concerns some numerical mathematics functionality that is available in the Nrc object. The UML class diagram is given in figure 10.4. The Nrc class contains some static methods for some mathematical functions. The NewtonRaphson and Bisection abstract classes implement the corresponding function root-finding algorithms. The roots of a function are the values of the domain that correspond with a zero function value: $x_{i}$ such that $f\left(x_{i}\right)=0$, where $x_{i}$ are the roots and $f(x)$ denotes the function. They are abstract classes, the function at hand is defined as an abstract method. The instances of the abstract classes implement the actual function, thus communicating this function the the root finding algorithm.

\subsection{The design of the Human Computer Interface}

The Human Computer Interface is implemented with Java's Swing library and facilities for an operational display. The program can either be displayed in a Java applet ${ }^{5}$, or can run as a stand-alone Java program.

The Simulator's interface consists of three parts. In figure 10.5 and figure 10.6 examples of the complete user interface are displayed. The display consists of the following three parts (from the left to the right): 1) an operational display, giving the geographic situation of the scenario at hand, 2) a (rather slim) panel: the operational control panel that controls the simulator and other displays and 3) the plan information display that displays the contents of the Track Database in a alpha numerical manner.

The displays are described in more detail in the following sub sections.

\subsubsection{The operational display}

'This consists of a tabbed display with two talos marked 'Ops' and 'T-W'. The first tab, 'Ops', gives the displays the geography and the location of various

\footnotetext{
At least for readers that are interested in object oriented programming.

${ }^{5}$ A Java Applet can be included in a HTML document and can be displayed in a HTML viewer, such as Microsoft's Internet Explorer. The displayed applet is interactive for the user.
} 


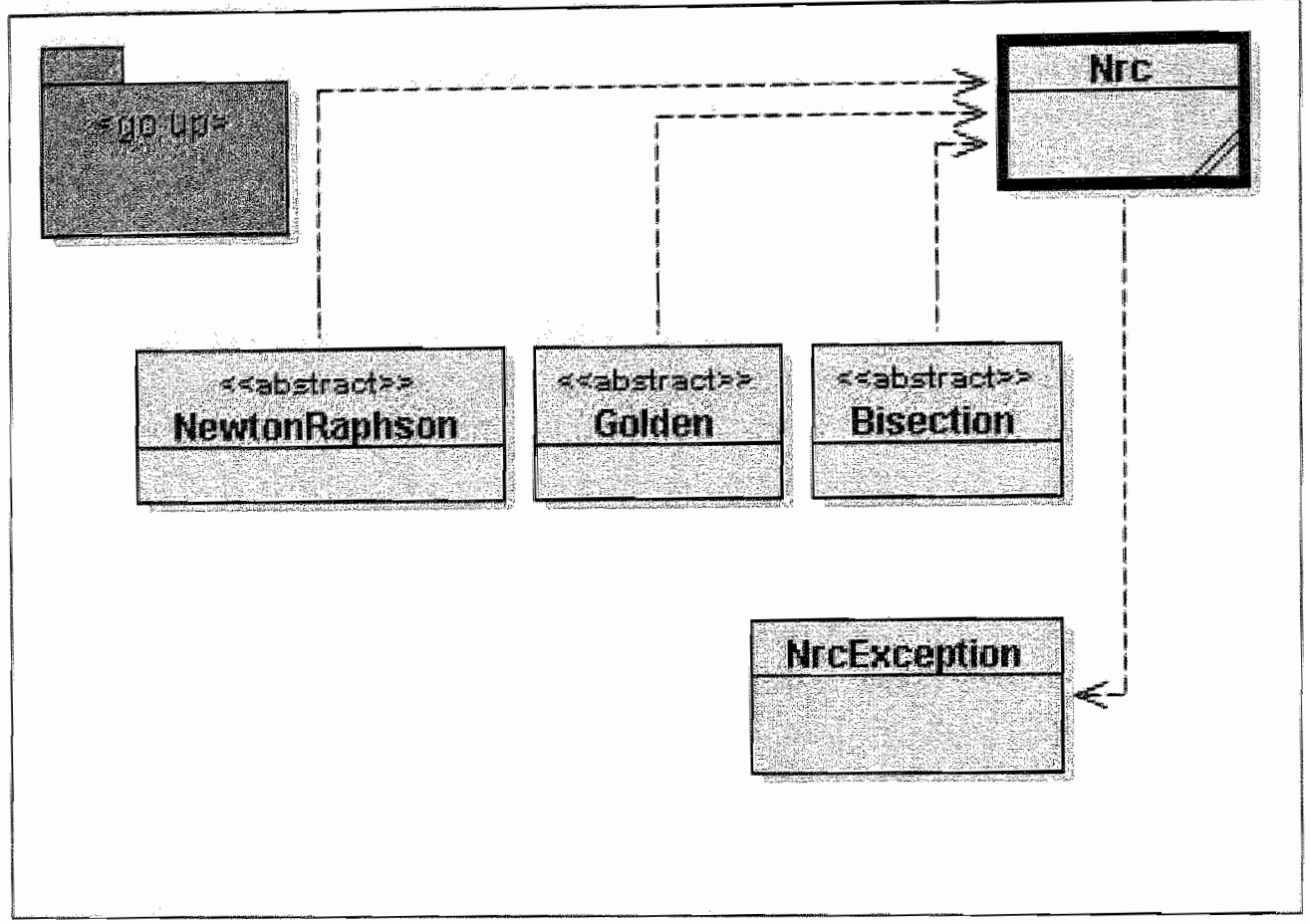

Figure 10.4: The UML class diagram of the numerical mathematics objects.

units, targets and geographic objects. See the left graphical window of figure 10.5. A detailed screen dump is displayed in figures 10.11 and 10.13. The first picture shows the geography as the background. It also displays the position and type of military units U1 till TK5. They are displayed by military icons. The range and center of the operational display is controlled by drop-down menus, located at the top bar.

The second tab, "T-W", gives a Time distance diagram: it displays the time-distance diagram of the trajectory of the selected plan hypotheses. The time distance diagram is generally used to display the movement of an object along legs. Horizontally the time is displayed and vertically the distance made by the target along the leg. In our plan recognition case, the time distance diagram is based upon estimates the the starting point of time of movement of the target along a leg and the estimated speed of the target.

For an example of a time distance diagram, see the graphical window on 


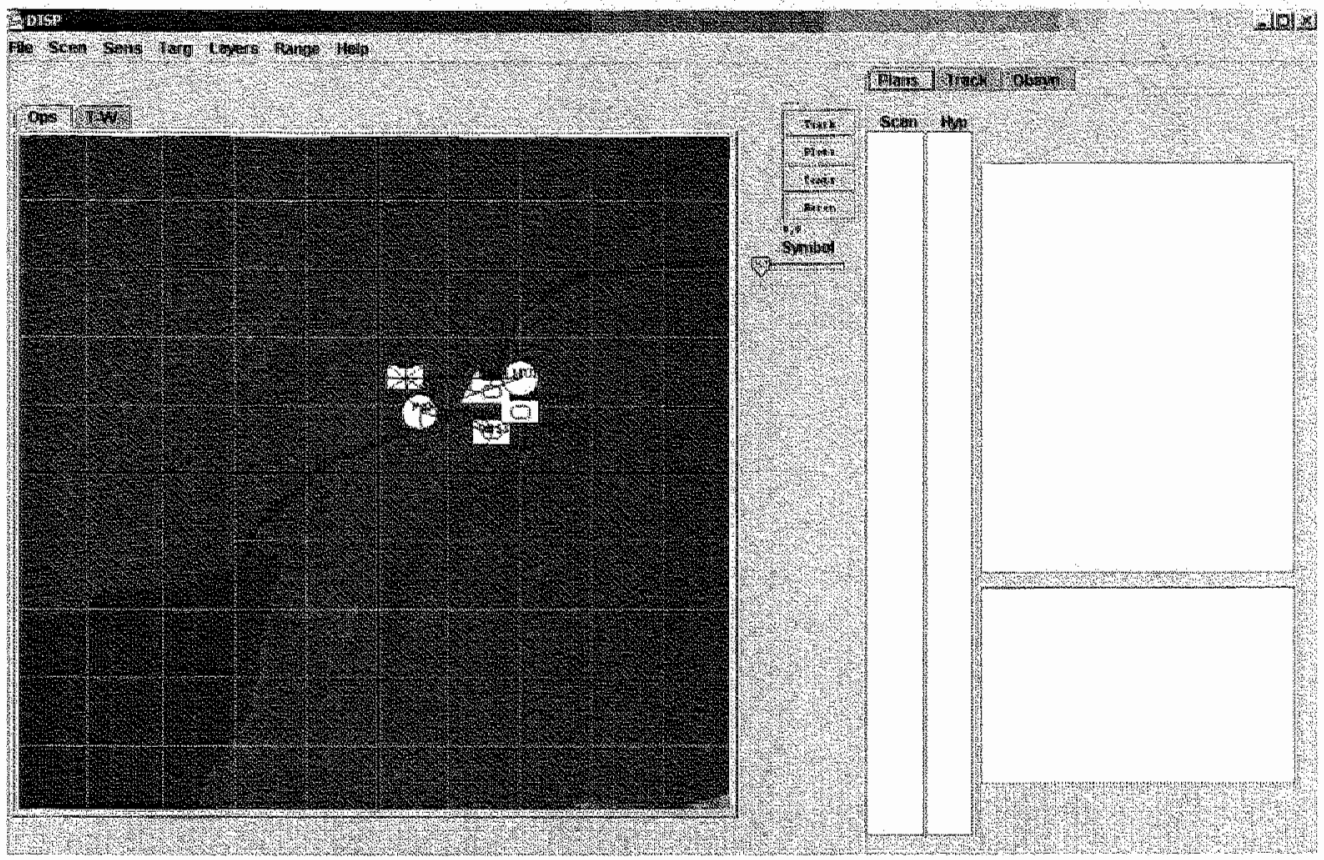

Figure 10.5: 'The user interface of the Demonstrator, with on the left part the operational display displaying the geography of a scenario, in the middle the operational control panel and to the right the plan information display.

the left of figure 10.6. A detailed screen dump of a time distance diagram is given in figure 10.10. It the showed graph has a saw-tooth appearance. Each 'tooth' corresponds with the movement along one leg. The display is rather simple due to the limitations of the graph-drawing program.

\subsubsection{The operational control}

Here the user can perform scaling operations on the operational display and invoke the simulation. At the top of this center display the name of the current scenario is displayed. Then there are several buttons. The first button, that is labeled 'Track', displays tracks, but this is not relevant for this research. The next button, labelled 'Plots', generates plots and observations of the current scenario. These are stored in an "冰.obs' ASCII file and can be input of the Tactical Plan Recognizer. The third and fourth buttons, 'Centr' and 'Recen, 


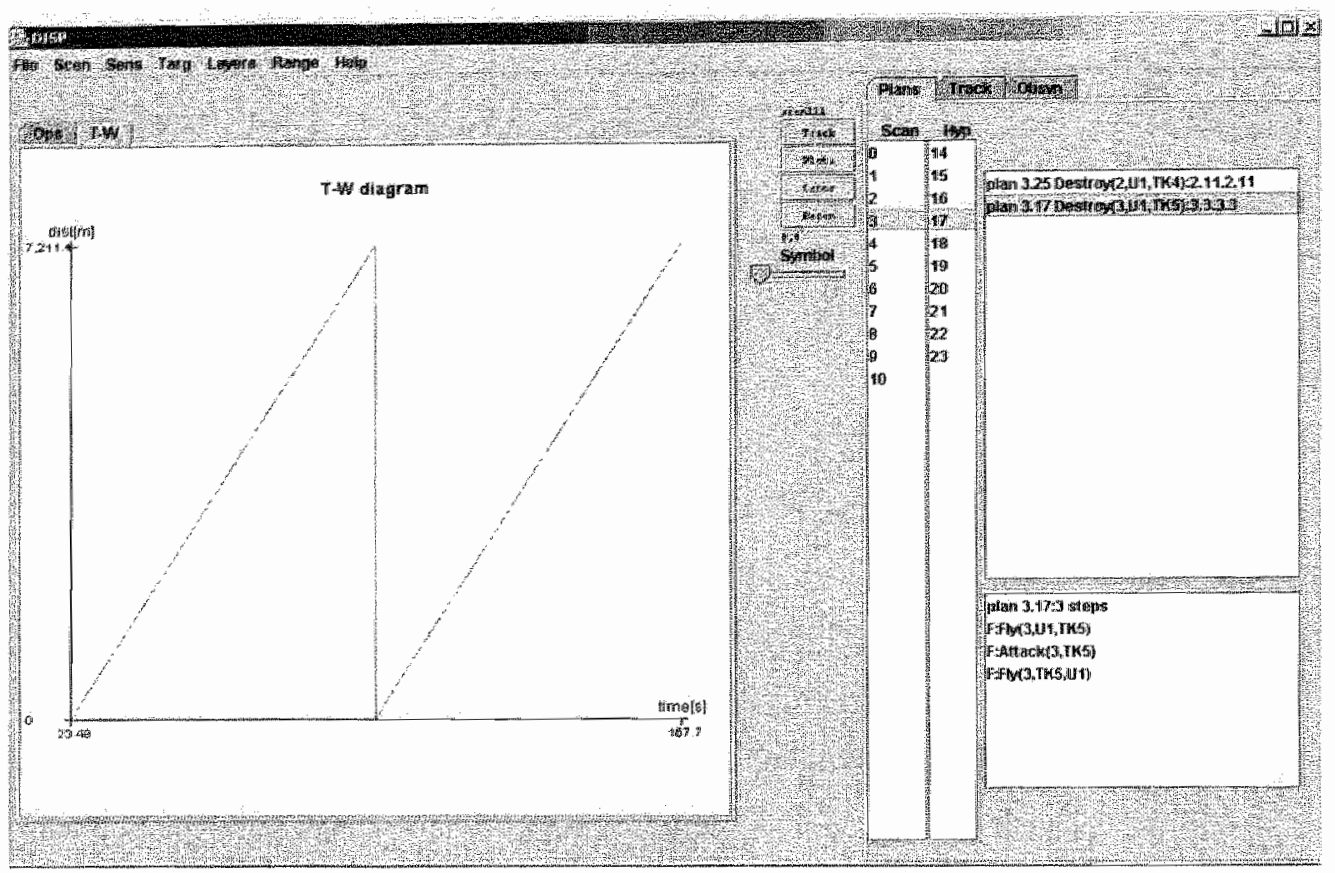

Figure 10.6: The user interface of the Demonstrator, with on the left operational display the time-distance diagram.

control the centre of the operational display. The bottom control is a slider bar that controls the size of the icons that represent targets on the operational display.

\subsubsection{The plan information display}

The plan information display consists of three tabs, 'Plans', the assignment lypothesis oriented display, "Track', the track oriented display of plan hypotheses and 'Obsvn', the display of the set of observations. The three displays are elucidated in detail in the following sections.

1. the first tab, 'Plans', concerns an assignment hypothesis oriented display. An example screen-dump is given in figure 10.7. Here, for a particular scan, its assignment hypotheses' identification numbers are displayed by the left two text windows. The assignment hypotheses are displayed from top to bottom in order of decreasing probability. 
The user can select a particular scan by clicking on the scan number. The line then turns grey. As soon as the scan number is clicked, the set of hypotheses of the scan is displayed in the 'Hyp' text window. After clicking an assignment hypothesis' identification number, the plan hypotheses of the assignment hypothesis are displayed in the upper right text window. Finally clicking on one plan hypothesis results in the display of its steps in the lower right text window.

In the figure assignment hypothesis number 17 of scan number 3 has been selected by the user. This selection is indicated by the grey background of the lines. The assignment hypothesis consists of two plan hypotheses: numbers 3.25 and 3.17 . The first plan concerns an object with identification number 2 . The identification has been generated by the Tactical Plan Recognizer, and was not available on forehand. The second plan concerns an object with identification number 3 . A displayed plan consists of the plan id, e.g. 3.25, than the text of the goal of the plan, for plan 3.25 it is: 'Destroy(2,U1,TK4)', and finally the internal track number, 2.11.2.11. The second plan, 3.17, has been clicked upon by the user and its contents are displayed in the lower right text window. The plan consists of three steps: F:Fly (3,U1,TK5), F:Attack(3,TK5),F:Fly (3,TK5,U1). The 'F:' label indicates that the actions will occur in the future. There are two other labels: 'D:' indicates that an action has already has been executed and 'C:' indicates that the action is being executed at the time of the scan.

2. The second tab, 'Track', invokes a track oriented display. An example screen-dump is given in figure 10.8. Here on the left is a text window with available track numbers. Clicking on a track number invokes the display of the best plan hypotheses of the track, one for each scan. In the figure, the selected track has track number 2. The last best plan hypotheses number 10.202 has been clicked upon by the user and its actions are displayed in the lower right window: F:Fly $(3, \mathrm{U} 1, \mathrm{TK} 5)$, F:Attack(3,TK5), F:Fly(TK5,U1).

3. The third tab, 'Obsvn', displays the set of observations of the current scenario. A screen-dump is found in figure 10.9. Here, the 'pid' is the plot number, 'ti' is the observation time in seconds, ' $x$ ' and ' $y$ ' give the $\mathrm{x}, \mathrm{y}$-position in kilometers and $\mathrm{h}$ gives the heading in degrees. 


\subsection{The design of the Tactical Plan Recognizer}

The Tactical Plan Recognizer itself consists of a Haskell program. The program is run in the WinHugs environment [42]. There are other Haskell environments, but because it is an interpreter, it is ideally suited for rapid prototyping. It can be run from a command window, but a nice window based Interactive Development Environment (IDE) is also available. The user interface of the Hugs IDE is shown in figure 10.14 .

The Tactical Plan Recognition program consists of the following Haskell modules:

1. Matrix: that implements basic matrix operations:+ (matrix/vector addition), -(matrix/vector subtraction), /(division by a scalar), inprod (the inproduct of two vectors), vecprod (matrix-vector product), prod (matrix-matrix product), det (matrix determinant), inv (matrix inverse) ,norm2 (vector square norm). The implementation of matrix operations is rather different from the implementation in traditional languages, because matrices are implemented with 'lists of lists'. The elements of the matrices cannot be accessed directly, and this type of matrix algorithms are called 'Indexless' matrix algorithms.

2. Filter: implements the filter operations and uses the Matrix module. It exports the following functions: initiate (state vector and covariance matrix initiation by first measurement), predict (predict the state vector and covariance matrix over a period of time), innovation (calculate the innovation between a measurement and a predicted state vector and covariance matrix), update (update a predicted state vector and covariance matrix with a measurement), distance (calculate the statistical distance(more precise the Mahanalobis distance) between a measurement and a state vector) and gate (check whether the statistical distance is in a. $95 \%, 99 \%$, etc. range).

3. Var: implements substitution of logical variables. It exports the symbols and data structures that are used in the TPR program. One of the data structures is the 'Subs' class that is used to implement substitution functions ${ }^{6}$. The substitution function takes as input a sentence with

\footnotetext{
"This refers to substitution of logical variables by other variables or literal symbols, given a set of bindings: e.g. $F l y(x$, Heathrow $) /\{x=K L 174\}=F i y(K L 174$, Heathrow $)$. Here is the substitution operator and $\{x=K L 174\}$ is a set of bindings.
} 
variables and a set of bindings outputs the same sentence, but with the variables substituted with elements of the set of bindings.

4. GIS: the Geographic Information System (GIS). This implements varous 2-D geographic queries and predicates. Some of these are: duration (calculation of the initial duration of flying along a trajectory), distance 1 (calculation of the distance of the observation to the leg), distance. (calculation of the distance projected on a leg) and direction2 (calculation of the direction of a leg in arithmetic azimuth).

5. Steps: implements queries on the actions of a plan. Some of these operations are: end_times (calculate the end times of the execution of the actions), begin times (calculate the begin times of the execution of the actions), distances (calculate the distance between end and start point of the actions), durations (calculate the durations of the actions), comp_times (compile the begin,duration and end times of the steps), begin_time_step (calculate the begin time of an action given a new observation), curnent_step_t (find the current action being executed at a particular point of time), current_step_p (find the current action being executed given the current position of the object executing the action).

6. Dfa: implements DFA planning operations and uses the Steps module. Its operations axe: plamer (generates all possible plans given initial facts, the DFA plan templates, the possible goals), begin_time_plan (find the plan's begin time given the first observation of the plan's object), update-plan (update the steps of the plan with a new observation), predict_plan (predict the kinematic state, state vector and covariance matrix, of the plan's object to a particular point of time), consistent_plan (check whether a new observations is consistent with an existing plan).

7. TPR: contains the main Tactical Plan Recognition functions. This module uses the DFA and XML modules. It implements the main functions of the Tactical Plan Recognizer. Its functions are: plan_recognizer (the main function that is exported to the Main module), hypotheses_management, update_hypotheses and generate_hypotheses, hypotheses_assesment and problem_definition.

8. Main: this function gives the main loop of the program. It calls input/output functions and processes input data by calling the TPR functions. 
Main call graph of the Haskell TPR program modules is given in figure 10.15 . The estimation of the points of time of a hypothetical plan has not been addressed yet in previous chapters. Apart from knowing the actions of hypothetical plans, the plan recognizer also has to estimate the begin and end points of time at which these actions are expected to be executed. From these estimates, the actions are labelled ' $F$ ' (to be executed at a future point of time), ' $\mathrm{C}$ ' (Currently being executed) and ' $\mathrm{D}$ ' (already executed), which are displayed in the plan information display. With regard to this calculation, there is (of course) a different approach for 'normal' actions and the movement along a leg. For the legs, a time-distance diagram, based on an estimated speed of the target, is maintained by the plan recognizer. When the first observation of a new plan hypothesis is entered into the plan recognizer, it first determines which action is being executed. From the current position and a guessed speed, the begin point of time of the legs are calculated forward for future actions and backward for already executed actions. The begin of the execution of the first leg or action, also gives the estimated begin time of the plan execution. All these estimated points of time are recalculated for each new observation that supports the plan hypotheses.

Now the Simulator has been presented, some experiments with the Simulator and Tactical Plan Recognition program will be discussed in the next chapter. 


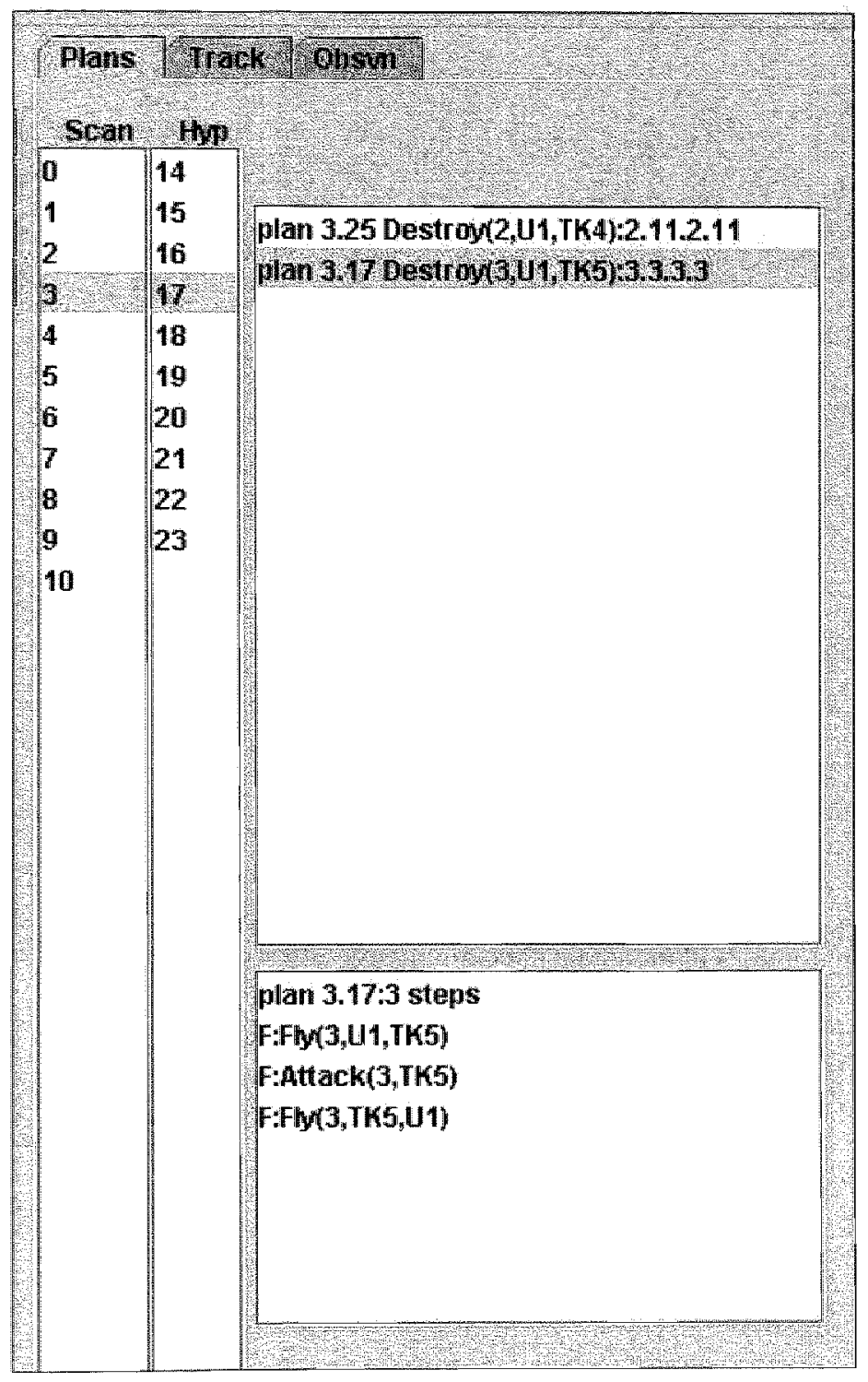

Figure 10.7: The assignment hypothesis oriented plan information display. The scan and assignment hypothesis that are selected by the user are indicated by grey lines. 


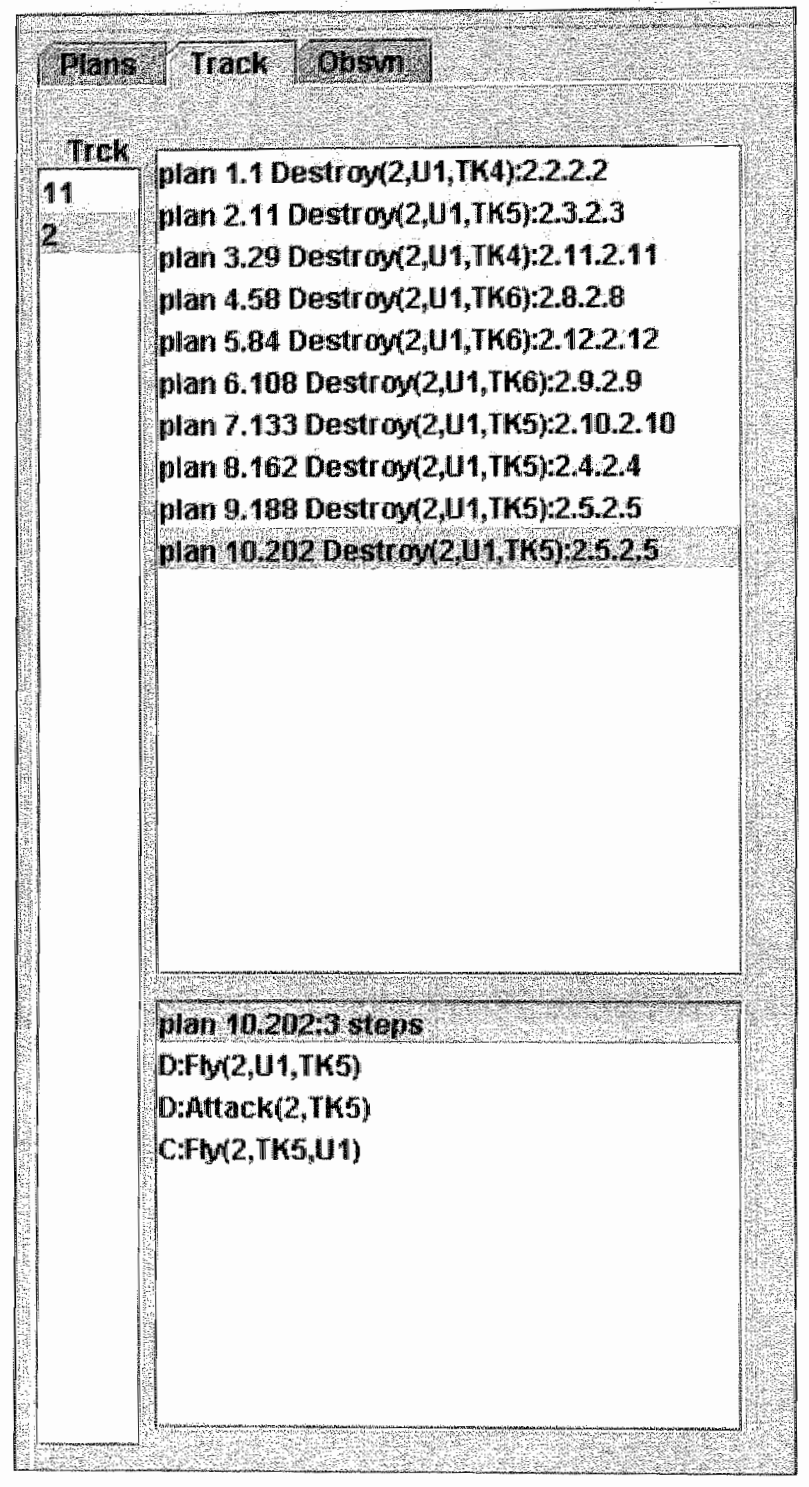

Figure 10.8: The track oriented plan information display. The evolution of the plan hypothesis for target number 2 is displayed here. 


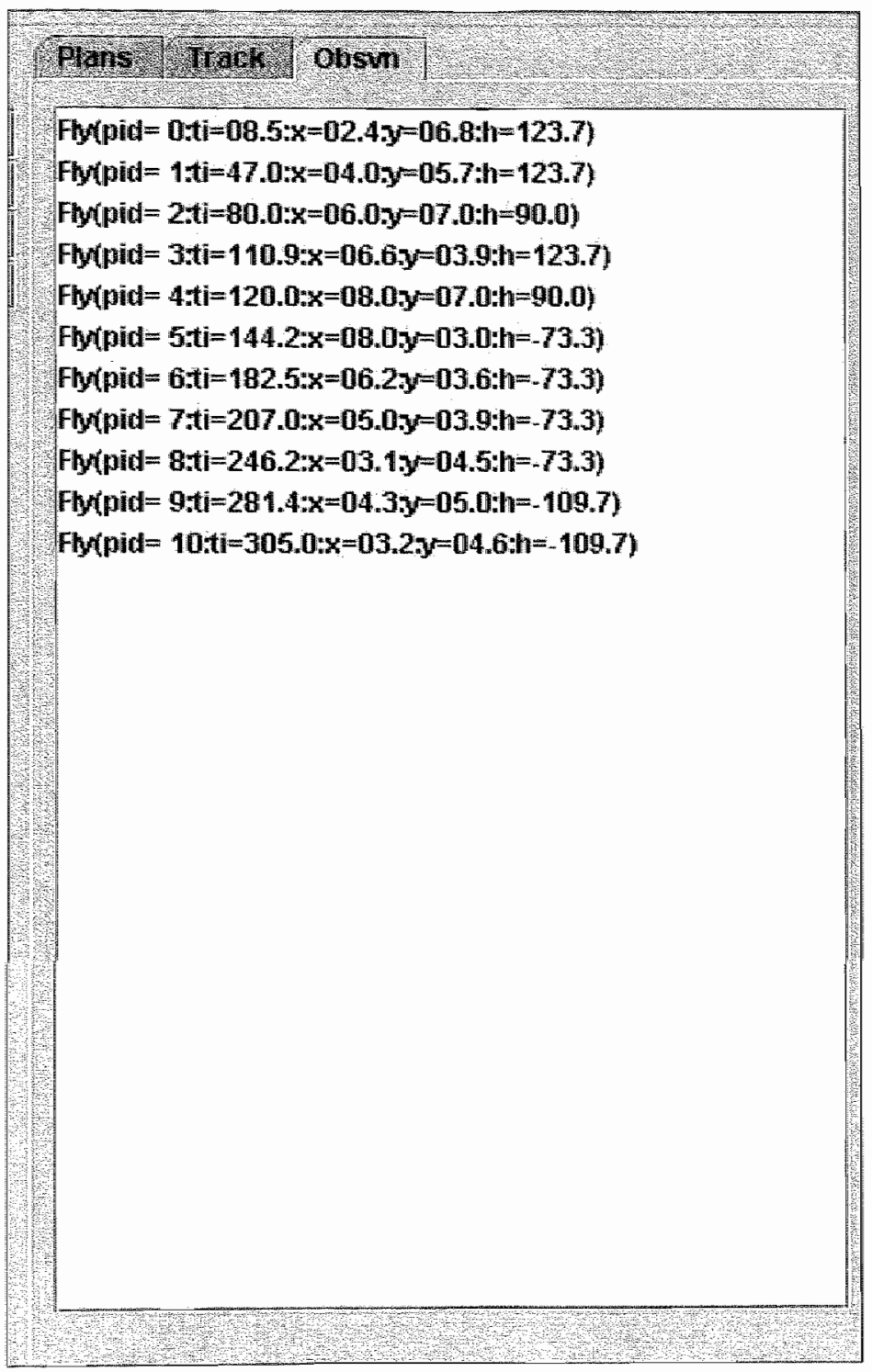

Figure 10.9: Observation display, all observations of the current scenario are displayed. In this case a number of Fly actions and their characteristics are displayed. 


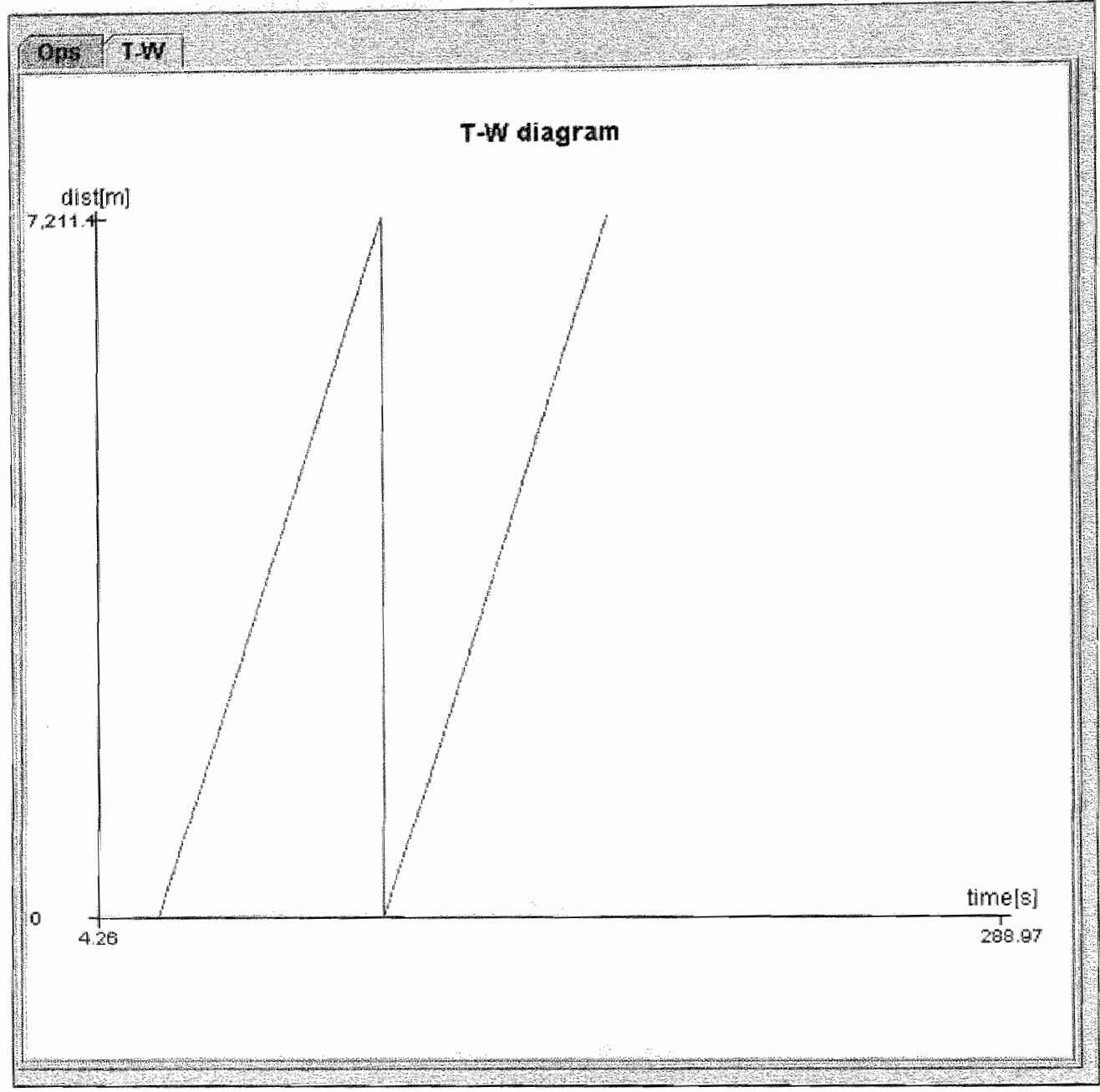

Figure 10.10: The time-distance diagram. In the diagram the $\mathrm{x}$-axis is represents the time and the vertical axis represents the clistance covered by the target on a leg of the target's trajectory. As the distance is set to zero at the start of a leg, the time-distance diagram gets a saw-tooth appearance. 


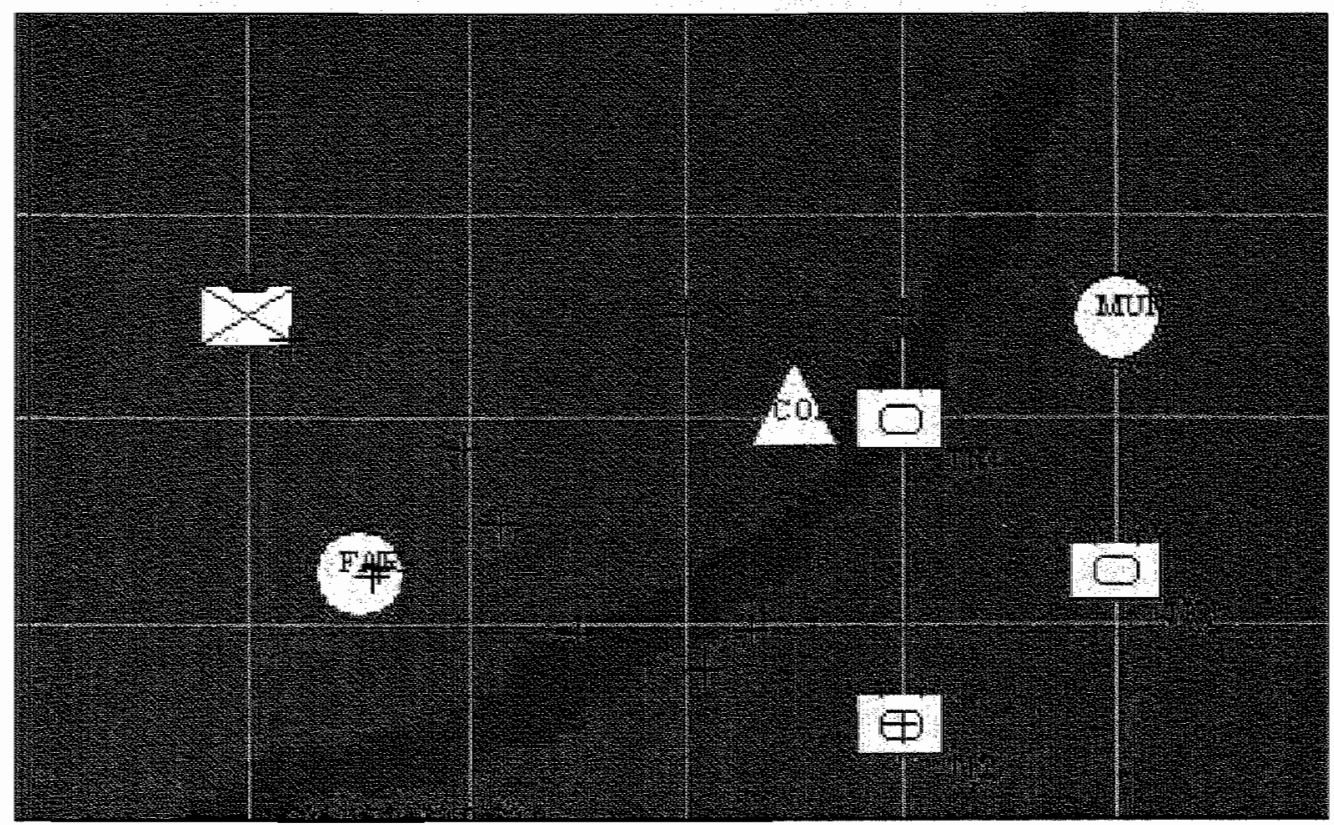

Figure 10.11: A detail of the operational display. The geography is displayed by various grey scales. A darker grey scale corresponds with a lower altitude of the geography. The various icons of the display indicate the position of military units.

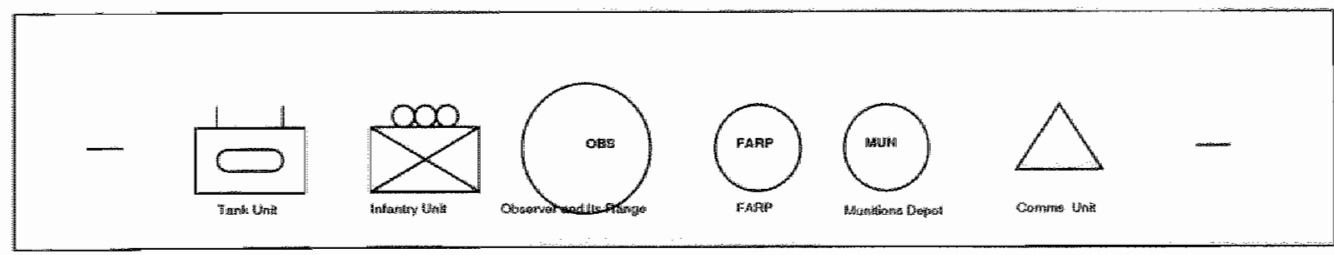

Figure 10.12: Legenda of the icons on the operational display of figure 10.11 . 


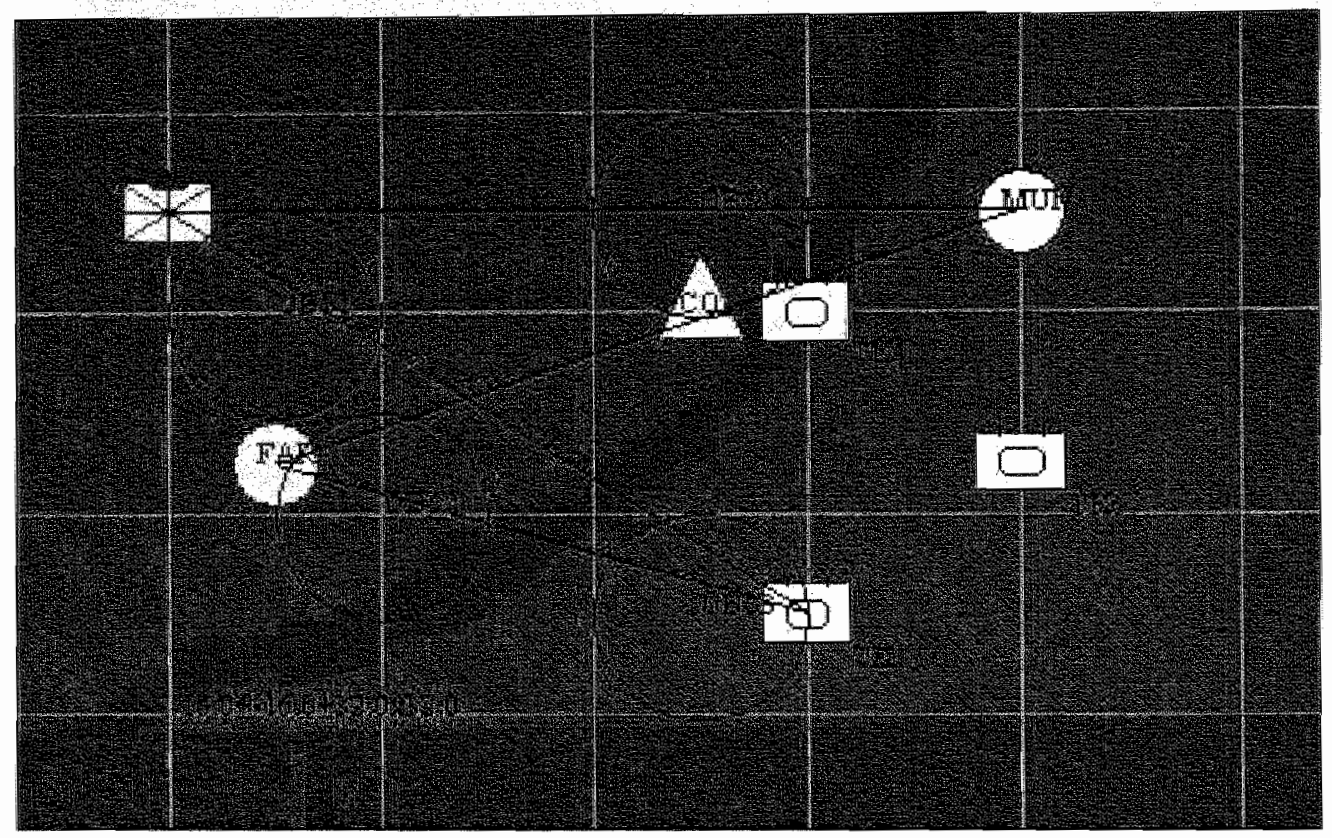

Figure 10.13: Detail of the operational display with observers (indicated by crosses and circles that represent their coverage) and the two target's possible trajectories (indicated by solid lines). 


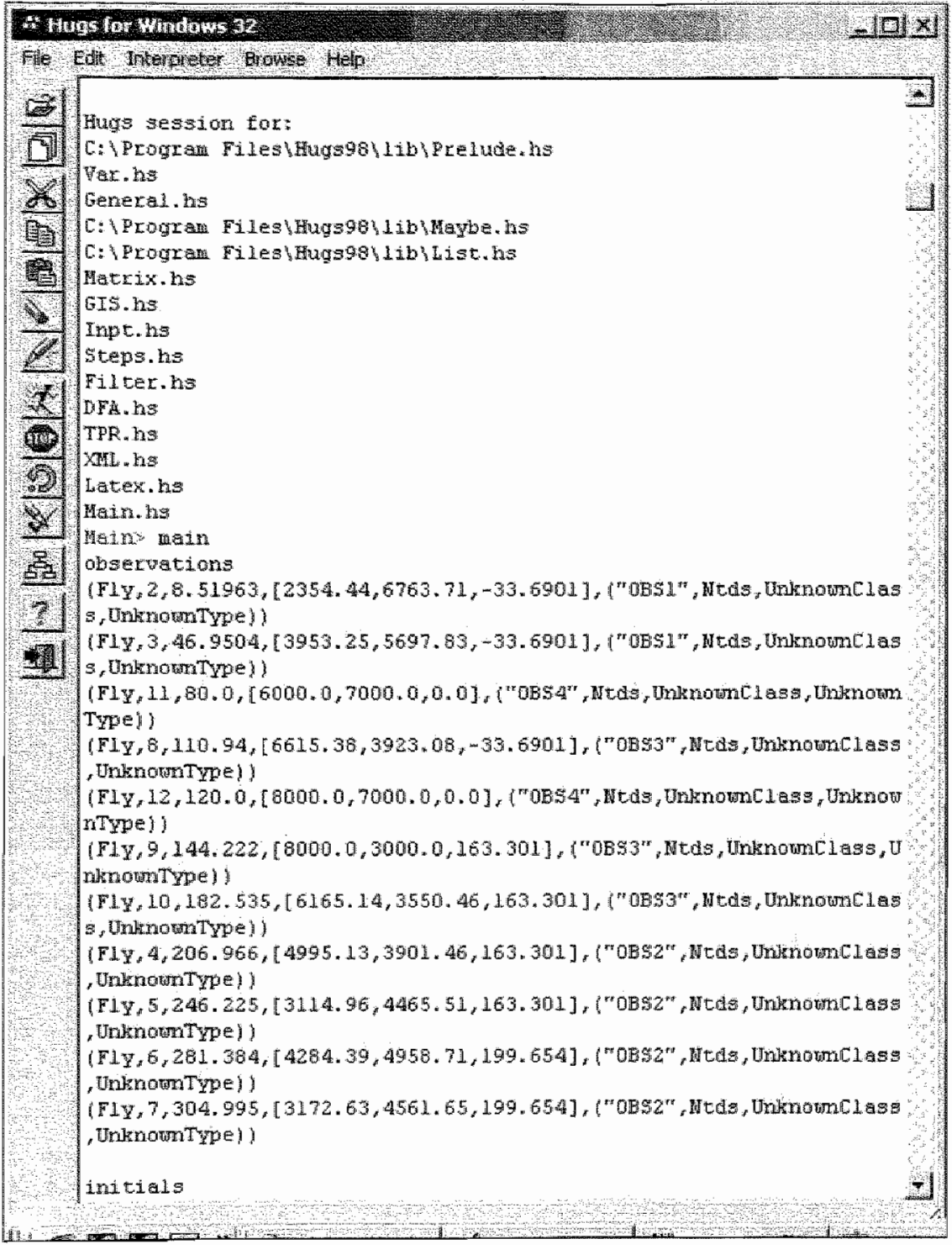

Figure 10.14: The user interface of the Hugs Haskell interpreter and running the Tactical Plan Recognition program. 


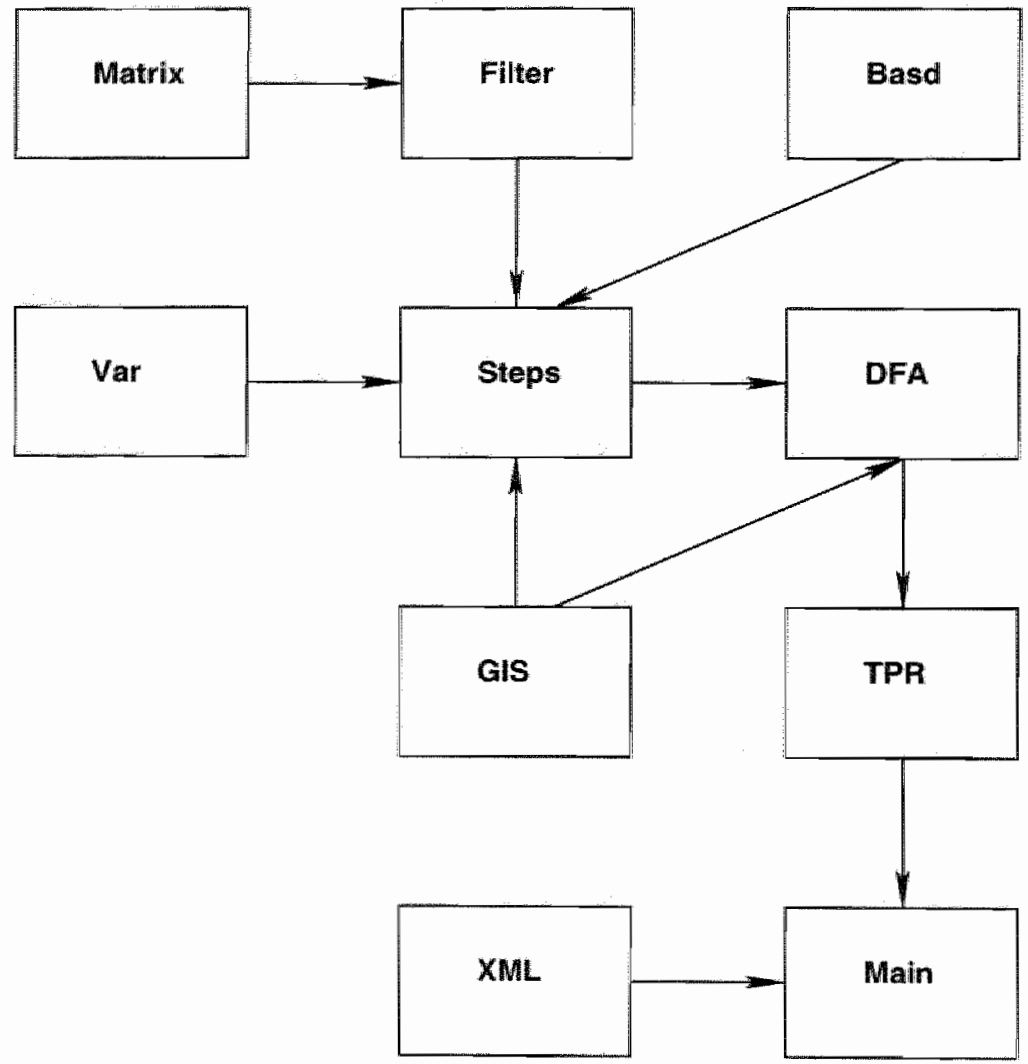

Figure 10.15: The call graph of the Haskell TPR program modules. 


\section{Chapter 11}

\section{Experiments}

In the previous chapter we discussed the design of the Demonstrator. The purpose of the Demonstrator was to show the feasibility of the approach to Tactical Plan Recognition that was introduced and discussed in the previous parts I till III. Now we are ready for observing and examining the 'real' processing of the demonstrator, for the first time.

In this chapter, two paradigmatic scenarios are defined and are input to the Demonstrator. The MHT model for tactical plan recognition, that was introduced in the previous sections, will actually solve real problems for the first time. However, the model is not complete, e.g. statistical parameters as: standard deviations of the process noise of the Kalman filters, new plan and false alarm densities that are found in Reid's formula, are not available. Only best guesses can be applied for the first run. So 'tuning' of these recognizer parameters is necessary. Also there are pruning parameters, that prevent a combinatorial explosion: for example the parameter $k$ that limits the amount of assignment hypotheses. For this one has to find a balance between calculation time and performance of the Tactical Plan Recognizer. 'Tuning a process means modifying values of various parameters for the scenario, until the performance is sufficient. The term tuning originates from tuning old fashioned radio receivers in to a specific radio station.

The first example concerns the army scenario (example 2.3). The second scenario is a new one and concerns a littoral (coastal) scenario. 


\begin{tabular}{ccccc}
$\begin{array}{c}\text { scan } \\
\text { number }\end{array}$ & action & $\begin{array}{c}\text { observation's } \\
\text { rid }\end{array}$ & observer & $\begin{array}{c}\text { time } \\
{[\mathrm{s}]}\end{array}$ \\
\hline 0 & Fly & 2 & OBS1 & 8.520 \\
1 & Fly & 3 & OBS1 & 46.950 \\
2 & Fly & 11 & OBS4 & 80.000 \\
3 & Fly & 8 & OBS3 & 110.940 \\
4 & Fly & 12 & OBS4 & 120.000 \\
5 & Fly & 9 & OBS3 & 144.222 \\
6 & Fly & 10 & OBS3 & 182.535 \\
7 & Fly & 4 & OBS2 & 209.996 \\
\hline
\end{tabular}

Table 1.1: The sequence of observations of the processing example. The scan number corresponds with the plot numbers of figure 11.3

The processing of the first example is examined in great detail. For each observation, the current set of assignment hypotheses is listed and discussed. Then the contents of the plan hypotheses of the best assignment will be presented. The effects of tuning some parameters is shown and discussed.

The second example is examined in less detail. The best assignment hypothesis after processing an observation is discussed for each of the observations. Then the performance of the Tactical Plan Recognizer is calculated. It turns out to be quite sufficient, but only after some tuning.

\subsection{The army example}

In this section the processing of the scenario, graphically displayed in figure 11.1, is examined in detail. This is done by listing the assignment hypotheses that are created by the Tactical Plan Recognizer.

The sequence of observations that are input for the plan recognizer are listed in table 11.1. The first column gives the scan number of the observation, the second column gives the observed action. The third 'rid' column gives the root id (unique identification) of the observation. Note that successive observations do not have subsequent rid's. This is a consequence of the observation generation algorithm. The next column of table 11.1 gives the observer of the observation: either OBS1, OBS2, OBS3 or OBS4. The position and coverage of these observers was displayed in figure 11.1. The final right 


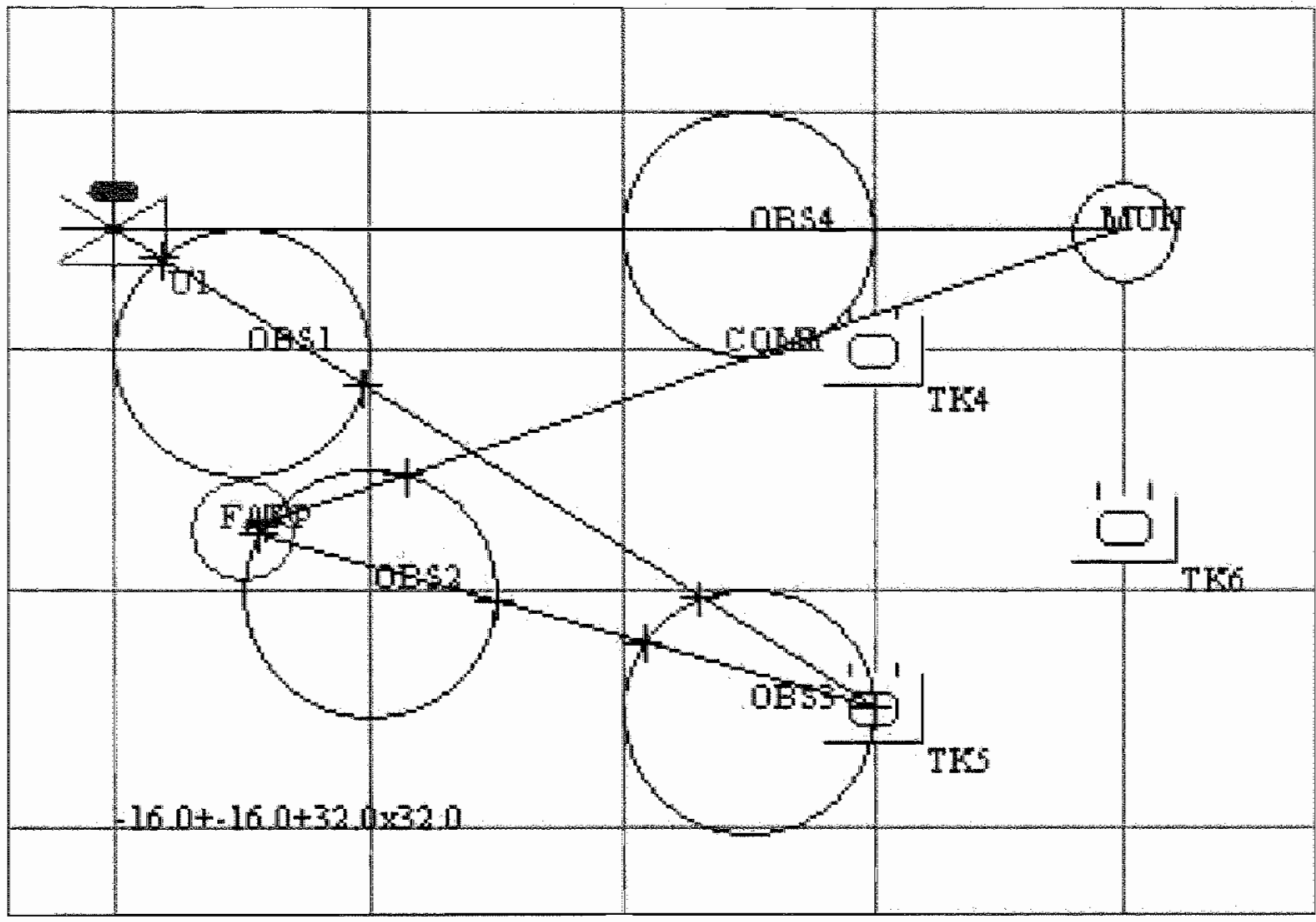

Figure 11.1: The Army scenario with two targets for examining the properties of the Tactical Plan Recognizer's processing. The icons indicate military units and the circles indicate observers and their coverage. The observer's position is in the center of the circle. The solid lines represent the trajectory of the two targets. One target flies from U1 to TK5 and back to the FARP unit. The second target flies from U1 to MUN and also back to the FARP unit

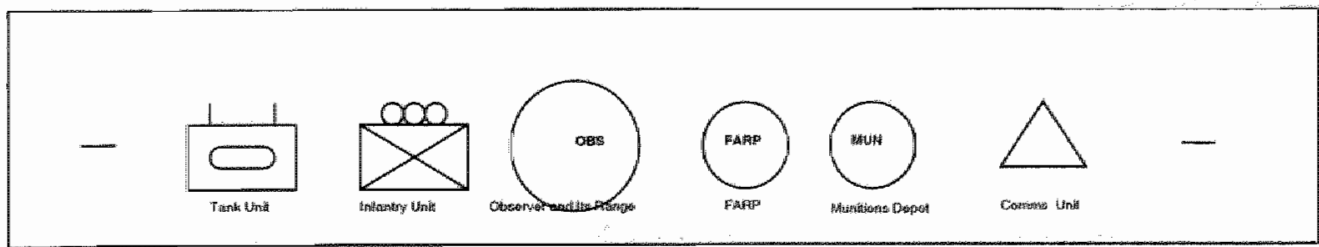

Figure 11.2: Legenda of figure 11.1. 


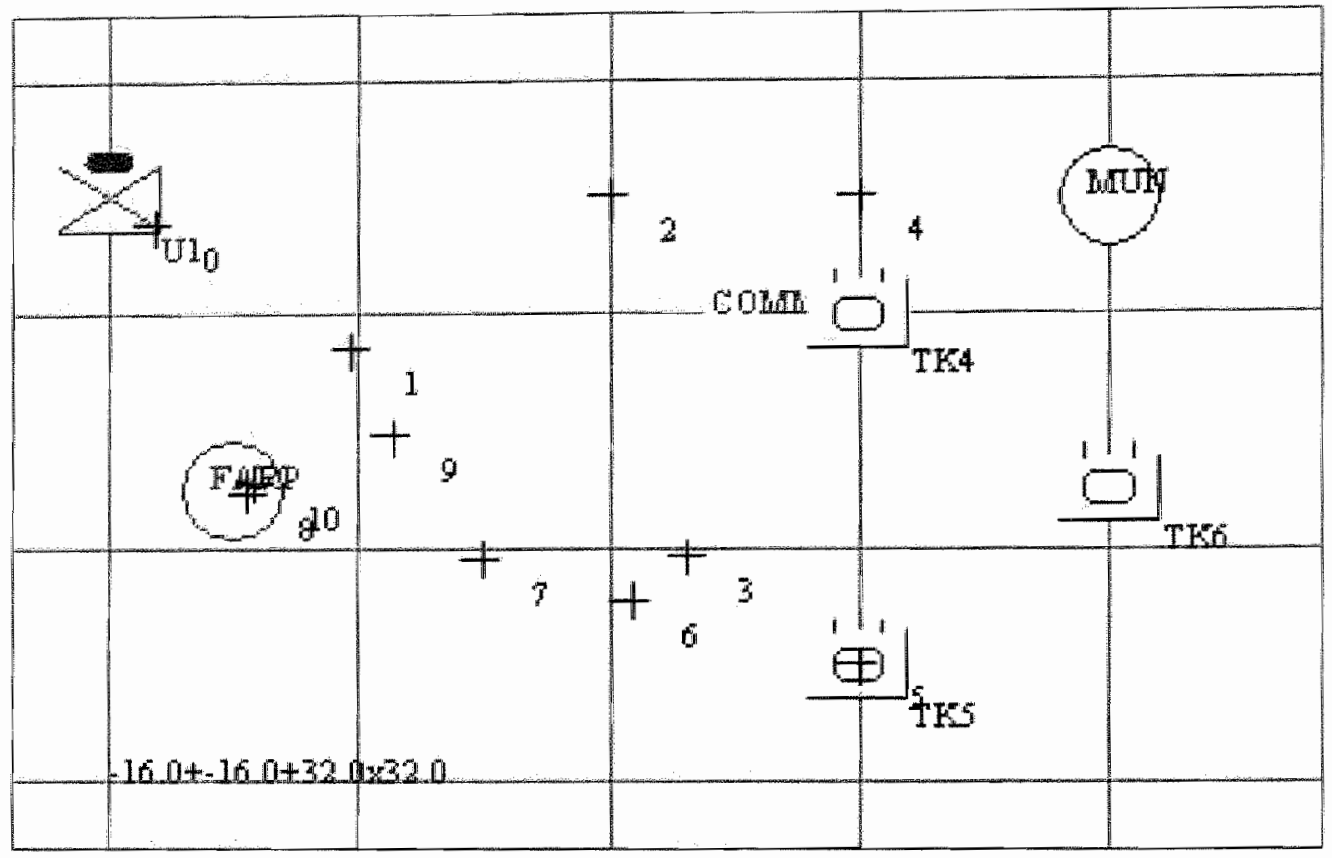

Figure 11.3: The Army scenario with the observations of the targets indicated by crosses at the observation's position. Unfortunately, the number at each observation corresponds with the scan number (see table 11.1) in which the observation occurs.

scan action observation's observer observed attacked

\begin{tabular}{clcccc} 
number & & rid & & target & target \\
\hline 0 & Fly & 2 & OBS1 & Heli 1 & TK5 \\
1 & Fly & 3 & OBS1 & Heli 1 & TK5 \\
2 & Fly & 11 & OBS4 & Heli 2 & MUN \\
3 & Fly & 8 & OBS3 & Heli 1 & TK5 \\
4 & Fly & 12 & OBS4 & Heli 2 & MUN \\
5 & Fly & 9 & OBS3 & Heli 1 & TK5 \\
6 & Fly & 10 & OBS3 & Heli 1 & TK5 \\
7 & Fly & 4 & OBS2 & Heli 1 & TK5 \\
\hline
\end{tabular}

Table 11.2: The sequence of observations and their ground truth, Heli 1 attacks unit TK5 and Heli 2 attacks unit MUN. 
column gives the time at which the target was observed.

The 'ground truth' about the observations is listed in table 11.2 (of course, the plan recognizer is unknown to this 'ground truth'). The observations are also displayed in figure 11.3. The scenario concerns two helicopters (H1 and H2) that both start from air base U1. They both execute an attack plan with the following steps:

Destroy(object, target) : Fly(base1, target), Attack(target), Fly(target, base2)

So in fact we have the following plans:

$$
\begin{aligned}
& \text { Destroy }(H 1, M U N) \text { : } \\
& \text { Fly }(H 1, U 1, M U N), \operatorname{Atack}(H 1, M U N), F l y(H 1, M U N, F A R P) \\
& \text { Destroy }(H 2, T K 5): \\
& \text { Fly }(H 2, U 1, T K 5), \text { Attack }(H 2, T K 5), F l y(H 2, T K 5, F A R P)
\end{aligned}
$$

Now examine the assignment hypotheses that are created and maintained by the plan recognizer after entering each observation.

\subsubsection{Examination of assignments}

For each scan, the number of assignment hypotheses and the number of plan hypotheses are given in a table such as table 11.3. It lists the assignment hypotheses after processing of one observation. An assignment hypothesis is displayed in one row of the table. Each row has the following columns:

- rid: the first column gives a list of the root identification numbers of the plan hypotheses. An equal root id of different tracks indicates that it concerns the same target. In fact, the root id of the plan hypothesis is the root id of the first observation of that pland hypothesis.

- nupd: the second column gives the internal numbers of the plan hypotheses, 'nupd'.

- $P(\Psi)$ : the third row gives the assignment hypothesis' probability.

The information of this paragraph are excerpts of the actual output of the Tactical Plan Recognizer Demonstrator itself, but the internal object numbers have been replaced by the object's names. In the case that an observation is thought to refer to a false alarm by the plan recognizer this is identified by a 'FA' in the 'upd' column of the assignment and the absence of a root id. 


\begin{tabular}{ccc} 
rid & nupd & $P(\Psi)$ \\
\hline 2 & 1 & 0.322581 \\
2 & 2 & 0.322581 \\
2 & 3 & 0.322581 \\
& FA & 0.032258 \\
\hline
\end{tabular}

Table 11.3: Assignment hypotheses after the first observation. The list of plan hypotheses is $[1,2,3]$.

\subsubsection{Scan 0, Observation 2, Observer OBS1}

This concerns observation 2 of Heli 1 and the measurement time is 8.51963 [s]. Now first consider the assignment hypotheses that are generated after processing this first observation.

Assignment hypotheses. There are four assignment hypotheses and three plan hypotheses. Note that the sum of the assignment hypothesis probabilities is one. The assignments of the first observation are listed in the table 11.3.

When the first observation is entered into the system, three new plan hypotheses are created for it. One plan attack plan hypotheses is created for each of the enemy tank units (TK4, TK5, TK6). The target is not expected to attack the munitions depot (MUN), because its heading is more south than an attack on the munitions depot requires. In table 11.3, the assignment hypotheses are displayed.

Note that each different attack plan has a different internal track number ('mupd' column), but that the root id ('rid' column) is the same. This indicates that it concerns one and the same object. The probabilities are equal. At this stage, the plan recognizer cannot decide on the best assignment hypothesis. This is understandable, as there is only one observation.

Best plan hypotheses. The single plans of the best three assignment hypotheses, concern the attack, by a target numbered 2 , starting from base U1 and attacking target 4,5 or 6 (TK4, TK5 or TK6). Apparently the plan recagnizer cannot recognize that the attack concerns the munitions depot. The actual output of the plan recognizer is: 


\begin{tabular}{cccc}
$\begin{array}{c}\text { track } \\
\text { rumber }\end{array}$ & goal & $\begin{array}{c}\text { begin } \\
\text { time }\end{array}$ & $\begin{array}{c}\text { current } \\
\text { time }\end{array}$ \\
\hline$(1,1,2,2)$ & (Destroy, $[2,1,4])$ & 4.25982 & 8.51963 \\
\hline
\end{tabular}

This output is not very readable, the plan hypothesis number is quite complicated: $(1,1,2,2)$ and the list of Destroy: $[2,1,4]$ contains references to objects in the Army scenario. The first element of the track number is the number of the parent plan hypothesis, the second number is the id of the plan hypothesis itself, the third number is the root id (rid) of the first supporting plot of the plan hypothesis and the final number is the root id of the last supporting observation of the plan hypothesis. The output of the plan recognizer with the proper substitutions $(2 / \mathrm{H} 2,1 / \mathrm{U} 1,4 / \mathrm{TK} 4)$ and additional columns for the rid and upd of a plan bypothesis is:

\begin{tabular}{cccccc}
$\begin{array}{c}\text { track } \\
\text { number }\end{array}$ & nupd & rid & goal & $\begin{array}{c}\text { begin } \\
\text { time }\end{array}$ & $\begin{array}{c}\text { current } \\
\text { time }\end{array}$ \\
\cline { 1 - 6 }$(1,1,2,2)$ & 1 & 2 & (Destroy, $[$ H2,U1,TK4]) & 4.25982 & 8.51963 \\
\hline
\end{tabular}

Note that $\mathrm{H} 2$ is an internal representation for a unique Heli target, but has no relation to the ground truth 'Heli 1' or 'Heli 2' identification. The actual plan concerns the following steps:

1. (Fly, 60.8276, 6082.76, [H2,U1,TK4], Future), meaning: target 2 flies from unit U1 to unit TK4. The leg takes 61 seconds and the length of the leg is 6082 meters. The plan recognizer has not set the execution state of the action yet, it still is planned in the future.

2. (Attack, 0.0, 0.0, [H2,TK4], Future),

3. (Fly, 60.8276,6082.76, [H2,TK4,U1], Future): here the target returns to the base it started from. At this stage of the processing it is not possible to recognize that the helicopter will return to the FARP unit.

\subsubsection{Scan 1, Observation 3, Observer OBS1}

This concerns observation 3 of Heli 1 , observed at a time of $46.9504[\mathrm{~s}]$. The assignment hypotheses after processing this observation are discussed below. 
Assignment hypotheses. After processing the observation by the plan recognition system, there are 10 assignment hypotheses and 9 plan hypotheses. In fact, the parameter $k$ that represents the maximal number of hypotheses that is retained after each scan is $10: k=10$ and pruning has been invoked here. The assignment hypotheses are listed below. Note that the sum of the probabilities is again one.

\begin{tabular}{ccc} 
rid & nupd & $P(\Psi)$ \\
\hline 2 & 7 & 0.3588010 \\
2 & 8 & 0.2403710 \\
2 & 6 & 0.1497270 \\
3,2 & 10,1 & 0.0358716 \\
3,2 & 11,1 & 0.0358716 \\
3,2 & 12,1 & 0.0358716 \\
3,2 & 10,2 & 0.0358716 \\
3,2 & 11,2 & 0.0358716 \\
3,2 & 12,2 & 0.0358716 \\
3,2 & 10,3 & 0.0358716 \\
\hline
\end{tabular}

The list of plan hypotheses is: $[1,2,3,10,11,12,6,7,8]$. This set contains the numbers that occur in the 'nupd' column. The order of the numbers is quite random and is the order in which they are stored internally in the program.

When the second observation (nr. 3) of the previous target is entered into the system, new internal correlated plans are created. These result in new plan hypotheses 7 till 12 (nupd). It is recognized by the processing that plans $6,7,8$ concern the same target as in the previous scan because of their identical root ids (rid). The assignment probabilities now differ significantly. Apparently the recognizer can now decide on a best hypotheses. This because there now have been two observations, that clearly show a flight in the direction of one the tank units.

From the fourth assignment hypothesis onward, the assignment contains two plan hypotheses. So in these cases, the two observations are expected to originate from two different targets by the tactical plan recognizer. Of course, from the ground truth, we know that observations 2 and 3 originate from Heli 1 , so this is not the case. Luckily, the probability of these untrue assignment hypotheses is low. 
Best plan hypotheses. The best hypothesis its only plan hypothesis is displayed below. The goal has now changed from an attack on unit TK4 to an attack on unit TK5:

\begin{tabular}{cccccc}
$\begin{array}{c}\text { plan hyp } \\
\text { number }\end{array}$ & mupd & rid & goal & $\begin{array}{c}\text { begin } \\
\text { time }\end{array}$ & $\begin{array}{c}\text { current } \\
\text { time }\end{array}$ \\
\hline$(7,2,2,3)$ & 7 & 3 & (Destroy, [H2, U1,TK5]) & 4.25982 & 46.9504 \\
\hline
\end{tabular}

The steps of the plan are as follows:

1. (Fly, 131.137, 7211.1, [H2,U1,TK5], Current), now the plan recognizer has adapted the estimated speed of the target and also the execution state of this first leg has been set to 'currently being executed'. The observation time of observation 11, is within the begin and end time of this leg from U1 to TK5 and the fly action is labelled 'Current'

2. (Attack, 0.0,0.0, [H2,TK5], Future),

3. (Fly, 131.137, 7211.1, [H2,TK5,U1], Future)

\subsubsection{Scan 3, Observation 11, Observer OBS4}

The observation 11 concerns Heli 2 and its measurement time is $80.0[\mathrm{~s}]$. The assignment hypotheses after processing this observation are given below.

Assignment hypotheses. After processing the new observation, there are 10 assignment hypotheses and 13 plan hypotheses. The assignment hypotheses are listed below.

\begin{tabular}{ccc} 
rid & nupd & $P(\Psi)$ \\
\hline 2 & 21 & 0.3380940 \\
2 & 23 & 0.3206300 \\
2,3 & 17,10 & 0.0674725 \\
2,3 & 17,11 & 0.0674725 \\
2,3 & 17,12 & 0.0674725 \\
2 & 22 & 0.0635255 \\
3,2 & 18,1 & 0.0196079 \\
3,2 & 18,2 & 0.0196079 \\
3,2 & 18,3 & 0.0196079 \\
11,2 & 25,7 & 0.016509 \\
\hline
\end{tabular}


The list of plan hypotheses is: $[1,2,3,10,11,12,7,25,17,18,21,22,23]$.

The third observation (nr. 11) is the first observation of the second target, H1, that attacks the munitions depot (MUN). When it enters the plan recognizer, plan hypotheses 17 till 25 are created. The best assignment hypothesis still contains only one plan hypothesis, with the same external plan hypothesis number as in the previous scan. For the new observation, only one plan hypothesis is created internally. Because of the heading of the target, an attack on another unit than the munitions depot is unlikely. The new plan is only part of an assignment with a low probability, because the new target density parameter, that is used in Reid's formula, equation 5.2, of the MHT, decreases the probability of the tenth assignment.

Best plan hypotheses. The best hypothesis its only plan hypothesis is displayed below. Thought the observation concerns another target, it is incorrectly correlated with the plan hypothesis of target number 2. The target of the plan remains TK5. Because this observation has a higher y-position, nearer to the $y$-position of the MUN object, and the combined direction of flight is more towards unit TK5:

\begin{tabular}{cccccc}
$\begin{array}{c}\text { plan hyp } \\
\text { number }\end{array}$ & nupd & rid & goal & $\begin{array}{c}\text { begin } \\
\text { time }\end{array}$ & $\begin{array}{c}\text { current } \\
\text { time }\end{array}$ \\
\hline$(21,6,2,11)$ & 21 & 2 & (Destroy, [H2,U1,TK5]) & 4.25982 & 80.0 \\
\hline
\end{tabular}

The steps of the plan are as follows:

1. (Fly, 115.177, 6082.76, [H2,U1, TK5], Current), now the plan recognizer has adapted the estimated speed of the target again.

2. (Attack, 0.0,0.0, [H2,TK5], Future),

3. (Fly, 161.194,8381.53, [H2,TK5,U1], Future)

\subsubsection{Scan 3, Observation 8, Observer OBS3}

This concerns observation 8 of Heli 1 and the observation time is 110.94[s]. The assignment hypotheses are listed in the following paragraph.

Assignment hypotheses. There are 10 assignment hypotheses and 12 plan hypotheses. The assignment hypotheses are listed below. 


\begin{tabular}{ccc} 
rid & nupd & $P(\Psi)$ \\
\hline 2 & 46 & 0.3200570 \\
3,2 & 39,17 & 0.2338470 \\
2 & 45 & 0.2211510 \\
2 & 44 & 0.0771201 \\
2,11 & 41,25 & 0.0548499 \\
8,2 & 48,21 & 0.0189874 \\
8,2 & 49,21 & 0.0189874 \\
8,2 & 50,21 & 0.0189874 \\
8,2 & 48,23 & 0.0180066 \\
8,2 & 49,23 & 0.0180066 \\
\hline
\end{tabular}

The list of plan hypotheses is $[25,17,21,23,48,49,50,39,41,44,45,46]$

In this scan, observation nr. 8 is entered into the recognizer. This is a continuation of plan hypothesis with rid $=2$ and its plan hypothesis is found in the best assignment.

Best plan hypotheses. The best assignment's hypothesis its only plan hypothesis is displayed below. The observation concerns the helicopter of plan hypothesis number 2 ( rid $=2$ ) and is correctly correlated to it. However, due to the previous correlation with the other helicopter, it is now thought to concern an attack on unit TK6, that is in-between TK5 and the munitions depot.

\begin{tabular}{cccccc}
$\begin{array}{c}\text { plan hyp } \\
\text { number }\end{array}$ & nupd & rid & goal & $\begin{array}{c}\text { begin } \\
\text { time }\end{array}$ & $\begin{array}{c}\text { current } \\
\text { time }\end{array}$ \\
\hline$(21,6,2,11)$ & 21 & 2 & (Destroy,[H2,U1,TK6]) & 4.25982 & 110.94 \\
\hline
\end{tabular}

The steps of the plan are as follows:

1. (Fly, 161.68, 8381.53, [H2,U1, TK6] , Current), now the plan recognizer has adapted the estimated speed of the target again.

2. (Attack, 0.0,0.0, [H2,TK6], Future),

3. (Fly, 161.194, 8381.53, [H2,TK6,U1], Future) 


\subsubsection{Scan 4, Observation 12, Observer OBS4}

This concerns the observation 12 of Heli 2 at $120.0[\mathrm{~s}]$. The assignment hypotheses after processing the observation are listed below.

Assignment hypotheses. There are 10 assignment hypotheses and 14 plan hypotheses. The assignment hypotheses are listed below.

\begin{tabular}{ccc} 
rid & nupd & $P(\Psi)$ \\
\cline { 1 - 3 } 2 & 73 & 0.3394170 \\
2 & 71 & 0.2559670 \\
2,8 & 64,48 & 0.0627655 \\
2,8 & 64,49 & 0.0627655 \\
2,8 & 64,50 & 0.0627655 \\
8,2 & 66,21 & 0.0620171 \\
8,2 & 66,23 & 0.0588137 \\
11,2 & 63,41 & 0.0399727 \\
12,2 & 75,46 & 0.0277578 \\
12,2 & 76,46 & 0.0277578 \\
\hline
\end{tabular}

The list of plan hypotheses is $[21,23,48,49,50,41,46,75,76,63,64,66,71,73]$.

Now observation $\mathrm{nr} .12$ of the second target becomes leads to two plan hypotheses. They are found in the lowest assignments and still no plan hypothesis of the second target is outputted.

Best plan hypotheses. The best plan is similar to that of the previous observation. In the best assignment hypothesis there is still only one target recognized. The previously best plam hypothesis is only updated with the new observation.

\subsubsection{Scan 5, Observation 9, Observer OBS3}

This concerns observation 9 of Heli 1 at $144.222[\mathrm{~s}]$. The assignment hypotheses are listed below.

Assignment hypotheses. Now there are 10 assignment hypotheses and 13 plan hypotheses. The assignment hypotheses are listed below. 


\begin{tabular}{ccc} 
rid & nupd & $P(\Psi)$ \\
\hline 2 & 96 & 0.3110890 \\
2,11 & 93,63 & 0.2962560 \\
8,2 & 91,64 & 0.1183580 \\
2,8 & 90,66 & 0.0510524 \\
9,2 & 98,73 & 0.0410332 \\
9,2 & 99,73 & 0.0410332 \\
9,2 & 100,73 & 0.0410332 \\
8,2 & 92,64 & 0.0382558 \\
9,2 & 98,71 & 0.0309448 \\
9,2 & 99,71 & 0.0309448 \\
\hline
\end{tabular}

The list of plan hypotheses is $[63,64,66,71,73,98,99,100,90,91,92,93,96]$.

Now a new observation (nr. 9) of the first target is input. Now we see that still only one plan hypothesis is in the first assignment, but the true assignment with plans 2 and 11 gets to the second place.

Best plan hypotheses. The best plan is similar to that of the previous observation. In the best assignment hypothesis there is still only one target recognized. The previously best plan hypothesis is only updated with the new observation.

\subsubsection{Scan 6, Observation 10, Observer OBS3}

This concerns observation 10 of Hel 1 at $182.535[\mathrm{~s}]$. After processing this observation, the assignment hypothesis are:

Assignment hypotheses. Now there are 10 assignment hypotheses and 13 plan hypotheses. The assignment hypotheses are listed below: 


\begin{tabular}{ccc} 
rid & nupd & $P(\Psi)$ \\
\hline 2,11 & 116,63 & 0.8186390 \\
9,2 & 113,73 & 0.0355269 \\
9,2 & 113,71 & 0.0267922 \\
11,2 & 109,93 & 0.019771 \\
10,2 & 119,96 & 0.0167597 \\
10,2 & 120,96 & 0.0167597 \\
10,2 & 121,96 & 0.0167597 \\
10,2 & 122,96 & 0.0167597 \\
9,2 & 114,73 & 0.0162711 \\
$10,2,11$ & $119,93,63$ & 0.0159606 \\
\hline
\end{tabular}

The list of plan hypotheses is $[63,71,73,93,96,119,120,121,122,109,113,114,116]$.

Best plan hypotheses. Finally, when the observation nr. 9 of the first target is input for the plan recognizer, the assignment, with two plan hypotheses of two separate targets gets the highest probability. At this point the plan recognizer can output two plan hypotheses. One for each of the two targets.

\begin{tabular}{cccccc}
$\begin{array}{c}\text { plan hyp } \\
\text { number }\end{array}$ & upd & rid goal & begin & current \\
time & time \\
\hline$(116,93,2,10)$ & 116 & 2 & (Destroy, [H2,U1, TK5]) & 4.25982 & 182.535 \\
$(63,25,11,12)$ & 63 & 11 & (Destroy, [H11,U1, TK4]) & 40.0 & 120.0 \\
\hline
\end{tabular}

The steps of the first plan are as follows:

1. (Fly, 161.138, 7211.1,[H2,U1,TK5],Done), now the plan recognizer has recognized that the first step of the plan has been executed. Therefore its state is done.

2. (Attack, 0.0, 0.0, [H2,TK6], Done),

3. (Fly, 149.033, 7211.1, [H2,TK5,H1], Current), the plan recognizer has set the state of the last leg to currently being executed.

The steps of the second plan are as follows: 
1. (Fly, 60.8276, 6082.76,[H11, U1, TK4], Done)

2. (Attack, $0.0,0.0,[2,6]$, Done),

3. (Fly, 709.377, 6082.76, [H2,TK6,U1], Current)

\subsubsection{Scan 7, Observation 4, Observer OBS2}

This concerns observation 4 of Heli 1 at 206.966[s]. The assignment hypotheses after processing the new observation are listed below.

Assignment hypotheses. There are 10 assignment hypotheses and 15 plan hypotheses. The assignment hypotheses are listed below:

\begin{tabular}{ccc} 
rid & nupd & $P(\Psi)$ \\
\hline 2,11 & 143,63 & 0.83857 \\
$4,2,11$ & $145,116,63$ & 0.0207883 \\
$4,2,11$ & $146,116,63$ & 0.0207883 \\
$4,2,11$ & $147,116,63$ & 0.0207883 \\
$4,2,11$ & $148,116,63$ & 0.0207883 \\
2,11 & 136,109 & 0.0192884 \\
10,2 & 139,96 & 0.0170632 \\
$2,10,11$ & $136,119,63$ & 0.0155711 \\
9,2 & 142,73 & 0.0147389 \\
11,2 & 133,116 & 0.0116153 \\
\hline
\end{tabular}

The list of plan hypotheses is $[63,73,96,119,109,116,145,146,147$, $148,133,136,139,142,143]$

When observation nr. 4 is entered into the system, the correct assignment hypothesis with plans 2 and 11 remains to have the highest probability.

Best plan hypotheses. The plan hypotheses are updated versions of that from the previous scan. 


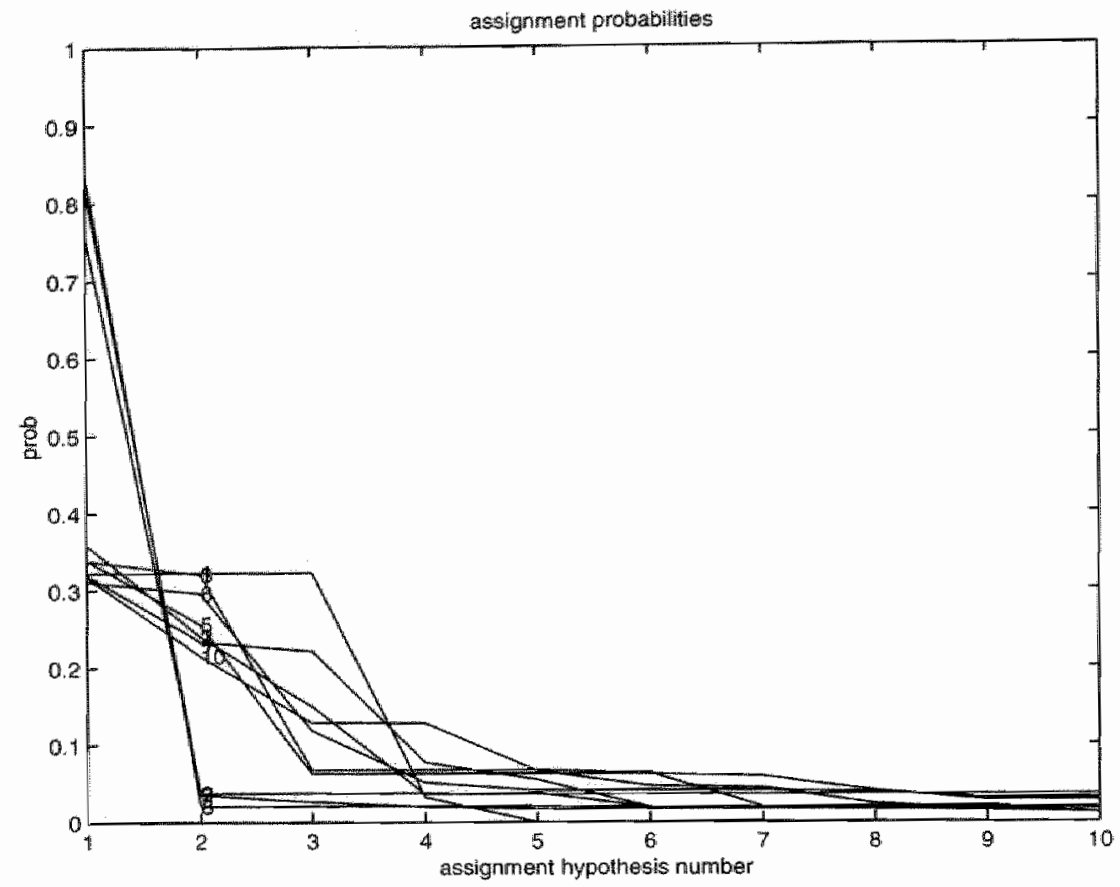

Figure 11.4: Graph of the assignment probabilities for different scans.

\subsubsection{Conclusion}

So now we have examined the processing of a sequence of observations. Some shortcomings have been noted. The plan recognizer does create plans for different targets but not for different return bases. Hence all plans return to the airbase $U 1$ in stead of the actual return base FARP. The plan recognizer could only recognize in a too late stage, when the flights were returning, that the observations concerned two targets. Before that point of time, it correlated all observations. Consequently, the goal target of the plans varied between TK4, TK6 and TK5.

\subsubsection{Examination of probabilities}

In figure 11.4 the assignment probabilities are displayed for all hypotheses. Each scan corresponds with one graph and the scan number is label for the graph. The figure shows that the plan recognizer can decide upon a particular 


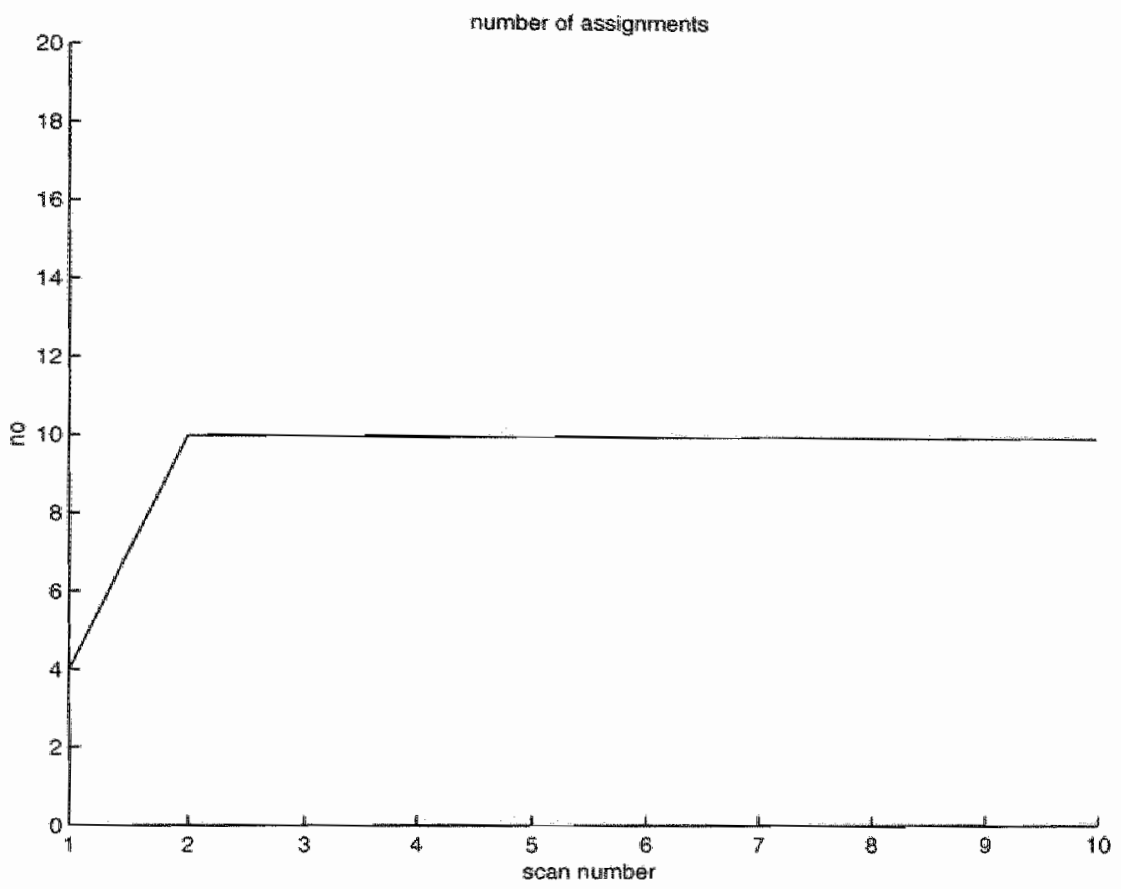

Figure 11.5: Graph of the number of assignment hypotheses as a function of the scan number. 
assignment for all scans, except for the first scan. In the first few scans, the variation in the assignment probabilities does vary about 0.1 . Only in the last few scans, the highest probability exceeds the others with a value of about 0.6 . So only then the plan recognizer can really make a clear choice.

The number of assignment hypotheses increases very fast from an initial value of 4 till the maximum of 10 . So in this example the 10-best assignment hypotheses are retained by the plan recognizer. The number of assignment hypotheses as a function of the time are displayed in figure 11.5.

\subsubsection{Performance of the Tactical Plan Recognizer}

The recognized plans of the run of the scenario are listed in table 11.4. For this scenario one can calculate the performance numbers of table 11.5. A definition of the performance numbers is given below:

1. separate target: this is the number of observations that a target is recognized as a separate target divided by the total number of observations of the target. Ideally, this performance number should be $100 \%$.

2. first correct plan: this is the number of observations after which the plan of a target is recognized correctly divided by the total number of observations of the target. Ideally, this measure is $0 \%$ indicating immediate recognition of the plan.

3. number of correct plans: this is a quotient of the total number of observations of the target, during which the plan of the target is recognized correctly divided by the total number of observations of the target. Ideally this measure is a $100 \%$.

4. track continuity: this is the percentage of observations of a target during which it is recognized correctly as one target. This measure does not take in account, whether the plan of the target has be recognized correctly. Ideally, this measure is $100 \%$.

The performance measures are expressed with percentages of the observations. For example, the first correct plan for the helicopter $\mathrm{H} 1$ was recognized at the first observation, so the number of observations it took was zero. The total number of observations that are entered into the system are eight. So the percentage for first correct plan is $0 / 8=0 \%$. The first observation concerning helicopter $\mathrm{H} 2$ is the third one, so remain 6 observations for helicopter 


\begin{tabular}{|c|c|c|c|c|c|}
\hline $\begin{array}{c}\text { scan } \\
\text { number }\end{array}$ & $\begin{array}{c}\text { observation } \\
\text { number }\end{array}$ & observer & $\begin{array}{c}\text { observed } \\
\text { target }\end{array}$ & $\begin{array}{c}\text { attacked } \\
\text { target }\end{array}$ & $\begin{array}{c}\text { recognized } \\
\text { plans }\end{array}$ \\
\hline 1 & 2 & $\overline{\mathrm{OBS} 1}$ & $\mathrm{H1}$ & TK5 & Destroy(H2, U1,TK4) \\
\hline 2 & 3 & OBS1 & H1 & TK5 & $\operatorname{Destroy}(\mathrm{H} 2, \mathrm{U} 1, \mathrm{TK} 5)$ \\
\hline 3 & 11 & OBS4 & $\mathrm{H} 2$ & MUN & Destroy (H2, U1,TK4) \\
\hline 4 & 8 & OBS3 & $\mathrm{H} 1$ & TK5 & Destroy $(\mathrm{H} 2, \mathrm{U} 1, \mathrm{TK} 6)$ \\
\hline 5 & 12 & OBS4 & $\mathrm{H}_{2}$ & MUN & $\operatorname{Destroy}\left(\mathrm{H}_{2}, \mathrm{U} 1, \mathrm{TK} 6\right)$ \\
\hline 6 & 9 & OBS3 & $\mathrm{H} 1$ & TK5 & $\operatorname{Destroy}\left(\mathrm{H}_{2}, \mathrm{C} 1, \mathrm{TK} 6\right)$ \\
\hline 7 & 10 & OBS3 & $\mathrm{H} 1$ & TK5 & $\begin{array}{l}\text { Destroy }(\mathrm{H} 2, \mathrm{U} 1, \mathrm{TK} 5) \\
\text { Destroy }(\mathrm{H} 11, \mathrm{U} 1, \mathrm{TK} 4)\end{array}$ \\
\hline 8 & 4 & OBS2 & H1 & TK5 & $\begin{array}{l}\text { Destroy }(\mathrm{H} 2, \mathrm{U} 1, \mathrm{TK} 5), \\
\text { Destroy }(\mathrm{H} 11, \mathrm{U} 1, \mathrm{TK} 4)\end{array}$ \\
\hline
\end{tabular}

Table 11.4: The sequence of observations and accompanying best plan hypotheses of the army example, before tuning.

\begin{tabular}{l|llll} 
helicopter & $\begin{array}{l}\text { separate } \\
\text { target }\end{array}$ & $\begin{array}{l}\text { first correct } \\
\text { plan }\end{array}$ & $\begin{array}{l}\text { number correct } \\
\text { plan }\end{array}$ & $\begin{array}{l}\text { track } \\
\text { continuity }\end{array}$ \\
\hline $\mathrm{H} 1$ & $0 / 8=0 \%$ & $2 / 8=25 \%$ & $3 / 8=37.5 \%$ & $8 / 8=100 \%$ \\
$\mathrm{H} 2$ & $2 / 6=33 \%$ & $0 / 6=0 \%$ & $0 / 6=0 \%$ & $2 / 6=33 \%$ \\
\hline
\end{tabular}

Table 11.5: Performance of army scenario.

H2. Note that no time is used in the performance measures. The observations are very sparse and a time based performance measure would even be more dependent on the amount of sensors and observers in the area of the scenario. The incorrect egress leg (towards U1 in stead of towards the FARP) is not considered in the performance.

\subsubsection{Tuning}

Clearly the performance is not very good yet and further tuning of the application is required. The first issue is that it takes too long before two separate targets are distinguished in the best assignment hypothesis. 
First tuning action. To improve on target separation consider the following observations:

- With the current set of parameters, often good correlations between observations and plans of different helicopters occur. This results in combining plans hypotheses of different targets. To improve on this, the number of correlations is restricted.

- The new plan density or new target density, $\beta_{N T}$, of Reid's formula (equation 5.2) was set at 0.001 targets/scan and was equal to that of the false target density $\beta_{F A}$. It is now increased to 0.01 targets/scan. As a consequence of this change, new plans have a better change to compete with correlations (some of which were incorrect), represented by the correlation likelihood, $g_{x y}$, in Reid's formula.

With this new set of parameters, the first separate track for the second helicopter is now created only one scan after its first observation.

The best hypotheses, after this tuning action, are displayed in table 11.6. The new performances are displayed in table 11.7. The resolution in two targets has improved. But still the target of the second helicopter is not recognized properly and the track continuity of the second target is poor.

Second tuning action. To improve the correct recognition of the plan of the second helicopter, we now focus on the correlation and the influence of the heading on the correlation. The standard deviation of the heading is decreased from 20 degrees to 10 degrees. Now observations on the same leg will correlated better than observations from other legs, with different headings. The output of the plan recognizer is given in table 11.8 and the performance is given in table 11.9. The performance for the second helicopter has improved significantly: the number of correct plans have increased from $0 \%$ to $100 \%$. However there is a slight decrease in the track continuity of the first target: from $100 \%$ to $75 \%$.

\subsection{The littoral scenario}

In this scenario, an amphibious task force is preparing a landing operation under protection of a frigate. Several Landing and Transport Ships (LTS) 


\begin{tabular}{|c|c|c|c|c|c|}
\hline $\begin{array}{c}\text { scan } \\
\text { number }\end{array}$ & $\begin{array}{c}\text { observation } \\
\text { number }\end{array}$ & observer & $\begin{array}{c}\text { observed } \\
\text { target }\end{array}$ & $\begin{array}{c}\text { attacked } \\
\text { target }\end{array}$ & $\begin{array}{c}\text { recognized } \\
\text { plans }\end{array}$ \\
\hline 1 & 2 & OBS1 & $\mathrm{HI}$ & $\mathrm{TK} 5$ & Destroy $(\mathrm{H} 2, \mathrm{U} 1, \mathrm{TK} 4)$ \\
\hline 2 & 3 & OBS1 & $\mathrm{H} 1$ & TK5 & Destroy $(\mathrm{H} 2, \mathrm{U} 1, \mathrm{TK} 5)$ \\
\hline 3 & 11 & OBS4 & $\mathrm{H}_{2}$ & MUN & Destroy $(\mathrm{H} 2, \mathrm{U} 1, \mathrm{TK} 6)$ \\
\hline 4 & 8 & OBS3 & $\mathrm{H} 1$ & TK5 & $\begin{array}{l}\text { Destroy }(\mathrm{H} 2, \mathrm{U} 1, \mathrm{TK} 4) \text {, } \\
\text { Destroy }(\mathrm{H} 3, \mathrm{U} 1, \mathrm{TK} 5)\end{array}$ \\
\hline 5 & 12 & OBS4 & $\mathrm{H}_{2}$ & MUN & $\begin{array}{l}\text { Destroy }(\mathrm{H} 2, \mathrm{U} 1, \mathrm{TK} 6) \\
\operatorname{Destroy}(\mathrm{H} 8, \mathrm{U} 1, \mathrm{MUN})\end{array}$ \\
\hline 6 & 9 & OBS3 & $\mathrm{H} 1$ & TK5 & $\begin{array}{c}\text { Destroy (H2,U1, TK4), } \\
\text { Destroy (H3,U1, TK5 }) \\
\text { Destroy (H12,U1, TK5) }\end{array}$ \\
\hline 7 & 10 & OBS3 & H1 & TK5 & $\begin{array}{l}\text { Destroy (H2, U1, TK5), } \\
\text { Destroy (H11, U1,TK4) }\end{array}$ \\
\hline 8 & 4 & OBS2 & $\mathrm{H} 1$ & TK5 & $\begin{array}{l}\text { Destroy (H2,U1, TK5), } \\
\text { Destroy (H11, U1,TK4) }\end{array}$ \\
\hline
\end{tabular}

Table 11.6: The sequence of observations and accompanying best plan hypotheses of the army example, after the first tuning action.

\begin{tabular}{l|llll} 
helicopter & $\begin{array}{l}\text { separate } \\
\text { target }\end{array}$ & $\begin{array}{l}\text { first correct } \\
\text { plan }\end{array}$ & $\begin{array}{l}\text { number correct } \\
\text { plan }\end{array}$ & $\begin{array}{l}\text { track } \\
\text { continuity }\end{array}$ \\
\hline H1 & $0 / 8=0 \%$ & $2 / 8=25 \%$ & $3 / 8=37.5 \%$ & $8 / 8=100 \%$ \\
H2 & $1 / 6=17 \%$ & $2 / 6=30 \%$ & $0 / 6=0 \%$ & $4 / 6=67 \%$ \\
\hline
\end{tabular}

Table 11.7: Performance of the army scenario after the first tuning action. 


\begin{tabular}{|c|c|c|c|c|c|}
\hline $\begin{array}{l}\text { scan } \\
\text { number }\end{array}$ & $\begin{array}{l}\text { observation } \\
\text { number }\end{array}$ & observer & $\begin{array}{c}\text { observed } \\
\text { object }\end{array}$ & $\begin{array}{l}\text { attacked } \\
\text { target }\end{array}$ & $\begin{array}{l}\text { recognized } \\
\text { plans }\end{array}$ \\
\hline 1 & 2 & OBS1 & $\mathrm{H} 1$ & TK5 & Destroy $(\mathrm{H} 2, \mathrm{U} 1, \mathrm{TK} 4)$ \\
\hline 2 & 3 & OBS1 & $\mathrm{HI}$ & TK5 & Destroy(H3,U1,TK5) \\
\hline 3 & 11 & OBS4 & $\mathrm{H} 2$ & MUN & Destroy $(\mathrm{H} 2, \mathrm{U1}, \mathrm{TK} 4)$ \\
\hline 4 & 8 & OBS3 & $\mathrm{H} 1$ & TK5 & $\begin{array}{l}\text { Destroy }(\mathrm{H} 3, \mathrm{U} 1, \mathrm{TK} 4) \\
\text { Destroy }(\mathrm{H} 8, \mathrm{U} 1, \mathrm{MUN})\end{array}$ \\
\hline 5 & 12 & OBS4 & $\mathrm{H} 2$ & MUN & $\begin{array}{l}\operatorname{Destroy}(\mathrm{H} 2, \mathrm{U} 1, \mathrm{TK} 5) \text {, } \\
\operatorname{Destroy}(\mathrm{H} 11, \mathrm{U} 1, \mathrm{MUN})\end{array}$ \\
\hline 6 & 9 & OBS3 & H1. & TK5 & $\begin{array}{l}\text { Destroy }(\mathrm{H} 2, \mathrm{U} 1, \mathrm{TK} 5), \\
\text { Destroy }(\mathrm{H} 11, \mathrm{U} 1, \mathrm{MUN})\end{array}$ \\
\hline 7 & 10 & OBS3 & $\mathrm{H} 1$ & TK5 & $\begin{array}{c}\text { Destroy }(\mathrm{H} 2, \mathrm{U} 1, \mathrm{TK} 5) \\
\text { Destroy }(\mathrm{H} 11, \mathrm{U} 1, \mathrm{MUN})\end{array}$ \\
\hline 8 & 4 & OBS2 & $\mathrm{H} 1$ & TK5 & $\begin{array}{c}\text { Destroy }(\mathrm{H} 2, \mathrm{U} 1, \mathrm{TK} 5) \\
\operatorname{Destroy}(\mathrm{H} 11, \mathrm{U} 1, \mathrm{MUN})\end{array}$ \\
\hline
\end{tabular}

Table 11.8: The sequence of observations and accompanying plan hypotheses of the army example, after the final (second) tuning.

\begin{tabular}{l|llll} 
helicopter & $\begin{array}{l}\text { separate } \\
\text { target }\end{array}$ & $\begin{array}{l}\text { first correct } \\
\text { plan }\end{array}$ & $\begin{array}{l}\text { number correct } \\
\text { plan }\end{array}$ & $\begin{array}{l}\text { track } \\
\text { continuity }\end{array}$ \\
\hline H1 & $0 / 8=0 \%$ & $1 / 8=13 \%$ & $5 / 8=62.5 \%$ & $6 / 8=75 \%$ \\
H2 & $1 / 6=17 \%$ & $2 / 6=30 \%$ & $6 / 6=100 \%$ & $4 / 6=67 \%$ \\
\hline
\end{tabular}

Table 11.9: Performance of the army scenario after the final tuning action. 
have to make trips to the coast and back to the ship over a distance of 5 Nautical Miles (NM).

The opposed forces of the country in crises send two Fast Patrol Boats (FPB) equipped with anti-ship missiles: they left their harbor at $40 \mathrm{NM}$ from the operation area. The first FPB (FPB1) sails along the coast, while the second FPB (FPB2) passes several coastal islands and then sails toward the frigate. Until 14 NM from the frigate, FPB1 is partially masked by a group of islands. In the vicinity of the islands, the speed of the FPBs is $10[\mathrm{~m} / \mathrm{s}]$ and they are guided by a flying drone (also called an Unaccompanied Arial Vehicle, UAV). After passing the islands, FPB1 and the frigate are in sight of each other. The second FPB (FPB2) sails further from the coast. The mission of FPB1 is to attack the frigate with a missile and the mission of the FPB2 is to jam ${ }^{1}$ the radar equipment of the frigate in order to support the mission of FPB1.

The geography of the scenario is given in figure 11.6. The altitudes are displayed with by their altitude that is expressed by a lighter grey scale. The sea is colored black. The observers are posted on the high grounds near the coast and are indicated by coverage circles. The initial position of the FPBs is at the top left of the map. The planned trajectories are indicated by two solid lines. These start at the top left initial position and end near the center of the map, where the frigate to be attacked is located. The trajectory of the FPBs and the observations (indicated by crosses) are also given in figure 11.8.

For this scenario, two plan templates are available, one for an attack on an enemy object and one that concerns a jamming operation. They consist of an ingress step, a destroy resp. jamming action and the egress. The jamming operation concerns only the frigate, so a dinghy ${ }^{2}$ or the marine infantry unit ashore are not targets for a jamming operation.

\subsubsection{The best assignment hypotheses before tuning}

In this subsection we will examine the processing of the Demonstrator. As in the previous example, some parameter values are initially only guessed and have to be tuned to this type of scenario. If the performance is unsatisfactory, a tuning action will follow. The processing is not presented in the same amount of detail of the Army example. But now only the plan hypotheses of the best

\footnotetext{
The purpose of radlar jamming is to, electronically, disturb the proper functioning of a radar.

${ }^{2} \mathrm{~A}$ dinghy is an inflatable small boat.
} 


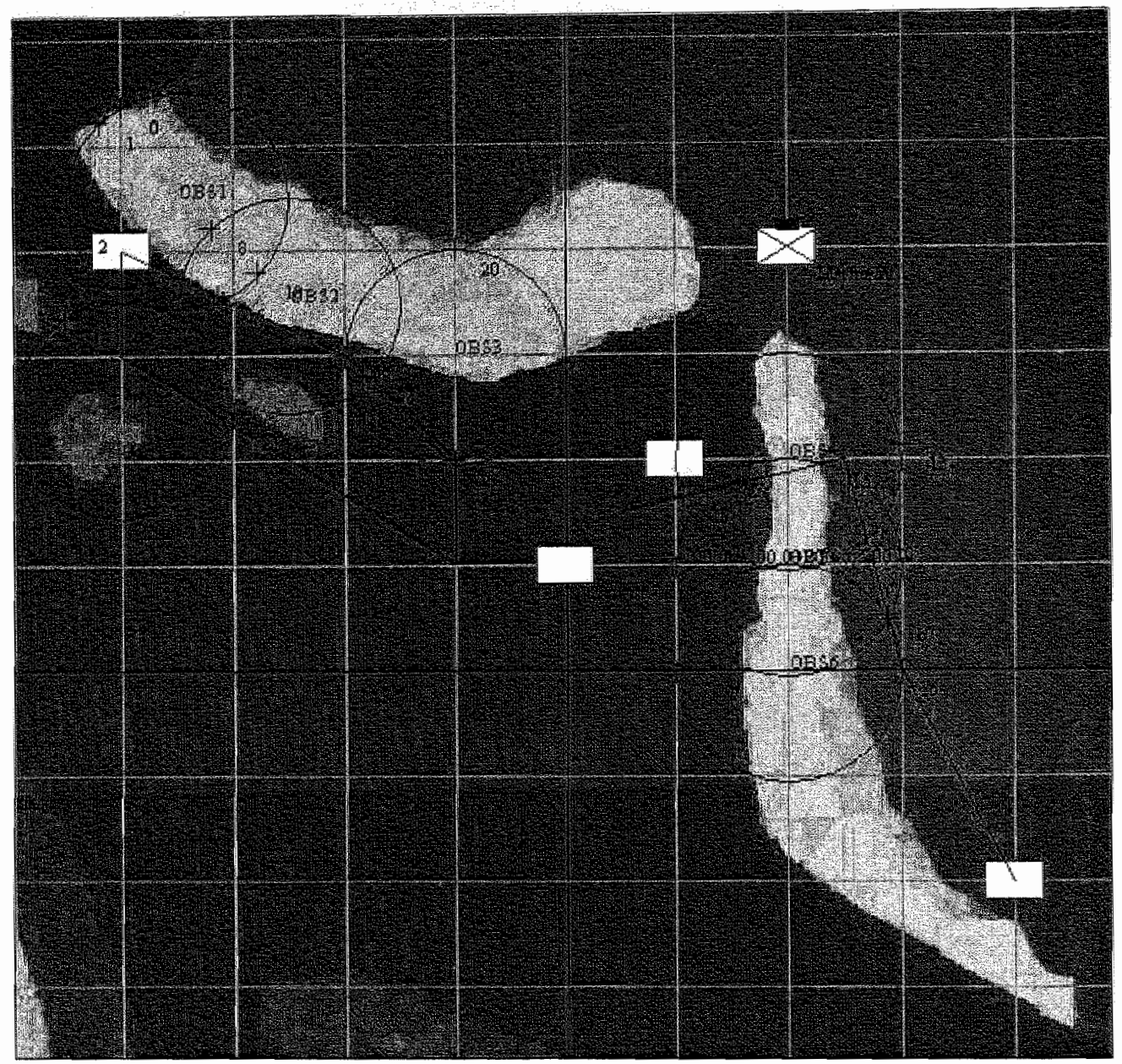

Figure 11.6: A map of the littoral scenario. The darkest areas represent the sea, the lighter areas concern land. The altitude of the ground is indicated by the grey scale. 


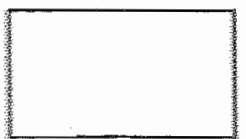

Unitship

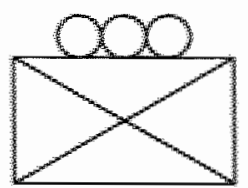

Marine infantry Unit
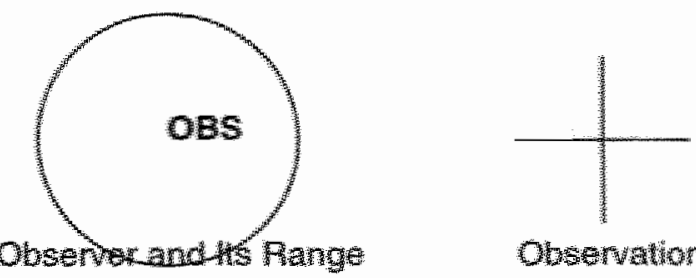

Obserwation

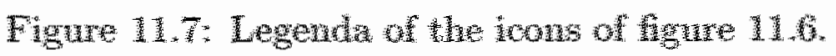

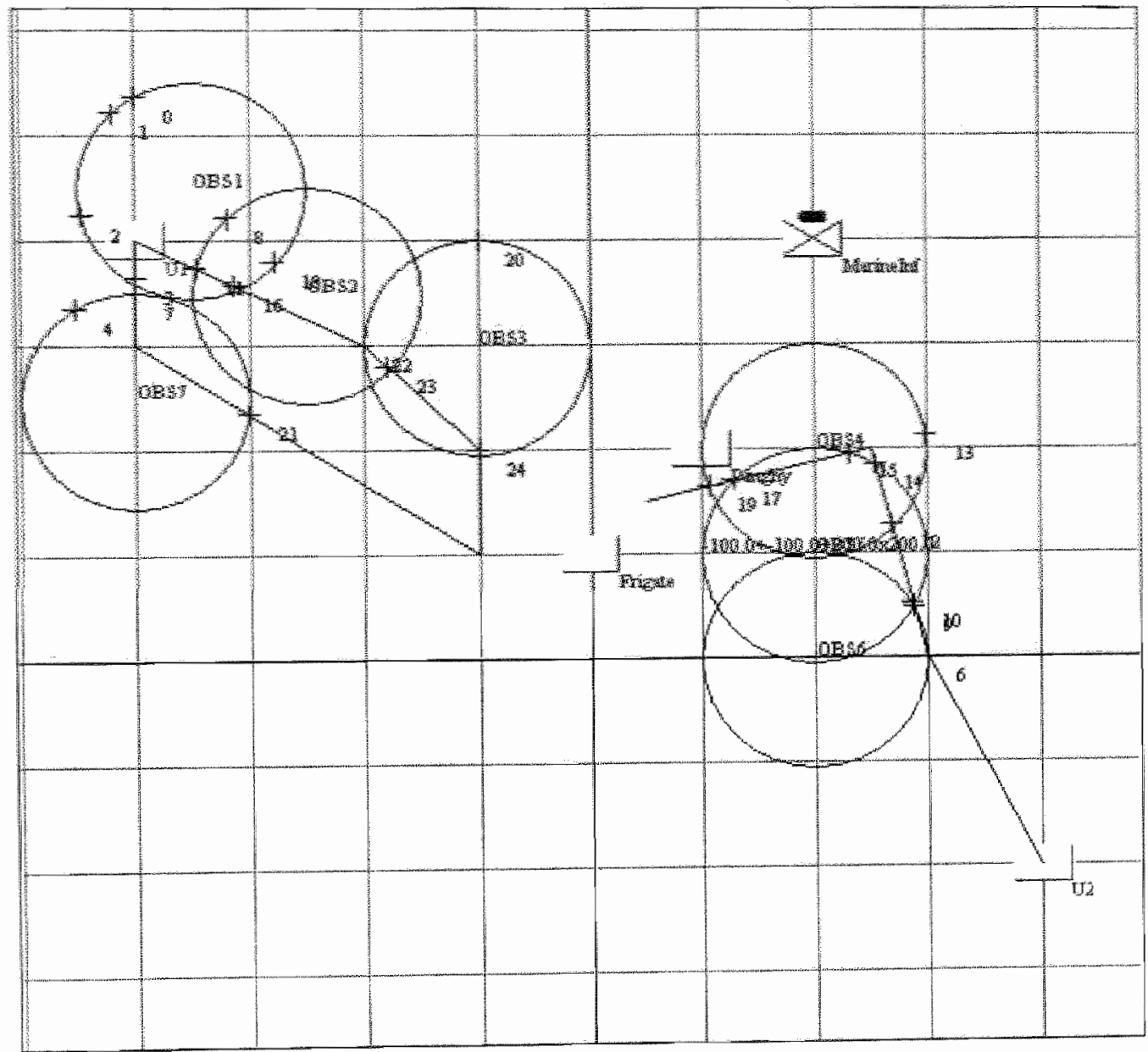

Figure 11.8: The FPB trajectories, observers and their observations in the littoral scenario. 
assignment hypotheses, after processing an observation, are listed in table 11.12 .

The first observation (scan $=1$ ) of the first Fast Patrol Boat FPB1 results in a plan hypothesis of the attack of the Dinghy: Destroy(FPB1, U1, Dinghy). The real target is the frigate, but the plan recognizer cannot discern an attack on the dinghy from an attack on the frigate.

The second observation (scan $=2$ ) concerns the second. Fast Patrol Boat FPB2. It results in a separate plan hypothesis: Destroy(FPB2,Dinghy, U1). This is, again, an incorrect plan hypothesis of an attack on the frigate.

The third observation (scan $=3$ ) concerns FPB2 again. However, it does not correlate optimally with the previous plan hypothesis of FPB2. A new plan hypothesis: Destroy(FPB3,Dinghy,U2), is created. Following observations only result in even more new plans for separate Fast Patrol Boats.

The performance is quite bad, no generated plan hypothesis is correct and often there are more than two plan hypotheses. So there are more plan hypotheses than targets. In all cases, the dinghy is the preferred attack object. So, clearly, the initial guess for tuning parameters fails. To improve the performance, shortcomings of the best plan hypotheses are identified and a related tuning parameter changed. The tuning process is discussed in the next subsection.

\subsubsection{Tuning}

First of all, there are too many plan hypotheses. Though there are only two FPBs, the Demonstrator's 'Tactical Plan Recognition system outputs three plan hypotheses. The superfluous plan hypothesis is an 'old' one, that has not been updated with new observations for some time and is of no great value anymore. So the first thing to do is to remove such 'old' plan hypotheses. For this a time-out of 1500 seconds is introduced. If a plan hypothesis has not been updated for a period of time, longer than the time-out, the plan hypothesis is removed from the system.

Also, the plan recognizer does not yet choose for the jamming action for FPB2. The cause of this is that the correlator does not check whether the target object of the jamming plan hypothesis is a frigate ${ }^{3}$. So this check is added to the correlator function. This has quite a good effect, after the

\footnotetext{
${ }^{3}$ This is not really a tuning problem, but lacking of essential knowledge in the Tactical Plan Recognizer.
} 


\begin{tabular}{|c|c|c|c|}
\hline $\begin{array}{c}\text { scan } \\
\text { number }\end{array}$ & $\begin{array}{c}\text { observed } \\
\text { target }\end{array}$ & $\begin{array}{l}\text { attack } \\
\text { target }\end{array}$ & $\begin{array}{c}\begin{array}{c}\text { recognized } \\
\text { plans }\end{array} \\
\end{array}$ \\
\hline 1 & FPB1 & Frig & Destroy(FPB1, U1, Dinghy) \\
\hline 2 & EPB2 & Frig & $\begin{array}{l}\text { Destroy(FPB1,U1, Dinghy), } \\
\text { Destroy(FPB2,U1, Dinghy) }\end{array}$ \\
\hline 3 & FPB2 & Frig & $\begin{array}{l}\text { Destroy(FPB1, U1,Dinghy), } \\
\text { Destroy(FPB2,U1,Dinghy) } \\
\text { Destroy(FPB3,U1,Dinghy) }\end{array}$ \\
\hline 4 & FPB2 & Frig & $\begin{array}{l}\text { Destroy(FPB1,U1, Dinghy), } \\
\text { Destroy(FPB2,U1,Dinghy) } \\
\text { Destroy(FPB3,U1,Dinghy) }\end{array}$ \\
\hline 5 & FPB1 & Frig & $\begin{array}{l}\text { Destroy(FPB1,U1, Dinghy), } \\
\text { Destroy(FPB2,U1, Dinghy) } \\
\text { Destroy(FPB3,U1,Dinghy) }\end{array}$ \\
\hline 6 & FPBI & Frig & $\begin{array}{l}\text { Destroy(FPB1, U1, Dinghy), } \\
\text { Destroy(FPB2,U1,Dinghy) } \\
\text { Destroy(FPB3,U1,Dinghy) }\end{array}$ \\
\hline 7 & FPB1 & Frig & $\begin{array}{l}\text { Destroy(FPB1,U1, Dinghy), } \\
\text { Destroy(FPB2,U1,Dinghy) } \\
\text { Destroy(FPB3,U1,Dinghy) }\end{array}$ \\
\hline 8 & FPB1 & Frig & $\begin{array}{l}\text { Destroy(FPB1,U1, Dinghy), } \\
\text { Destroy(FPB2,U1, Dinghy) } \\
\text { Destroy(FPB3,U1, Dinghy), } \\
\text { Destroy(FPB16,U1, Dinghy) }\end{array}$ \\
\hline
\end{tabular}

Table 11.10: The best assignment hypothesis of the littoral example before tuning. 


\begin{tabular}{|l|c||c|}
\hline \multicolumn{2}{|c||}{ parameter } & value \\
\hline \hline old plan hypothesis time-out & $T_{\text {old }}$ & $1500[\mathrm{~s}]$ \\
\hline number hypothesis retained & $\mathrm{k}$ & 10 \\
\hline detection probability & $P_{D}$ & 0.99 \\
\hline number of new plans/scan & $N_{N P}$ & 0.0001 \\
\hline number of false plans/scan & $N_{F P}$ & 0.001 \\
\hline process noise of the distance & $\sigma_{d}$ & $10.000[\mathrm{~m}]$ \\
\hline process noise of the speed & $\sigma_{v}$ & $10.0[\mathrm{~m} / \mathrm{s}]$ \\
\hline
\end{tabular}

Table 11.11: Tuning parameters of the Demonstrator and their values.

first observation of FPB2, an attack on the frigate is assumed. As soon as a jamming observation enters the system, the attack of the frigate plan is replaced by a jamming plan hypothesis.

An overview of the most important tuning parameter is given in table 11.1.1. For this set of tuning parameters, the best assignment hypotheses are given in table 11.12. The performance is quite good, only the track continuity for the FPB1 target is not perfect. The first correct plan for FPB2 only is available at the observation of scan 7 , where (for the first time) a jamming action is observed. So only then, the correct jamming plan can be outputted. Consequently, the first completely correct plan for FPB2 is only available after $50 \%$ of the observations. Here we are limited by the available observations and the tuning process is finished.

This finishes the experiments with our Tactical Plan Recognizer. Unfortunately the range is only limited to two scenarios. Nevertheless some experience has been gained. In the next chapter an evaluation of various aspects of the research is given. 


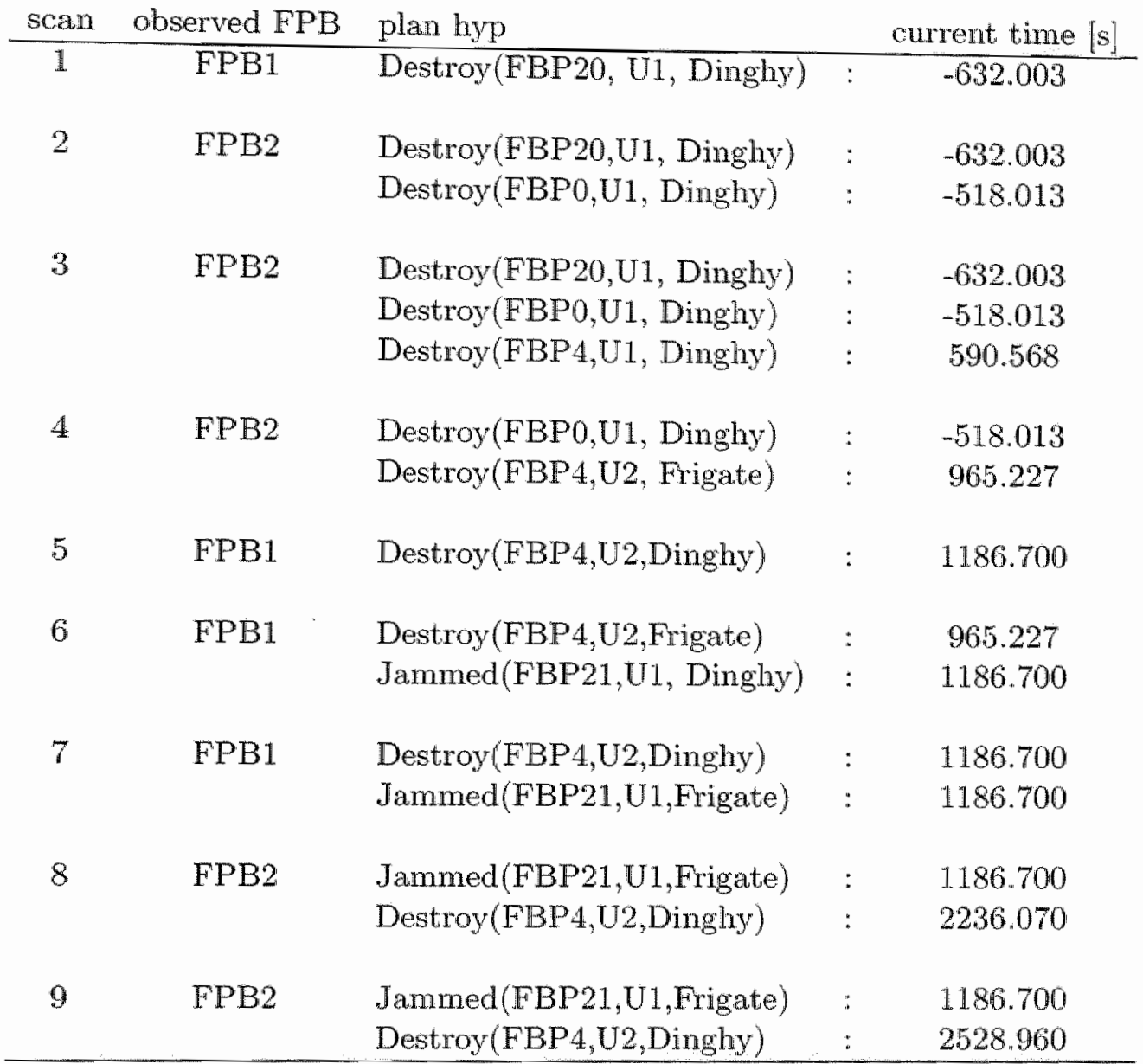

Table 11.12: The best assignment hypotheses of the littoral example after tuning.

\begin{tabular}{l|llll} 
helicopter & $\begin{array}{l}\text { separate } \\
\text { target }\end{array}$ & $\begin{array}{l}\text { first correct } \\
\text { plan }\end{array}$ & $\begin{array}{l}\text { number correct } \\
\text { plan }\end{array}$ & $\begin{array}{l}\text { track } \\
\text { continuity }\end{array}$ \\
\hline $\mathrm{H} 1$ & $0 / 5=0 \%$ & $0 / 5=0 \%$ & $5 / 5=100 \%$ & $3 / 5=60 \%$ \\
$\mathrm{H} 2$ & $0 / 4=0 \%$ & $2 / 4=50 \%$ & $2 / 4=100 \%$ & $4 / 4=100 \%$ \\
\hline
\end{tabular}

Table 11.13: Performance of the littoral scenario after tuning. 


\section{Chapter 12}

\section{Evaluation}

Now we have introduced the theories, design and implementation of a Tactical Plan Recognizer. In the previous chapter some experiments have been described. In this chapter we will further interpret the data of the experiments, evaluate the approach of this thesis and re-examine the software tools that have been applied here. The conclusions of the evaluation will be summarized in the final chapter on conclusions.

\subsection{Interpretation of the experiments}

In this section, the performance of the Tactical Plan Recognizer will be discussed. The following limitations of the Tactical Plan Recognizer 'surfaced' during the interpretation of experimental data.

- It is not really possible to recognize a complete plan ${ }^{1}$ in an early stage, but only partial plans. Only the parts that are being executed or that have been executed can be elaborated to some degree. Future plan parts can be modelled, either by a partial description of future parts or a number of plans that have equal earlier parts, but differ in parts that are executed in the final stages of the plan.

- In the Multiple Hypothesis Tracking (MHT) approach combined Deterministic Finite Automatas (DFAs) we have a good model for the events in the tactical world. However, not all parameters of the model will be

\footnotetext{
The terms complete and partial plans were introduced in section 4.1 .1
} 


\begin{tabular}{|l|c||c|}
\hline \multicolumn{2}{|c|}{ parameter } & value \\
\hline \hline old plan hypothesis time-out & $T_{\text {old }}$ & average plan duration \\
\hline number hypothesis retained & $\mathrm{k}$ & $k \geq N_{P L}$ Narget $^{\text {nom }}$ \\
\hline detection probability & $P_{D}$ & sensor model \\
\hline number of new plans/scan & $N_{N P}$ & performance tuning \\
\hline number of false plans/scan & $N_{F P}$ & sensor model \\
\hline process noise of the distance & $\sigma_{d}$ & performance tuning \\
\hline process noise of the speed & $\sigma_{v}$ & performance tuning \\
\hline
\end{tabular}

Table 12.1: Tuning parameters and the origin of their values.

known on forehand and, initially, only best guessed values must be used. For a set of 'learning' scenarios they can be tumed, until the recognizer has a good performance.

- Of course, the best assignment hypothesis has the highest probability, but the deviation (spread) of the assignment probabilities indicates the certainty of the the best assignment hypotheses. If it is difficult to decide between some high probability assignment hypotheses, they will have similar values of their probability.

\subsection{Tuning process}

As mentioned before, the tuning process consists of finding values for some tuning variables, such that the performance of the Tactical Plan Recognition system is sufficient. The most relevant parameters are given in table 12.1. The first column gives the turing parameter, in the next column the tuning criterium is given.

The minimal value of the k-best parameter is determined by the amount of targets, the amount of new plan hypotheses that are generated for each observation and the amount of observations that are processed during association. Worst-case, all targets are found in different assignment hypotheses. Now assume that it takes two scans for the targets to be clustered in fewer assignment hypotheses, than the $k$-best parameter is determined by the number of assignment hypotheses required for the amount of targets.

$$
k_{\min } \geq N_{P L}{ }^{N_{\text {target }}}
$$


Where: $k_{\text {min }}$ is the minimal $k$-best value, $N_{\text {target }}$ is the number of targets, $N_{P L}$ is the number of new plans created for each new target.

It is worthwhile to expound a little bit more the necessity and place of the tuning process in the whole set-up. It should be clear that we are not just traversing the the parameter space, but instead perform tuning only to restrict unwanted behavior of the basic Tactical Plan Recognition system. As such we fully comply with the established practice for improving the performance of complex systems.

\subsection{Software engineering approaches}

One of the pitfalls of research on software applications (such as the Tactical Plan Recognizer) is that the research focusses on, at the time popular, software engineering methods and tooling rather than on the application itself, in this case: 'agent-based' and 'model-based'. Of course, I was urged strongly by the department management of my firm to model each observed target, anthropomorphically (or at least naively) by one agent running as a separate computer process. My inclination is that it is more interesting to develop a suitable algorithm for Tactical Plan Recognition than just to make a agent-based system that 'does something".

Though there now is some body of research on multi-agent systems, see the work of Wooldrige [102], the 'model-based' approach has no body of research to back it (the only field I know of is model based diagnosis from Artificial Intelligence) and it may just turn out to be an empty phrase.

Another popular requirement, is the development of a real time Demonstrator, that is integrated with other Command and Control software. This forced me to develop Inter Process Communication interfaces between Haskell and other programs. It proved to be very difficult and time-consuming and was abandoned.

I was determined not to let the focus of this thesis be on the software engineering, since it is not really relevant for the research as such. Nevertheless, it is shortly discussed in this section. The software engineering tools used in this project were chosen for easy prototyping and suitability for the problem at hand. The programming languages, used here, were: 1) Haskell for design and implementing the Tactical Plan Recognizer and 2) Java/Swing for implementing the Simulation and Display environment. The two separate applications communicate through XML and ASCII data files. 


\subsubsection{Haskell and the Tactical Plan Recognizer}

The usage of Haskell purely functional programming language offers some huge advantages, but there are also some large disadvantages. We start with its advantages.

The advantage of the language is that algorithms, that are inherently recursive in nature and which data structures can be modelled with sets or lists and tuples, can be expressed very succinctly in Haskell. Thus it seems that the development and maintenance effort of a Haskell program is greatly reduced.

However, this may not be true for very complex systems. Debugging a complex Haskell program is very difficult. The sequence of program steps is often not directly clear, due to its lazy evaluation algorithm and it is very difficult to supply debugging information to the programmer. In fact, all debugging information should be part of the output of the functions debugged. As a consequence, providing debugging information of intermediate functions, requires all debugging information to be in the function's return data and this has to be propagated through all higher level functions until the main, level 0 , function. Finally, the modelling of large records, as found in most complicated applications, with tuples is not really legible and the data records offered by Haskell are rather cumbersome.

There are many other functional languages around. A functional language that has some debugging functions is Erlang, but this language does allow side-effects in functions and therefore is not a purely-functional language. Languages such as LISP and SCHEME certainly are not purely-functional languages, and do not ever offer lazy evaluation. Therefore they are far more primitive in their expression evaluation.

To develop a larger scale Haskell program, requires very good design, based on formal methods and extensive unit testing of lower and intermediate level functions. Thus the need for debugging the entire system at once is reduced.

Interfaces in functional languages require the programmer to circumvent the lazy evaluation. Though some methods are available now (Monads in Haskell) it remains to be seen whether complex input/output is implemented easily in a functional language. For example, some graphical interfacing systems are availlable, but these are complex. Though some research is done in this area, its results are not very popular are not industry standard.

So the conclusion is that Haskell is a very nice and clean programming language, that is suitable for inherently recursive functions with sets or lists. It certainly useful for the Tactical Plan Recognizer, but for the Simulator and 
display software Java has been applied.

\subsubsection{Simulator, Human Computer Interface and Java/Swing}

Java is very nice language, and is under constant development and improvement by the Java Community Process. An advantage of the java language consists of the easy availability of a large number of libraries, either part of the Java Standard Edition or from public domain projects. In this project, the Swing GUI library and the JDOM XML reader software have been applied.

A downside of Swing, is its complexity. The classes of Swing are quite low level. Thus it necessitates the development of an, easier to use, 'layer' of high level classes that are compositions of the original Swing classes. Another disadvantage is that, at the time of the Simulator's development, there was not an easy-to-use GUI builder program available. The available GUI builders (in 2002) tried to exploit all possibilities of the Swing classes, thus resulting in rather complicated tooling.

However, after having grasped some insight in Swing, it does indeed offer a large scale of facilities. By example: Swing's 2D image display classes are easily used to display JPEG pictures of the simulated geographical situation of the Army and Littoral scenarios.

JDOM $[51]$ is a popular XML reader for Java ("Java DOM"). It is easy to use and offers full DOM functionality. The reader generates a dynamical data structure, that can be accessed by the Java Program. In our case, the XML files contains the contents of the 'Track Database', that is read by JDOM and stored in the actual Simulator's Track Database by application software that accesses JDOM's dynamical data structure.

The next chapter confronts our work with the problem statement and draws some conclusions. 


\section{Chapter 13}

\section{Conclusions}

This last chapter about conclusions first recalls the problem statement. The problem statement consists of four items. The conclusion about the results are discussed separately for each item. Finally the remaining issues and future work are given and discussed.

\subsection{The problem statement revisited}

Now recall the problem statement:

Can a sound, complete, optimal and feasible software system be devised for the Tactical Plan Recognition problem, that can handle unidentified observations directly?

The main question is: whether there is an optimal method that can handle unidentified observations directly, rather than designing a Tactical plan recognizer that applies a correlator followed by an identified observation plan recognizer. Once such an method is available, the next question concerns feasibility. Can de method be translated to an algorithm? Once the algorithm is available, we want it to have some useful requirements: it is sound and it is complete. Two contributions of the research were: 1) application of the MHT approach to Tactical Plan Recognition and 2) the adaptation of formal properties, that are based on classical abduction, to our Tactical Plan Recognition case. From this a problem statement was defined, that consisted of the items that are discussed in the following subsections. 


\subsubsection{A direct and optimal method}

The main question of the problem statement is addressed by applying Reid's formula (formula 5.5) for our Tactical Plan Recognition problem. The terms of the formula are simply redefined to our Tactical Plan Recognition case. Refer to section 5.3.2. The suitability of the algorithm is argued by three paradigmatic examples: $2.1,2.2$ and 2.3 .

Reid's formula gives the probability of each output assignment hypotheses and an optimal algorithm will give the $k$-best, see equation 5.7. Our Haskell algorithms apply a brute force search strategy, but the number of internal assignment hypotheses that are retained is pruned to a reasonable value, e.g. 10 , but this parameter has to be tuned for a particular tactical situation.

\subsubsection{A feasible method: the algorithm}

The second question of the problem statement is addressed by the algorithm development of section 9.2. An algorithm has been defined by Reid that works for limited sized problems. A problem is its computational complexity. For systems with many observers and multiple observations contributing to the data of a scan, it is not possible to apply Reid's data association algorithm directly. An improvement of Reid's association algorithm is found in the application of Murty's k-best algorithm. In Mulder et.al., [62], Murty's algorithm is adapted for sensor data fusion. Still the algorithm only can handle scenarios with about two observers and about 10 targets. To improve on this, a first attempt is made by Krüger, [45] and Sibma [79], who applied branch and bound techniques.

\subsubsection{A sound and complete algorithm}

We also want to check the 'correct' functioning of the algorithm; whether the algorithm is sound and complete. We did not address the termination property in this document.

For this one has to precisely define what is meant by the terms sound and complete. For this it is necessary to formally define the input and output of the Tactical Plan Recognizer. This is done in definition 5.3. Here a formal definitions of Tactical Plan Recognition in sections 5.3 for independent plans is given. In Mulder and Voorbraak [66] the formal definition for dependent plans can be found. For this introductory text, we ignore the dependent case and apply the formal definition to various examples. 


\subsubsection{Conclusion on the algorithm development}

At this stage of the research, the algorithms have been developed and it has been argued that they function correctly, see sections 8.2.3 and 8.2.4 for the identified observation recognizer and section 9.2.2. However, all the work on formal definitions and correctness of the algorithms, is rather academic. These features are 'nice to have', however, they do not guarantee useful results in an implemented system. The MHT model still is a rather coarse model of problem of Tactical Plan Recognition. Some parameters of Reid's formula are not well known and are open for tuning. Therefore we need to evaluate the performance of the implemented algorithm. If the initial model and initial set of parameters does not yield satisfactory results, tuning has to be done.

Remaining issues are:

- Recognition of goals is quite well possible, however, recognition of future plan parts is not possible. During ingress of a target, the egress cannot, be recognized, but only hypothesized.

- Switching between assignment hypotheses, leads sometimes to frequent changes of the best plan hypotheses.

- Only the best plan hypothesis does not suffice, but e.g. the best plan hypotheses of a number of likely assignment hypotheses have to be outputted.

- Some initial proposals for Human Computer Interfaces have been given. But for an actual product 'Tactical Plan Recognizer', further study and refinement for these interfaces will be required.

\subsection{Future research}

The current implementation enumerates all possible assignment hypotheses at a point of time. The size of the tree is exponential with the amount of plots and the amount of tracks in the system. Future work should, surely, include application of Mulder's, [62] and Krüger's [45] work on optimal k-best assignment algorithms in the current plan recognizer. This in order to be able to cope with larger problems.

Recall table 4.2 that shows the characteristics of plans that have been used in this work. The types of plan hypotheses is rather limited: 1) non- 
hierarchical and 2) complete plans. Future work might: 1) consider hierarchical plans. Here the techniques from inductive learning, such as the Version Space Learning systems might be applicable. 2) The complete plan hypotheses might not be suitable for all Tactical Plan Recognition problems and one might consider posing partial plan hypotheses, that are elaborated as new observations are entered into the plan recognizer.

The Tactical Plan Recognizer must still be extended to handle uncertain observations. An approach is to define different false alarm rates for different observation confidences.

The Tactical Plan Recognizer has been designed for unidentified observations, however sometimes identification information of targets is available. The recognizer does not integrate identified and unidentified observation plan recognition yet. In similar cases in tracking, where a sensor gives some identification information, the correlation function has been adapted.

Only a limited set of scenarios, to test the Tactical Plan Recognizer, has been available yet. The number of test scenarios certainly should be increased if one wants to make the algorithms 'product ready'. But experience shows that it is very difficult to acquire detailed military knowledge. Some initial requirements for such a product are listed in appendix $B$.

To describe identification uncertainty logically, the 'there is a' quantifier ( $\exists$ ) was used. But this does allow multiple objects to be substituted to the quantified variable, where we might really want only one object to be instantiated to the quantified variable. The 'there is only one' quantifier ( $\exists !$ !) does make the latter statement about variable substitution, but it has not been used here. The reason for this was lack of an adequate deduction system. Future research might encompass and utilize this quantifier.

Some initial proposals for Human Computer Interfaces have been given in this thesis. But it is also clear that the assignment hypotheses and their constituent plan hypotheses can be fairly complex. This complex information has to be communicated to an operator, who may be under stress. To attain a product-ready Human Computer Interface for this will require some study.

The method for generating plan hypotheses, that is proposed and implemented in this research, applies a traditional planning algorithm and therefore a sequence of goal recognition and planning is applied. From assumption 5.1 105 we know that the plan will contain at least an action that will unify with the first observation. This may give a handle to develop a planning algorithm that inputs the first observation and directly generates plan hypotheses. 
With this work a novel way of automated Tactical Plan Recognition has been introduced. Whereas previous authors and systems did pay little attention to the fact that in a military situation most observations of enemy targets are unidentified, it is the focus of this research. Because of these unidentified observations, the task is similar to that of Multi Target Multi Sensor tracking, where the Multiple Hypothesis Tracking algorithm of Reid has been applied very successfully. Therefore, the algorithm of Reid has been modified in this research to perform Tactical Plan Recognition. The other focus of this research concerns the application of abduction in formal definitions of Tactical Plan Recognition. Previous authors on Tactical Plan Recognition did not give a formal definition and hence could argue that their algorithms were sound and complete. By means of formal definitions of this research, it is possible to argue that the algorithm has these properties. 


\section{Appendix A}

\section{Basic terminology}

Basic terms of the military world and Tactical Plan Recognition are (adapted from Bernstein, [13]):

- Plot, Measurement: a position and speed measurement, by a sensor, of a target in the outside world.

- Observation, Human Intelligence: a position and heading measurement of a target in the outside world, by a human observer, in the outside world.

- Correlation: computing the similarity of a measurement, or information.

- Association, Assignment: deciding on which data could have been caused by the same target.

- Tracking: creating and updating target state estimates by associating sensor measurements over time using a predictive model of target dynamics.

- Classification: determining that a perceived target is of general or specific type.

- Identification ${ }^{1}$ : recognizing a perceived target as a particular individual.

\footnotetext{
${ }^{1}$ whether the identification of a target of an observed object is known or unknown turns out to be crucial for the Tactical Plan Recognition algorithm
} 
- Situation assessment: inferring relations among entities, to include (own) force structure and cross force relations, communications, physical context, etc.

- Threat assessment: significance assessment regarding detected or predicted hostile situations, to include force susceptibilities and vulnerabilities to enemy postures, intentions and courses of action.

Formal properties of algorithms, that are discussed in this report, are:

- Soundness: an algorithm is sound, if every solution it produces is indeed a correct solution of the problem it is designed to solve.

- Completeness : an algorithm is complete when it generates a solution if the solution exists.

- Termination : an algorithm terminates, given a finite input.

- Computational complexity : this is the order of number of processing steps.

- Memory complexity : this is the order of the size of the required memory. 


\section{Appendix B}

\section{Requirements on a Tactical Plan Recognition product}

In this appendix some initial requirements on a Tactical Plan Recognition product are given.

1. Requirement on an observed object's hypothetical plans. It has been shown that the Tactical Plan Recognizer, presented here, will generate all hypothetical plans, given Azarewicz's first closed world assumption.

2. Requirement on an observed object's intentions. We have seen that the problem definition function of the Tactical Plan Recognizer generates possible goals. Given the background knowledge, a rule-based system will generate all possible goals that apply for the object.

3. Requirement on identified observations. In this research, a separate plan recognizer for identified observations has been defined. So recognition of identified observations has not been integrated yet in the Tactical Plan Recognizer.

4. Requirement on uncertain parameter values of observations. Our Tactical Plan Recognizer uses statistical measures in the calculation of an assignment hypothesis its probability.

5. Requirement on multiple plans. The plan recognizer can certainly handle multiple plans, each executed by a different object. The information about different objects are organized in 'tracks'. 
6. Requirement on multi sensor/observer. Indeed multiple observers have been simulated. In figure 10.13 and example situation is displayed.

7. Requirement on ranked plan hypotheses.Our Tactical Plan Recognizer ranks the assignment hypotheses by Reid's formula and outputs the kbest assignment hypotheses, with their probability. 


\section{Appendix $\mathrm{C}$}

\section{XML schema for assignment hypotheses}

The communication of assignment hypotheses between the Tactical Plan Recognizer and the Simulator program is done through "*tpr.xml" XML files. The syntax of such a file is defined by the following XML schema.

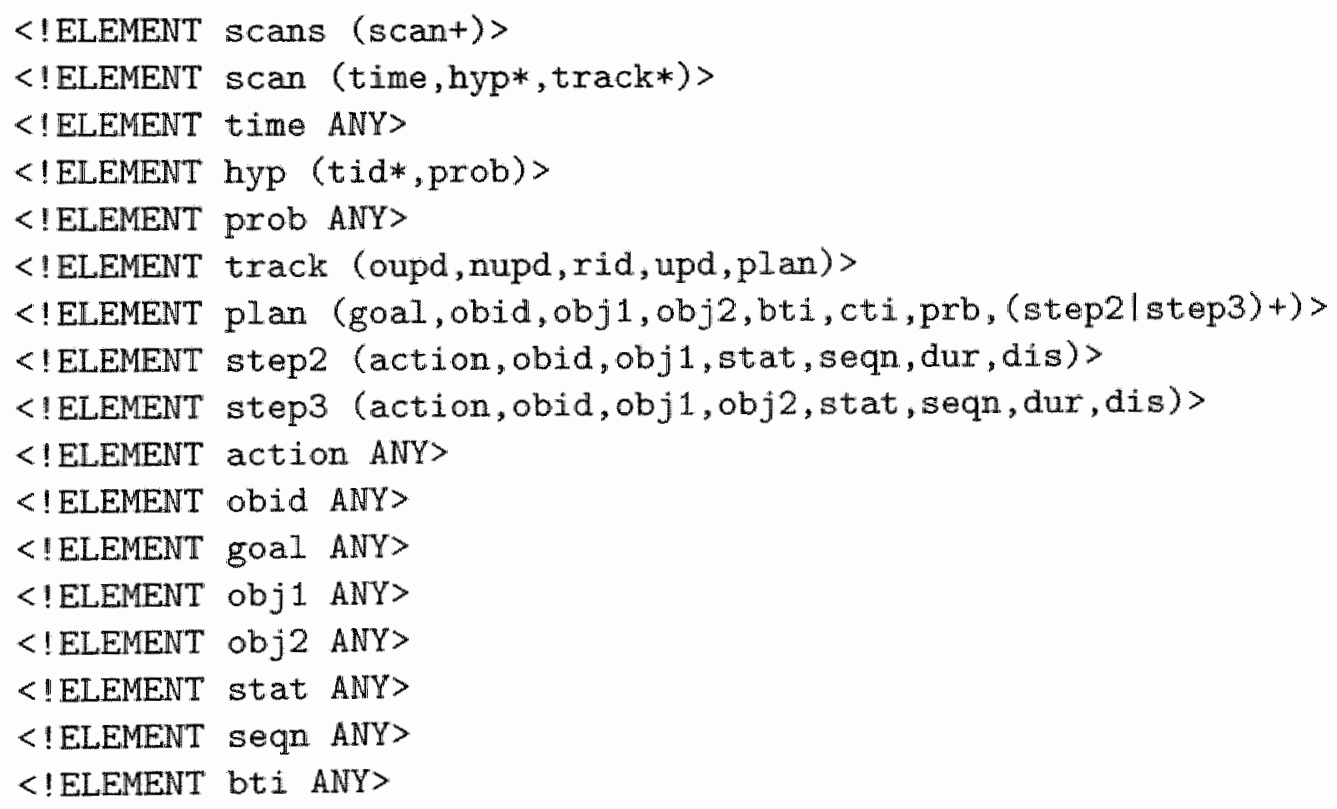


242 APPENDIX C. XML SCHEMA FOR ASSIGNMENT HYPOTHESES

<IELEMENT cti ANY> $<$ ELEMENT dur ANY> <IELEMENT dis ANY> $<$ IELEMENT prb ANY <!ELEMENT tid ANY> $<$ ELEMENT upd ANY> $\langle$ !ELEMENT rid ANY> $<$ IELEMENT oupd ANY> $<$ IELEMENT nupd ANY > 


\section{Bibliography}

[1] L. Albert. Average case analysis of the rete pattern matching algorithm and average size of join in databases. In Foundations of Software Technology and Theoretical Computer Science, Bangalore, India. Springer Verlag, 1989.

[2] L. Albert and F. Fages. Average case analysis of the rete pattern matching algorithm. In $15^{\text {th }}$ International Colloquium on Automata, Languages and Programming, Lecture Notes in Computer Science, $31 \%$. Springer Verlag, 1988.

[3] A. Aliseda-LLera. Seeking Explanations: Abduction in Logic, Philosophy of Science and Artificial Intelligence, PhD thesis Stanford University. ILLC Dissertation Series 1997-4. Institute for Logic, Language and Computation, Amsterdam, 1997.

[4] J.F Allan, H.A. Pelavin, and R.N. Tenenberg. Reasoning About Plans. Morgan-Kaufmann, San Mateo, Cal., 1991.

[5] L. Ardissono. Dynamic User Modeling and Plan Recognition in Dialogue. PhD thesis, Universita" di Torino, Italy, 1996.

[6] L. Ardissono and S. Sestero. Using dynamic user models in the recognition of the plans of the user. In User Modelling and User-Adapted Interaction, pages 157-190. Kluwer Academic Publishers, 1996.

[7] J. Azarewicz. Plan recognition for airborne tactical decision making. In Proceedings of the fifth National Conference On Artificial Intelligence (AAAI-86), pages 805-811, 1986. 
[8] J. Azarewicz and et.al. Template-based multi-agent plan recognition for tactical situation assessment. In Proceedings on the fifth Conference on Artificial Intelligence Applications, 1989.

[9] M. Bares. Pour un Perspective des Systemes de Commandement. Polytechnica, Paris, 1996.

[10] M. Bauer. Integrating probabilistic reasoning into plan recognition. In Proceedings of the tenth European Conference on Artificial Intelligence (ECAI-94), pages 620-624, 1994.

[11] M. Bawer. Machine learning for user modelling and plan recognition. In Working Notes on the ICML-96 Workshop: ML meets HCI, 1996.

[12] M. Bauer and G. Patu. Logic-based plan recognition for intelligent help systems. In E. Sandewal Backstrom, C., editor, Current Trends in AI Planning, pages 60-73. IOS Press, 1994.

[13] A.N. Bernstein. Multi Sensor Data Fusion and Multi Target Tracking. H. Silver and Associates, 1998.

[14] R. Bird. Introduction to Functional Programming using Haskell. Prentice Hall Europe, 1998.

[15] M. Boasson. Trends in naval command and control systems. In Revue Technique. Thomson-CSF, 1994.

[16] Y. Boers, J.N Driessen, and N. Laclé. Automatic track filter tuning by randomized algorithms. IEEE Transactions on Aerospace and Electronic Systems, vol. 38, no. 4, 2002.

[17] J. R. Boyd. A Discourse on Winning and Losing. Air University, Maxwell Air Force Base, Alabama, USA, 1987.

[18] T. Bylander. The computational complexity of propositional STRIPS planning. Artificial Intelligence, 69, 1994.

[19] R.J Calistri-Yeh. Classifying and detection of plan-based misconceptions for robust plan recognition. AI magazine, 12(3), 1991.

[20] D. Canamero. A knowledge-level approach to plan recognition. In Proceedings of the IJCAI-95 Workshop on Plan-Recognition, Montreal, 1995. 
[21] D. Canamero. Plan recognition for decision support. In IEEE Expert, June 1996, 1996.

[22] S. Carberry. Plan Recognition in Natural Language Dialogue. MIT Press, Cambridge MA, 1990.

[23] B. Chandrasekaran. Generic tasks in knowledge-based reasoning: high level building blocks for expert system design. IEEE Expert, 1984.

[24] E. Charniak. Towand a Model of Children's Story Comprenension. Technical Report, AI-TR-266. MIT press, Cambridge, Mass., 1972.

[25] E. Charniak and R.P. Goldman. A probabilistic model of plan recognition. In Proceedings Ninth National Conference on Artificial Intelligence (AAAI-91), volume Vol. 1, pages 160-165, 1991.

[26] Naval Reserve Officers Training Corps. Process of naval command and control. 2003.

[27] R.E. Cullingford. Script application: computer understanding of newspaper stories. Technical report, Yale University, New Haven, Conn., 1978.

[28] R.E. Cullingford. SAM, pages 627-649. Morgan-Kaufmann, Los Altos, Cal., 1986.

[29] J.N. Driessen, F.W. Mulder, and F.C.C.B. Rulof. Msdf-lib: A generic softwrare library for developing operational target tracking applications. In Proceedings of the Symposium on "Target Tracking and Sensor Data Fusion for Military Observation Systems" (SET-59). RTO Symposium, Budapest, HUNGARY, 2003.

[30] R. E. Fikes and N.J. Nilsson. Strips: A new approach to the application of theorem proving to problem solving. Artificial Intelligence, 2:189-208, 1971.

[31] J. Genesereth and N.J. Nillson. Logical Foundations of Artificial Intelligence. Morgan Kaufmann, San Mateo, Cal., 1987.

[32] M.P. Georgeff and A. Lansky. Reactive reasoning and planning. In Proceedings of the Sixth International Conference on Artificial Intelligence, IJCAI87, San Mateo, Cal., 1987. Morgan Kaufmann. 
[33] M.P. Georgeff and A. Lansky. Reactive reasoning and planning: an experiment with a mobile robot. In Proceedings of the Sixth National Conference on Artificial Intelligence (AAAI-87), Seattle, WA, pages 677$682,1987$.

[34] C.J. Harris. Application of Artificial Intelligence to Command \& Control Systems. Peter Peregrinus Ltd, London, on behalf of the Institution of Electrical Engineers, 1988.

[35] C.J. Harris and I. White. Advances in Command, Control and Communication Systems. Peter Peregrinus Ltd, London, on behalf of the Institution of Electrical Engineers, 1987.

[36] B. Hayes-Roth. A blackboard architecture for control. Artificial Intelligence, 26, 1985 .

[37] B. Hayes-Roth and et. al. A layered environment for reasoning about action. Technical report, Stanford University, 1986.

[38] C. Heinze, S. Goss, and A. Pearce. Plan recognition in military simulation: Incorporating machine learning with intelligent agents. In Proc. of IJCAI-99 Workshop on Team Behaviour and Plan Recognition, pages $53-64,1999$.

[39] M.A.W Houtsma. Advanced situation assessment support in maritime $c^{4} i$ systems. In Proc. of the NATO symposium Norfolk 2002, 2002.

[40] M. J. Huber and E. H. Durfee. Observational uncertainty in plan recognition among interacting robots. In Working Notes of the IJCAI-93 Workshop on Dynamically Interacting Robots. Elsevier Science Publishers, 1993.

[41] A.H. Jazwinski. Stochastic Processes and Fittering Theory. Academic Press, New York and London, 1970.

[42] M.P. Jones and J. Peterson. The Hugs 98 User Manual, 1999.

[43] E. Jong, de. Mht implementation in haskell. personal communication, 1994. 
[44] H.A. Kautz and J. Allan. Generalized plan recognition. In Proceedings of the Fifth National Conference on Artificial Intelligence, AAAI-86, pages $32-37,1986$.

[45] T. Krüger. The Multiple Sensor Multiple Target Data Assaciation Problem with Additional Constraints. Thales Nederland B.V. and University of Twente, 2003.

[46] F.W. Lanchester. Aircraft in Warfare - The Dawn of the Fourth Arm. Constable and Co., London, United Kingdom, 1916.

[47] J.H.J. Lenting and P.J. Braspenning. The frame problem from an engineering perspective. International Journal for Software Engineering and Knowledge Engineering, 3:257-265, 1993.

[48] N. Lesh and O. Edzioni. Insights from machine learning for plan recognition. In IJCAI-95 Workshop on the Next Generation of Plan Recognition. Systems., 1995.

[49] V.R. Lesser. The distributed vehicle monitoring testbed: a tool for investigating distributed problem solving networks. AI-magazine, $4(3): 15-33$, 1983.

[50] D. Lin and R. Goebel. A message passing algorithm for plan recognition. In Pracedings of the Twelfth Intemational Conference on Artificial Intelligence (IJCAI-91), volume Vol. 1, pages 280-285, San Mateo, Cal., 1991. Morgan Kaufmann.

[51] B. McLaughlin. Java and XML. O' Reilly and associates, 2000.

[52] O.M. Mevassvik and A. Lokka. Fusion of radar tracks, reports and plans. In Proceedings of 3 rd International Conference on Information Fusion. International Society of Information Fusion, 2000.

[53] F.W. Mulder. Generalized multiple hypothesis tracking, ass-memo w08.1994. Technical report, Hollandse Signaalapparaten B.V., 1994.

[54] F.W. Mulder. Theory of and algorithms for deleted track-initiated track correlation in musician, asr-memo w10.1994. Technical report, Hollandse Signaalapparaten B.V., 1994. 
[55] F.W. Mulder. Tuning van de track-evaluatie functie van musician (tuning of the track-evaluation function of musician), asr-memo w17.1994. Technical report, Hollandse Signaalapparaten B.V., 1994.

[56] F.W. Mulder. A generic task model for tactical plan recognition in a naval c\&c system. In Proceedings of the 15 th World Computer Congress, Vienna/Budapest. International Federation of Information Processing (IFIPS), 1998.

[57] F.W. Mulder and M. Boasson. Een gedistribueerd blackboard systeem gebaseerd op splice/xia-c (a distributed blackboard system based on splice/xia-c). In Proc. of NAIC/AIT'91, Best Paper Award AIT"91, 1991.

[58] F.W. Mulder and M. Boasson. A loosely coupled distributed blackboard system for approach control. In Proc. International Conference on Artificial Intelligence, Avignon, pages 459-471, 1993.

[59] F.W. Mulder and P.J. Braspenning. A complex blackboard system for image-sequence interpretation. In Proceedings AI Toepassingen '88 (AIT'88), Best Paper Award AIT'88 (In Dutch). Academic Service, 1988.

[60] F.W. Mulder and P.J. Braspenning. Anallysis of multi-granular planning, in dutch. In Proceedings NAIC-89, (eds. L.Siklossy and R.R. Bakker), pages 3-14. Academic Service, 1989.

[61] F.W. Mulder and P.J. Braspenning. A generic task model for tactical plan recognition. In Proc of the Sixteenth International Conference Software 8f Systems Engineering B their Applications (ICSSEA-2003), 2003.

[62] F.W. Mulder, J.H. Driessen, F. Rulof, and P. Verveld. Advanced sensor data fusion in msdflib. In International Conference on Information Fusion, Cairns, Australia in 8-11 July 2003, 2003.

[63] F.W. Mulder and J.G. Pointeau. Kinetic estimation in plan recognition. In Innovation Report Series. Thales Nederland B.V., 2003.

[64] F.W. Mulder, M.J. Steenkamer, P.J. Braspenning, and P.E. van der Vet. A generic task model of distributed reactive plaming and its application 
to approach control. In Working Papers NAIC'93, eds. J.M. Akkermans and J.A. Breuker, pages 79-90, 1993.

[65] F.W. Mulder and F. Voorbraak. A formal description of tactical plan recognition, cr-s-01-01. In Interne rapportenreeks Communications Research and Semiotics, ISSN 1567-8989. Section Communications Research and Semiotics, Faculty of General Sciences, University of Maastricht, 2001.

[66] F.W. Mulder and F. Voorbraak. A formal description of tactical plan recognition. In Information Fusion Journal, volume 4, pages 47-61. Elsevier Science Publishers, 2003.

[67] K. G. Murty. An algorithm for ranking all the assignments in order of increasing cost. Operations Research, vol 16, 1967.

[68] R.E. Neapolitan. Probabilistic Reasoning in Expert Systems: Theory and Algorithms. Wiley, 1990.

[69] D.F. Noble. Template-based data fusion for situation assessment. In Proc. Tri-Service Data Fusion Symposium, pages 152-162, 1987.

[70] P.S. Ow and et. al. Reactive plan revision. In Proceedings of the Seventh National Conference on Artificial Intelligence (AAAI-88), San Mateo, Cal., 1988. Morgan Kaufmann.

[71] D.V. Pynadath and M.P. Wellman. Accounting for context in plan recognition, with application to traffic monitoring. In Proceedings of the Fourteenth National Conference on Artificial Intelligence (AAAI95). Morgan Kaufman, 1995.

[72] A. Quilici. A hybrid approach to recognizing programming plans. In Proceedings of the First Working Conference on Reverse Engineering (WCRE-93), pages 126-133, Baltimore MD, 1993. IEEE press.

[73] A. Quilici, Q. Yang, and S. Woods. Applying plan recognition algorithms to program understanding. In Proceedings of the Eleventh Annual Knowledge-Based Software Engineering Conference, Sept 1996, pages 99-103, Syracuse NY, 1996. IEEE press.

[74] D.B. Reid. An algorithm for tracking multiple targets. IEEE Transactions on Automatic Control, Vol. AC-24, No. 6, December 1979, 1979. 
[75] K.N. Ross and R.D. Chaney. Hidden markov models for threat prediction fusion. In Belur V. Dasarathy, editor, Proceedings of SPIE, Sensor Fusion: Architectures, Algorithms, and Applications IV, volume Vol. 4051. SPIE, 2000.

[76] S.J. Russel and P. Norvig. Artificial Intelligence: A Modem Approach. Prentice Hall, Englewood Cliffs NY, 1995.

[77] R.C. Schank and R.P. Abelson. Scripts, Plans, Goals and Understanding. Erlbaum, Hillsdale NJ, 1977.

[78] C.F. Schmidt, N.S. Sridharan, and J.L. Goodson. The plan recognition problem: an intersection between psychology and artificial intelligence. Artificial Intelligence, 11:45-83, 1978.

[79] B. Sibma. Hypotheses for the multiple sensor multiple target data association problem: $k$-best assignment algorithm with additional constraints. Master's thesis, University of Groningen, 2004.

[80] C. Sidner. Plan parsing for intended response recognition in discourse. Computational Intelligence, Vol. 1:1-10, 1985.

[81] R.W. Sittler. An optimal data association problem in surveillance theory. IEEE Transactions on Military Electronics, Vol. AES-11, April 1964:125-139, 1964.

[82] D.F. Stanat and D.F McAllister. Discrete Mathematics in Computer Science. Prentice-Hall International Editions, 1977.

[83] R. Suzic. Generic representation of military organisation and military behaviour: Uml and bayesian networks. In RTO Symposium on C3I and MBS Interoperability, Oct 2003. RTO, 2003.

[84] R. Suzic. Representation and recognition of uncertain enemy policies using statistical models. In RTO Symposium on Military Data and Information Fusion, Oct 2003. RTO, 2003.

[85] M. Tambe. Resc: an approach to agent tracking in a real-time, dynamic environment. In Proceedings of the International Conference on Multi Agent Systems (ICMAS), 1995. 
[86] M. Tambe and P.S. Rosenbloom. Event tracking in a dynamic multiagent environment. Computational Intelligence, 1996.

[87] D.S.W. Tansley and C.C. Hayball. Knowledge-Based System Analysis and Design. Prentice Hall, 1993.

[88] S. Thompson. The Croft of Functional Programming. Addison Wesley, 1996.

[89] M. Van Wie. Observation and cooperation, thesis proposal for the degree of doctor of philosophy. Technical report, University of Rochester, Dept. of Computer Science, 1998.

[90] M. Van Wie. Role Selection in Teams of Non-Cooperating Agents. PhD thesis, University of Rochester, Dept. of Computer Science, 2001.

[91] P. Verveld. Solving multiple target tracking using a k-best assignment algorithm. Master's thesis, Hollandse Signaalapparaten B.V., University of Twente, 1997.

[92] M. Villain. Getting serious about parsing plans: a grammatical analysis of plan recognition. In Proceedings of the Eleventh National Conference on Artificial Intelligence (AAAI-90), pages 190-197, San Mateo, Cal., 1990. Morgan Kaufmann.

[93] H. de Waard. Complexity of constrained hypotheses in mht. personal communication, 1994.

[94] Wikipedia. Wikipedia, the free encyclopedia, 2003.

[95] D. Wilkins. Recovering from execution errors in sipe. Computational Intelligence, $1: 33-45,1985$.

[96] L.M. Wills. Automated program recognition: a feasibility demonstration. Artificial Intelligence, Vol 45(2) February 1990:113-172, 1990.

[97] W.L Winston. Operations Research, Applications and Algorithms. Duxbury Press, Belmont Cal., 1991.

[98] R. Wols. Wp300, ntp-datafusie: Military expert knowledge base. Technical report, FEL-TNO, The Hague, 1995. 
[99] R. Wols. Wp3002, ntp-datafusie: The aggregation process. Technical report, FEL-TNO, The Hague, 1995.

[100] S. Woods, A. Quilici, and A. Deursen. Program plan recognition for year 2000 tools. In Proceedings of the Fourth Working Conference on Reverse Engineering (WCRE-97), October 1997, Baltimore MD, 1997. IEEE press.

[101] S. Woods, Q. Yang, and A. Quilici. Program understanding and plan recognition: a comparative study. Technical report, University of Waterloo, 1995.

[102] M. Wooldrige. MultiAgent Systems. John Wiley and Sons, Ltd., 2002.

[103] I. Zuckerman. Generation and selection of likely interpretations during plan recognition in task-oriented consultation systems. User Modeling and User-Adapted Interaction, Vol. 1:323-353, 1991. 


\section{Index}

Abduction, 68

Action, 11

Analysis Aspect

Control Problem, 111

Coordination Problem, 111

Domain Problem, 111

Assignment, 80

Hypothesis, 81

Automated Driving, 19

Azarewicz, 11, 20

Closed World Assumptions, 70

Bauer, M., 18

Bernstein, 22

Binary Relation

Parenthood, 102

Partial Order, 64

Quasi Order, 102

Total order, 64

Canamero, 20

Carberry, 20

Charniak, 20

Command and Control System

Matis Model, 25

Mulder's Model, 26

Command and Control System, 5

Boasson's Model, 25

Joint Directors of Labs Model, 22
Completeness, 151, 160,238

Complexity

Computational, 151, 238

Exponential, 127

Memory, 151, 163, 238

PSPACE-complete, 132

Consistency, 147

Constraint, 12

Dantzig, G., 10

Data Fusion Demonstrator, 39

DFA, 131

Doctrine, 9

Equivalence Class, 80

Examples

Army Example, 196

Forward Chaining, 126

RETE, 127

Generic Task Model, 22

PR with Ident. Obs., 111

PR with Unid. Obs, 113

Geographic Information System, 126 Goal

Recognition,Problem Definition, 123

Goldman, 20

Human Computer Interface, 172 
Intelligent Computer User Interfaces, 19

Java, 172

Applet, 179

JDOM, 229

Standalone Program, 179

Swing, 179, 229

Kalman Filter, 140

Kautz, H., 18

Kinematic Estimation, 133

Constant Velocity, 139

Mean Reverting Process, 137

Lanchester, F.W, 9

Lesh, N., 18

Matis, 25

Mean Reverting Process, 137

Mevassvik, 42

Military World, 14

Modus Ponens

Generalized, 126

Multi-Agent Coordination, 20

Multiple Hypothesis Tracking, 54

Natural Language Understanding, 19

Observability, $73,138,139$

Observation

Action, 73

Identified, 74, 82

Probabilistic, 75

State, 73

Uncertain, 75

Unidentified, 74,88

Operations research, 10
Plan

Complete, 65

Hierarchical, 64

Linear, 64

Partial, 65

Partial Order, 64

Skeletal, 64

Total order, 64

Plan Hypothesis

root id, 196

Plan Recognition

Formal Definition, 68

Intended, 65

Keyhole, 65

Probabilistic, 94

Plan Recognizer, 172

Deterministic, 79

Identified Observation, 143

Probabilistic, 79

Unidentified Observation, 153

Planning

Conditional, 9

DFA, 131

Distributed, 110

Enemy Forces", 9

Granularity, 63

Instantiation, 64

Own Forces', 9

Partial Order, 64, 129

Reactive, 9, 109

Total Order, 64

World, 11

Program Understanding, 19

Psychological Modelling, 18

Quilici, 20

Reid, Donald, 54, 95 
Resolution, 127

RETE, 127

Scenarios

Army example, 196

Littoral example, 214

Schmidt, C.F., 18

Sidner, C., 18

Simulator, 172

Basic Database, 175

Track Database, 175

Sittler, 54

Soundness, 160, 238

Soundness,Completeness, 149

SPLICE, ii

Termination, 238

Toy World, 13

Track Database, 177

Hyp class, 178

Scan class, 178

Track class, 178

Track's root id, 178

Universal Instantiation, 132

Villain, M., 18

Wols, 20

World State, 11

XML, 174

XML schema, 174 


\section{Summary}

Plan recognition can roughly be described as the problem of finding the plan(s) underlying the observed behavior of agent(s). In the case of Tactical Plan Recognition, one wants to recognize the enemy's military plan. Information for this comes from human intelligence and sensors. These produce observations of enemy objects. An observation has information about the current position of an enemy object, its speed and direction and, possibly, its actions. With the observations, only an incomplete 'picture' of the military world can be created; one does only have limited knowledge! Because of incomplete knowledge of the military world, a plan recognizer can, at best, only generate some reasonable plan hypotheses about the situation.

Traditionally, plan recognition has been studied, formalized and implemented in areas like story understanding and user modelling. This dissertation focusses on military applications, where this task of tactical plan recognition is crucial. However, this task is relevant for every application where one has to deal with adversarial agents.

Tactical plan recognition differs from traditional plan recognition in a number of ways. An enemy will often try to avoid making his plans known. Only partial achievement of planning goals can occur here. There are multiple independent or related plans and one is faced with a mult:i target multi sensor data fusion problem. 'This work focusses on a particular consequence of the covertness of the enemy's military operation: namely that the identity of the observed enemy objects, for which plans are to be recognized, is unknown. A consequence of this is that it is typically not known which observations originate from the same objects. Moreover the kinematic state of the object can only be estimated because the position and speed measurements are uncertain and are generally modelled by stochastic variables.

Other systems for this problem consisted of a sequential correlator and plan recognition function. The correlator relates successive unidentified ob- 
servations that are thought to originate from the same agent. Thus identified observations are created, which then are input for a traditional identified observation plan recognizer. Disadvantages of this approach are: 1) that not all information that is available in a plan hypothesis is actually used for correlation and 2) not all possible combinations of observations are considered by the correlator. So actually a direct handling of unidentified observations in one algorithm is preferred.

In designing algorithms, it is beneficial to be able to make statements about their correctness. In other words: one wants to discuss the soundness and completeness of the algorithm. For this a formal description of Tactical Plan Recognition is required, but this has not been stated in other work.

This work then addresses the following research question:

Can a sound, complete, optimal and feasible software system be devised for the Tactical Plan Recognition problem, that can handle unidentified observations directly?

The formalization of Tactical Plan Recognition presented here is based on classical abduction. The concepts of classical abduction can readily be applied to plan recognizers for identified observations. However, for Tactical Plan Recognition some adaptations have to be made. Here the plan recognizer will not only have to generate plan hypotheses, but also assignment hypotheses. These assignment hypotheses relate observations with hypothetical plans. A choice for an assignment is essentially a decision concerning the question which observations originate from the same objects. Now the formal description is available, it is readily applied for definitions of the soundness and the completeness of the algorithms.

For observations with stochastic variables the probability of an assignment hypothesis is calculated, rather than the probability of the plan hypotheses. The latter method is found in traditional Tactical Plan Recognizers. For this, Reid's multiple hypothesis tracking formula can be adapted to calculate the assignment hypothesis probability.

The formall definitions and the application of Reid's formula are illustrated by a large number of examples of tactical situations. They concern military settings and one civil aviation setting. The examples start with simple identified observation cases, till the difficult unidentified observations case. In the final example, one finds unidentified observations with stochastic variables for kinematic measurements. 
The formal definitions give a very precise description of the input of the Tactical Plan Recognizer and relate the input to the output. The next phase in the development of an algorithm consists of the analysis of the Tactical Plan Recognition problem. This is done by proposing a Generic Task Model for the problem. A Generic Task Model describes a class of problems, rather than individual instantiations of the problem. Of course, in our case it concerns the class Tactical Plan Recognition. Instantiations of this class are specific systems developed, e.g. the Data Fusion Demonstrator that operated in the army domain. Two Generic Task Models are presented: one for the simpler problem that inputs identified observations, followed by one for the problem at hand, Tactical Plan Recognition by unidentified observations.

Once the Generic Task Model is available, algorithms can be developed. In this thesis, the algorithms are specified in the high level and purely functional Haskell language. First some suitable algorithms for the problem definition and planning functions are selected. Then a suitable model for the estimation of kinematic parameters is selected. It turns out that a straight uniform motion model does a much better speed estimation than the reverting model of Mevassvik. Then the complete plan recognition algorithms are given. As is done in the analysis phase, we start with simpler case of identified observations. For this case, a generate and test algorithm is presented. Then its soundness and completeness are discussed. Also its complexity is examined.

The identified observation algorithm was still fairly simple, but it is followed by the, rather complicated, unidentified observation Tactical Plan Recognition problem. It has some parts have already been presented in the identified observations algorithm. The discussion of soundness and completeness, is based on the conclusion of the first algorithm. With regards to the complexity of the unidentified observation algorithm, the focus is on the memory complexity. A description of the amount of assignment hypotheses that are handled is given as a function of the number of observations handled by the algorithm. The complexity is very large; in fact it is Non Polynomial. The result is that hypothesis management is required (pruning, data partitioning) and only small problem sizes can be handled.

The last part of the thesis concerns and evaluation of the algorithms. This is done by means of a Test and Evaluation software. It consists of a Java Simulator and Display program and the described Haskell implementation of a Tactical Plan Recognizer. They communicate through XML and ASCII data files. Two scenarios are used for evaluation: the first is the army example 
(presented earlier), the second is a littoral scenario.

The army example is studied in detail. This example concerns two army attack helicopters that attack different enemy units. The assignment hypotheses are examined after entering each observation. Their probabilities and the best assignment hypothesis are discussed. In this example one finds an assignment hypothesis with an initially low probability, but with the maximal probability some observations further. So it makes a point for retaining a significant number, e.g. 10, assignment hypotheses after each reduction (pruning of assignment hypotheses). The performance is not very good: the two targets are not always recognized as two separate targets and the track continuity is minimally $33 \%$.

To improve on the performance, some parameters are tuned for the scenario. The practice of tuning is always found in applications, where the models of the outside world are limited in detail. The quality of the set of correlations is improved by restricting the correlation windows. The new plan density parameter is decreased, so correlations are more readily preferred to starting new plan hypotheses. After this tuning, the separation between the two targets is improved from $33 \%$ to $17 \%$ (optimally $0 \%$ indicating an immediate correct separation) and the track continuity increases to $67 \%$.

The second littoral example concerns a coordinated attack of two Fast Patrol Boats on a Frigate that is moored before the coast. The initial plan recognition results are quite poor and in the best assignment hypothesis, there are four Fast Patrol Boats in stead of two. Also, observation of a jamming action is not incorporated in the correlation yet, therefore the plan recognizer cannot discern the target of the attack properly.

The correlation is improved by increasing the process noise and by incorporating the observed action in the correlation. To improve the correlation, also the process noise is increased, allowing for larger correlation windows. Again, the new target density parameter is decreased, thus favoring correlations above new target plan hypotheses. As a result of the tuning, the plan recognizer achieves an immediate separation between the two patrol boats and an almost correct set of plan hypotheses and a $60 \%$ track contimuity.

In the evaluation of the performance, the following statements can be made:

1. it is not really possible to recognize complete plans, but only complete parts of a plan. It concerns the parts that are currently being executed or are in the near future. It is a design decision whether to apply complete 
plan hypotheses.

2. The Multiple Hypothesis Tracking model is a good model, but also a limited model given the complexity of our problem. One has to work with an initial set of parameters of the Multiple Hypothesis Tracking algorithm, and try to improve the performance by tuning these parameters. Then good performances can be achieved.

3. It is necessary to retain a significant number of assignment hypotheses after pruning. This is necessary in order to allow for sufficient assignment hypotheses, so that all objects occur as a new target in these hypotheses. Also an unlikely assignment hypotheses can become the optimal one after a few successful correlations with new observations.

4. The best hypothesis is used for the performance measures, but the operator of an automatic plan recognition system must be able to examine alternative assignment hypotheses to get a good impression of the tactical situation.

The work that is presented in this thesis are the first steps of research on the Multiple Hypothesis Tracking approach to Tactical Plan Recognition. Topics for future research is the application of $\mathrm{k}$-best assignment algorithms from the field of Operations Research. Also the type of plan hypotheses is restricted to non-hierarchical, single agent plans. However, military plans and organizations often are hierarchically organized and hierarchical plans are found here. As mentioned before, in this research we utilize complete plan hypotheses, but it is not possible to make certain statements about future plan parts. It is interesting to work with partial plan hypotheses here, only elaborating plan parts when they are near to execution. Also, the framework here must be extended to handie uncertain observations. In the military a confidence is always attached to human intelligence. Finally, the set of scenarios has to be increased. A division between a learning set, to tune the algorithms, and a test set, to test the performance of the tuning, of scenarios must be made. 


\section{Samenvatting}

Plan herkenning kan grofweg worden beschreven als het probleem van het vinden van plannen welke de basis zijn van menselijk gedrag. In het geval van Tactische Plan Herkenning wil men de een tegenstander zijn militaire plan herkennen. Informatie hierover komt van 'intelligence' en sensoren. Deze produceren observaties van de objecten en 'agents' van tegenstanders. Een abservatie bevat informatie over de huidige positie van een tegenstander zijn object, zijn snelheid en richting en, mogelijk, zijn acties. Met de observaties kan alleen een incompleet beeld van de militaire wereld worden gecreëerd; men heeft dan alleen een beperkte kennis van de tegenstander!. Vanwege de beperkte kennis van de militaire wereld, kan een plan herkenner alleen een aantal redelijke plan hypotheses genereren.

Traditioneel is plan herkenning bestudeerd, geformaliseerd en geïmplementeerd in gebieden als 'story understanding' en 'user modelling'. Dit proefschrift focusseert op militaire toepassingen, waar de taals van plan herkenning cruciaal is. Echter, de taak is relevant voor alle toepassingen welke omgaan met tegenstanders.

Tactische Plan Herkenning verschilt van traditionele plan herkenning in een aantal manieren. Een tegenstander zal altijd proberen zijn plannen geheim te houden. De doelstellingen van een vijandelijk plan worden soms alleen ten dele gehaald. Op een bepaald moment zijn er meerdere onafhankelijke of afhankelijke plan en een plan herkenner wordt geconfronteerd met een multitarget multi-sensor data fusie probleem. Dit werk focusseert op een bijzonder gevolg van het verbergen van gegevens tijdens een militaire operatie: namelijk dat het over het algemeen niet bekend is welke observaties van tegenstanders eenzelfde object of agent betreffen. De observaties zijn ongeidentificieerd. Ook kan de bewegingstoestand van een object of agent alleen geschat worden omdat de metingen van pasitie en snelheid van een object onzeker zijn, en in het algemeen gemodelleerd worden door stochastische variabelen. 
Andere systemen voor dit probleem bestaan uit een sequentile observatie correlator gevolgd door een plan herkenningsfunctie. De correlator verbind verschillende ongeïdentificeerde observaties met elkaar, welke gedacht worden dezelfde agent te betreffen. Op deze wijze worden de geïdentificeerde observaties gemaakt, welke dan invoer zijn van een traditionele plan herkenner voor geidentificeerde observaties. Nadelen van deze aanpak zijn: 1) niet alle informatie, welke beschikbaar is in een plan hypothese word daadwerkelijk gebruikt voor correlatie, en 2) niet alle mogelijke combinaties van observaties worden beschouwd door een correlator. Daarom wordt een directe behandeling van ongeidentificeerde observaties geprefereerd.

Tijdens de ontwikkeling van algoritmes, is het voordelig uitspraken over hun correctheid te doen. M.a.w. men wil de correctheid en compleetheid van het algoritme bespreken. Hiervoor is een formele beschrijving van Tactische Plan Herkenning nodig. Echter deze wordt niet echt gegeven in ander werk.

Dit werk behandelt de volgende onderzoeksvraag:

Kan een correct, compleet, optimaal and maakbaar ('feasible') software systeem worden ontwikkeld voor het Tactische Plan Herkenningsprobleem, welke ongeïdentificeerde observaties direct kan behandelen?

De formalisatie van Tactische Plan Herkenning, wellke hier wordt gepresenteerd, is gebaseerd op klassieke abductie. De concepten van klassieke abductie kunnen eenvoudig toegepast worden op plan herkenners voor geindentificeerde observaties. Echter, voor Tactische Planherkenning moeten hierop enkele aanpassingen worden gedaan. Hier zal de plan herkenner niet alleen plan hypotheses genereren, echter ook toekennings (assignment) hypotheses. Deze toekenningshypotheses leggen een relatie tussen observaties en hypothetische plannen. Een keuze voor toekenningshypotheses is essentieel voor een beslissing over de vraag welke observaties van eenzelfde object stammen. Hiermee is een formele beschrijving van Tactische Plan Herkeming beschikbaar en is eenvoudig toepasbaar voor definities betreffende correctheid en compleetheid van de algoritmen.

Voor observaties met stochastische variabelen, word de kans van een toekenningshypothese berekend, dit in plaats van de kans voor een plan hypothese zelf. Deze laatste methode wordt veel gevonden in traditionele Tactische Plan Herkenners. Voor de berekening van de kans van een toekenningshypothese kan Reid zijn multiple target tracking formule aangepast worden. 
De formele definities en de toepassing van Reid's formule zijn gellustreerd met een groot aantal voorbeelden van tactische situaties. Ze betreffen militaire scenarios en een civiele toepassing. De voorbeelden beginnen met het simpele geval van geïdentificeerde observaties tot de meer moeilijke ongeïdentificeerde observaties. In het laatste voorbeeld vindt men ongeidentificeerde observaties met stochastische variabelen voor bewegingsmetingen.

De formele definities geven een zeer preciese beschrijving van de invoer van een Tactische Plan Herkemer en relateren deze invoer met de vitvoer. De volgende fase in de ontwikkeling van een algoritme bestaat uit de analyse van het Tactische Planherkenningsprobleem. Dit wordt gedaan door een Generiek Taakmodel voor het probleem voor te stellen. Een Generiek Taakmodel beschrijft een klasse van problemen, dit eerder dan specifieke instantiaties van het probleem. Natuurlijk, in ons geval betreft het Tactische Plan Herkenning. Instantiaties van deze klasse zijn specifieke systemen, bijv. de Data Fusion Demonstrator welke ontwikkeld werd voor het leger domein. Twee Generieke 'Taakmodellen worden gepresenteerd: éen voor het eenvoudiger probleen met geidentificeerde observaties, gevolgd door én voor het onderhande probleem, Tactische Plan Herkenning met ongeïdentificeerde observaties.

Als de Generieke Taakmodellen beschikbaar zijn, kunnen algoritmes worden ontwikkeld. In dit proefschrift worden algoritmes gespecificeerd in zuiver functionele Haskell programeertaal. Eerst worden geschikte algoritmes voor de probleem herkemning en planning functies besproken. Dan wordt een geschilkt model voor de schatting van bewegingsvariabelen van object gekozen. Het blijkt dat een simpel model met een rechte uniforme beweging een veel betere schatting van de snelheid geeft dan het 'reverting' model van Mevassvik. Daarna worden complete plan herkenningsalgoritmen gegeven. Gelijk als in de vorige (analyse) fase, beginnen we met het simpele geval met geidentificeerde observaties. Hiervoor wordt een 'Generate-and-test' algoritme gepresenteerd. Dan word zijn correctheid en compleetheid besproken. Ook wordt de complexiteit behandeld.

Het eerste algoritme is nog vrij eenvoudig, maar het wordt gevolgd door het, nogal gecompliceerde, ongeidentificeerde observaties probleem. Het heeft enkele delen welke reeds in het geidentificeerde observatie algoritme voorkwamen. De discussie over de correctheid en compleetheid van het tweede algoritme is gebaseerd op de conclusies van het eerste algoritme. Voor wat betreft de complexiteit van het ongeidentificeerde observaties algoritme, is de focus op de geheugen complexiteit. Een beschrijving van het aantal toeken- 
ningshypotheses wordt gegeven als een functie van het aantal observaties, welke ingevoerd zijn. De complexiteit is bijzonder groot; in feite is het NietPolynominaal. Het gevolg is dat hypothese management nodig is ('pruning', data partitionering) en slechts problemen met een beperkte omvang behandeld kunnen worden.

Het laatste deel van het proefschrift betreft een evaluatie van de algoritmes. Dit wordt gedaan met Test en Evaluatie software. Het bestaat uit een Java Simulator en Display programma en de, eerder beschreven, Haskell implementatie van een Tactische Plan Herkenner. Zij communiceren middels XML en ASCII data bestanden. Twee scenarios worden gebruikt voor evaluatie: het eerste is het leger voorbeeld (welke al eerder gepresenteerd was), het tweede is een kust ('littoral') scenario.

Het leger voorbeeld wordt in detail bestudeerd. Het voorbeeld betreft twee aanvalshelicopters welke verschillende vijandelijke eenheden aanvallen. De toekenningshypotheses worden bestudeerd nadat een observatie verwerkt is door het algoritme. Hun kansen en de beste assignment hypothese worden bediscussieerd. De performance is niet erg goed: de twee doelen worden niet altijd herkend als twee gescheiden doelen en de continuteit van de plan hypotheses van het doel is minimaal $33 \%$.

Om de performance te verbeteren, worden enkele parameters 'getuned' voor het scenario. Deze praktijk van 'tuning' vindt men altijd in toepassingen waar modellen van de buitenwereld beperkt in detail zijn. De kwaliteit van de verzameling van correlaties tussen plan hypotheses en observaties is verbeterd door de correlatie "windows' te verkleinen. De 'new target density' parameter wordt vermindert, zodat observaties sneller nieuwe plan hypotheses opstarten. Na cleze 'tuning', is de scheiding tussen de twee doelen verbeterd van na $33 \%$ van de observaties tot reeds na $17 \%$ van de observaties. De track continuteit is verbeterd tot $67 \%$.

Het tweede kust scenario behandelt een gecordineerde aanval van twee 'Fast Patrol Boats' op een fregat, welke voor de kust is geankerd. De initiële performance van de plan herkenning is nogal slecht en in de beste toekenningshypothese zijn vier 'Fast Patrol Boats' i.p.v. twee. Tevens is een observatie van een storingsactie niet goed behandeld, waardoor de plan herkenner het doel van de aanval niet goed kan herkennen.

Het correlatie mechanisme is verbeterd door vergroting van de proces ruis parameter, waardoor correlatie 'windows' vergroot worden. Ook wordt weer de 'new target density' parameter vermindert, waardoor correlaties de voorkeur 
krijgen boven new target hypotheses. Ten gevolge van de tuning bereikt de plan herkenner een onmiddellijke scheiding van de twee boten en $60 \%$ contimuiteit.

In de evaluatie van de performance worden de volgende uitspraken gedaan:

1. het is niet goed mogelijke complete plannen te herkennen, maar slechts complete delen van een plan. Het betreft delen welke geëxecuteerd worden of welke in de nabije toekomst liggen. Het is een ontwerpbeslissing om complete plan hypotheses te gebruiken.

2. Het Multiple Hypothesis Tracking model is een goed model, maar ook een beperkt model voor ons probleem. Als een gevolg hiervan moet met d.m.v. tuning de performance van de plan herkenner verbeteren.

3. Het is nodig om een significant aantal toekenningshypotheses te bewaren na pruning. Dit is noodzakelijk om tijdens het opstarten voor iedere observatie een aparte plan hypothese bij te houden. Tevens is gebleken dat, in het begin, onwaarschijnlijke assignment hypotheses, in een later stadium (na behandeling van meer observaties) de beste kunnen worden.

4. De beste hypothese wordt gebruikt voor performance maten, maar de operator van een automatisch plan herkenningsprogramma moet ook alternatieve assignment hypotheses kunnen bestuderen om een goede indruk van alle redelijke hypotheses te krijgen.

Het, hier gepresenteerde werk, bevatten de eerste stappen op het gebied van de Multiple Target Tracking aanpak voor Tactische Plan Herkenning. Mogelijke onderwerpen voor toekomstig onderzoek zijn: de toepassing van $k$-beste toekenningsalgoritmen van het veld van Operations Research. Ook zijn nu de plan hypotheses beperkt tot niet hiërarchische welke slechts door één agent worden geëxecuteerd. Echter, militaire plannen en organisaties zijn vaak hiërarchisch georganiseerd en hiërarchische plannen vindt men hier. Zoals al eerder vermeld, werkten we in dit onderzoek met complete plan hypotheses. Het is interessant om met partile plan hypotheses te werken. Ook moet het raamwerk uitgebreid worden zodat onzekere observaties behandeld kumnen worden. In de militaire wereld is altijd een mate van vertrouwen (confdence) verbonden aan intelligence rapporten. Ten slotte, betreffende de tuning moeten 'leerverzamelingen' worden gebruikt, voor tuning, waar de kwaliteit dan wordt bepaald door 'testverzamelingen'. 


\section{Curriculum Vitea}

Frank Mulder was born at Winterswijk, the Netherlands at 12 august 1959. He went to high school in Hengelo. In 1983 he received a Batchelor's degree in Electrical Engineering from the technical college at Zwolle. In 1988 he received a Master's degree in Electrical Engineering, with honors, from the University of Twente at Enschede in the Netherlands.

Frank Mulder has since 1988 been employed by Thales Nederland B.V. He started his career at the research department of Thales. His research topic was the application of Artificial Intelligence techniques in civil and military Command and Control systems.

His application was an automated approach control system for an airfield; a topic from Air Traffic Control. This research was done in cooperation with Peter Braspenning of the University of Maastricht. 'Their cooperation resulted in a fully automated reactive planning system that automatically generated manoeuvre instructions for pilots. Here also a fully distributed blackboard system: SPLICE, developed by Thales Nederland B.V., was applied.

After the approach control project, followed work concerning planning of train traffic, generation of modes and controls for radars and other sensors and the modelling of military plans. Apart from the planning research, Frank Mulder also also done research in sensor data fusion. Here he developed a high performance bayesian classifier and more general forms of Reid's Multiple Hypothesis Tracking algorithm. Frank also was the first who adapted more the efficient Murty's k-best Multiple Hypothesis Tracking algorithm for application in multi target multi sensor tracking. Frank was a participant in some international research projects on sensor data fusion: the Data Fusion Demonstrator project and the TA10 project.

The most recent research of Frank Muider is again in a military setting and concerns automatic recognition of an enemy's tactical plans by means of sensor and human intelligence observations. Here results from planning research and 
sensor data fusion research are combined in an Multiple Hypothesis Tracking approach to Tactical Plan Recognition.

Frank Mulder"s research resulted into several scientific papers. His work was presented in some national and international conferences on Artificial Intelligence and Computer Science. For this he received two national best paper awards. He authored (with Frans Voorbraak as co-author) also an article on tactical plan recognition in the Information Fusion Journal. 